Article

\title{
Ab-Initio Calculations of Level Energies, Oscillator Strengths and Radiative Rates for E1 Transitions in Beryllium-Like Iron
}

\author{
Ahmed Abou El-Maaref ${ }^{1,2, *}$, Stefan Schippers ${ }^{2}$ and Alfred Müller ${ }^{2}$ \\ ${ }^{1}$ Physics Department, Faculty of Science, Al-Azhar University Assiut, 71524 Assiut, Egypt \\ 2 Institut für Atom- und Molekülphysik, Justus-Liebig-Universität Giessen, D-35392 Giessen, \\ Germany; E-Mails: Stefan.E.Schippers@iamp.physik.uni-giessen.de (S.S.); \\ Alfred.Mueller@iamp.physik.uni-giessen.de (A.M.) \\ * Author to whom correspondence should be addressed; E-Mail: aahmh@ hotmail.com; \\ Tel.: +20-1018918939.
}

Academic Editor: James F. Babb

Received: 10 November 2014 / Accepted: 9 January 2015 / Published: 20 January 2015

\begin{abstract}
In the present work, energy levels, oscillator strengths, radiative rates and wavelengths of Be-like iron $\left(\mathrm{Fe}^{22+}\right)$ from $a b$-initio calculations using the multiconfiguration Dirac-Hartree-Fock method are presented. These quantities have been calculated for a set of configurations in the general form $1 s^{2} n l n^{\prime} l^{\prime}$, where $n=2,3$ and $n^{\prime}=2,3,4,5$ and $l=s, p, d$ and $l^{\prime}=s, p, d, f, g$. In addition, excitations of up to four electrons, including core-electron excitations, have been considered to improve the quality of the wave functions. This study comprises an extensive set of E1 transition rates between states with different $J$. The present results are compared with the available experimental and theoretical data.
\end{abstract}

Keywords: energy levels; oscillator strengths; radiative rates; be-like iron

\section{Introduction}

Accurate atomic data for iron ions are of interest in astrophysics, especially for the identification of solar spectra [1-4], as well as in the physics of controlled fusion [5] and plasma diagnostic [6]. From the astrophysical point of view, the importance of iron ions lies in the fact that iron is the cosmically most abundant heavy element beyond silicon [7]. The beryllium isoelectronic sequence including the $\mathrm{Fe}^{22+}$ ion has been studied using different theoretical approaches [8-21]. Most of the earlier calculations have 
produced results for one- or two-electron excitations to low-lying levels (up to $n=2$ or 3). Excitation to high-lying levels was studied by Moribayashi and Kato [22], including configurations up to $2 p n l$ $(n \leq 20, l=s, p, d)$ using Cowan's code [23].

Recently, many theoretical calculations have been carried out for $\mathrm{Fe}^{22+}$ to meet the needs for accurate atomic structure data. Chidichimo et al. have calculated level energies for $n \leq 4$, as well as wavelengths and weighted oscillator strengths using the Belfast R-matrix programs [24,25]. Del Zanna et al. [26] have compiled experimental observations of energy levels and wavelengths, and performed calculations of weighted oscillator strengths for Be-like iron using the non-relativistic SUPERSTRUCTURE program. Santos et al. [27] calculated probabilities for transitions from the $1 s^{2} 2 s 3 p^{3} P_{0}$ level for selected beryllium-like ions, from $\mathrm{Z}=5$ to 92 . They used the MCDF method including relativistic effects, QED (quantum-electrodynamics) effects, and correlations up to the $4 f$ subshell, however, they neglected the Breit interaction. Jian-Hui et al. [28] have calculated energy levels, oscillator strengths, transition probabilities and wavelengths of $\mathrm{Fe}^{22+}$ using the MCDF method with the inclusion of vacuum polarization and Breit interaction. Charro et al. [29,30] have calculated some oscillator strengths of Be-like iron up to $n=2,3$ using the relativistic quantum defect orbital (RQDO) method and the MCDF method, but there were no Breit corrections included in the calculations. By using the FAC code Landi and Gu [31] calculated energy levels, oscillator strengths and transition probabilities for 166 fine-structure levels of $\mathrm{Fe}^{22+}$ belonging to the complexes $1 s^{2} 2 \ln l^{\prime}, n=2-5, l=s, p, d, f, g$. In the present work an extensive set of configuration state functions (CSF) including subshell populations up to the $5 g$ subshell is used. In addition, the Breit interaction and QED effects are incorporated, which have been neglected in most of the previous calculations. The present comprehensive treatment of the $\mathrm{Fe}^{22+}$ atomic structure aims at providing more accurate results than hitherto available.

For the present extensive atomic structure calculations of beryllium-like iron, $\mathrm{Fe}^{22+}$, we have used the multiconfiguration Dirac-Fock (MCDF) method [32] as implemented in the GRASP2K code [33]. Excitations from $n=3$ to $n=4,5$ (doubly-excited levels) are included, and EOL (extended optimal level) type calculations have been performed. Wavelengths, energy levels, and E1 transition parameters (oscillator strengths, transition probabilities, and line strengths) have been computed for 182 fine-structure levels. The calculations have been divided into two main groups, with even and odd parity. The odd-parity group contains 90 levels while the even-parity group has 92 levels. The present calculations of oscillator strengths and radiative rates are generally in a good agreement with corresponding values in the NIST atomic data compilation [34]. The good agreement between our length and velocity gauge values provides some indication (although not a sufficient one) for the accuracy of the wave functions used in the present study.

\section{Method of Calculation}

Details of the MCDF method as implemented in the GRASP2K code can be found in References [32,33]. For the nuclear charge distribution within the ${ }^{56} \mathrm{Fe}$ nucleus, we used the default Fermi distribution parameters suggested in GRASP2K. The initial estimate for the radial orbitals is generated by solving the Dirac equation in a Thomas-Fermi potential for a single reference configuration (i.e., the $2 s^{2}$ level for even levels and the $2 s 2 p$ level for odd levels) by allowing the single, double, 
triple, and quadruple excitations to active orbital sets with $n=2,3,4,5$. The self-consistent procedure (RSCF) including EOL type calculations (extended optimal levels) is done layer by layer, at each stage the outer orbitals are optimized. The EOL type calculations construct orbitals from an average energy functional in which the fine-structure levels are given the weight $(2 J+1)$ [30]. This procedure is performed for every $J$-value separately. The splitting of the atomic levels into different groups has been found to be a useful compromise between two basic requirements in the atomic calculations, first, to get accurate wave functions for the radiative-rate calculations, and second, to keep the procedures manageable even with a large number of CSFs [35]. The same computational method has been applied to the even parity levels. The RSCF calculations were followed by relativistic configuration interaction (RCI) calculations including the Breit interaction Hamiltonian [36]. For the Breit interaction, we used the default low-frequency-limit approximation of the first-order perturbation theory, as implemented in the GRASP2K code. The GRASP2K procedure JJ2LSJ was used for the transformation of ASFs (atomic state functions) from a jj-coupled CSF basis into a LSJ-coupled CSF basis [37].

The beryllium-like iron atomic system has four electrons. In the ground state two electrons reside in the closed K-shell and the other two in the closed $2 s$ subshell. In the first step of building the ground state wave function of the beryllium-like ion, only interactions between the two outer shell electrons were considered. In a second step the interaction with the atomic core was additionally considered since it is very important for the calculation of the wave functions of the excited states. The ground level of the $\mathrm{Fe}^{22+}$ ion is the $1 s^{2} 2 s^{2}{ }^{1} S_{0}$ level, and the excited levels under consideration in this work belong to $1 s^{2} n l n^{\prime} l^{\prime}$ configurations, where $n=2,3$ and $n^{\prime}=2,3,4,5$ and $l=s, p, d$ and $l^{\prime}=s, p, d, f, g$ with different angular momenta and parities. The open K-shell states are already included in our calculations as admixing correlations, but not explicitly provided. We categorized these levels into groups having the same angular momentum and parity. For example the even parity states with $J=3$ are represented by $57161 j j$-coupled CSF. As shown in Table 1, the wave function expansions increase rapidly in size by increasing $n l$ which means that we can get unpractically high numbers of CSFs for $n>5$. The numbers of CSFs which are generated by quadruple excitations are shown in Table 1 which illustrates the degree of complexity of the present calculations.

Table 1. Number of configuration state functions (CSFs) used in the atomic state function expansion for the given angular momentum and parity $\left(J^{P}\right)$ considering only quadruple excitations.

\begin{tabular}{cccccccc}
\hline $\boldsymbol{J}^{+}$ & $\mathbf{3 l}$ & $\mathbf{4 l}$ & $\mathbf{5 l}$ & $\boldsymbol{J}^{-}$ & $\mathbf{3 l}$ & $\mathbf{4 l}$ & $\mathbf{5 l}$ \\
\hline 0 & 211 & 2149 & 13,592 & 0 & 180 & 2040 & 13,302 \\
1 & 436 & 5384 & 36,634 & 1 & 460 & 5476 & 36,894 \\
2 & 534 & 7250 & 52,481 & 2 & 516 & 7168 & 52,238 \\
3 & 380 & 6930 & 57,161 & 3 & 392 & 6988 & 57,354 \\
4 & 228 & 5588 & 53,512 & 4 & 222 & 5540 & 53,342 \\
5 & 89 & 3650 & 43,358 & 5 & 90 & 3672 & 43,466 \\
\hline
\end{tabular}

\section{Results and Discussion}

The calculated total energies (in a.u.) and energy levels (in $\mathrm{eV}$ ) are shown in ascending order in Table 2 where also comparisons with literature values [26,34,38,39] are included. Our calculated level 
energies for $\mathrm{Fe}^{22+}$ are in good agreement with the NIST levels [34]. The relative deviation is generally better than $1.0 \%$, except for the levels (2); $1 s^{2} 2 s 2 p\left({ }^{3} P_{0}\right)$ and (3); $1 s^{2} 2 s 2 p\left({ }^{3} P_{1}\right)$, where the relative deviations from NIST energies are $1.16 \%$ and $1.21 \%$, respectively. These deviations may be due to the limited numbers of CSFs in the calculations. However, including more correlations by adding $6 l$ orbitals to the expansion would produce a number of CSFs greater than our computer memory can tolerate. As an illustration, the number of CSFs generated by quadruple (Q) excitation to $6 l$ orbitals with $J^{P}=2^{+}$ is 260,702 . The energy of level $33,1 s^{2} 2 p 3 d\left({ }^{1} D_{2}\right)$, differs from the corresponding NIST [34] value by $0.13 \%$, while the values by Del Zanna et al. [26] and the CHIANTI database [38] deviate from the NIST [34] value by $0.94 \%$ and $0.97 \%$, respectively.

Table 2. Total energies $E_{\text {total }}$ (in a.u.) and energy levels (in $\mathrm{eV}$ ) of Be-like iron $\left(\mathrm{Fe}^{22+}\right)$. The sixth column lists the present energy levels, and the next 5-columns provide the energies from NIST database [34], the observed energies by Del Zanna et al. [26] and Gu et al. [39], and the calculated (Theor.) and observed (Exp.) values from the CHIANTI database [38].

\begin{tabular}{|c|c|c|c|c|c|c|c|c|c|c|}
\hline Key & Configuration & $J$ & Parity & $E_{\text {total }}$ & Present & NIST & Reference [26] & $\begin{array}{c}\text { Reference [38] } \\
\text { Theor. }\end{array}$ & $\begin{array}{c}\text { Reference [38] } \\
\text { Exp. }\end{array}$ & Reference [39] \\
\hline 1 & $1 s^{2} 2 s^{2}\left({ }^{1} S\right)$ & 0 & + & -812.1123386 & 0.00 & 0.00 & 0.00 & 0.00 & 0.00 & 0.00 \\
\hline 2 & $1 s^{2} 2 s 2 p\left({ }^{3} P\right)$ & 0 & - & -810.5074865 & 43.67 & 43.1688 & 43.16 & 42.86 & 43.13 & 43.219 \\
\hline 3 & $1 s^{2} 2 s 2 p\left({ }^{3} P\right)$ & 1 & - & -810.4056986 & 46.44 & 47.0055 & 47 & 46.83 & 46.99 & 47.046 \\
\hline 4 & $1 s^{2} 2 s 2 p\left({ }^{3} P\right)$ & 2 & - & -809.9784327 & 58.07 & 58.4933 & 58.49 & 58.16 & 58.51 & 58.538 \\
\hline 5 & $1 s^{2} 2 s 2 p\left({ }^{1} P\right)$ & 1 & - & -808.6849085 & 93.26 & 93.2869 & 93.28 & 93.78 & 93.31 & 93.308 \\
\hline 6 & $1 s^{2} 2 p^{2}\left({ }^{3} P\right)$ & 0 & + & -807.7638371 & 118.33 & 118.541 & 118.54 & 118.27 & 118.44 & 118.599 \\
\hline 7 & $1 s^{2} 2 p^{2}\left({ }^{3} P\right)$ & 1 & + & -807.4272328 & 127.49 & 127.357 & 127.35 & 126.86 & 127.26 & 127.412 \\
\hline 8 & $1 s^{2} 2 p^{2}\left({ }^{3} P\right)$ & 2 & + & -807.2353664 & 132.71 & 132.874 & 132.87 & 132.87 & 132.84 & 132.937 \\
\hline 9 & $1 s^{2} 2 p^{2}\left({ }^{1} D\right)$ & 2 & + & -806.6250012 & 149.31 & 149.302 & 149.3 & 149.41 & 149.42 & 149.341 \\
\hline 10 & $1 s^{2} 2 p^{2}\left({ }^{1} S\right)$ & 0 & + & -805.6167768 & 176.75 & 176.38 & 176.38 & 176.93 & 176.42 & 176.383 \\
\hline 11 & $1 s^{2} 2 s 3 s\left({ }^{3} S\right)$ & 1 & + & -771.5817273 & 1102.87 & 1102.72 & 1105.07 & 1105.84 & 1105.10 & \\
\hline 12 & $1 s^{2} 2 s 3 s\left({ }^{1} S\right)$ & 0 & + & -771.2425609 & 1112.1 & & 1112.77 & 1114.73 & 1112.80 & \\
\hline 13 & $1 s^{2} 2 s 3 p\left({ }^{3} P\right)$ & 1 & - & -770.8241423 & 1123.48 & 1125.28 & 1125.26 & 1126.46 & 1125.20 & \\
\hline 14 & $1 s^{2} 2 s 3 p\left({ }^{3} P\right)$ & 0 & - & -770.779389 & 1124.7 & & & 1126.47 & 1125.18 & \\
\hline 15 & $1 s^{2} 2 s 3 p\left({ }^{1} P\right)$ & 1 & - & -770.679708 & 1127.41 & 1129.12 & 1129.16 & 1130.22 & 1129.20 & \\
\hline 16 & $1 s^{2} 2 s 3 p\left({ }^{3} P\right)$ & 2 & - & -770.6540603 & 1128.11 & & 1129.72 & 1130.64 & 1129.69 & \\
\hline 17 & $1 s^{2} 2 s 3 d\left({ }^{3} D\right)$ & 1 & + & -770.2600664 & 1138.83 & 1140.53 & 1140.43 & 1141.79 & 1140.33 & \\
\hline 18 & $1 s^{2} 2 s 3 d\left({ }^{3} D\right)$ & 2 & + & -770.2535233 & 1139.01 & 1141.77 & 1140.63 & 1142.39 & 1140.96 & \\
\hline 19 & $1 s^{2} 2 s 3 d\left({ }^{3} D\right)$ & 3 & + & -770.1730742 & 1141.2 & 1142.14 & 1142.03 & 1143.35 & 1141.97 & \\
\hline 20 & $1 s^{2} 2 s 3 d\left({ }^{1} D\right)$ & 2 & + & -769.9289828 & 1147.84 & 1149.71 & 1149.62 & 1151.31 & 1149.65 & \\
\hline 21 & $1 s^{2} 2 p 3 s\left({ }^{3} P\right)$ & 0 & - & -769.5356786 & 1158.54 & 1152.43 & & 1160.33 & 1159.01 & \\
\hline 22 & $1 s^{2} 2 p 3 s\left({ }^{3} P\right)$ & 1 & - & -769.495422 & 1159.64 & & & 1162.56 & 1161.19 & \\
\hline 23 & $1 s^{2} 2 p 3 p\left({ }^{3} D\right)$ & 1 & + & -769.0671809 & 1171.29 & 1172.27 & & 1173.74 & 1172.67 & \\
\hline 24 & $1 s^{2} 2 p 3 s\left({ }^{3} P\right)$ & 2 & - & -769.0006032 & 1173.1 & & 1174.91 & 1175.29 & 1174.46 & \\
\hline 25 & $1 s^{2} 2 p 3 s\left({ }^{1} P\right)$ & 1 & - & -768.7864995 & 1178.93 & 1174.1 & 1180.15 & 1181.23 & 1180.19 & \\
\hline 26 & $1 s^{2} 2 p 3 p\left({ }^{3} D\right)$ & 2 & + & -768.7789536 & 1179.13 & 1180.83 & 1180.8 & 1181.79 & 1180.88 & \\
\hline 27 & $1 s^{2} 2 p 3 p\left({ }^{1} P\right)$ & 1 & + & -768.7562887 & 1179.75 & 1181.07 & & 1181.94 & 1181.03 & \\
\hline 28 & $1 s^{2} 2 p 3 p\left({ }^{3} P\right)$ & 0 & + & -768.5650136 & 1184.96 & & & 1184.87 & 1183.43 & \\
\hline 29 & $1 s^{2} 2 p 3 d\left({ }^{3} F\right)$ & 2 & - & -768.5068132 & 1186.54 & & & 1189.09 & 1188.16 & \\
\hline 30 & $1 s^{2} 2 p 3 p\left({ }^{3} P\right)$ & 1 & + & -768.3334484 & 1191.26 & & & 1192.9 & 1192.22 & \\
\hline 31 & $1 s^{2} 2 p 3 d\left({ }^{3} F\right)$ & 3 & - & -768.287093 & 1192.52 & 1193.35 & 1193.27 & 1194.45 & 1193.11 & \\
\hline 32 & $1 s^{2} 2 p 3 p\left({ }^{3} D\right)$ & 3 & + & -768.2775959 & 1192.78 & 1193.22 & 1193.12 & 1193.59 & 1193.56 & \\
\hline 33 & $1 s^{2} 2 p 3 d\left({ }^{1} D\right)$ & 2 & - & -768.2540191 & 1193.42 & 1194.96 & 1206.11 & 1206.61 & 1195.29 & \\
\hline 34 & $1 s^{2} 2 p 3 p\left({ }^{3} S\right)$ & 1 & + & -768.2240512 & 1194.23 & & & 1195.94 & 1195.17 & \\
\hline 35 & $1 s^{2} 2 p 3 p\left({ }^{3} P\right)$ & 2 & + & -768.218499 & 1194.38 & 1195.7 & 1195.64 & 1196.42 & 1195.61 & \\
\hline
\end{tabular}


Table 2. Cont.

\begin{tabular}{|c|c|c|c|c|c|c|c|c|c|c|}
\hline Key & Configuration & $J$ & Parity & $E_{\text {total }}$ & Present & NIST & Reference [26] & $\begin{array}{c}\text { Reference [38] } \\
\text { Theor. }\end{array}$ & $\begin{array}{l}\text { Reference [38] } \\
\text { Exp. }\end{array}$ & Reference [39] \\
\hline 36 & $1 s^{2} 2 p 3 d\left({ }^{3} D\right)$ & 1 & - & -768.1717335 & 1195.66 & 1194.84 & 1198.03 & 1198.49 & 1197.51 & \\
\hline 37 & $1 s^{2} 2 p 3 p\left({ }^{1} D\right)$ & 2 & + & -767.9295858 & 1202.25 & 1203.76 & 1203.61 & 1204.21 & 1203.62 & \\
\hline 38 & $1 s^{2} 2 p 3 d\left({ }^{3} D\right)$ & 2 & - & -767.8457058 & 1204.53 & 1206.12 & 1194.87 & 1196.01 & 1204.91 & \\
\hline 39 & $1 s^{2} 2 p 3 d\left({ }^{3} F\right)$ & 4 & - & -767.8033102 & 1205.68 & & 1205.09 & 1205.33 & 1206.13 & \\
\hline 40 & $1 s^{2} 2 p 3 d\left({ }^{3} D\right)$ & 3 & - & -767.7047396 & 1208.36 & 1208.72 & 1209.19 & 1209.8 & 1209.32 & \\
\hline 41 & $1 s^{2} 2 p 3 d\left({ }^{3} P\right)$ & 1 & - & -767.6426327 & 1210.05 & & & 1212.27 & 1211.77 & \\
\hline 42 & $1 s^{2} 2 p 3 d\left({ }^{3} P\right)$ & 2 & - & -767.641839 & 1210.08 & 1209.22 & 1211.63 & 1212.18 & 1211.80 & \\
\hline 43 & $1 s^{2} 2 p 3 d\left({ }^{3} P\right)$ & 0 & - & -767.5905258 & 1211.47 & & & 1212.55 & 1212.07 & \\
\hline 44 & $1 s^{2} 2 p 3 p\left({ }^{1} S\right)$ & 0 & + & -767.5265103 & 1213.21 & & & 1214.11 & 1213.37 & \\
\hline 45 & $1 s^{2} 2 p 3 d\left({ }^{1} F\right)$ & 3 & - & -767.3347315 & 1218.43 & 1218.8 & 1218.61 & 1220.05 & 1218.89 & \\
\hline 46 & $1 s^{2} 2 p 3 d\left({ }^{1} P\right)$ & 1 & - & -767.3208321 & 1218.81 & 1218.52 & & 1221.25 & 1220.56 & \\
\hline 47 & $1 s^{2} 2 s 4 s\left({ }^{3} S\right)$ & 1 & + & -757.6906561 & 1480.86 & & & 1484.44 & 1482.28 & \\
\hline 48 & $1 s^{2} 2 s 4 s\left({ }^{1} S\right)$ & 0 & + & -757.5602444 & 1484.4 & 1485.45 & 1485.4 & 1487.3 & 1485.43 & \\
\hline 49 & $1 s^{2} 2 s 4 p\left({ }^{3} P\right)$ & 1 & - & -757.3878963 & 1489.09 & 1490.79 & 1491.06 & 1492.46 & 1490.46 & \\
\hline 50 & $1 s^{2} 2 s 4 p\left({ }^{3} P\right)$ & 0 & - & -757.370068 & 1489.58 & & & 1492.24 & 1490.73 & \\
\hline 51 & $1 s^{2} 2 s 4 p\left({ }^{3} P\right)$ & 2 & - & -757.3205243 & 1490.93 & & & 1493.93 & 1492.42 & \\
\hline 52 & $1 s^{2} 2 s 4 p\left({ }^{1} P\right)$ & 1 & - & -757.3072762 & 1491.29 & 1493.27 & 1493.23 & 1494.55 & 1493.23 & \\
\hline 53 & $1 s^{2} 2 s 4 d\left({ }^{3} D\right)$ & 2 & + & -757.1611615 & 1495.26 & 1497.11 & 1497.07 & 1498.77 & 1497.02 & \\
\hline 54 & $1 s^{2} 2 s 4 d\left({ }^{3} D\right)$ & 1 & + & -757.1547887 & 1495.44 & 1496.86 & 1496.83 & 1498.57 & 1497.25 & \\
\hline 55 & $1 s^{2} 2 s 4 d\left({ }^{3} D\right)$ & 3 & + & -757.1038753 & 1496.82 & 1497.85 & 1497.79 & 1499.15 & 1497.68 & \\
\hline 56 & $1 s^{2} 2 s 4 d\left({ }^{1} D\right)$ & 2 & + & -757.051647 & 1498.24 & 1499.96 & 1499.93 & 1501.61 & 1499.97 & \\
\hline 57 & $1 s^{2} 2 s 4 f\left({ }^{3} F\right)$ & 2 & - & -757.029191 & 1498.85 & & & 1502.11 & 1500.45 & \\
\hline 58 & $1 s^{2} 2 s 4 f\left({ }^{3} F\right)$ & 3 & - & -757.0029963 & 1499.57 & & & 1502.21 & 1500.55 & \\
\hline 59 & $1 s^{2} 2 s 4 f\left({ }^{1} F\right)$ & 3 & - & -756.9746835 & 1500.34 & & & 1503.03 & 1500.75 & \\
\hline 60 & $1 s^{2} 2 s 4 f\left({ }^{3} F\right)$ & 4 & - & -756.9456257 & 1501.13 & & & 1502.39 & 1501.37 & \\
\hline 61 & $1 s^{2} 2 p 4 s\left({ }^{3} P\right)$ & 1 & - & -755.8087174 & 1532.06 & & & 1535.31 & 1532.53 & \\
\hline 62 & $1 s^{2} 2 p 4 s\left({ }^{3} P\right)$ & 0 & - & -755.8024205 & 1532.24 & & & 1534.72 & 1533.18 & \\
\hline 63 & $1 s^{2} 2 p 4 p\left({ }^{3} D\right)$ & 1 & + & -755.6064384 & 1537.57 & & & 1540.47 & 1538.88 & \\
\hline 64 & $1 s^{2} 2 p 4 p\left({ }^{3} D\right)$ & 2 & + & -755.482694 & 1540.94 & 1542.74 & & 1543.87 & 1542.36 & \\
\hline 65 & $1 s^{2} 2 p 4 p\left({ }^{3} P\right)$ & 1 & + & -755.4767887 & 1541.1 & & & 1543.64 & 1542.64 & \\
\hline 66 & $1 s^{2} 2 p 4 d\left({ }^{3} F\right)$ & 2 & - & -755.3757501 & 1543.85 & & & 1546.68 & 1542.87 & \\
\hline 67 & $1 s^{2} 2 p 4 d\left({ }^{3} P\right)$ & 2 & - & -755.3004331 & 1545.9 & & 1547.36 & 1548.6 & 1545.15 & \\
\hline 68 & $1 s^{2} 2 p 4 d\left({ }^{3} F\right)$ & 3 & - & -755.2586403 & 1547.03 & 1547.82 & 1547.7 & 1549.2 & 1547.27 & \\
\hline 69 & $1 s^{2} 2 p 4 d\left({ }^{3} D\right)$ & 1 & - & -755.2580034 & 1547.05 & 1548.31 & 1548.22 & 1549.88 & 1547.79 & \\
\hline 70 & $1 s^{2} 2 p 4 f\left({ }^{3} G\right)$ & 4 & + & -755.241964 & 1547.49 & & & 1551.14 & 1548.74 & \\
\hline 71 & $1 s^{2} 2 p 4 s\left({ }^{3} P\right)$ & 2 & - & -755.229847 & 1547.82 & & & 1550.3 & 1548.90 & \\
\hline 72 & $1 s^{2} 2 p 4 f\left({ }^{3} F\right)$ & 2 & + & -755.2296258 & 1547.82 & & & 1550.98 & 1549.08 & \\
\hline 73 & $1 s^{2} 2 p 4 f\left({ }^{3} G\right)$ & 3 & + & -755.2292762 & 1547.83 & & & 1550.57 & 1549.50 & \\
\hline 74 & $1 s^{2} 2 p 4 f\left({ }^{3} D\right)$ & 3 & + & -755.2093178 & 1548.37 & & & 1551.08 & 1549.62 & \\
\hline 75 & $1 s^{2} 2 p 4 s\left({ }^{1} P\right)$ & 1 & - & -755.2038121 & 1548.52 & & & 1551.12 & 1549.62 & \\
\hline 76 & $1 s^{2} 2 p 4 p\left({ }^{1} P\right)$ & 1 & + & -754.9800163 & 1554.61 & & & 1556.65 & 1549.66 & \\
\hline 77 & $1 s^{2} 2 p 4 p\left({ }^{3} P\right)$ & 2 & + & -754.9598815 & 1555.16 & & & 1560.57 & 1555.67 & \\
\hline 78 & $1 s^{2} 2 p 4 p\left({ }^{3} D\right)$ & 3 & + & -754.9258014 & 1556.09 & 1557.2 & 1557.12 & 1557.48 & 1556.78 & \\
\hline 79 & $1 s^{2} 2 p 4 p\left({ }^{3} S\right)$ & 1 & + & -754.925425 & 1556.1 & & & 1558.15 & 1556.62 & \\
\hline 80 & $1 s^{2} 2 p 4 p\left({ }^{1} D\right)$ & 2 & + & -754.8394761 & 1558.44 & & 1557.12 & 1557.52 & 1557.12 & \\
\hline 81 & $1 s^{2} 2 p 4 d\left({ }^{1} D\right)$ & 2 & - & -754.7748959 & 1560.2 & 1561.83 & 1561.79 & 1562.34 & 1559.90 & \\
\hline 82 & $1 s^{2} 2 p 4 p\left({ }^{1} S\right)$ & 0 & + & -754.7230568 & 1561.61 & & & 1564.05 & 1561.31 & \\
\hline 83 & $1 s^{2} 2 p 4 d\left({ }^{3} D\right)$ & 3 & - & -754.7134465 & 1561.87 & 1562.57 & 1562.54 & 1563.33 & 1561.67 & \\
\hline 84 & $1 s^{2} 2 p 4 d\left({ }^{3} P\right)$ & 1 & - & -754.7071109 & 1562.04 & 1564.06 & 1564.02 & 1564.28 & 1562.55 & \\
\hline 85 & $1 s^{2} 2 p 4 d\left({ }^{3} P\right)$ & 2 & - & -754.7010038 & 1562.21 & 1563.94 & 1563.9 & 1564.28 & 1563.46 & \\
\hline 86 & $1 s^{2} 2 p 4 d\left({ }^{3} F\right)$ & 4 & - & -754.6940971 & 1562.39 & & & 1562.34 & 1563.70 & \\
\hline 87 & $1 s^{2} 2 p 4 f\left({ }^{3} F\right)$ & 4 & + & -754.6817078 & 1562.73 & & & 1565.57 & 1563.66 & \\
\hline 88 & $1 s^{2} 2 p 4 d\left({ }^{3} P\right)$ & 0 & - & -754.6731993 & 1562.96 & & & 1564.42 & 1563.79 & \\
\hline 89 & $1 s^{2} 2 p 4 f\left({ }^{3} F\right)$ & 2 & + & -754.6511138 & 1563.56 & & & & & \\
\hline
\end{tabular}


Table 2. Cont.

\begin{tabular}{|c|c|c|c|c|c|c|c|c|c|c|}
\hline Key & Configuration & $J$ & Parity & $E_{t o t a l}$ & Present & NIST & Reference [26] & $\begin{array}{c}\text { Reference [38] } \\
\text { Theor. }\end{array}$ & $\begin{array}{l}\text { Reference [38] } \\
\text { Exp. }\end{array}$ & Reference [39] \\
\hline 90 & $1 s^{2} 2 p 4 f\left({ }^{1} G\right)$ & 4 & + & -754.6473417 & 1563.67 & & & 1566.63 & 1565.69 & \\
\hline 91 & $1 s^{2} 2 p 4 f\left({ }^{1} F\right)$ & 3 & + & -754.6449993 & 1563.73 & & & 1565.24 & 1564.31 & \\
\hline 92 & $1 s^{2} 2 p 4 f\left({ }^{3} D\right)$ & 3 & + & -754.6115814 & 1564.64 & & & & & \\
\hline 93 & $1 s^{2} 2 p 4 f\left({ }^{3} D\right)$ & 1 & + & -754.603951 & 1564.85 & & & 1566.91 & 1566.04 & \\
\hline 94 & $1 s^{2} 2 p 4 f\left({ }^{1} D\right)$ & 2 & + & -754.5999827 & 1564.95 & & & 1566.07 & 1565.16 & \\
\hline 95 & $1 s^{2} 2 p 4 d\left({ }^{1} P\right)$ & 1 & - & -754.5893505 & 1565.24 & & 1563.96 & 1567.36 & 1566.94 & \\
\hline 96 & $1 s^{2} 2 p 4 d\left({ }^{1} F\right)$ & 3 & - & -754.5841823 & 1565.38 & 1566.04 & 1565.88 & 1566.78 & 1566.04 & \\
\hline 97 & $1 s^{2} 2 s 5 s\left({ }^{3} S\right)$ & 1 & + & -751.392181 & 1652.24 & & & 1653.77 & & \\
\hline 98 & $1 s^{2} 2 s 5 s\left({ }^{1} S\right)$ & 0 & + & -751.3292255 & 1653.95 & & 1655.94 & 1655.09 & & \\
\hline 99 & $1 s^{2} 2 s 5 p\left({ }^{3} P\right)$ & 1 & - & -751.246659 & 1656.2 & & 1659.28 & 1657.83 & & \\
\hline 100 & $1 s^{2} 2 s 5 p\left({ }^{3} P\right)$ & 0 & - & -751.221321 & 1656.89 & & & 1657.73 & & \\
\hline 101 & $1 s^{2} 2 s 5 p\left({ }^{3} P\right)$ & 2 & - & -751.2099355 & 1657.2 & & & 1658.66 & & \\
\hline 102 & $1 s^{2} 2 s 5 p\left({ }^{1} P\right)$ & 1 & - & -751.2011308 & 1657.44 & 1659.28 & 1659.28 & 1659.03 & & \\
\hline 103 & $1 s^{2} 2 s 5 d\left({ }^{3} D\right)$ & 2 & + & -751.1374152 & 1659.17 & 1661 & 1661.34 & 1661.04 & & \\
\hline 104 & $1 s^{2} 2 s 5 d\left({ }^{3} D\right)$ & 1 & + & -751.126486 & 1659.47 & 1657.54 & & 1660.94 & & \\
\hline 105 & $1 s^{2} 2 s 5 d\left({ }^{1} D\right)$ & 2 & + & -751.0866158 & 1660.56 & 1666.1 & 1666.05 & 1662.22 & & \\
\hline 106 & $1 s^{2} 2 s 5 d\left({ }^{3} D\right)$ & 1 & + & -751.126486 & 1659.47 & & 1659.43 & 1660.94 & & \\
\hline 107 & $1 s^{2} 2 s 5 d\left({ }^{3} D\right)$ & 3 & + & -751.0860762 & 1660.57 & 1661.88 & 1661.77 & 1661.23 & & \\
\hline 108 & $1 s^{2} 2 s 5 g\left({ }^{3} G\right)$ & 4 & + & -751.0857587 & 1660.58 & & & 1662.95 & & \\
\hline 109 & $1 s^{2} 2 s 5 g\left({ }^{1} G\right)$ & 4 & + & -751.0826556 & 1660.66 & & & 1663.04 & & \\
\hline 110 & $1 s^{2} 2 s 5 f\left({ }^{3} F\right)$ & 2 & - & -751.0680262 & 1661.06 & & & 1662.78 & & \\
\hline 111 & $1 s^{2} 2 s 5 f\left({ }^{3} F\right)$ & 3 & - & -751.041488 & 1661.78 & & & 1662.81 & & \\
\hline 112 & $1 s^{2} 2 s 5 f\left({ }^{1} F\right)$ & 3 & - & -751.0238241 & 1662.26 & & & 1663.23 & & \\
\hline 113 & $1 s^{2} 2 s 5 g\left({ }^{3} G\right)$ & 3 & + & -751.0218288 & 1662.32 & & & 1662.95 & & \\
\hline 114 & $1 s^{2} 2 s 5 g\left({ }^{3} G\right)$ & 5 & + & -751.0212189 & 1662.34 & & & 1663.02 & & \\
\hline 115 & $1 s^{2} 2 s 5 f\left({ }^{3} F\right)$ & 4 & - & -750.9786648 & 1663.49 & & & 1662.9 & & \\
\hline 116 & $1 s^{2} 2 p 5 s\left({ }^{3} P\right)$ & 1 & - & -749.564771 & 1701.97 & & & 1703.76 & & \\
\hline 117 & $1 s^{2} 2 p 5 s\left({ }^{3} P\right)$ & 0 & - & -749.5389946 & 1702.67 & & & 1703.54 & & \\
\hline 118 & $1 s^{2} 2 p 5 p\left({ }^{3} D\right)$ & 1 & + & -749.4542569 & 1704.97 & & & 1706.64 & & \\
\hline 119 & $1 s^{2} 2 p 5 p\left({ }^{3} P\right)$ & 0 & + & -749.4006235 & 1706.43 & & & 1708.4 & & \\
\hline 120 & $1 s^{2} 2 p 5 p\left({ }^{3} D\right)$ & 2 & + & -749.3994227 & 1706.47 & & & 1708.39 & & \\
\hline 121 & $1 s^{2} 2 p 5 p\left({ }^{3} P\right)$ & 1 & + & -749.3905489 & 1706.71 & & & 1708.26 & & \\
\hline 122 & $1 s^{2} 2 p 5 d\left({ }^{3} F\right)$ & 2 & - & -749.3402395 & 1708.08 & & & 1709.85 & & \\
\hline 123 & $1 s^{2} 2 p 5 g\left({ }^{3} H\right)$ & 5 & - & -749.3026955 & 1709.1 & & & 1712.02 & & \\
\hline 124 & $1 s^{2} 2 p 5 d\left({ }^{3} P\right)$ & 2 & - & -749.2983472 & 1709.22 & & & 1710.88 & & \\
\hline 125 & $1 s^{2} 2 p 5 d\left({ }^{3} D\right)$ & 1 & - & -749.2867415 & 1709.53 & & & 1711.22 & & \\
\hline 126 & $1 s^{2} 2 p 5 f\left({ }^{3} G\right)$ & 4 & + & -749.2824452 & 1709.65 & & & 1712.13 & & \\
\hline 127 & $1 s^{2} 2 p 5 f\left({ }^{3} F\right)$ & 2 & + & -749.2733538 & 1709.9 & & & 1712.07 & & \\
\hline 128 & $1 s^{2} 2 p 5 f\left({ }^{3} G\right)$ & 3 & + & -749.2659755 & 1710.1 & & & 1711.84 & & \\
\hline 129 & $1 s^{2} 2 p 5 d\left({ }^{3} F\right)$ & 3 & - & -749.2653948 & 1710.11 & 1711.48 & & 1711.07 & & \\
\hline 130 & $1 s^{2} 2 p 5 f\left({ }^{3} D\right)$ & 3 & + & -749.2555687 & 1710.38 & & & 1712.11 & & \\
\hline 131 & $1 s^{2} 2 p 5 g\left({ }^{3} G\right)$ & 3 & - & -749.2352951 & 1710.93 & & & 1711.97 & & \\
\hline 132 & $1 s^{2} 2 p 5 g\left({ }^{3} H\right)$ & 4 & - & -749.1684219 & 1712.75 & & & 1711.93 & & \\
\hline 133 & $1 s^{2} 2 p 5 g\left({ }^{3} F\right)$ & 4 & - & -749.1647046 & 1712.85 & & & 1712.05 & & \\
\hline 134 & $1 s^{2} 2 p 5 s\left({ }^{3} P\right)$ & 2 & - & -748.9871642 & 1717.68 & & & 1719.42 & & \\
\hline 135 & $1 s^{2} 2 p 5 s\left({ }^{1} P\right)$ & 1 & - & -748.9732118 & 1718.06 & & & 1719.89 & & \\
\hline 136 & $1 s^{2} 2 p 5 p\left({ }^{3} D\right)$ & 2 & + & -748.8524328 & 1721.35 & & & & & \\
\hline 137 & $1 s^{2} 2 p 5 p\left({ }^{1} P\right)$ & 1 & + & -748.8511734 & 1721.38 & & & 1723.03 & & \\
\hline 138 & $1 s^{2} 2 p 5 p\left({ }^{3} S\right)$ & 1 & + & -748.8228875 & 1722.15 & & & 1723.76 & & \\
\hline 139 & $1 s^{2} 2 p 5 p\left({ }^{3} D\right)$ & 3 & + & -748.8108735 & 1722.48 & 1723.88 & & 1723.55 & & \\
\hline 140 & $1 s^{2} 2 p 5 p\left({ }^{1} D\right)$ & 2 & + & -748.7936606 & 1722.95 & & & 1723.32 & & \\
\hline 141 & $1 s^{2} 2 p 5 d\left({ }^{1} D\right)$ & 2 & - & -748.7499592 & 1724.14 & 1726.11 & & 1725.94 & & \\
\hline 142 & $1 s^{2} 2 p 5 p\left({ }^{1} S\right)$ & 0 & + & -748.7228871 & 1724.88 & & & 1726.27 & & \\
\hline
\end{tabular}


Table 2. Cont.

\begin{tabular}{|c|c|c|c|c|c|c|c|c|c|c|}
\hline Key & Configuration & $J$ & Parity & $E_{\text {total }}$ & Present & NIST & Reference [26] & $\begin{array}{c}\text { Reference [38] } \\
\text { Theor. }\end{array}$ & $\begin{array}{c}\text { Reference [38] } \\
\text { Exp. }\end{array}$ & Reference [39] \\
\hline 143 & $1 s^{2} 2 p 5 d\left({ }^{3} P\right)$ & 1 & - & -748.7220533 & 1724.9 & & & 1726.75 & & \\
\hline 144 & $1 s^{2} 2 p 5 d\left({ }^{3} P\right)$ & 2 & - & -748.7152748 & 1725.08 & & & & & \\
\hline 145 & $1 s^{2} 2 p 5 f\left({ }^{3} F\right)$ & 4 & + & -748.7106954 & 1725.21 & & & 1727.69 & & \\
\hline 146 & $1 s^{2} 2 p 5 d\left({ }^{3} D\right)$ & 3 & - & -748.7069321 & 1725.31 & 1726.98 & & 1726.34 & & \\
\hline 147 & $1 s^{2} 2 p 5 f\left({ }^{3} F\right)$ & 2 & + & -748.6949316 & 1725.64 & & & & & \\
\hline 148 & $1 s^{2} 2 p 5 f\left({ }^{1} G\right)$ & 4 & + & -748.6906284 & 1725.75 & & & 1728.16 & & \\
\hline 149 & $1 s^{2} 2 p 5 d\left({ }^{3} P\right)$ & 0 & - & -748.6802854 & 1726.03 & & & 1726.82 & & \\
\hline 150 & $1 s^{2} 2 p 5 f\left({ }^{1} F\right)$ & 3 & + & -748.6721365 & 1726.26 & & & 1727.54 & & \\
\hline 151 & $1 s^{2} 2 p 5 f\left({ }^{1} D\right)$ & 2 & + & -748.6702054 & 1726.31 & & & 1727.89 & & \\
\hline 152 & $1 s^{2} 2 p 5 d\left({ }^{1} P\right)$ & 1 & - & -748.6683712 & 1726.36 & & & 1728.05 & & \\
\hline 153 & $1 s^{2} 2 p 5 g\left({ }^{3} F\right)$ & 2 & - & -748.6669093 & 1726.4 & & & 1728.1 & & \\
\hline 154 & $1 s^{2} 2 p 5 f\left({ }^{3} D\right)$ & 1 & + & -748.6651871 & 1726.45 & & & 1728.25 & & \\
\hline 155 & $1 s^{2} 2 p 5 d\left({ }^{3} F\right)$ & 4 & - & -748.658763 & 1726.62 & & & 1725.99 & & \\
\hline 156 & $1 s^{2} 2 p 5 f\left({ }^{3} D\right)$ & 3 & + & -748.6564126 & 1726.68 & & & & & \\
\hline 157 & $1 s^{2} 2 p 5 g\left({ }^{3} G\right)$ & 3 & - & -748.6544227 & 1726.74 & & & & & \\
\hline 158 & $1 s^{2} 2 p 5 g\left({ }^{3} G\right)$ & 5 & - & -748.6511383 & 1726.83 & & & 1727.75 & & \\
\hline 159 & $1 s^{2} 2 p 5 d\left({ }^{1} F\right)$ & 3 & - & -748.6435973 & 1727.03 & 1728.96 & & 1727.74 & & \\
\hline 160 & $1 s^{2} 2 p 5 g\left({ }^{1} H\right)$ & 5 & - & -748.6425123 & 1727.06 & & & 1727.95 & & \\
\hline 161 & $1 s^{2} 2 p 5 g\left({ }^{3} F\right)$ & 3 & - & -748.63994 & 1727.13 & & & 1728.18 & & \\
\hline 162 & $1 s^{2} 2 p 5 f\left({ }^{3} G\right)$ & 5 & + & -748.6322597 & 1727.34 & & & 1727.98 & & \\
\hline 163 & $1 s^{2} 2 p 5 g\left({ }^{3} G\right)$ & 4 & - & -748.599593 & 1728.23 & & & 1727.87 & & \\
\hline 164 & $1 s^{2} 2 p 5 g\left({ }^{3} F\right)$ & 4 & - & -748.5922492 & 1728.43 & & & & & \\
\hline 165 & $1 s^{2} 2 p 4 p\left({ }^{3} P\right)$ & 0 & + & -746.0926537 & 1796.45 & & & & & \\
\hline 166 & $1 s^{2} 3 s^{2}\left({ }^{1} S\right)$ & 0 & + & -729.7643403 & 2240.75 & & & & & \\
\hline 167 & $1 s^{2} 3 s 3 p\left({ }^{3} P\right)$ & 0 & - & -729.3728951 & 2251.4 & & & & & \\
\hline 168 & $1 s^{2} 3 s 3 p\left({ }^{3} P\right)$ & 1 & - & -729.3646407 & 2251.63 & & & & & \\
\hline 169 & $1 s^{2} 3 s 3 p\left({ }^{3} P\right)$ & 2 & - & -729.2408485 & 2255 & & & & & \\
\hline 170 & $1 s^{2} 3 s 3 p\left({ }^{1} P\right)$ & 1 & - & -728.8209355 & 2266.42 & & & & & \\
\hline 171 & $1 s^{2} 3 s 3 d\left({ }^{1} D\right)$ & 2 & + & -728.7962086 & 2267.1 & & & & & \\
\hline 172 & $1 s^{2} 3 s 3 d\left({ }^{3} D\right)$ & 1 & + & -728.6774571 & 2270.33 & & & & & \\
\hline 173 & $1 s^{2} 3 s 3 d\left({ }^{3} D\right)$ & 2 & + & -728.667205 & 2270.61 & & & & & \\
\hline 174 & $1 s^{2} 3 s 3 d\left({ }^{3} D\right)$ & 3 & + & -728.604182 & 2272.32 & & & & & \\
\hline 175 & $1 s^{2} 3 p^{2}\left({ }^{3} P\right)$ & 0 & + & -728.5677089 & 2273.31 & & & & & \\
\hline 176 & $1 s^{2} 3 p^{2}\left({ }^{3} P\right)$ & 1 & + & -728.5185534 & 2274.65 & & & & & \\
\hline 177 & $1 s^{2} 3 p^{2}\left({ }^{3} P\right)$ & 2 & + & -728.4270966 & 2277.14 & & & & & \\
\hline 178 & $1 s^{2} 3 p 3 d\left({ }^{3} F\right)$ & 2 & - & -728.2811758 & 2281.11 & & & & & \\
\hline 179 & $1 s^{2} 3 p 3 d\left({ }^{3} F\right)$ & 3 & - & -728.1545448 & 2284.56 & & & & & \\
\hline 180 & $1 s^{2} 3 p 3 d\left({ }^{1} D\right)$ & 2 & - & -728.1238826 & 2285.39 & & & & & \\
\hline 181 & $1 s^{2} 3 p^{2}\left({ }^{1} D\right)$ & 2 & + & -728.0448765 & 2287.54 & & & & & \\
\hline 182 & $1 s^{2} 3 p^{2}\left({ }^{1} S\right)$ & 0 & + & -728.0435435 & 2287.58 & & & & & \\
\hline
\end{tabular}

One might wonder whether quadruple excitations are really necessary. Two examples illustrate the improvement of the results when quadruple excitations are included. The first example is level 14, $1 s^{2} 2 s 3 p\left({ }^{3} P_{0}\right)$, for which the present calculation gives an energy of $1124.70 \mathrm{eV}$ which agrees to within less than $0.2 \%$ with the value $1126.47 \mathrm{eV}$ recorded in the CHIANTI database [38]. If only triple excitations are included in the calculations the level energy drops to $1109.07 \mathrm{eV}$ with an increase of the deviation from the CHIANTI energy by as much as $1.5 \%$. The second example, that we want to mention, is level $21,1 s^{2} 2 p 3 s\left({ }^{3} P_{0}\right)$, which has a calculated energy of $1158.54 \mathrm{eV}$. This corresponds reasonably well to the NIST energy [34] $1152.40 \mathrm{eV}$ with a relative difference of less than $0.55 \%$. Again, when only triple excitations are considered, the level energy drops drastically, in this case to $1143.12 \mathrm{eV}$ producing a difference in the resulting level energy of $15.42 \mathrm{eV}$ and a relative deviation from the NIST energy 
of about $0.9 \%$. Obviously, the agreement of the calculations with the CHIANTI and NIST databases becomes considerably better with the use of 4-electron excitations.

Dirac-Fock wave functions with a minimum number of radial functions are not sufficient to represent the occupied orbitals. Extra configurations have to be added to adequately represent electron correlations (i.e., mixing coefficients). These extra configurations are represented by CSFs and must have the same angular momentum and parity as the occupied orbital [40]. For instance, the level $1 s^{2} 2 s 2 p\left({ }^{3} P_{0}^{-}\right)$is represented by 0.996 of $1 s^{2} 2 s 2 p\left({ }^{3} P_{0}^{-}\right)$and 0.0660 of $1 s^{2} 2 s 3 p\left({ }^{3} P_{0}^{-}\right)$. The mixing coefficients for the wave functions of some calculated levels are shown in Table 3. The most important contributions to the total wave function of a given level are those from the same configuration. For example, the configuration-mixed wave function for the $1 s^{2} 2 p 3 p\left({ }^{3} P_{0}\right)$ level is represented as

$$
\left|1 s^{2} 2 p 3 p\left({ }^{3} P_{0}\right)\right\rangle=0.9501\left|1 s^{2} 2 p 3 p\left({ }^{3} P_{0}\right)\right\rangle+0.3\left|1 s^{2} 2 p 3 p\left({ }^{1} S_{0}\right)\right\rangle-0.0697\left|1 s^{2} 2 s 3 s\left({ }^{1} S_{0}\right)\right\rangle
$$

where $0.9501,0.3$, and -0.0697 are the configuration mixing coefficients. Coefficients less than 0.05 were calculated but are not explicitly given. Expansion coefficients for several levels by Bhatia and Mason [10] are listed in Table 3 for comparison. Clearly, the present and the previous [10] results are very close to one another in the description of the configuration-interaction wave functions.

Table 3. The configuration mixing coefficients $(>0.05)$ for some levels in $\mathrm{Fe}^{22+}$. The number in the bra-kets refers to the level number (the key in Table 2).

\begin{tabular}{lll}
\hline$J^{P}$ & Present Work & Reference [10] \\
Configuration & & \\
\hline$J=0^{+}$ & & $0.98|1\rangle+0.21|10\rangle$ \\
$1 s^{2} 2 s^{2}\left({ }^{1} S\right)$ & $0.9772|1\rangle+0.2058|10\rangle$ & $0.97|6\rangle+0.24|10\rangle-0.07|1\rangle$ \\
$1 s^{2} 2 p^{2}\left({ }^{3} P\right)$ & $0.9663|6\rangle+0.2431|10\rangle-0.075|1\rangle$ & $0.95|10\rangle-0.25|6\rangle-0.19|1\rangle$ \\
$1 s^{2} 2 p^{2}\left({ }^{1} S\right)$ & $0.9465|10\rangle-0.2533|6\rangle-0.19241|1\rangle$ & $0.99|12\rangle+0.17|44\rangle$ \\
$1 s^{2} 2 s 3 s\left({ }^{1} S\right)$ & $0.9814|12\rangle+0.1688|44\rangle-0.0708|48\rangle$ & \\
$1 s^{2} 2 p 3 p\left({ }^{3} P\right)$ & $0.9501|28\rangle+0.3|44\rangle-0.0697|12\rangle$ & \\
\hline$J=1^{+}$ & & \\
$1 s^{2} 2 p^{2}\left({ }^{3} P\right)$ & $0.9992|7\rangle$ & $0.99|11\rangle+0.13|34\rangle$ \\
$1 s^{2} 2 s 3 s\left({ }^{3} S\right)$ & $0.9913|11\rangle+0.1301|34\rangle$ & $0.99|17\rangle+0.14|23\rangle$ \\
$1 s^{2} 2 s 3 d\left({ }^{3} D\right)$ & $0.9896|17\rangle+0.1399|23\rangle$ & \\
$1 s^{2} 2 p 3 p\left({ }^{3} D\right)$ & $0.8202|36\rangle+0.5328|27\rangle-0.1612|34\rangle+0.1265|17\rangle$ \\
$1 s^{2} 2 p 3 p\left({ }^{1} P\right)$ & $0.5302|27\rangle+0.5204|34\rangle+0.4903|30\rangle+0.4449|23\rangle$ & \\
\hline$J=2^{+}$ & & \\
$1 s^{2} 2 p^{2}\left({ }^{3} P\right)$ & $0.8640|8\rangle-0.5014|9\rangle$ & \\
$1 s^{2} 2 p^{2}\left({ }^{1} D\right)$ & $0.8637|9\rangle+0.5016|8\rangle$ & \\
$1 s^{2} 2 s 3 d\left({ }^{3} D\right)$ & $0.9903|18\rangle-0.1169|26\rangle$ & \\
$1 s^{2} 2 s 3 d\left({ }^{1} D\right)$ & $0.9895|20\rangle-0.1180|37\rangle$ & \\
$1 s^{2} 2 p 3 p\left({ }^{3} D\right)$ & $0.9169|26\rangle-0.2744|37\rangle+0.2595|35\rangle+0.1143|18\rangle$ & \\
\hline & & \\
& & \\
& & \\
& & \\
& & \\
\hline
\end{tabular}


Table 3. Cont.

\begin{tabular}{|c|c|c|}
\hline $\begin{array}{l}J^{P} \\
\text { Configuration }\end{array}$ & Present Work & Reference [10] \\
\hline \multicolumn{3}{|l|}{$J=3^{+}$} \\
\hline $1 s^{2} 2 s 3 d\left({ }^{3} D\right)$ & $0.9939|19\rangle-0.0943|32\rangle$ & \\
\hline $1 s^{2} 2 p 3 p\left({ }^{3} D\right)$ & $0.9948|32\rangle+0.0939|19\rangle$ & \\
\hline $1 s^{2} 2 s 4 d\left({ }^{3} D\right)$ & $0.9976|55\rangle$ & \\
\hline $1 s^{2} 2 p 4 f\left({ }^{3} G\right)$ & $0.8423|73\rangle+0.4515|91\rangle+0.2737|89\rangle$ & \\
\hline \multicolumn{3}{|l|}{$J=4^{+}$} \\
\hline $1 s^{2} 2 p 4 f\left({ }^{3} G\right)$ & $0.6431|70\rangle-0.5492|90\rangle+0.5300|87\rangle$ & \\
\hline $1 s^{2} 2 p 4 f\left({ }^{3} F\right)$ & $0.8133|87\rangle-0.5656|70\rangle+0.1220|90\rangle$ & \\
\hline $1 s^{2} 2 p 4 f\left({ }^{1} G\right)$ & $0.8241|90\rangle+0.5122|70\rangle+0.2327|87\rangle$ & \\
\hline $1 s^{2} 2 s 5 g\left({ }^{3} G\right)$ & $0.8292|108\rangle+0.5561|109\rangle$ & \\
\hline \multicolumn{3}{|l|}{$J=5^{+}$} \\
\hline $1 s^{2} 2 s 5 g\left({ }^{3} G\right)$ & $0.9982|114\rangle$ & \\
\hline $1 s^{2} 2 p 5 f\left({ }^{3} G\right)$ & $0.9980|162\rangle$ & \\
\hline \multicolumn{3}{|l|}{$J=0^{-}$} \\
\hline $1 s^{2} 2 s 2 p\left({ }^{3} P\right)$ & $0.9960|2\rangle+0.0660|14\rangle$ & $1.00|2\rangle$ \\
\hline $1 s^{2} 2 s 3 p\left({ }^{3} P\right)$ & $0.9908|14\rangle-0.0821|43\rangle$ & \\
\hline $1 s^{2} 2 p 3 s\left({ }^{3} P\right)$ & $0.9927|21\rangle+0.0714|43\rangle$ & \\
\hline $1 s^{2} 2 p 3 d\left({ }^{3} P\right)$ & $0.9918|43\rangle+0.0860|14\rangle$ & \\
\hline \multicolumn{3}{|l|}{$J=1^{-}$} \\
\hline $1 s^{2} 2 s 2 p\left({ }^{3} P\right)$ & $0.9840|3\rangle+0.1564|5\rangle$ & $0.99|3\rangle+0.16|5\rangle$ \\
\hline $1 s^{2} 2 s 2 p\left({ }^{1} P\right)$ & $0.9833|5\rangle-0.1568|3\rangle$ & $0.99|5\rangle-0.16|3\rangle$ \\
\hline $1 s^{2} 2 s 3 p\left({ }^{3} P\right)$ & $0.7772|13\rangle+0.5935|15\rangle-0.1644|25\rangle$ & $0.80|13\rangle+0.58|15\rangle-0.16|25\rangle$ \\
\hline $1 s^{2} 2 s 3 p\left({ }^{1} P\right)$ & $0.7411|15\rangle-0.6127|13\rangle-0.238|25\rangle$ & $0.76|15\rangle-0.59|13\rangle-0.24|25\rangle$ \\
\hline \multicolumn{3}{|l|}{$J=2^{-}$} \\
\hline $1 s^{2} 2 s 2 p\left({ }^{3} P\right)$ & $0.9965|4\rangle$ & \\
\hline $1 s^{2} 2 s 3 p\left({ }^{3} P\right)$ & $0.9890|16\rangle$ & \\
\hline $1 s^{2} 2 p 3 s\left({ }^{3} P\right)$ & $0.9843|24\rangle$ & \\
\hline $1 s^{2} 2 p 3 d\left({ }^{3} F\right)$ & $0.8727|29\rangle+0.4391|33\rangle+0.1375|38\rangle$ & \\
\hline \multicolumn{3}{|l|}{$J=3^{-}$} \\
\hline $1 s^{2} 2 p 3 d\left({ }^{3} F\right)$ & $0.8829|31\rangle+0.3724|40\rangle-0.2776|45\rangle$ & \\
\hline $1 s^{2} 2 p 3 d\left({ }^{3} D\right)$ & $0.8737|40\rangle-0.4342|31\rangle-0.2094|45\rangle$ & \\
\hline $1 s^{2} 2 p 3 d\left({ }^{1} F\right)$ & $0.9344|45\rangle+0.3064|40\rangle+0.1649|31\rangle$ & \\
\hline $1 s^{2} 2 s 4 f\left({ }^{3} F\right)$ & $0.9807|58\rangle+0.1863|59\rangle$ & \\
\hline \multicolumn{3}{|l|}{$J=4^{-}$} \\
\hline $1 s^{2} 23 d\left({ }^{3} F\right)$ & $0.9970|39\rangle$ & \\
\hline $1 s^{2} 2 s 4 f\left({ }^{3} F\right)$ & $0.9983|60\rangle$ & \\
\hline $1 s^{2} 2 p 4 d\left({ }^{3} F\right)$ & $0.9973|86\rangle$ & \\
\hline \multicolumn{3}{|l|}{$J=5^{-}$} \\
\hline $1 s^{2} 2 p 5 g\left({ }^{3} G\right)$ & $0.8469|158\rangle-0.4368\left|2 p 5 g\left({ }^{3} H\right)\right\rangle+0.2964|160\rangle$ & \\
\hline $1 s^{2} 3 d 4 f\left({ }^{3} H\right)$ & $0.9974|268\rangle$ & \\
\hline
\end{tabular}


A comparison between the calculated wavelengths and other published experimental and theoretical values $[1,2,8,24,26,28,29,34,41]$ is shown in Table 4 . The accuracy of calculated wavelengths (in $\AA$ ) relative to measurements [24,26,34] can be assessed from Table 4, where the agreement is within $<0.2 \%$ for all available transitions such as $1-5 ; 2 s^{2}\left({ }^{1} S_{0}\right)-2 s 2 p\left({ }^{1} P_{1}\right)$ with a calculated wavelength $\lambda=132.939 \AA$ which deviates from the measurements [24,26,34] by about $\pm 0.0248 \AA$ and from the calculated wavelength of Reference [42] by about $\pm 0.091 \AA$. In a few cases the agreement with measurements is slightly worse such as for the transitions 2-7, 3-6, 7-21, and 10-25 with $\lambda=147.922$, $172.469,12.025$, and $12.371 \AA$. In these cases the deviations from the measurements amount to $0.463 \%$, $0.485 \%, 0.579 \%$, and $0.451 \%$, respectively, which are much greater than the experimental uncertainties. The deviations actually reflect the estimated errors in the wavelengths. In Table 6 many more $\mathrm{Fe}^{22+}$ transitions in the soft X-ray region are listed than in any of the previous studies. This will help with the identification of spectral lines of the solar corona [1,2]. In particular, our calculations presented in Table 6 provide comprehensive new data for Be-like Fe with $\lambda$ in the range 8 to $17 \AA$ and at $132.85 \AA$ which are very important in the solar spectrum $[3,4]$.

The calculated values of transition probabilities in $\mathrm{s}^{-1}$, oscillator strengths $f$, and line strengths $S$ in a.u. (in both velocity and length forms) are listed in Table 6. A comparison between the present calculations of oscillator strengths $\left(f_{L}\right)$ and other published data $[8,24,26,29,34,41]$ is presented in Table 4. Most of our calculations of oscillator strengths show a good agreement with the NIST [34] values, but in a few cases the relative differences reach tens of \%. Actually, the NIST values are a compilation of experimental and theoretical data from previous works [8,41,43-47], and the estimated accuracies of most oscillator strengths and transition probabilities recorded in the NIST database [34] are quoted to be between $25 \%$ and $50 \%$. For transitions $1-5,2-7$, and 3-6 the uncertainty of the NIST data is estimated to be $\leq 10 \%$. The largest deviation in our calculations of oscillator strengths is found for the transition 3-23 where the deviation from NIST is 36\%. Generally, this error is still acceptable for oscillator strengths as is shown in previous publications, where the $f_{L}$ values for the transition 1-49 in References [24,26] differ from the corresponding NIST [34] value by 35\% and $33.25 \%$, respectively, and for the line 5-48 the difference is 20\% [26]. Our deviations for the same lines are about $6.75 \%, 12 \%$, respectively. The transition $1-5$ has an experimental $f_{L}$-value of 0.15 [48] with an estimated accuracy of $10 \%$. The agreement of our calculated value with this reference [48] is within $2 \%$. In the study by Jian-Hui et al. [28], significant differences compared with the NIST database [34] are revealed for several $f_{L}$ values, where the deviations of the transitions 5-23, 5-27, and 7-36 are more than one order of magnitude, while our calculations agree with NIST [34] within about $17 \%, 21.5 \%$ and $2.8 \%$, respectively. The precision of the theoretical calculations can be judged by the convergence between length and velocity gauge values of oscillator strengths. If exact wave functions are used then $f_{L}=f_{V}$ [49], which is convincingly fulfilled by the approximate wave functions that are used in our calculations. The average deviation between $f_{L}$ and $f_{V}$ values is about $30 \%$. There are several transitions with much larger deviations such as, the transitions 2-34, 2-47, 2-97, 3-48, 4-154, 11-116, 18-143, 93-149, 134-156, and 140-152. Comparisons between our transition probabilities and values from the NIST database [34] are available for some transitions. For instance, transitions 
$1-5,1-13$, and $1-15$ in Table 6 have $A_{L}$ values of $1.91 \mathrm{E}+10,4.99 \mathrm{E}+12$ and $7.94 \mathrm{E}+12 s^{-1}$ while the values from the NIST database for these transitions are $2.0 \mathrm{E}+10,4.9 \mathrm{E}+12$ and $7.9 \mathrm{E}+12 s^{-1}$, respectively. A comparison between the present GRASP2K calculations of transition probabilities and other published data $[28,41,42,50,51]$ is shown in Table 5 .

Table 4. Comparison between the present calculations of oscillator strengths $f_{L}$ (calc) and wavelengths ( $\lambda_{\text {calc }}$ in $\AA$ ) and other published data for some transitions in Be-like iron. LL. = lower level; UL. $=$ upper level, $\lambda_{N I S T}$ denotes the Ritz wavelength from the NIST database spectra.

\begin{tabular}{|c|c|c|c|c|c|c|c|c|}
\hline LL. & UL. & $f_{L}($ calc $)$ & $f_{L}$ Reference $[34,38]$ & Other $f_{L}$ Values & $\lambda_{\text {calc }}$ & Other Values of $\lambda$ & $\lambda_{o b s}$ & $\lambda_{N I S T}$ \\
\hline 1 & 5 & $1.52 \mathrm{E}-01$ & $1.55 \mathrm{E}-01^{1}$ & $1.50 \mathrm{E}-01^{a}, 1.55 \mathrm{E}-01^{b}, 1.54 \mathrm{E}-01^{c}$ & 132.939 & $132.906^{a}, 132.830^{d}$ & 132.906 & 132.910 \\
\hline 1 & 13 & $2.73 \mathrm{E}-01$ & $2.70 \mathrm{E}-01^{1}$ & $2.50 \mathrm{E}-01^{a}, 2.50 \mathrm{E}-01^{b}, 2.54 \mathrm{E}-01^{c}$ & 11.036 & $11.0063^{a}, 11.018^{b}, 11.017^{c}$ & 11.018 & 11.020 \\
\hline 1 & 15 & $4.32 \mathrm{E}-01$ & $4.30 \mathrm{E}-01^{1}$ & $4.10 \mathrm{E}-01^{a}, 4.10 \mathrm{E}-01^{b}, 4.14 \mathrm{E}-01^{c}$ & 10.997 & $10.9697^{a}, 10.980^{b}, 10.980^{c}$ & 10.980 & 10.980 \\
\hline 1 & 22 & $9.82 \mathrm{E}-03$ & $1.27 \mathrm{E}-02^{2}$ & $1.30 \mathrm{E}-02^{b}, 2.11 \mathrm{E}-02^{c}$ & 10.691 & $10.664^{2}, 10.6645^{b}, 10.505^{c}$ & & \\
\hline 1 & 36 & $5.97 \mathrm{E}-03$ & $5.92 \mathrm{E}-03^{2}$ & $5.00 \mathrm{E}-03^{b}, 5.91 \mathrm{E}-03^{c}$ & 10.369 & $10.349^{2}, 10.3448^{b}, 10.349^{c}$ & & 10.377 \\
\hline 1 & 41 & $2.41 \mathrm{E}-04$ & $2.32 \mathrm{E}-04^{2}$ & $2.00 \mathrm{E}-04^{b}, 2.32 \mathrm{E}-04^{c}$ & 10.246 & $10.233^{2}, 10.2272^{b}, 10.227^{c}$ & & \\
\hline 1 & 46 & $1.94 \mathrm{E}-02$ & $1.90 \mathrm{E}-02^{2}$ & $1.70 \mathrm{E}-02^{b}, 1.89 \mathrm{E}-02^{c}$ & 10.172 & $10.152^{2}, 10.152^{b}, 10.153^{c}$ & & 10.175 \\
\hline 1 & 49 & $3.73 \mathrm{E}-02$ & $4.00 \mathrm{E}-02^{1}$ & $2.60 \mathrm{E}-02^{a}, 2.60 \mathrm{E}-02^{b}, 3.60 \mathrm{E}-02^{d}$ & 8.326 & $8.3072^{a}, 8.315^{b}, 8.316^{d}$ & 8.317 & 8.320 \\
\hline 1 & 52 & $1.55 \mathrm{E}-01$ & $1.50 \mathrm{E}-01^{1}$ & $1.40 \mathrm{E}-01^{a}, 1.40 \mathrm{E}-01^{b}, 1.54 \mathrm{E}-01^{d}$ & 8.314 & $8.2956^{a}, 8.303^{b}, 8.303^{d}$ & 8.305 & 8.300 \\
\hline 1 & 75 & $5.55 \mathrm{E}-04$ & $4.65 \mathrm{E}-04^{2}$ & $3.00 \mathrm{E}-04^{b}$ & 8.007 & $7.993^{2}, 7.9931^{b}$ & & \\
\hline 1 & 84 & $6.79 \mathrm{E}-05$ & $7.35 \mathrm{E}-05^{2}$ & $6.30 \mathrm{E}-05^{b}$ & 7.937 & $7.927^{2}, 7.9258^{b}$ & & 7.927 \\
\hline 1 & 95 & $5.44 \mathrm{E}-03$ & $0.00 \mathrm{E}+00^{2}$ & $4.60 \mathrm{E}-03^{b}$ & 7.921 & $7.927^{2}, 7.9102^{b}$ & & \\
\hline 1 & 102 & $8.18 \mathrm{E}-02$ & $6.30 \mathrm{E}-02^{1}$ & $6.30 \mathrm{E}-02^{d}$ & 7.480 & $7.480^{d}$ & 7.472 & 7.470 \\
\hline 2 & 7 & $6.21 \mathrm{E}-02$ & $6.43 \mathrm{E}-02^{1}$ & $6.34 \mathrm{E}-02^{b}, 6.43 \mathrm{E}-02^{e}$ & 147.922 & $147.254^{b}, 146.900^{e}$ & 147.240 & 147.300 \\
\hline 2 & 11 & $2.37 \mathrm{E}-02$ & $2.70 \mathrm{E}-02^{1}$ & $2.47 \mathrm{E}-02^{c}, 1.85 \mathrm{E}-02^{f}$ & 11.705 & $11.676^{c}$ & & 11.702 \\
\hline 2 & 17 & 7.47E-01 & $7.50 \mathrm{E}-01^{1}$ & $7.51 \mathrm{E}-01^{c}, 7.7 \mathrm{E}-01^{d}, 6.58 \mathrm{E}-01^{f}$ & 11.320 & $11.299^{c}, 11.298^{d}, 11.285^{g}$ & 11.298 & 11.300 \\
\hline 2 & 54 & $1.38 \mathrm{E}-01$ & $1.40 \mathrm{E}-01^{1}$ & $1.30 \mathrm{E}-01^{a}, 1.30 \mathrm{E}-01^{d}$ & 8.540 & $8.529^{a}, 8.528^{d}$ & 8.529 & 8.530 \\
\hline 3 & 6 & $1.80 \mathrm{E}-02$ & $1.85 \mathrm{E}-02^{1}$ & $1.83 \mathrm{E}-02^{a}$ & 172.469 & $173.318^{a}$ & 173.310 & 173.300 \\
\hline 3 & 11 & $2.43 \mathrm{E}-02$ & $2.70 \mathrm{E}-02^{1}$ & $2.48 \mathrm{E}-02^{c}, 1.89 \mathrm{E}-02^{f}$ & 11.740 & $11.718^{c}, 11.702^{g}$ & 11.748 & 11.740 \\
\hline 3 & 17 & $1.82 \mathrm{E}-01$ & $1.80 \mathrm{E}-01^{1}$ & $1.23 \mathrm{E}-01^{c}, 1.65 \mathrm{E}-01^{f}$ & 11.350 & $11.338^{c}, 11.366^{g}$ & & 11.338 \\
\hline 3 & 23 & $3.14 \mathrm{E}-02$ & $4.90 \mathrm{E}-02^{1}$ & $2.50 \mathrm{E}-01^{c}$ & 11.020 & $11.003^{c}$ & & 11.018 \\
\hline 3 & 54 & 3.37E-02 & $3.50 \mathrm{E}-02^{1}$ & $3.23 \mathrm{E}-02^{a}$ & 8.560 & $8.551^{a}$ & & 8.550 \\
\hline 5 & 23 & $2.48 \mathrm{E}-02$ & $3.00 \mathrm{E}-02^{1}$ & $6.46 \mathrm{E}-06^{c}$ & 11.500 & $11.113^{c}$ & & 11.491 \\
\hline 5 & 27 & $2.59 \mathrm{E}-02$ & $3.30 \mathrm{E}-02^{1}$ & $1.06 \mathrm{E}-03^{c}$ & 11.410 & $11.033^{c}$ & & 11.398 \\
\hline 5 & 48 & $4.17 \mathrm{E}-03$ & $3.70 \mathrm{E}-03^{1}$ & $3.70 \mathrm{E}-03^{d}$ & 8.912 & $8.910^{d}$ & 8.906 & 8.910 \\
\hline 6 & 36 & $1.27 \mathrm{E}+00$ & $1.29 \mathrm{E}+00^{1}$ & $1.27 \mathrm{E}+00^{c}, 1.29 \mathrm{E}+00^{d}$ & 11.508 & $11.486^{c}, 11.489^{d}$ & 11.519 & 11.520 \\
\hline 6 & 69 & $2.34 \mathrm{E}-01$ & $2.30 \mathrm{E}-01^{1}$ & $2.40 \mathrm{E}-01^{a}$ & 8.678 & $8.672^{a}$ & 8.672 & 8.670 \\
\hline 7 & 21 & $2.07 \mathrm{E}-02$ & $1.70 \mathrm{E}-02^{1}$ & & 12.025 & & 12.095 & 12.100 \\
\hline 7 & 36 & $9.15 \mathrm{E}-02$ & $8.90 \mathrm{E}-02^{1}$ & $7.45 \mathrm{E}-04^{c}$ & 11.607 & $11.822^{c}$ & 11.614 & 11.610 \\
\hline 7 & 84 & $5.02 \mathrm{E}-02$ & $4.90 \mathrm{E}-02^{1}$ & & 8.643 & & 8.630 & 8.630 \\
\hline 10 & 25 & $6.44 \mathrm{E}-02$ & $5.50 \mathrm{E}-02^{1}$ & $2.11 \mathrm{E}-03^{c}$ & 12.371 & $12.579^{c}$ & 12.427 & 12.400 \\
\hline 10 & 46 & $1.27 \mathrm{E}+00$ & $1.29 \mathrm{E}+00^{1}$ & $1.28 \mathrm{E}+00^{c}, 1.30 \mathrm{E}+00^{d}$ & 11.898 & $11.873^{c}, 11.840^{d}$ & 11.898 & 11.900 \\
\hline 10 & 75 & $1.53 \mathrm{E}-02$ & $1.77 \mathrm{E}-02^{2}$ & $1.80 \mathrm{E}-02^{d}$ & 9.038 & $9.022^{2}, 9.028^{d}$ & & \\
\hline 10 & 95 & $2.37 \mathrm{E}-01$ & $3.22 \mathrm{E}-02^{2}$ & & 8.929 & $8.935^{2}, 8.920^{h}$ & & \\
\hline
\end{tabular}

${ }^{1,2} f$-values and wavelengths from the NIST [34] and CHIANTI [38] databases, respectively; ${ }^{a}$ Experimental observations (for $\lambda$ ) and benchmark calculations (for $f_{L}$ ) by Del Zanna et al. [26]; ${ }^{b}$ R-matrix calculations by Chidichimo et al. [24,25]; ${ }^{c} \mathrm{MCDF}$ calculations by Jian-Hui et al. [28]; ${ }^{d}$ Compilation of experimental and theoretical data by Fuhr et al. [41]; ${ }^{e}$ MCDF calculations by Cheng et al. [8]; ${ }^{f}$ RQDO calculations by Charro et al. [29]; ${ }^{g}$ Measured wavelengths by Brown et al. [1]; ${ }^{h}$ Measured wavelengths by Chen et al. [2]; $\lambda_{o b s}$ Observed wavelengths from NIST database [34]. Note: For numbers in this paper, $a \mathrm{E} b$ is $a \times 10^{b}$. 
Table 5. Comparison between the present calculations of transition probabilities $\left(A_{L}\right.$ in $\left.s^{-1}\right)$ and other references.

\begin{tabular}{ccccc}
\hline Trans. & Present & Fuhr et al. ${ }^{*}$ & Theoretical & CHIANTI \\
\hline $1-5$ & $1.91 \mathrm{E}+10$ & $1.95 \mathrm{E}+10$ & $1.93 \mathrm{E}+10^{a}$ & $1.94 \mathrm{E}+10$ \\
$1-13$ & $4.99 \mathrm{E}+12$ & $4.90 \mathrm{E}+12$ & $4.65 \mathrm{E}+12^{b}$ & $4.57 \mathrm{E}+12$ \\
$1-15$ & $7.94 \mathrm{E}+12$ & $7.90 \mathrm{E}+12$ & $7.64 \mathrm{E}+12^{b}$ & $7.65 \mathrm{E}+12$ \\
$2-7$ & $6.31 \mathrm{E}+09$ & $6.59 \mathrm{E}+09$ & $6.43 \mathrm{E}+09^{c}$ & $6.49 \mathrm{E}+09$ \\
$2-54$ & $4.19 \mathrm{E}+12$ & $4.30 \mathrm{E}+12$ & $1.23 \mathrm{E}+13^{d}$ & $4.06 \mathrm{E}+12$ \\
$3-6$ & $1.21 \mathrm{E}+10$ & $1.23 \mathrm{E}+10$ & $1.22 \mathrm{E}+10^{c}$ & $1.22 \mathrm{E}+10$ \\
$3-54$ & $2.97 \mathrm{E}+12$ & $3.20 \mathrm{E}+12$ & $2.97 \mathrm{E}+12^{d}$ & $2.95 \mathrm{E}+12$ \\
$3-76$ & $7.03 \mathrm{E}+11$ & $8.30 \mathrm{E}+11$ & $1.86 \mathrm{E}+12^{d}$ & $4.87 \mathrm{E}+11$ \\
$6-69$ & $6.91 \mathrm{E}+12$ & $6.80 \mathrm{E}+12$ & $2.10 \mathrm{E}+13^{d}$ & $7.00 \mathrm{E}+12$ \\
$7-84$ & $4.42 \mathrm{E}+12$ & $4.40 \mathrm{E}+12$ & $4.43 \mathrm{E}+12^{d}$ & $4.41 \mathrm{E}+12$ \\
\hline
\end{tabular}

${ }^{*}$ Compilation of experimental and theoretical data [41]; ${ }^{a}$ Calculated data by Cheng et al. [50]; ${ }^{b}$ Calculated data by Jian-Hui et al. [28]; ${ }^{c}$ Calculated data by Nussbaumer and Storey [42]; ${ }^{d}$ Calculated data by Wyart et al. [51]; CHIANTI: Compilation of experimental and theoretical data by the CHIANTI atomic database [38].

Table 6. Oscillator strengths $f$, wavelengths (in $\AA$ ), transition probabilities $A$ (in $s^{-1}$ ) and line strengths $S$ (in a.u.) for the transitions in Be-like iron. UL. = upper level; LL. $=$ lower level, $V$ and $L$ indicate velocity and length forms.

\begin{tabular}{cccccccc}
\hline LL. & UL. & $\lambda$ & $\boldsymbol{A}_{\boldsymbol{L}}$ & $\boldsymbol{A}_{\boldsymbol{V}}$ & $\boldsymbol{f}_{\boldsymbol{L}}$ & $\boldsymbol{f}_{\boldsymbol{V}}$ & $\boldsymbol{S}_{\boldsymbol{L}}$ \\
\hline 1 & 5 & 132.939 & $1.91 \mathrm{E}+10$ & $1.55 \mathrm{E}+10$ & $1.52 \mathrm{E}-01$ & $1.23 \mathrm{E}-01$ & $6.64 \mathrm{E}-02$ \\
& 13 & 11.036 & $4.99 \mathrm{E}+12$ & $5.30 \mathrm{E}+12$ & $2.73 \mathrm{E}-01$ & $2.90 \mathrm{E}-01$ & $1.05 \mathrm{E}-02$ \\
& 15 & 10.997 & $7.94 \mathrm{E}+12$ & $7.44 \mathrm{E}+12$ & $4.32 \mathrm{E}-01$ & $4.05 \mathrm{E}-01$ & $1.56 \mathrm{E}-02$ \\
& 22 & 10.691 & $1.91 \mathrm{E}+11$ & $2.61 \mathrm{E}+11$ & $9.82 \mathrm{E}-03$ & $1.34 \mathrm{E}-02$ & $3.46 \mathrm{E}-04$ \\
& 36 & 10.369 & $1.24 \mathrm{E}+11$ & $1.20 \mathrm{E}+11$ & $5.97 \mathrm{E}-03$ & $5.80 \mathrm{E}-03$ & $2.04 \mathrm{E}-04$ \\
& 41 & 10.246 & $5.11 \mathrm{E}+09$ & $4.79 \mathrm{E}+09$ & $2.41 \mathrm{E}-04$ & $2.26 \mathrm{E}-04$ & $8.14 \mathrm{E}-06$ \\
& 46 & 10.172 & $4.16 \mathrm{E}+11$ & $4.21 \mathrm{E}+11$ & $1.94 \mathrm{E}-02$ & $1.96 \mathrm{E}-02$ & $6.48 \mathrm{E}-04$ \\
& 49 & 8.326 & $1.20 \mathrm{E}+12$ & $1.11 \mathrm{E}+12$ & $3.73 \mathrm{E}-02$ & $3.47 \mathrm{E}-02$ & $1.02 \mathrm{E}-03$ \\
& 52 & 8.314 & $4.99 \mathrm{E}+12$ & $4.69 \mathrm{E}+12$ & $1.55 \mathrm{E}-01$ & $1.46 \mathrm{E}-01$ & $4.24 \mathrm{E}-03$ \\
& 75 & 8.007 & $1.92 \mathrm{E}+10$ & $1.84 \mathrm{E}+10$ & $5.55 \mathrm{E}-04$ & $5.30 \mathrm{E}-04$ & $1.46 \mathrm{E}-05$ \\
& 84 & 7.937 & $2.40 \mathrm{E}+09$ & $2.33 \mathrm{E}+09$ & $6.79 \mathrm{E}-05$ & $6.60 \mathrm{E}-05$ & $1.77 \mathrm{E}-06$ \\
& 95 & 7.921 & $1.93 \mathrm{E}+11$ & $1.85 \mathrm{E}+11$ & $5.44 \mathrm{E}-03$ & $5.22 \mathrm{E}-03$ & $1.42 \mathrm{E}-04$ \\
& 99 & 7.486 & $5.85 \mathrm{E}+11$ & $4.64 \mathrm{E}+11$ & $1.47 \mathrm{E}-02$ & $1.17 \mathrm{E}-02$ & $3.63 \mathrm{E}-04$ \\
& 102 & 7.480 & $3.25 \mathrm{E}+12$ & $2.53 \mathrm{E}+12$ & $8.18 \mathrm{E}-02$ & $6.38 \mathrm{E}-02$ & $2.02 \mathrm{E}-03$ \\
& 143 & 7.188 & $1.33 \mathrm{E}+09$ & $1.09 \mathrm{E}+09$ & $3.10 \mathrm{E}-05$ & $2.52 \mathrm{E}-05$ & $7.33 \mathrm{E}-07$ \\
& 152 & 7.182 & $9.94 \mathrm{E}+10$ & $8.45 \mathrm{E}+10$ & $2.31 \mathrm{E}-03$ & $1.96 \mathrm{E}-03$ & $5.45 \mathrm{E}-05$ \\
\hline 2 & 7 & 147.922 & $6.31 \mathrm{E}+09$ & $4.59 \mathrm{E}+09$ & $6.21 \mathrm{E}-02$ & $4.52 \mathrm{E}-02$ & $2.20 \mathrm{E}-02$ \\
& 11 & 11.705 & $3.85 \mathrm{E}+11$ & $3.32 \mathrm{E}+11$ & $2.37 \mathrm{E}-02$ & $2.04 \mathrm{E}-02$ & $9.14 \mathrm{E}-04$ \\
\hline
\end{tabular}


Table 6. Cont.

\begin{tabular}{|c|c|c|c|c|c|c|c|}
\hline LL. & UL. & $\lambda$ & $A_{L}$ & $A_{V}$ & $f_{L}$ & $f_{V}$ & $S_{L}$ \\
\hline & 17 & 11.321 & $1.30 \mathrm{E}+13$ & $1.30 \mathrm{E}+13$ & 7.47E-01 & $7.51 \mathrm{E}-01$ & $2.80 \mathrm{E}-02$ \\
\hline & 23 & 10.995 & $8.07 \mathrm{E}+11$ & $1.05 \mathrm{E}+12$ & 4.39E-02 & $5.70 \mathrm{E}-02$ & $2.06 \mathrm{E}-03$ \\
\hline & 27 & 10.913 & $3.10 \mathrm{E}+12$ & $3.23 \mathrm{E}+12$ & $1.66 \mathrm{E}-01$ & $1.73 \mathrm{E}-01$ & $6.21 \mathrm{E}-03$ \\
\hline & 30 & 10.804 & $6.14 \mathrm{E}+11$ & $4.51 \mathrm{E}+11$ & $3.22 \mathrm{E}-02$ & 2.37E-02 & 8.42E-04 \\
\hline & 34 & 10.776 & $1.40 \mathrm{E}+11$ & $7.15 \mathrm{E}+10$ & 7.33E-03 & $3.74 \mathrm{E}-03$ & $1.33 \mathrm{E}-04$ \\
\hline & 47 & 8.627 & $1.89 \mathrm{E}+11$ & $1.21 \mathrm{E}+11$ & $6.34 \mathrm{E}-03$ & $4.05 \mathrm{E}-03$ & $1.15 \mathrm{E}-04$ \\
\hline & 54 & 8.540 & $4.19 \mathrm{E}+12$ & $4.09 \mathrm{E}+12$ & $1.38 \mathrm{E}-01$ & $1.34 \mathrm{E}-01$ & $3.77 \mathrm{E}-03$ \\
\hline & 65 & 8.299 & $8.30 \mathrm{E}+11$ & $8.07 \mathrm{E}+11$ & $2.57 \mathrm{E}-02$ & $2.50 \mathrm{E}-02$ & $6.83 \mathrm{E}-04$ \\
\hline & 76 & 8.280 & $1.94 \mathrm{E}+12$ & $1.85 \mathrm{E}+12$ & $5.99 \mathrm{E}-02$ & $5.69 \mathrm{E}-02$ & $1.55 \mathrm{E}-03$ \\
\hline & 79 & 8.206 & $2.26 \mathrm{E}+10$ & $2.18 \mathrm{E}+10$ & $6.85 \mathrm{E}-04$ & $6.59 \mathrm{E}-04$ & $1.78 \mathrm{E}-05$ \\
\hline & 97 & 8.150 & $5.83 \mathrm{E}+07$ & $2.45 \mathrm{E}+06$ & $1.74 \mathrm{E}-06$ & 7.33E-08 & $1.97 \mathrm{E}-09$ \\
\hline & 118 & 7.463 & $5.50 \mathrm{E}+11$ & $4.57 \mathrm{E}+11$ & $1.38 \mathrm{E}-02$ & $1.14 \mathrm{E}-02$ & $2.81 \mathrm{E}-04$ \\
\hline \multirow[t]{26}{*}{3} & 6 & 172.469 & $1.21 \mathrm{E}+10$ & $7.63 \mathrm{E}+09$ & $1.80 \mathrm{E}-02$ & $1.13 \mathrm{E}-02$ & $3.06 \mathrm{E}-02$ \\
\hline & 8 & 143.720 & $4.05 \mathrm{E}+09$ & $5.31 \mathrm{E}+09$ & $9.89 \mathrm{E}-03$ & $1.30 \mathrm{E}-02$ & $8.21 \mathrm{E}-02$ \\
\hline & 11 & 11.736 & $1.17 \mathrm{E}+12$ & $9.64 \mathrm{E}+11$ & $2.43 \mathrm{E}-02$ & 1.99E-02 & $2.31 \mathrm{E}-03$ \\
\hline & 17 & 11.350 & $9.51 \mathrm{E}+12$ & $9.42 \mathrm{E}+12$ & $1.82 \mathrm{E}-01$ & $1.80 \mathrm{E}-01$ & $2.04 \mathrm{E}-02$ \\
\hline & 18 & 11.348 & $1.71 \mathrm{E}+13$ & $1.70 \mathrm{E}+13$ & $2.06 \mathrm{E}-02$ & $2.05 \mathrm{E}-02$ & $1.64 \mathrm{E}+00$ \\
\hline & 20 & 11.257 & $1.09 \mathrm{E}+11$ & $1.05 \mathrm{E}+11$ & $1.27 \mathrm{E}-04$ & $1.23 \mathrm{E}-04$ & $9.96 \mathrm{E}-03$ \\
\hline & 23 & 11.022 & $2.08 \mathrm{E}+12$ & $1.72 \mathrm{E}+12$ & $3.14 \mathrm{E}-02$ & $2.59 \mathrm{E}-02$ & $3.41 \mathrm{E}-03$ \\
\hline & 26 & 10.946 & $4.15 E+12$ & $3.70 \mathrm{E}+12$ & 4.48E-03 & $4.00 \mathrm{E}-03$ & $3.33 \mathrm{E}-01$ \\
\hline & 27 & 10.940 & $9.30 \mathrm{E}+11$ & $1.02 \mathrm{E}+12$ & $1.84 \mathrm{E}-02$ & $2.01 \mathrm{E}-02$ & $1.99 \mathrm{E}-03$ \\
\hline & 28 & 10.890 & $8.09 \mathrm{E}+12$ & $7.40 \mathrm{E}+12$ & $4.80 \mathrm{E}-02$ & $4.37 \mathrm{E}-02$ & $5.15 \mathrm{E}-03$ \\
\hline & 30 & 10.830 & $2.07 \mathrm{E}+12$ & $2.10 \mathrm{E}+12$ & $3.70 \mathrm{E}-02$ & $3.75 \mathrm{E}-02$ & $3.95 \mathrm{E}-03$ \\
\hline & 37 & 10.727 & $2.43 E+11$ & $2.40 \mathrm{E}+11$ & $2.47 \mathrm{E}-04$ & $2.44 \mathrm{E}-04$ & $2.07 \mathrm{E}-02$ \\
\hline & 44 & 10.626 & $1.20 \mathrm{E}+11$ & $9.83 \mathrm{E}+10$ & $6.77 \mathrm{E}-04$ & $5.53 \mathrm{E}-04$ & $7.08 \mathrm{E}-05$ \\
\hline & 48 & 8.622 & $5.54 \mathrm{E}+10$ & $4.19 \mathrm{E}+10$ & $2.06 \mathrm{E}-04$ & $1.56 \mathrm{E}-04$ & $1.75 \mathrm{E}-05$ \\
\hline & 53 & 8.557 & $5.47 \mathrm{E}+12$ & $5.63 \mathrm{E}+12$ & $2.82 \mathrm{E}-03$ & $2.90 \mathrm{E}-03$ & $3.09 \mathrm{E}-01$ \\
\hline & 54 & 8.556 & $2.97 \mathrm{E}+12$ & $3.06 \mathrm{E}+12$ & $3.37 \mathrm{E}-02$ & $3.47 \mathrm{E}-02$ & $2.84 \mathrm{E}-03$ \\
\hline & 56 & 8.540 & $3.34 \mathrm{E}+10$ & $3.45 \mathrm{E}+10$ & $1.71 \mathrm{E}-05$ & $1.77 \mathrm{E}-05$ & $1.89 \mathrm{E}-03$ \\
\hline & 64 & 8.296 & $2.37 \mathrm{E}+12$ & $2.49 \mathrm{E}+12$ & $1.12 \mathrm{E}-03$ & $1.17 \mathrm{E}-03$ & $1.28 \mathrm{E}-01$ \\
\hline & 65 & 8.295 & $1.32 \mathrm{E}+12$ & $1.46 \mathrm{E}+12$ & $1.51 \mathrm{E}-02$ & $1.67 \mathrm{E}-02$ & $1.24 \mathrm{E}-03$ \\
\hline & 76 & 8.221 & $7.03 \mathrm{E}+11$ & $7.59 \mathrm{E}+11$ & $7.83 \mathrm{E}-03$ & $8.46 \mathrm{E}-03$ & $6.41 \mathrm{E}-04$ \\
\hline & 79 & 8.213 & $5.66 \mathrm{E}+11$ & $5.50 \mathrm{E}+11$ & $5.57 \mathrm{E}-03$ & $5.41 \mathrm{E}-03$ & $4.53 \mathrm{E}-04$ \\
\hline & 80 & 8.200 & $2.80 \mathrm{E}+11$ & $2.82 \mathrm{E}+11$ & $1.27 \mathrm{E}-04$ & $1.28 \mathrm{E}-04$ & $1.42 \mathrm{E}-02$ \\
\hline & 82 & 8.183 & $6.22 \mathrm{E}+11$ & $5.96 \mathrm{E}+11$ & $2.08 \mathrm{E}-03$ & $2.00 \mathrm{E}-03$ & $1.68 \mathrm{E}-04$ \\
\hline & 103 & 7.688 & $2.44 \mathrm{E}+12$ & $2.70 \mathrm{E}+12$ & $9.12 \mathrm{E}-04$ & $1.01 \mathrm{E}-03$ & $1.20 \mathrm{E}-01$ \\
\hline & 105 & 7.681 & $1.70 \mathrm{E}+10$ & $1.87 \mathrm{E}+10$ & $6.34 \mathrm{E}-06$ & $6.99 \mathrm{E}-06$ & $8.29 \mathrm{E}-04$ \\
\hline & 118 & 7.475 & $7.32 \mathrm{E}+11$ & $9.19 \mathrm{E}+11$ & 7.70E-03 & $9.67 \mathrm{E}-03$ & $5.68 \mathrm{E}-04$ \\
\hline
\end{tabular}


Table 6. Cont.

\begin{tabular}{|c|c|c|c|c|c|c|c|}
\hline LL. & UL. & $\lambda$ & $A_{L}$ & $A_{V}$ & $f_{L}$ & $f_{V}$ & $S_{L}$ \\
\hline & 120 & 7.469 & $1.20 \mathrm{E}+12$ & $1.41 \mathrm{E}+12$ & 4.11E-04 & $4.83 \mathrm{E}-04$ & $5.90 \mathrm{E}-02$ \\
\hline & 121 & 7.468 & $4.03 \mathrm{E}+11$ & $5.03 \mathrm{E}+11$ & 4.20E-03 & $5.24 \mathrm{E}-03$ & $3.10 \mathrm{E}-04$ \\
\hline & 137 & 7.402 & $2.56 \mathrm{E}+11$ & $2.53 \mathrm{E}+11$ & $2.08 \mathrm{E}-03$ & $2.06 \mathrm{E}-03$ & $1.52 \mathrm{E}-04$ \\
\hline & 140 & 7.395 & $1.77 \mathrm{E}+11$ & $1.91 \mathrm{E}+11$ & $5.90 \mathrm{E}-05$ & $6.35 \mathrm{E}-05$ & $7.82 \mathrm{E}-03$ \\
\hline \multirow[t]{22}{*}{4} & 11 & 11.867 & $1.62 \mathrm{E}+12$ & $2.01 \mathrm{E}+12$ & $1.03 \mathrm{E}-01$ & $1.27 \mathrm{E}-01$ & 4.01E-03 \\
\hline & 17 & 11.472 & $6.20 \mathrm{E}+11$ & $6.25 \mathrm{E}+11$ & $3.67 \mathrm{E}-02$ & $3.70 \mathrm{E}-02$ & $1.39 \mathrm{E}-03$ \\
\hline & 18 & 11.470 & $5.54 \mathrm{E}+12$ & $5.57 \mathrm{E}+12$ & $5.46 \mathrm{E}-01$ & $5.49 \mathrm{E}-01$ & $2.06 \mathrm{E}-02$ \\
\hline & 20 & 11.377 & $1.90 \mathrm{E}+10$ & $2.02 \mathrm{E}+10$ & $1.85 \mathrm{E}-03$ & $1.96 \mathrm{E}-03$ & $6.91 \mathrm{E}-05$ \\
\hline & 29 & 10.941 & $5.25 \mathrm{E}+11$ & $4.16 \mathrm{E}+11$ & $2.83 \mathrm{E}-02$ & $2.24 \mathrm{E}-02$ & $1.02 \mathrm{E}-03$ \\
\hline & 34 & 10.912 & $5.71 E+12$ & $5.72 \mathrm{E}+12$ & $3.06 \mathrm{E}-01$ & $3.06 \mathrm{E}-01$ & $1.10 \mathrm{E}-02$ \\
\hline & 53 & 8.627 & $1.81 \mathrm{E}+12$ & $1.76 \mathrm{E}+12$ & $1.01 \mathrm{E}-01$ & $9.82 \mathrm{E}-02$ & $2.86 \mathrm{E}-03$ \\
\hline & 54 & 8.626 & $2.01 \mathrm{E}+11$ & $1.98 \mathrm{E}+11$ & $6.73 \mathrm{E}-03$ & $6.62 \mathrm{E}-03$ & $1.91 \mathrm{E}-04$ \\
\hline & 56 & 8.609 & $1.70 \mathrm{E}+10$ & $1.39 \mathrm{E}+10$ & $9.47 \mathrm{E}-04$ & 7.73E-04 & $2.68 \mathrm{E}-05$ \\
\hline & 76 & 8.285 & $6.84 \mathrm{E}+11$ & $6.82 \mathrm{E}+11$ & $2.11 \mathrm{E}-02$ & $2.11 \mathrm{E}-02$ & $5.76 \mathrm{E}-04$ \\
\hline & 77 & 8.282 & $2.29 \mathrm{E}+12$ & $2.25 \mathrm{E}+12$ & $1.18 \mathrm{E}-01$ & $1.16 \mathrm{E}-01$ & $3.21 \mathrm{E}-03$ \\
\hline & 79 & 8.276 & $2.48 \mathrm{E}+12$ & $2.22 \mathrm{E}+12$ & 7.63E-02 & $6.85 \mathrm{E}-02$ & $2.08 \mathrm{E}-03$ \\
\hline & 80 & 8.263 & $7.17 \mathrm{E}+11$ & $7.33 \mathrm{E}+11$ & $3.67 \mathrm{E}-02$ & $3.75 \mathrm{E}-02$ & $9.98 \mathrm{E}-04$ \\
\hline & 93 & 7.744 & $9.80 \mathrm{E}+10$ & $8.90 \mathrm{E}+10$ & $2.64 \mathrm{E}-03$ & $2.40 \mathrm{E}-03$ & $6.73 \mathrm{E}-05$ \\
\hline & 103 & 7.743 & $8.62 \mathrm{E}+11$ & $7.82 \mathrm{E}+11$ & $3.88 \mathrm{E}-02$ & $3.52 \mathrm{E}-02$ & $9.88 \mathrm{E}-04$ \\
\hline & 104 & 7.742 & $9.80 \mathrm{E}+10$ & $8.94 \mathrm{E}+10$ & $2.64 \mathrm{E}-03$ & $2.41 \mathrm{E}-03$ & $6.73 \mathrm{E}-05$ \\
\hline & 105 & 7.737 & $8.83 E+09$ & $7.77 \mathrm{E}+09$ & $3.96 \mathrm{E}-04$ & 3.49E-04 & $1.01 \mathrm{E}-05$ \\
\hline & 121 & 7.520 & $4.71 \mathrm{E}+11$ & $4.21 \mathrm{E}+11$ & $1.18 \mathrm{E}-02$ & $1.05 \mathrm{E}-02$ & $2.89 \mathrm{E}-04$ \\
\hline & 136 & 7.454 & $1.30 \mathrm{E}+12$ & $1.15 \mathrm{E}+12$ & $5.43 \mathrm{E}-02$ & 4.79E-02 & $1.33 \mathrm{E}-03$ \\
\hline & 137 & 7.450 & $1.46 \mathrm{E}+12$ & $1.11 \mathrm{E}+12$ & $3.64 \mathrm{E}-02$ & $2.78 \mathrm{E}-02$ & 8.92E-04 \\
\hline & 140 & 7.447 & $4.36 \mathrm{E}+11$ & $4.02 \mathrm{E}+11$ & $1.81 \mathrm{E}-02$ & $1.67 \mathrm{E}-02$ & 4.44E-04 \\
\hline & 154 & 7.431 & $9.06 \mathrm{E}+09$ & $2.61 \mathrm{E}+09$ & $2.56 \mathrm{E}-05$ & $7.36 \mathrm{E}-06$ & $2.36 \mathrm{E}-06$ \\
\hline \multirow[t]{13}{*}{5} & 8 & 314.333 & $2.76 \mathrm{E}+08$ & $3.49 \mathrm{E}+08$ & $7.05 \mathrm{E}-03$ & 8.91E-03 & $2.58 \mathrm{E}-02$ \\
\hline & 9 & 221.194 & $3.72 \mathrm{E}+09$ & $4.43 \mathrm{E}+09$ & $3.31 \mathrm{E}-02$ & $3.94 \mathrm{E}-02$ & $1.62 \mathrm{E}-01$ \\
\hline & 17 & 11.858 & $1.83 \mathrm{E}+11$ & $1.84 \mathrm{E}+11$ & $3.87 \mathrm{E}-03$ & $3.89 \mathrm{E}-03$ & $4.54 \mathrm{E}-04$ \\
\hline & 18 & 11.856 & $1.08 \mathrm{E}+11$ & $1.08 \mathrm{E}+11$ & $1.48 \mathrm{E}-04$ & $1.48 \mathrm{E}-04$ & $1.14 \mathrm{E}-02$ \\
\hline & 20 & 11.757 & $1.64 \mathrm{E}+13$ & $1.61 \mathrm{E}+13$ & $2.19 \mathrm{E}-02$ & $2.15 \mathrm{E}-02$ & $1.66 \mathrm{E}+00$ \\
\hline & 23 & 11.501 & $1.36 \mathrm{E}+12$ & $1.25 \mathrm{E}+12$ & $2.48 \mathrm{E}-02$ & $2.28 \mathrm{E}-02$ & $2.82 \mathrm{E}-03$ \\
\hline & 26 & 11.418 & $3.53 \mathrm{E}+11$ & $4.32 \mathrm{E}+11$ & 4.32E-04 & $5.28 \mathrm{E}-04$ & $4.22 \mathrm{E}-02$ \\
\hline & 27 & 11.411 & $1.49 \mathrm{E}+12$ & $1.33 \mathrm{E}+12$ & $2.59 \mathrm{E}-02$ & $2.31 \mathrm{E}-02$ & $2.92 \mathrm{E}-03$ \\
\hline & 28 & 11.357 & $2.43 \mathrm{E}+11$ & $2.48 \mathrm{E}+11$ & $1.57 \mathrm{E}-03$ & $1.60 \mathrm{E}-03$ & $1.76 \mathrm{E}-04$ \\
\hline & 30 & 11.292 & $3.38 \mathrm{E}+12$ & $3.13 \mathrm{E}+12$ & $5.97 \mathrm{E}-02$ & $5.53 \mathrm{E}-02$ & $6.66 \mathrm{E}-03$ \\
\hline & 34 & 11.261 & $3.36 \mathrm{E}+11$ & $3.02 \mathrm{E}+11$ & $5.73 \mathrm{E}-03$ & $5.15 \mathrm{E}-03$ & $6.38 \mathrm{E}-04$ \\
\hline & 35 & 11.260 & $2.04 \mathrm{E}+12$ & $2.23 E+12$ & 2.39E-03 & $2.61 \mathrm{E}-03$ & $2.12 \mathrm{E}-01$ \\
\hline & 37 & 11.180 & $6.84 \mathrm{E}+12$ & $7.63 \mathrm{E}+12$ & 7.86E-03 & 8.77E-03 & 7.15E-01 \\
\hline
\end{tabular}


Table 6. Cont.

\begin{tabular}{|c|c|c|c|c|c|c|c|}
\hline LL. & UL. & $\lambda$ & $A_{L}$ & $A_{V}$ & $f_{L}$ & $f_{V}$ & $S_{L}$ \\
\hline & 44 & 11.070 & $7.53 \mathrm{E}+12$ & $7.26 \mathrm{E}+12$ & 4.60E-02 & $4.43 \mathrm{E}-02$ & $5.04 \mathrm{E}-03$ \\
\hline & 47 & 8.935 & $9.40 \mathrm{E}+09$ & $1.23 \mathrm{E}+10$ & $1.47 \mathrm{E}-04$ & $1.93 \mathrm{E}-04$ & $1.30 \mathrm{E}-05$ \\
\hline & 48 & 8.912 & $1.05 \mathrm{E}+12$ & $8.33 \mathrm{E}+11$ & 4.17E-03 & $3.31 \mathrm{E}-03$ & $3.66 \mathrm{E}-04$ \\
\hline & 53 & 8.843 & $1.44 \mathrm{E}+10$ & $1.43 \mathrm{E}+10$ & $8.18 \mathrm{E}-06$ & $8.15 \mathrm{E}-06$ & $8.40 \mathrm{E}-04$ \\
\hline & 54 & 8.842 & $6.54 \mathrm{E}+10$ & $6.63 \mathrm{E}+10$ & 7.77E-04 & $7.87 \mathrm{E}-04$ & $6.79 \mathrm{E}-05$ \\
\hline & 56 & 8.825 & $6.25 \mathrm{E}+12$ & $6.36 \mathrm{E}+12$ & $3.53 \mathrm{E}-03$ & $3.59 \mathrm{E}-03$ & $3.71 \mathrm{E}-01$ \\
\hline & 64 & 8.564 & $4.47 \mathrm{E}+11$ & $4.62 \mathrm{E}+11$ & $2.31 \mathrm{E}-04$ & 2.39E-04 & $2.54 \mathrm{E}-02$ \\
\hline & 65 & 8.563 & $2.83 \mathrm{E}+11$ & $3.53 \mathrm{E}+11$ & $3.90 \mathrm{E}-03$ & $4.86 \mathrm{E}-03$ & $3.29 \mathrm{E}-04$ \\
\hline & 76 & 8.484 & $1.63 \mathrm{E}+12$ & $1.95 \mathrm{E}+12$ & $2.10 \mathrm{E}-02$ & $2.51 \mathrm{E}-02$ & $1.76 \mathrm{E}-03$ \\
\hline & 77 & 8.481 & $8.04 \mathrm{E}+11$ & $8.28 \mathrm{E}+11$ & 4.04E-04 & $4.15 \mathrm{E}-04$ & 4.46E-02 \\
\hline & 79 & 8.476 & $4.64 \mathrm{E}+11$ & $5.68 \mathrm{E}+11$ & $6.10 \mathrm{E}-03$ & 7.47E-03 & $5.12 \mathrm{E}-04$ \\
\hline & 80 & 8.462 & $2.28 \mathrm{E}+12$ & $2.35 \mathrm{E}+12$ & $1.14 \mathrm{E}-03$ & $1.17 \mathrm{E}-03$ & $1.26 \mathrm{E}-01$ \\
\hline & 82 & 8.444 & $2.28 \mathrm{E}+12$ & $2.74 \mathrm{E}+12$ & $8.13 \mathrm{E}-03$ & $9.77 \mathrm{E}-03$ & $6.77 \mathrm{E}-04$ \\
\hline & 89 & 8.432 & $1.07 \mathrm{E}+10$ & $1.23 \mathrm{E}+10$ & $5.28 \mathrm{E}-06$ & $6.09 \mathrm{E}-06$ & $6.58 \mathrm{E}-04$ \\
\hline & 93 & 8.425 & $5.95 \mathrm{E}+07$ & $5.63 \mathrm{E}+07$ & $6.00 \mathrm{E}-07$ & $5.68 \mathrm{E}-07$ & $4.98 \mathrm{E}-08$ \\
\hline & 94 & 8.425 & $1.73 \mathrm{E}+10$ & $2.16 \mathrm{E}+10$ & 8.52E-06 & $1.06 \mathrm{E}-05$ & $1.15 \mathrm{E}-03$ \\
\hline & 103 & 7.918 & $3.17 \mathrm{E}+09$ & $3.51 \mathrm{E}+09$ & $1.29 \mathrm{E}-06$ & $1.43 \mathrm{E}-06$ & $1.65 \mathrm{E}-04$ \\
\hline & 105 & 7.911 & $3.11 \mathrm{E}+12$ & $3.31 \mathrm{E}+12$ & $1.27 \mathrm{E}-03$ & $1.35 \mathrm{E}-03$ & $1.55 \mathrm{E}-01$ \\
\hline & 120 & 7.686 & $3.01 \mathrm{E}+11$ & $3.50 \mathrm{E}+11$ & $1.12 \mathrm{E}-04$ & $1.31 \mathrm{E}-04$ & $1.55 \mathrm{E}-02$ \\
\hline & 121 & 7.684 & $1.09 \mathrm{E}+11$ & $1.89 \mathrm{E}+11$ & $1.67 \mathrm{E}-03$ & $2.90 \mathrm{E}-03$ & $1.27 \mathrm{E}-04$ \\
\hline & 136 & 7.615 & $3.96 \mathrm{E}+11$ & $4.61 \mathrm{E}+11$ & $1.44 \mathrm{E}-04$ & $1.68 \mathrm{E}-04$ & $2.01 \mathrm{E}-02$ \\
\hline & 140 & 7.608 & $1.09 \mathrm{E}+12$ & $1.27 \mathrm{E}+12$ & $3.96 \mathrm{E}-04$ & 4.60E-04 & $5.51 \mathrm{E}-02$ \\
\hline \multirow[t]{10}{*}{6} & 22 & 11.906 & $1.09 \mathrm{E}+12$ & $9.02 \mathrm{E}+11$ & $6.93 \mathrm{E}-02$ & $5.75 \mathrm{E}-02$ & $2.72 \mathrm{E}-03$ \\
\hline & 25 & 11.690 & $4.03 E+10$ & $3.58 \mathrm{E}+10$ & $2.48 \mathrm{E}-03$ & $2.20 \mathrm{E}-03$ & $9.53 \mathrm{E}-05$ \\
\hline & 36 & 11.508 & $2.14 \mathrm{E}+13$ & $2.12 \mathrm{E}+13$ & $1.27 \mathrm{E}+00$ & $1.26 \mathrm{E}+00$ & 4.83E-02 \\
\hline & 41 & 11.357 & $3.10 \mathrm{E}+10$ & $3.25 \mathrm{E}+10$ & $1.80 \mathrm{E}-03$ & $1.89 \mathrm{E}-03$ & $6.73 \mathrm{E}-05$ \\
\hline & 46 & 11.266 & $4.43 \mathrm{E}+11$ & $4.54 \mathrm{E}+11$ & $2.53 \mathrm{E}-02$ & $2.59 \mathrm{E}-02$ & $9.37 \mathrm{E}-04$ \\
\hline & 69 & 8.678 & $6.91 \mathrm{E}+12$ & $5.51 \mathrm{E}+12$ & 2.34E-01 & $1.87 \mathrm{E}-01$ & $5.33 \mathrm{E}-03$ \\
\hline & 75 & 8.669 & $1.23 \mathrm{E}+11$ & $1.11 \mathrm{E}+11$ & 4.16E-03 & $3.76 \mathrm{E}-03$ & 1.19E-04 \\
\hline & 84 & 8.588 & $2.26 \mathrm{E}+09$ & $1.95 \mathrm{E}+09$ & 7.49E-05 & $6.45 \mathrm{E}-05$ & $2.12 \mathrm{E}-06$ \\
\hline & 95 & 8.569 & $5.62 \mathrm{E}+11$ & $5.11 \mathrm{E}+11$ & $1.86 \mathrm{E}-02$ & $1.69 \mathrm{E}-02$ & $5.23 \mathrm{E}-04$ \\
\hline & 152 & 7.710 & $3.35 \mathrm{E}+11$ & $3.09 \mathrm{E}+11$ & 8.95E-03 & 8.27E-03 & $2.27 \mathrm{E}-04$ \\
\hline \multirow[t]{7}{*}{7} & 21 & 12.025 & $2.87 \mathrm{E}+12$ & $2.54 \mathrm{E}+12$ & $2.07 \mathrm{E}-02$ & $1.83 \mathrm{E}-02$ & $2.18 \mathrm{E}-03$ \\
\hline & 22 & 12.012 & $5.70 \mathrm{E}+11$ & $4.96 \mathrm{E}+11$ & $1.42 \mathrm{E}-02$ & $1.23 \mathrm{E}-02$ & $1.46 \mathrm{E}-03$ \\
\hline & 24 & 11.857 & $1.01 \mathrm{E}+12$ & $1.13 \mathrm{E}+12$ & $3.56 \mathrm{E}-02$ & $3.98 \mathrm{E}-02$ & $4.66 \mathrm{E}-03$ \\
\hline & 25 & 11.792 & $1.20 \mathrm{E}+11$ & $1.04 \mathrm{E}+11$ & $2.89 \mathrm{E}-03$ & $2.51 \mathrm{E}-03$ & $2.92 \mathrm{E}-04$ \\
\hline & 29 & 11.707 & $2.44 \mathrm{E}+11$ & $2.45 \mathrm{E}+11$ & 8.34E-03 & 8.39E-03 & $9.70 \mathrm{E}-04$ \\
\hline & 33 & 11.631 & $1.27 \mathrm{E}+13$ & $1.27 \mathrm{E}+13$ & 4.29E-01 & $4.29 \mathrm{E}-01$ & 4.93E-02 \\
\hline & 36 & 11.607 & $4.53 \mathrm{E}+12$ & $4.52 \mathrm{E}+12$ & $9.15 \mathrm{E}-02$ & $9.13 \mathrm{E}-02$ & $1.05 \mathrm{E}-02$ \\
\hline
\end{tabular}


Table 6. Cont.

\begin{tabular}{|c|c|c|c|c|c|c|c|}
\hline LL. & UL. & $\lambda$ & $A_{L}$ & $A_{V}$ & $f_{L}$ & $f_{V}$ & $S_{L}$ \\
\hline & 38 & 11.511 & $1.08 \mathrm{E}+13$ & $1.08 \mathrm{E}+13$ & $3.57 \mathrm{E}-01$ & $3.57 \mathrm{E}-01$ & $4.06 \mathrm{E}-02$ \\
\hline & 41 & 11.453 & $1.17 \mathrm{E}+13$ & $1.17 \mathrm{E}+13$ & $2.30 \mathrm{E}-01$ & $2.30 \mathrm{E}-01$ & $2.60 \mathrm{E}-02$ \\
\hline & 42 & 11.452 & $2.07 \mathrm{E}+12$ & $2.07 \mathrm{E}+12$ & $6.79 \mathrm{E}-02$ & $6.77 \mathrm{E}-02$ & $7.66 \mathrm{E}-03$ \\
\hline & 43 & 11.438 & $1.60 \mathrm{E}+13$ & $1.62 \mathrm{E}+13$ & $1.04 \mathrm{E}-01$ & $1.06 \mathrm{E}-01$ & $1.19 \mathrm{E}-02$ \\
\hline & 46 & 11.361 & $2.51 \mathrm{E}+11$ & $2.51 \mathrm{E}+11$ & $4.87 \mathrm{E}-03$ & $4.87 \mathrm{E}-03$ & $5.46 \mathrm{E}-04$ \\
\hline & 52 & 9.091 & $1.59 \mathrm{E}+07$ & $1.60 \mathrm{E}+07$ & $1.95 \mathrm{E}-07$ & $1.96 \mathrm{E}-07$ & $1.76 \mathrm{E}-08$ \\
\hline & 57 & 9.041 & $9.09 \mathrm{E}+06$ & $8.81 \mathrm{E}+06$ & $1.86 \mathrm{E}-07$ & $1.80 \mathrm{E}-07$ & $1.61 \mathrm{E}-08$ \\
\hline & 66 & 8.754 & $6.74 \mathrm{E}+10$ & $6.59 \mathrm{E}+10$ & $1.29 \mathrm{E}-03$ & $1.26 \mathrm{E}-03$ & $1.09 \mathrm{E}-04$ \\
\hline & 67 & 8.741 & $5.40 \mathrm{E}+12$ & $5.61 \mathrm{E}+12$ & $1.03 \mathrm{E}-01$ & $1.07 \mathrm{E}-01$ & $9.24 \mathrm{E}-03$ \\
\hline & 69 & 8.734 & $1.24 \mathrm{E}+12$ & $1.22 \mathrm{E}+12$ & $1.45 \mathrm{E}-02$ & $1.42 \mathrm{E}-02$ & $1.23 \mathrm{E}-03$ \\
\hline & 71 & 8.729 & $6.18 \mathrm{E}+11$ & $4.94 \mathrm{E}+11$ & $1.18 \mathrm{E}-02$ & $9.40 \mathrm{E}-03$ & $8.10 \mathrm{E}-04$ \\
\hline & 81 & 8.654 & $2.27 \mathrm{E}+12$ & $2.30 \mathrm{E}+12$ & $4.25 \mathrm{E}-02$ & $4.30 \mathrm{E}-02$ & $3.67 \mathrm{E}-03$ \\
\hline & 84 & 8.643 & $4.42 \mathrm{E}+12$ & $4.37 \mathrm{E}+12$ & $5.02 \mathrm{E}-02$ & 4.97E-02 & $4.23 \mathrm{E}-03$ \\
\hline & 85 & 8.642 & $9.54 \mathrm{E}+11$ & $9.80 \mathrm{E}+11$ & $1.78 \mathrm{E}-02$ & $1.83 \mathrm{E}-02$ & $1.56 \mathrm{E}-03$ \\
\hline & 88 & 8.637 & $5.66 \mathrm{E}+12$ & $5.52 \mathrm{E}+12$ & $2.11 \mathrm{E}-02$ & $2.06 \mathrm{E}-02$ & $1.75 \mathrm{E}-03$ \\
\hline & 95 & 8.623 & $1.44 \mathrm{E}+11$ & $1.41 \mathrm{E}+11$ & $1.64 \mathrm{E}-03$ & $1.60 \mathrm{E}-03$ & $1.36 \mathrm{E}-04$ \\
\hline & 122 & 7.844 & $4.79 \mathrm{E}+10$ & $5.17 \mathrm{E}+10$ & 7.36E-04 & 7.94E-04 & $6.15 \mathrm{E}-05$ \\
\hline & 124 & 7.838 & $2.83 \mathrm{E}+12$ & $3.14 \mathrm{E}+12$ & $4.34 \mathrm{E}-02$ & $4.82 \mathrm{E}-02$ & $3.73 \mathrm{E}-03$ \\
\hline & 125 & 7.837 & $5.53 \mathrm{E}+11$ & $4.95 \mathrm{E}+11$ & $5.70 \mathrm{E}-03$ & $5.10 \mathrm{E}-03$ & $3.94 \mathrm{E}-04$ \\
\hline & 141 & 7.765 & $9.50 \mathrm{E}+11$ & $1.05 \mathrm{E}+12$ & $1.43 \mathrm{E}-02$ & $1.58 \mathrm{E}-02$ & $1.21 \mathrm{E}-03$ \\
\hline & 143 & 7.761 & $2.29 \mathrm{E}+12$ & $2.05 \mathrm{E}+12$ & $2.30 \mathrm{E}-02$ & $2.06 \mathrm{E}-02$ & $1.58 \mathrm{E}-03$ \\
\hline & 144 & 7.761 & $4.79 \mathrm{E}+11$ & $5.35 \mathrm{E}+11$ & $7.21 \mathrm{E}-03$ & $8.05 \mathrm{E}-03$ & $6.17 \mathrm{E}-04$ \\
\hline & 149 & 7.756 & $3.10 \mathrm{E}+12$ & $2.84 \mathrm{E}+12$ & $9.33 \mathrm{E}-03$ & $8.53 \mathrm{E}-03$ & $6.54 \mathrm{E}-04$ \\
\hline & 152 & 7.754 & $9.19 \mathrm{E}+10$ & $8.10 \mathrm{E}+10$ & $9.42 \mathrm{E}-04$ & 8.30E-04 & $6.35 \mathrm{E}-05$ \\
\hline & 153 & 7.754 & $3.56 \mathrm{E}+08$ & $3.92 \mathrm{E}+08$ & $5.34 \mathrm{E}-06$ & $5.89 \mathrm{E}-06$ & $4.51 \mathrm{E}-07$ \\
\hline \multirow[t]{14}{*}{8} & 22 & 12.073 & $1.29 \mathrm{E}+12$ & $1.36 \mathrm{E}+12$ & $3.35 \mathrm{E}-03$ & $3.55 \mathrm{E}-03$ & 8.94E-02 \\
\hline & 24 & 11.917 & $1.97 \mathrm{E}+12$ & $1.77 \mathrm{E}+12$ & $2.10 \mathrm{E}-01$ & $1.88 \mathrm{E}-01$ & $8.22 \mathrm{E}-03$ \\
\hline & 25 & 11.851 & $1.11 \mathrm{E}+11$ & $1.02 \mathrm{E}+11$ & $2.73 \mathrm{E}-04$ & $2.51 \mathrm{E}-04$ & $6.42 \mathrm{E}-03$ \\
\hline & 29 & 11.765 & $1.60 \mathrm{E}+12$ & $1.60 \mathrm{E}+12$ & $1.66 \mathrm{E}-01$ & $1.66 \mathrm{E}-01$ & $6.41 \mathrm{E}-03$ \\
\hline & 31 & 11.699 & $6.84 \mathrm{E}+12$ & $6.88 \mathrm{E}+12$ & $9.82 \mathrm{E}-01$ & $9.88 \mathrm{E}-01$ & $3.78 \mathrm{E}-02$ \\
\hline & 33 & 11.689 & $1.55 \mathrm{E}+12$ & $1.56 \mathrm{E}+12$ & $1.59 \mathrm{E}-01$ & $1.60 \mathrm{E}-01$ & $6.12 \mathrm{E}-03$ \\
\hline & 36 & 11.664 & $1.84 \mathrm{E}+11$ & $1.82 \mathrm{E}+11$ & $4.31 \mathrm{E}-04$ & $4.29 \mathrm{E}-04$ & $1.12 \mathrm{E}-02$ \\
\hline & 38 & 11.567 & $2.15 \mathrm{E}+12$ & $2.16 \mathrm{E}+12$ & $2.16 \mathrm{E}-01$ & $2.17 \mathrm{E}-01$ & $8.21 \mathrm{E}-03$ \\
\hline & 40 & 11.526 & $2.30 \mathrm{E}+13$ & $2.31 \mathrm{E}+13$ & $3.21 \mathrm{E}+00$ & $3.23 \mathrm{E}+00$ & $1.22 \mathrm{E}-01$ \\
\hline & 41 & 11.508 & $5.61 E+12$ & $5.61 \mathrm{E}+12$ & $1.27 \mathrm{E}-02$ & $1.27 \mathrm{E}-02$ & 3.34E-01 \\
\hline & 42 & 11.508 & $1.13 \mathrm{E}+13$ & $1.13 \mathrm{E}+13$ & $1.12 \mathrm{E}+00$ & $1.12 \mathrm{E}+00$ & $4.25 \mathrm{E}-02$ \\
\hline & 45 & 11.419 & $2.19 \mathrm{E}+12$ & $2.24 \mathrm{E}+12$ & $3.00 \mathrm{E}-01$ & $3.06 \mathrm{E}-01$ & $1.13 \mathrm{E}-02$ \\
\hline & 46 & 11.415 & $1.40 \mathrm{E}+10$ & $1.60 \mathrm{E}+10$ & $3.07 \mathrm{E}-05$ & $3.51 \mathrm{E}-05$ & $9.35 \mathrm{E}-04$ \\
\hline & 57 & 9.075 & $7.03 \mathrm{E}+06$ & $7.10 \mathrm{E}+06$ & 4.34E-07 & $4.38 \mathrm{E}-07$ & $1.30 \mathrm{E}-08$ \\
\hline
\end{tabular}


Table 6. Cont.

\begin{tabular}{|c|c|c|c|c|c|c|c|}
\hline LL. & UL. & $\lambda$ & $A_{L}$ & $A_{V}$ & $f_{L}$ & $f_{V}$ & $S_{L}$ \\
\hline & 66 & 8.786 & $8.08 \mathrm{E}+11$ & $7.85 \mathrm{E}+11$ & 4.68E-02 & 4.54E-02 & $1.35 \mathrm{E}-03$ \\
\hline & 67 & 8.773 & $1.02 \mathrm{E}+12$ & $8.87 \mathrm{E}+11$ & 5.88E-02 & $5.12 \mathrm{E}-02$ & $1.70 \mathrm{E}-03$ \\
\hline & 68 & 8.766 & $4.57 \mathrm{E}+12$ & $4.52 \mathrm{E}+12$ & $3.68 \mathrm{E}-01$ & 3.64E-01 & $1.06 \mathrm{E}-02$ \\
\hline & 69 & 8.766 & $1.11 \mathrm{E}+11$ & $1.18 \mathrm{E}+11$ & $1.11 \mathrm{E}-04$ & $1.18 \mathrm{E}-04$ & $4.09 \mathrm{E}-03$ \\
\hline & 81 & 8.685 & $9.50 \mathrm{E}+11$ & $9.02 \mathrm{E}+11$ & 5.37E-02 & $5.10 \mathrm{E}-02$ & $1.54 \mathrm{E}-03$ \\
\hline & 83 & 8.675 & $6.62 \mathrm{E}+12$ & $6.53 \mathrm{E}+12$ & $5.23 \mathrm{E}-01$ & $5.16 \mathrm{E}-01$ & $1.49 \mathrm{E}-02$ \\
\hline & 84 & 8.674 & $1.92 \mathrm{E}+12$ & $1.96 \mathrm{E}+12$ & $1.86 \mathrm{E}-03$ & 1.89E-03 & $6.62 \mathrm{E}-02$ \\
\hline & 85 & 8.673 & $3.65 \mathrm{E}+12$ & $3.67 \mathrm{E}+12$ & $2.06 \mathrm{E}-01$ & $2.07 \mathrm{E}-01$ & $5.88 \mathrm{E}-03$ \\
\hline & 96 & 8.654 & $2.06 \mathrm{E}+11$ & $2.11 \mathrm{E}+11$ & $1.62 \mathrm{E}-02$ & $1.66 \mathrm{E}-02$ & $4.62 \mathrm{E}-04$ \\
\hline & 122 & 7.870 & $3.92 \mathrm{E}+11$ & $3.63 \mathrm{E}+11$ & $1.82 \mathrm{E}-02$ & $1.68 \mathrm{E}-02$ & $4.72 \mathrm{E}-04$ \\
\hline & 124 & 7.864 & $3.04 \mathrm{E}+11$ & $2.68 \mathrm{E}+11$ & $1.41 \mathrm{E}-02$ & $1.24 \mathrm{E}-02$ & $3.65 \mathrm{E}-04$ \\
\hline & 125 & 7.863 & $5.53 \mathrm{E}+10$ & $7.19 \mathrm{E}+10$ & $3.98 \mathrm{E}-05$ & $5.18 \mathrm{E}-05$ & $2.00 \mathrm{E}-03$ \\
\hline & 129 & 7.860 & $2.74 \mathrm{E}+12$ & $2.54 \mathrm{E}+12$ & $1.78 \mathrm{E}-01$ & $1.65 \mathrm{E}-01$ & $4.60 \mathrm{E}-03$ \\
\hline & 131 & 7.856 & $5.67 \mathrm{E}+09$ & $5.19 \mathrm{E}+09$ & 3.67E-04 & $3.36 \mathrm{E}-04$ & $9.49 \mathrm{E}-06$ \\
\hline & 141 & 7.791 & $4.88 \mathrm{E}+11$ & $4.47 \mathrm{E}+11$ & 2.22E-02 & $2.03 \mathrm{E}-02$ & $5.69 \mathrm{E}-04$ \\
\hline & 143 & 7.787 & $8.81 E+11$ & $9.93 E+11$ & $6.16 \mathrm{E}-04$ & $6.94 \mathrm{E}-04$ & $2.71 \mathrm{E}-02$ \\
\hline & 146 & 7.785 & $3.19 \mathrm{E}+12$ & $2.93 \mathrm{E}+12$ & 2.03E-01 & $1.86 \mathrm{E}-01$ & $5.20 \mathrm{E}-03$ \\
\hline & 157 & 7.778 & $4.78 \mathrm{E}+08$ & $4.48 \mathrm{E}+08$ & 3.03E-05 & $2.85 \mathrm{E}-05$ & 7.77E-07 \\
\hline & 159 & 7.776 & $4.74 \mathrm{E}+10$ & $4.29 \mathrm{E}+10$ & $3.01 \mathrm{E}-03$ & $2.73 \mathrm{E}-03$ & 7.70E-05 \\
\hline & 161 & 7.776 & $1.02 \mathrm{E}+10$ & $9.31 \mathrm{E}+09$ & $6.50 \mathrm{E}-04$ & $5.91 \mathrm{E}-04$ & $1.66 \mathrm{E}-05$ \\
\hline \multirow[t]{19}{*}{9} & 24 & 12.110 & $5.12 \mathrm{E}+11$ & $4.62 \mathrm{E}+11$ & $5.63 \mathrm{E}-02$ & $5.08 \mathrm{E}-02$ & $2.24 \mathrm{E}-03$ \\
\hline & 25 & 12.042 & $2.26 \mathrm{E}+12$ & $2.29 \mathrm{E}+12$ & $5.83 \mathrm{E}-03$ & $5.93 \mathrm{E}-03$ & $1.49 \mathrm{E}-01$ \\
\hline & 29 & 11.953 & $8.02 \mathrm{E}+11$ & $7.92 \mathrm{E}+11$ & 8.59E-02 & $8.48 \mathrm{E}-02$ & $3.38 \mathrm{E}-03$ \\
\hline & 31 & 11.885 & $2.16 \mathrm{E}+11$ & $2.23 E+11$ & $3.21 \mathrm{E}-02$ & $3.30 \mathrm{E}-02$ & $1.25 \mathrm{E}-03$ \\
\hline & 33 & 11.875 & $1.83 \mathrm{E}+12$ & $1.82 \mathrm{E}+12$ & $1.93 \mathrm{E}-01$ & $1.92 \mathrm{E}-01$ & $7.56 \mathrm{E}-03$ \\
\hline & 36 & 11.849 & $3.47 \mathrm{E}+10$ & $3.63 \mathrm{E}+10$ & $8.55 \mathrm{E}-05$ & $8.95 \mathrm{E}-05$ & $2.29 \mathrm{E}-03$ \\
\hline & 38 & 11.750 & $2.42 \mathrm{E}+12$ & $2.39 \mathrm{E}+12$ & $2.51 \mathrm{E}-01$ & $2.48 \mathrm{E}-01$ & $9.70 \mathrm{E}-03$ \\
\hline & 40 & 11.707 & $1.60 \mathrm{E}+12$ & $1.59 \mathrm{E}+12$ & $2.30 \mathrm{E}-01$ & $2.28 \mathrm{E}-01$ & $8.85 \mathrm{E}-03$ \\
\hline & 41 & 11.688 & $1.51 \mathrm{E}+12$ & $1.51 \mathrm{E}+12$ & $3.58 \mathrm{E}-03$ & $3.56 \mathrm{E}-03$ & $9.25 \mathrm{E}-02$ \\
\hline & 42 & 11.688 & $7.20 \mathrm{E}+12$ & $7.20 \mathrm{E}+12$ & 7.37E-01 & 7.37E-01 & $2.84 \mathrm{E}-02$ \\
\hline & 45 & 11.597 & $3.44 \mathrm{E}+13$ & $3.48 \mathrm{E}+13$ & $4.85 \mathrm{E}+00$ & $4.91 \mathrm{E}+00$ & $1.85 \mathrm{E}-01$ \\
\hline & 46 & 11.593 & $9.81 \mathrm{E}+11$ & $1.01 \mathrm{E}+12$ & $2.26 \mathrm{E}-03$ & $2.32 \mathrm{E}-03$ & $6.08 \mathrm{E}-02$ \\
\hline & 52 & 9.239 & $4.45 \mathrm{E}+09$ & $3.46 \mathrm{E}+10$ & 5.19E-06 & 4.04E-05 & $1.33 \mathrm{E}-03$ \\
\hline & 57 & 9.187 & $1.71 \mathrm{E}+06$ & $1.78 \mathrm{E}+06$ & $1.08 \mathrm{E}-07$ & $1.13 \mathrm{E}-07$ & $3.27 \mathrm{E}-09$ \\
\hline & 58 & 9.182 & $2.16 \mathrm{E}+08$ & $1.65 \mathrm{E}+08$ & $1.91 \mathrm{E}-05$ & $1.46 \mathrm{E}-05$ & $5.78 \mathrm{E}-07$ \\
\hline & 59 & 9.177 & $8.24 \mathrm{E}+09$ & $6.44 \mathrm{E}+09$ & 7.28E-04 & $5.70 \mathrm{E}-04$ & $2.20 \mathrm{E}-05$ \\
\hline & 66 & 8.891 & $1.87 \mathrm{E}+11$ & $1.90 \mathrm{E}+11$ & $1.11 \mathrm{E}-02$ & $1.13 \mathrm{E}-02$ & $3.24 \mathrm{E}-04$ \\
\hline & 67 & 8.878 & $2.61 \mathrm{E}+10$ & $3.33 \mathrm{E}+10$ & $1.54 \mathrm{E}-03$ & 1.97E-03 & $4.50 \mathrm{E}-05$ \\
\hline & 68 & 8.870 & $5.02 \mathrm{E}+11$ & $5.06 \mathrm{E}+11$ & $4.15 \mathrm{E}-02$ & 4.18E-02 & $1.21 \mathrm{E}-03$ \\
\hline
\end{tabular}


Table 6. Cont.

\begin{tabular}{|c|c|c|c|c|c|c|c|}
\hline LL. & UL. & $\lambda$ & $A_{L}$ & $A_{V}$ & $f_{L}$ & $f_{V}$ & $S_{L}$ \\
\hline & 69 & 8.870 & $3.48 \mathrm{E}+10$ & $4.49 \mathrm{E}+10$ & $3.59 \mathrm{E}-05$ & $4.64 \mathrm{E}-05$ & $1.59 \mathrm{E}-03$ \\
\hline & 71 & 8.865 & $4.88 \mathrm{E}+11$ & $3.99 \mathrm{E}+11$ & $2.87 \mathrm{E}-02$ & $2.35 \mathrm{E}-02$ & 8.38E-04 \\
\hline & 81 & 8.788 & $1.06 \mathrm{E}+12$ & $1.05 \mathrm{E}+12$ & $6.11 \mathrm{E}-02$ & $6.06 \mathrm{E}-02$ & $1.77 \mathrm{E}-03$ \\
\hline & 83 & 8.777 & $3.09 \mathrm{E}+11$ & $3.00 \mathrm{E}+11$ & $2.50 \mathrm{E}-02$ & $2.42 \mathrm{E}-02$ & 7.22E-04 \\
\hline & 84 & 8.776 & $5.02 \mathrm{E}+11$ & $4.98 \mathrm{E}+11$ & $5.02 \mathrm{E}-04$ & $4.99 \mathrm{E}-04$ & $1.73 \mathrm{E}-02$ \\
\hline & 85 & 8.775 & $2.72 \mathrm{E}+12$ & $2.66 \mathrm{E}+12$ & $1.57 \mathrm{E}-01$ & $1.54 \mathrm{E}-01$ & 4.53E-03 \\
\hline & 95 & 8.756 & $3.32 \mathrm{E}+11$ & $3.65 E+11$ & $3.30 \mathrm{E}-04$ & $3.63 \mathrm{E}-04$ & $1.26 \mathrm{E}-02$ \\
\hline & 96 & 8.755 & $1.24 \mathrm{E}+13$ & $1.23 \mathrm{E}+13$ & 9.94E-01 & $9.88 \mathrm{E}-01$ & $2.86 \mathrm{E}-02$ \\
\hline & 122 & 7.954 & $8.32 \mathrm{E}+10$ & $8.10 \mathrm{E}+10$ & $3.94 \mathrm{E}-03$ & $3.84 \mathrm{E}-03$ & $1.03 \mathrm{E}-04$ \\
\hline & 124 & 7.948 & $5.92 \mathrm{E}+10$ & $6.00 \mathrm{E}+10$ & $2.80 \mathrm{E}-03$ & $2.84 \mathrm{E}-03$ & 7.33E-05 \\
\hline & 129 & 7.944 & $4.58 \mathrm{E}+11$ & $4.29 \mathrm{E}+11$ & 3.03E-02 & $2.84 \mathrm{E}-02$ & 7.93E-04 \\
\hline & 131 & 7.939 & $1.05 \mathrm{E}+09$ & $9.36 \mathrm{E}+08$ & $6.94 \mathrm{E}-05$ & 6.19E-05 & $1.81 \mathrm{E}-06$ \\
\hline & 141 & 7.873 & $5.06 \mathrm{E}+11$ & $4.75 E+11$ & $2.35 \mathrm{E}-02$ & $2.21 \mathrm{E}-02$ & $6.09 \mathrm{E}-04$ \\
\hline & 143 & 7.869 & $2.28 \mathrm{E}+11$ & $2.34 \mathrm{E}+11$ & $1.64 \mathrm{E}-04$ & $1.69 \mathrm{E}-04$ & $6.52 \mathrm{E}-03$ \\
\hline & 146 & 7.867 & $1.32 \mathrm{E}+11$ & $1.23 \mathrm{E}+11$ & $8.58 \mathrm{E}-03$ & $7.98 \mathrm{E}-03$ & $2.22 \mathrm{E}-04$ \\
\hline & 157 & 7.860 & $5.00 \mathrm{E}+09$ & $4.59 \mathrm{E}+09$ & $3.24 \mathrm{E}-04$ & $2.97 \mathrm{E}-04$ & 8.39E-06 \\
\hline & 159 & 7.858 & $6.05 \mathrm{E}+12$ & $5.61 \mathrm{E}+12$ & 3.92E-01 & $3.64 \mathrm{E}-01$ & $1.01 \mathrm{E}-02$ \\
\hline & 161 & 7.858 & $4.52 \mathrm{E}+11$ & $4.19 \mathrm{E}+11$ & 2.93E-02 & $2.72 \mathrm{E}-02$ & 7.58E-04 \\
\hline \multirow[t]{14}{*}{10} & 22 & 12.614 & $2.80 \mathrm{E}+10$ & $2.56 \mathrm{E}+10$ & $2.00 \mathrm{E}-03$ & $1.83 \mathrm{E}-03$ & $8.32 \mathrm{E}-05$ \\
\hline & 25 & 12.371 & $9.36 \mathrm{E}+11$ & $8.98 \mathrm{E}+11$ & $6.44 \mathrm{E}-02$ & $6.18 \mathrm{E}-02$ & $2.62 \mathrm{E}-03$ \\
\hline & 36 & 12.168 & $3.47 \mathrm{E}+11$ & $3.35 \mathrm{E}+11$ & $2.31 \mathrm{E}-02$ & 2.23E-02 & $9.27 \mathrm{E}-04$ \\
\hline & 41 & 11.999 & $1.75 \mathrm{E}+11$ & $1.75 \mathrm{E}+11$ & $1.13 \mathrm{E}-02$ & $1.13 \mathrm{E}-02$ & 4.47E-04 \\
\hline & 46 & 11.898 & $2.00 \mathrm{E}+13$ & $2.00 \mathrm{E}+13$ & $1.27 \mathrm{E}+00$ & $1.27 \mathrm{E}+00$ & 4.99E-02 \\
\hline & 49 & 9.447 & $2.36 \mathrm{E}+10$ & $2.15 E+10$ & $9.48 \mathrm{E}-04$ & 8.63E-04 & $2.95 \mathrm{E}-05$ \\
\hline & 52 & 9.432 & $1.19 \mathrm{E}+11$ & $8.98 \mathrm{E}+10$ & 4.76E-03 & $3.59 \mathrm{E}-03$ & $1.48 \mathrm{E}-04$ \\
\hline & 75 & 9.038 & $4.16 \mathrm{E}+11$ & $2.88 \mathrm{E}+11$ & $1.53 \mathrm{E}-02$ & $1.06 \mathrm{E}-02$ & $4.55 \mathrm{E}-04$ \\
\hline & 84 & 8.950 & $8.92 \mathrm{E}+10$ & $8.83 \mathrm{E}+10$ & $3.21 \mathrm{E}-03$ & $3.18 \mathrm{E}-03$ & $9.47 \mathrm{E}-05$ \\
\hline & 95 & 8.929 & $6.60 \mathrm{E}+12$ & $6.72 \mathrm{E}+12$ & 2.37E-01 & $2.41 \mathrm{E}-01$ & $6.96 \mathrm{E}-03$ \\
\hline & 125 & 8.089 & $4.44 \mathrm{E}+11$ & $3.51 \mathrm{E}+11$ & $1.31 \mathrm{E}-02$ & $1.03 \mathrm{E}-02$ & $3.48 \mathrm{E}-04$ \\
\hline & 143 & 8.008 & $4.98 \mathrm{E}+10$ & $4.43 \mathrm{E}+10$ & $1.44 \mathrm{E}-03$ & $1.28 \mathrm{E}-03$ & $3.79 \mathrm{E}-05$ \\
\hline & 152 & 8.001 & $3.34 \mathrm{E}+12$ & $3.01 \mathrm{E}+12$ & $9.62 \mathrm{E}-02$ & $8.66 \mathrm{E}-02$ & $2.53 \mathrm{E}-03$ \\
\hline & 170 & 5.933 & $3.41 \mathrm{E}+09$ & $3.70 \mathrm{E}+09$ & $5.40 \mathrm{E}-05$ & $5.86 \mathrm{E}-05$ & $1.05 \mathrm{E}-06$ \\
\hline \multirow[t]{7}{*}{11} & 29 & 148.179 & $4.55 \mathrm{E}+06$ & $4.05 \mathrm{E}+06$ & 7.49E-05 & $6.66 \mathrm{E}-05$ & $3.66 \mathrm{E}-05$ \\
\hline & 33 & 136.923 & $1.38 \mathrm{E}+08$ & $1.16 \mathrm{E}+08$ & $1.94 \mathrm{E}-03$ & $1.63 \mathrm{E}-03$ & $8.72 \mathrm{E}-04$ \\
\hline & 36 & 133.619 & $2.29 \mathrm{E}+07$ & $2.47 \mathrm{E}+07$ & $1.98 \mathrm{E}-04$ & $2.14 \mathrm{E}-04$ & $8.73 \mathrm{E}-05$ \\
\hline & 38 & 121.958 & $2.96 \mathrm{E}+07$ & $2.48 \mathrm{E}+07$ & $3.30 \mathrm{E}-04$ & $2.76 \mathrm{E}-04$ & $1.33 \mathrm{E}-04$ \\
\hline & 41 & 115.671 & $9.21 \mathrm{E}+07$ & $9.34 \mathrm{E}+07$ & $5.62 \mathrm{E}-04$ & $5.70 \mathrm{E}-04$ & $2.14 \mathrm{E}-04$ \\
\hline & 42 & 115.648 & $1.23 \mathrm{E}+08$ & $1.16 \mathrm{E}+08$ & $1.23 \mathrm{E}-03$ & $1.16 \mathrm{E}-03$ & 4.70E-04 \\
\hline & 43 & 114.161 & $9.55 \mathrm{E}+07$ & $9.61 \mathrm{E}+07$ & $1.87 \mathrm{E}-04$ & $1.88 \mathrm{E}-04$ & 7.02E-05 \\
\hline
\end{tabular}


Table 6. Cont.

\begin{tabular}{|c|c|c|c|c|c|c|c|}
\hline LL. & UL. & $\lambda$ & $A_{L}$ & $A_{V}$ & $f_{L}$ & $f_{V}$ & $S_{L}$ \\
\hline & 46 & 106.935 & $1.50 \mathrm{E}+07$ & $1.74 \mathrm{E}+07$ & $8.96 \mathrm{E}-05$ & $1.04 \mathrm{E}-04$ & $3.16 \mathrm{E}-05$ \\
\hline & 49 & 32.101 & $7.52 \mathrm{E}+11$ & $7.13 E+11$ & 3.30E-01 & $3.13 \mathrm{E}-01$ & $3.49 \mathrm{E}-02$ \\
\hline & 51 & 31.950 & $8.52 \mathrm{E}+11$ & $8.97 \mathrm{E}+11$ & $6.52 \mathrm{E}-01$ & $6.87 \mathrm{E}-01$ & $6.86 \mathrm{E}-02$ \\
\hline & 52 & 31.920 & $1.65 \mathrm{E}+11$ & $1.56 \mathrm{E}+11$ & 7.14E-02 & $6.75 \mathrm{E}-02$ & $7.50 \mathrm{E}-03$ \\
\hline & 57 & 31.310 & $6.52 \mathrm{E}+05$ & $6.66 \mathrm{E}+05$ & 4.79E-07 & 4.89E-07 & 4.94E-08 \\
\hline & 69 & 27.913 & $2.81 \mathrm{E}+09$ & $3.18 \mathrm{E}+09$ & $1.12 \mathrm{E}-03$ & $1.27 \mathrm{E}-03$ & $1.03 \mathrm{E}-04$ \\
\hline & 81 & 27.110 & $4.73 \mathrm{E}+08$ & $4.31 \mathrm{E}+08$ & 2.61E-04 & $2.38 \mathrm{E}-04$ & $2.33 \mathrm{E}-05$ \\
\hline & 84 & 27.001 & $9.31 \mathrm{E}+09$ & $9.74 \mathrm{E}+09$ & $3.19 \mathrm{E}-03$ & $3.34 \mathrm{E}-03$ & 2.84E-04 \\
\hline & 85 & 26.992 & $7.18 \mathrm{E}+09$ & $6.62 \mathrm{E}+09$ & $3.92 \mathrm{E}-03$ & $3.62 \mathrm{E}-03$ & $3.48 \mathrm{E}-04$ \\
\hline & 88 & 26.947 & $1.29 \mathrm{E}+10$ & $1.31 \mathrm{E}+10$ & $1.40 \mathrm{E}-03$ & $1.43 \mathrm{E}-03$ & $1.24 \mathrm{E}-04$ \\
\hline & 95 & 26.814 & $1.23 \mathrm{E}+09$ & $1.33 \mathrm{E}+09$ & 4.31E-04 & $4.66 \mathrm{E}-04$ & $3.80 \mathrm{E}-05$ \\
\hline & 99 & 22.407 & $4.38 \mathrm{E}+11$ & $4.27 \mathrm{E}+11$ & $9.64 \mathrm{E}-02$ & $9.40 \mathrm{E}-02$ & $7.11 \mathrm{E}-03$ \\
\hline & 100 & 22.379 & $5.56 \mathrm{E}+11$ & $5.32 \mathrm{E}+11$ & 4.17E-02 & $4.00 \mathrm{E}-02$ & $3.08 \mathrm{E}-03$ \\
\hline & 101 & 22.366 & $4.95 \mathrm{E}+11$ & $5.08 \mathrm{E}+11$ & $1.85 \mathrm{E}-01$ & $1.91 \mathrm{E}-01$ & $1.37 \mathrm{E}-02$ \\
\hline & 102 & 22.356 & $7.52 \mathrm{E}+10$ & $7.33 \mathrm{E}+10$ & $1.65 \mathrm{E}-02$ & $1.61 \mathrm{E}-02$ & $1.21 \mathrm{E}-03$ \\
\hline & 110 & 22.211 & $3.03 \mathrm{E}+05$ & $3.09 \mathrm{E}+05$ & $1.12 \mathrm{E}-07$ & $1.14 \mathrm{E}-07$ & $8.20 \mathrm{E}-09$ \\
\hline & 116 & 20.695 & $1.55 \mathrm{E}+08$ & $1.97 \mathrm{E}+08$ & $3.80 \mathrm{E}-05$ & $4.83 \mathrm{E}-05$ & $2.59 \mathrm{E}-06$ \\
\hline & 122 & 20.486 & $2.27 \mathrm{E}+08$ & $2.29 \mathrm{E}+08$ & $7.15 \mathrm{E}-05$ & 7.21E-05 & $4.82 \mathrm{E}-06$ \\
\hline & 124 & 20.447 & $4.28 \mathrm{E}+09$ & $4.34 \mathrm{E}+09$ & $1.34 \mathrm{E}-03$ & $1.36 \mathrm{E}-03$ & $9.02 \mathrm{E}-05$ \\
\hline & 125 & 20.437 & $1.74 \mathrm{E}+09$ & $1.75 E+09$ & $3.28 \mathrm{E}-04$ & $3.30 \mathrm{E}-04$ & $2.21 \mathrm{E}-05$ \\
\hline & 134 & 20.153 & $2.70 \mathrm{E}+07$ & $2.85 \mathrm{E}+07$ & $5.21 \mathrm{E}-06$ & $5.50 \mathrm{E}-06$ & $3.46 \mathrm{E}-07$ \\
\hline & 141 & 19.956 & $1.77 \mathrm{E}+08$ & $1.77 \mathrm{E}+08$ & $5.30 \mathrm{E}-05$ & $5.29 \mathrm{E}-05$ & $3.48 \mathrm{E}-06$ \\
\hline & 143 & 19.932 & $5.36 \mathrm{E}+09$ & $5.40 \mathrm{E}+09$ & $9.64 \mathrm{E}-04$ & $9.71 \mathrm{E}-04$ & $6.33 \mathrm{E}-05$ \\
\hline & 144 & 19.926 & $3.84 \mathrm{E}+09$ & $3.81 \mathrm{E}+09$ & $1.14 \mathrm{E}-03$ & $1.13 \mathrm{E}-03$ & $7.50 \mathrm{E}-05$ \\
\hline & 149 & 19.896 & $8.31 \mathrm{E}+09$ & $8.21 \mathrm{E}+09$ & 4.93E-04 & $4.87 \mathrm{E}-04$ & $3.23 \mathrm{E}-05$ \\
\hline & 152 & 19.885 & $8.13 \mathrm{E}+08$ & $8.22 \mathrm{E}+08$ & $1.46 \mathrm{E}-04$ & $1.48 \mathrm{E}-04$ & $9.57 \mathrm{E}-06$ \\
\hline & 153 & 19.884 & $3.84 \mathrm{E}+04$ & $3.92 \mathrm{E}+04$ & $1.14 \mathrm{E}-08$ & $1.16 \mathrm{E}-08$ & $7.45 \mathrm{E}-10$ \\
\hline & 167 & 10.795 & $7.85 \mathrm{E}+12$ & $1.09 \mathrm{E}+13$ & $1.37 \mathrm{E}-01$ & $1.91 \mathrm{E}-01$ & 4.87E-03 \\
\hline & 178 & 10.523 & $4.33 \mathrm{E}+07$ & $8.17 \mathrm{E}+07$ & $3.60 \mathrm{E}-06$ & $6.78 \mathrm{E}-06$ & $1.25 \mathrm{E}-07$ \\
\hline \multirow[t]{10}{*}{12} & 22 & 260.791 & 7.32E+08 & $5.94 \mathrm{E}+08$ & $2.24 \mathrm{E}-02$ & $1.82 \mathrm{E}-02$ & $1.92 \mathrm{E}-02$ \\
\hline & 25 & 185.516 & $7.49 \mathrm{E}+09$ & $7.10 \mathrm{E}+09$ & $1.16 \mathrm{E}-01$ & $1.10 \mathrm{E}-01$ & 7.08E-02 \\
\hline & 49 & 32.887 & $1.61 \mathrm{E}+11$ & $1.54 \mathrm{E}+11$ & $7.82 \mathrm{E}-02$ & $7.51 \mathrm{E}-02$ & $8.47 \mathrm{E}-03$ \\
\hline & 52 & 32.697 & $6.66 \mathrm{E}+11$ & $6.32 \mathrm{E}+11$ & $3.20 \mathrm{E}-01$ & $3.04 \mathrm{E}-01$ & $3.45 \mathrm{E}-02$ \\
\hline & 61 & 29.522 & $4.56 \mathrm{E}+08$ & $4.15 \mathrm{E}+08$ & $1.79 \mathrm{E}-04$ & $1.63 \mathrm{E}-04$ & $1.74 \mathrm{E}-05$ \\
\hline & 84 & 27.555 & $1.79 \mathrm{E}+08$ & $1.54 \mathrm{E}+08$ & $6.10 \mathrm{E}-05$ & $5.27 \mathrm{E}-05$ & $5.54 \mathrm{E}-06$ \\
\hline & 95 & 27.360 & $1.18 \mathrm{E}+10$ & $1.14 \mathrm{E}+10$ & $3.98 \mathrm{E}-03$ & $3.83 \mathrm{E}-03$ & $3.59 \mathrm{E}-04$ \\
\hline & 99 & 22.787 & $7.95 E+10$ & $6.65 E+10$ & $1.86 \mathrm{E}-02$ & $1.55 \mathrm{E}-02$ & $1.39 \mathrm{E}-03$ \\
\hline & 102 & 22.735 & $4.33 \mathrm{E}+11$ & $3.60 \mathrm{E}+11$ & $1.01 \mathrm{E}-01$ & $8.36 \mathrm{E}-02$ & $7.53 \mathrm{E}-03$ \\
\hline & 116 & 21.019 & $1.24 \mathrm{E}+08$ & $1.74 \mathrm{E}+06$ & $2.46 \mathrm{E}-05$ & $3.46 \mathrm{E}-07$ & $1.70 \mathrm{E}-06$ \\
\hline
\end{tabular}


Table 6. Cont.

\begin{tabular}{|c|c|c|c|c|c|c|c|}
\hline LL. & UL. & $\lambda$ & $A_{L}$ & $A_{V}$ & $f_{L}$ & $f_{V}$ & $S_{L}$ \\
\hline & 125 & 20.753 & $5.65 \mathrm{E}+09$ & $4.28 \mathrm{E}+09$ & $1.09 \mathrm{E}-03$ & 8.29E-04 & 7.47E-05 \\
\hline & 143 & 20.232 & $1.00 \mathrm{E}+08$ & $8.71 \mathrm{E}+07$ & $1.84 \mathrm{E}-05$ & $1.60 \mathrm{E}-05$ & $1.23 \mathrm{E}-06$ \\
\hline & 152 & 20.184 & $6.64 \mathrm{E}+09$ & $6.08 \mathrm{E}+09$ & $1.22 \mathrm{E}-03$ & $1.11 \mathrm{E}-03$ & 8.09E-05 \\
\hline & 186 & 10.880 & $1.25 \mathrm{E}+11$ & $1.49 \mathrm{E}+11$ & $6.65 \mathrm{E}-03$ & $7.93 \mathrm{E}-03$ & $2.38 \mathrm{E}-04$ \\
\hline & 170 & 10.741 & $5.17 \mathrm{E}+12$ & $6.28 \mathrm{E}+12$ & $2.68 \mathrm{E}-01$ & $3.26 \mathrm{E}-01$ & $9.48 \mathrm{E}-03$ \\
\hline \multirow[t]{23}{*}{13} & 35 & 174.866 & $1.86 \mathrm{E}+09$ & $2.90 \mathrm{E}+09$ & $2.45 \mathrm{E}-02$ & $3.83 \mathrm{E}-02$ & $6.65 \mathrm{E}-02$ \\
\hline & 37 & 157.412 & $1.06 \mathrm{E}+08$ & $1.32 \mathrm{E}+08$ & $1.02 \mathrm{E}-03$ & $1.27 \mathrm{E}-03$ & $2.45 \mathrm{E}-03$ \\
\hline & 47 & 34.693 & $1.42 \mathrm{E}+11$ & $1.49 \mathrm{E}+11$ & 8.05E-02 & $8.45 \mathrm{E}-02$ & $9.20 \mathrm{E}-03$ \\
\hline & 48 & 34.352 & $2.66 \mathrm{E}+11$ & $2.36 \mathrm{E}+11$ & 4.71E-02 & $4.18 \mathrm{E}-02$ & $5.33 \mathrm{E}-03$ \\
\hline & 53 & 33.348 & $1.10 \mathrm{E}+12$ & $1.06 \mathrm{E}+12$ & $1.00 \mathrm{E}-01$ & $9.72 \mathrm{E}-02$ & $8.85 \mathrm{E}-01$ \\
\hline & 54 & 33.333 & $5.26 \mathrm{E}+11$ & $5.11 \mathrm{E}+11$ & $2.55 \mathrm{E}-01$ & $2.48 \mathrm{E}-01$ & $2.80 \mathrm{E}-02$ \\
\hline & 56 & 33.083 & $5.70 \mathrm{E}+11$ & $5.57 \mathrm{E}+11$ & $5.10 \mathrm{E}-02$ & $4.98 \mathrm{E}-02$ & 4.57E-01 \\
\hline & 64 & 32.516 & $7.99 \mathrm{E}+11$ & $8.06 \mathrm{E}+11$ & $6.78 \mathrm{E}-02$ & $6.84 \mathrm{E}-02$ & 6.39E-01 \\
\hline & 72 & 29.218 & $1.37 \mathrm{E}+10$ & $1.36 \mathrm{E}+10$ & $8.41 \mathrm{E}-04$ & $8.36 \mathrm{E}-04$ & $8.69 \mathrm{E}-03$ \\
\hline & 79 & 28.758 & $9.08 \mathrm{E}+09$ & $9.87 \mathrm{E}+09$ & $3.67 \mathrm{E}-03$ & 3.99E-03 & $3.48 \mathrm{E}-04$ \\
\hline & 82 & 28.299 & $1.38 \mathrm{E}+10$ & $1.20 \mathrm{E}+10$ & $1.66 \mathrm{E}-03$ & $1.44 \mathrm{E}-03$ & $1.55 \mathrm{E}-04$ \\
\hline & 89 & 28.173 & $9.17 \mathrm{E}+09$ & $9.82 \mathrm{E}+09$ & $5.06 \mathrm{E}-04$ & $5.42 \mathrm{E}-04$ & $5.84 \mathrm{E}-03$ \\
\hline & 97 & 28.091 & $5.51 \mathrm{E}+09$ & $5.80 \mathrm{E}+09$ & $2.06 \mathrm{E}-03$ & $2.17 \mathrm{E}-03$ & $1.90 \mathrm{E}-04$ \\
\hline & 103 & 23.144 & $5.54 \mathrm{E}+11$ & $5.74 \mathrm{E}+11$ & $1.70 \mathrm{E}-02$ & $1.75 \mathrm{E}-02$ & $2.30 \mathrm{E}-01$ \\
\hline & 104 & 23.448 & $6.50 \mathrm{E}+10$ & $8.60 \mathrm{E}+10$ & $2.13 \mathrm{E}-02$ & $2.82 \mathrm{E}-02$ & $1.64 \mathrm{E}-03$ \\
\hline & 105 & 23.085 & $2.90 \mathrm{E}+11$ & $3.03 E+11$ & $8.80 \mathrm{E}-03$ & $9.20 \mathrm{E}-03$ & $1.21 \mathrm{E}-01$ \\
\hline & 118 & 21.322 & $1.17 \mathrm{E}+09$ & $1.20 \mathrm{E}+09$ & $2.45 \mathrm{E}-04$ & $2.51 \mathrm{E}-04$ & $1.72 \mathrm{E}-05$ \\
\hline & 127 & 21.143 & $5.81 \mathrm{E}+09$ & $6.55 \mathrm{E}+09$ & $1.35 \mathrm{E}-04$ & $1.53 \mathrm{E}-04$ & $2.20 \mathrm{E}-03$ \\
\hline & 136 & 20.738 & $1.77 \mathrm{E}+09$ & $1.77 \mathrm{E}+09$ & $3.89 \mathrm{E}-05$ & 3.89E-05 & $5.69 \mathrm{E}-04$ \\
\hline & 147 & 20.590 & $3.27 \mathrm{E}+09$ & $3.96 \mathrm{E}+09$ & $7.04 \mathrm{E}-05$ & $8.52 \mathrm{E}-05$ & $1.26 \mathrm{E}-03$ \\
\hline & 151 & 20.567 & $2.16 \mathrm{E}+06$ & $2.71 \mathrm{E}+05$ & 4.64E-08 & 5.81E-09 & $8.58 \mathrm{E}-08$ \\
\hline & 154 & 20.562 & $2.16 \mathrm{E}+09$ & $2.58 \mathrm{E}+09$ & 4.91E-04 & $5.86 \mathrm{E}-04$ & $3.32 \mathrm{E}-05$ \\
\hline & 176 & 10.770 & $2.10 \mathrm{E}+12$ & $2.11 \mathrm{E}+12$ & $1.10 \mathrm{E}-01$ & $1.11 \mathrm{E}-01$ & $3.90 \mathrm{E}-03$ \\
\hline \multirow[t]{6}{*}{14} & 47 & 34.812 & $7.08 \mathrm{E}+10$ & $8.45 \mathrm{E}+10$ & $3.86 \mathrm{E}-02$ & 4.61E-02 & 4.42E-03 \\
\hline & 54 & 33.442 & $1.14 \mathrm{E}+12$ & $1.13 \mathrm{E}+12$ & $5.72 \mathrm{E}-01$ & $5.66 \mathrm{E}-01$ & $6.30 \mathrm{E}-02$ \\
\hline & 97 & 28.169 & $1.14 \mathrm{E}+10$ & $1.19 \mathrm{E}+10$ & $4.08 \mathrm{E}-03$ & $4.23 \mathrm{E}-03$ & $3.78 \mathrm{E}-04$ \\
\hline & 106 & 23.184 & $5.71 \mathrm{E}+11$ & $6.13 E+11$ & $1.38 \mathrm{E}-01$ & $1.48 \mathrm{E}-01$ & $1.05 \mathrm{E}-02$ \\
\hline & 118 & 21.366 & $7.47 \mathrm{E}+08$ & $7.40 \mathrm{E}+07$ & $1.53 \mathrm{E}-04$ & $1.52 \mathrm{E}-05$ & $1.08 \mathrm{E}-05$ \\
\hline & 176 & 10.782 & $4.56 \mathrm{E}+12$ & $4.68 \mathrm{E}+12$ & 2.39E-01 & $2.45 \mathrm{E}-01$ & $8.47 \mathrm{E}-03$ \\
\hline \multirow[t]{4}{*}{15} & 27 & 236.890 & $1.51 \mathrm{E}+09$ & $1.51 \mathrm{E}+09$ & $3.81 \mathrm{E}-02$ & $3.81 \mathrm{E}-02$ & 2.97E-02 \\
\hline & 34 & 185.547 & $2.42 \mathrm{E}+09$ & $2.72 \mathrm{E}+09$ & $4.22 \mathrm{E}-02$ & 4.74E-02 & $2.58 \mathrm{E}-02$ \\
\hline & 35 & 185.128 & $5.30 \mathrm{E}+08$ & $4.59 \mathrm{E}+08$ & 8.30E-03 & $7.18 \mathrm{E}-03$ & $1.18 \mathrm{E}-02$ \\
\hline & 47 & 35.079 & $9.64 \mathrm{E}+10$ & $9.82 \mathrm{E}+10$ & $5.43 \mathrm{E}-02$ & $5.53 \mathrm{E}-02$ & $6.28 \mathrm{E}-03$ \\
\hline
\end{tabular}


Table 6. Cont.

\begin{tabular}{|c|c|c|c|c|c|c|c|}
\hline LL. & UL. & $\lambda$ & $A_{L}$ & $A_{V}$ & $f_{L}$ & $f_{V}$ & $S_{L}$ \\
\hline & 48 & 34.730 & $4.31 \mathrm{E}+11$ & $3.84 \mathrm{E}+11$ & 7.79E-02 & $6.94 \mathrm{E}-02$ & $8.90 \mathrm{E}-03$ \\
\hline & 53 & 33.705 & $4.53 \mathrm{E}+11$ & $4.37 \mathrm{E}+11$ & $4.28 \mathrm{E}-02$ & 4.13E-02 & $3.72 \mathrm{E}-01$ \\
\hline & 56 & 33.434 & $1.27 \mathrm{E}+12$ & $1.24 \mathrm{E}+12$ & $1.17 \mathrm{E}-01$ & $1.15 \mathrm{E}-01$ & $1.04 \mathrm{E}+00$ \\
\hline & 65 & 30.228 & $1.80 \mathrm{E}+10$ & $1.56 \mathrm{E}+10$ & $6.42 \mathrm{E}-03$ & $5.56 \mathrm{E}-03$ & $6.39 \mathrm{E}-04$ \\
\hline & 72 & 29.491 & $1.67 \mathrm{E}+09$ & $1.73 \mathrm{E}+09$ & $1.06 \mathrm{E}-04$ & $1.10 \mathrm{E}-04$ & $1.13 \mathrm{E}-03$ \\
\hline & 76 & 29.022 & $9.43 \mathrm{E}+09$ & $5.08 \mathrm{E}+09$ & 1.19E-03 & $7.16 \mathrm{E}-04$ & $3.41 \mathrm{E}-04$ \\
\hline & 80 & 28.765 & $6.61 \mathrm{E}+09$ & $8.05 E+09$ & $3.88 \mathrm{E}-04$ & 4.73E-04 & $4.99 \mathrm{E}-03$ \\
\hline & 82 & 28.555 & $1.27 \mathrm{E}+10$ & $7.23 \mathrm{E}+09$ & $1.56 \mathrm{E}-03$ & 8.84E-04 & $1.46 \mathrm{E}-04$ \\
\hline & 93 & 28.343 & $3.17 \mathrm{E}+09$ & $3.35 \mathrm{E}+09$ & $1.21 \mathrm{E}-03$ & $1.28 \mathrm{E}-03$ & $1.13 \mathrm{E}-04$ \\
\hline & 94 & 28.336 & $1.70 \mathrm{E}+10$ & $1.75 \mathrm{E}+10$ & $9.57 \mathrm{E}-04$ & 9.84E-04 & $1.06 \mathrm{E}-02$ \\
\hline & 97 & 23.623 & $4.32 \mathrm{E}+10$ & $5.58 \mathrm{E}+10$ & $1.40 \mathrm{E}-02$ & $1.81 \mathrm{E}-02$ & $1.09 \mathrm{E}-03$ \\
\hline & 103 & 23.316 & $2.14 \mathrm{E}+11$ & $2.20 \mathrm{E}+11$ & $6.70 \mathrm{E}-03$ & $6.88 \mathrm{E}-03$ & 8.97E-02 \\
\hline & 105 & 23.255 & $6.51 \mathrm{E}+11$ & $6.80 \mathrm{E}+11$ & 2.02E-02 & $2.11 \mathrm{E}-02$ & $2.75 \mathrm{E}-01$ \\
\hline & 118 & 21.467 & $1.21 \mathrm{E}+10$ & $9.86 \mathrm{E}+09$ & 2.04E-03 & $1.66 \mathrm{E}-03$ & $1.44 \mathrm{E}-04$ \\
\hline & 120 & 21.411 & $1.48 \mathrm{E}+10$ & $1.23 \mathrm{E}+10$ & $3.59 \mathrm{E}-04$ & 2.99E-04 & $4.24 \mathrm{E}-03$ \\
\hline & 121 & 21.402 & $6.90 \mathrm{E}+09$ & $6.15 \mathrm{E}+09$ & $1.27 \mathrm{E}-03$ & $1.13 \mathrm{E}-03$ & 8.93E-05 \\
\hline & 127 & 21.285 & $6.58 \mathrm{E}+08$ & $7.35 \mathrm{E}+08$ & $1.57 \mathrm{E}-05$ & $1.75 \mathrm{E}-05$ & $2.50 \mathrm{E}-04$ \\
\hline & 136 & 20.875 & $2.05 \mathrm{E}+09$ & $1.88 \mathrm{E}+09$ & $4.60 \mathrm{E}-05$ & $4.21 \mathrm{E}-05$ & $6.13 \mathrm{E}-04$ \\
\hline & 137 & 20.874 & $3.64 \mathrm{E}+09$ & $3.56 \mathrm{E}+09$ & $6.99 \mathrm{E}-04$ & $6.84 \mathrm{E}-04$ & $4.80 \mathrm{E}-05$ \\
\hline & 140 & 20.819 & $4.71 \mathrm{E}+09$ & $5.72 \mathrm{E}+09$ & $1.05 \mathrm{E}-04$ & $1.27 \mathrm{E}-04$ & $1.86 \mathrm{E}-03$ \\
\hline & 151 & 20.702 & $6.34 \mathrm{E}+09$ & $7.84 \mathrm{E}+09$ & 1.39E-04 & $1.72 \mathrm{E}-04$ & $2.52 \mathrm{E}-03$ \\
\hline & 154 & 20.697 & $1.27 \mathrm{E}+09$ & $1.50 \mathrm{E}+09$ & 2.88E-04 & $3.40 \mathrm{E}-04$ & $1.97 \mathrm{E}-05$ \\
\hline & 171 & 10.879 & $6.12 \mathrm{E}+12$ & $4.90 \mathrm{E}+12$ & $1.94 \mathrm{E}-02$ & $1.56 \mathrm{E}-02$ & $4.35 \mathrm{E}-01$ \\
\hline & 176 & 10.807 & $1.36 \mathrm{E}+12$ & $1.42 \mathrm{E}+12$ & 7.46E-02 & 7.79E-02 & $2.65 \mathrm{E}-03$ \\
\hline \multirow[t]{13}{*}{16} & 26 & 242.993 & $1.11 \mathrm{E}+09$ & $1.15 \mathrm{E}+09$ & 4.93E-02 & $5.11 \mathrm{E}-02$ & $3.94 \mathrm{E}-02$ \\
\hline & 47 & 35.148 & $4.21 \mathrm{E}+11$ & $4.09 \mathrm{E}+11$ & $2.34 \mathrm{E}-01$ & $2.27 \mathrm{E}-01$ & $2.71 \mathrm{E}-02$ \\
\hline & 53 & 33.769 & $5.00 \mathrm{E}+11$ & $5.12 \mathrm{E}+11$ & $4.28 \mathrm{E}-01$ & 4.38E-01 & $4.75 \mathrm{E}-02$ \\
\hline & 54 & 33.753 & $5.69 \mathrm{E}+10$ & $5.73 \mathrm{E}+10$ & $2.92 \mathrm{E}-02$ & $2.94 \mathrm{E}-02$ & $3.24 \mathrm{E}-03$ \\
\hline & 55 & 33.626 & $1.94 \mathrm{E}+12$ & $2.07 \mathrm{E}+12$ & $2.31 \mathrm{E}+00$ & $2.46 \mathrm{E}+00$ & $2.55 \mathrm{E}-01$ \\
\hline & 72 & 29.540 & $1.59 \mathrm{E}+09$ & $1.64 \mathrm{E}+09$ & $1.04 \mathrm{E}-03$ & $1.07 \mathrm{E}-03$ & $1.01 \mathrm{E}-04$ \\
\hline & 73 & 29.539 & $2.85 \mathrm{E}+08$ & $2.78 \mathrm{E}+08$ & 2.61E-04 & $2.54 \mathrm{E}-04$ & $2.54 \mathrm{E}-05$ \\
\hline & 74 & 29.501 & $1.58 \mathrm{E}+10$ & $1.54 \mathrm{E}+10$ & $1.44 \mathrm{E}-02$ & $1.40 \mathrm{E}-02$ & $1.40 \mathrm{E}-03$ \\
\hline & 79 & 29.070 & $1.00 \mathrm{E}+09$ & $1.13 \mathrm{E}+09$ & $3.81 \mathrm{E}-04$ & $4.28 \mathrm{E}-04$ & $3.64 \mathrm{E}-05$ \\
\hline & 89 & 28.472 & $2.28 \mathrm{E}+09$ & $2.11 \mathrm{E}+09$ & $1.39 \mathrm{E}-03$ & $1.28 \mathrm{E}-03$ & $1.30 \mathrm{E}-04$ \\
\hline & 91 & 28.461 & $2.11 \mathrm{E}+07$ & $1.99 \mathrm{E}+07$ & $1.79 \mathrm{E}-05$ & $1.69 \mathrm{E}-05$ & $1.68 \mathrm{E}-06$ \\
\hline & 94 & 28.381 & $2.32 \mathrm{E}+09$ & $2.27 \mathrm{E}+09$ & $1.40 \mathrm{E}-03$ & $1.37 \mathrm{E}-03$ & $1.31 \mathrm{E}-04$ \\
\hline & 97 & 23.655 & $2.42 \mathrm{E}+11$ & $1.85 \mathrm{E}+11$ & $6.09 \mathrm{E}-02$ & 4.66E-02 & $4.74 \mathrm{E}-03$ \\
\hline
\end{tabular}


Table 6. Cont.

\begin{tabular}{|c|c|c|c|c|c|c|c|}
\hline LL. & UL. & $\lambda$ & $A_{L}$ & $A_{V}$ & $f_{L}$ & $f_{V}$ & $S_{L}$ \\
\hline & 103 & 23.346 & $2.59 \mathrm{E}+11$ & $2.51 \mathrm{E}+11$ & $1.06 \mathrm{E}-01$ & $1.03 \mathrm{E}-01$ & $8.14 \mathrm{E}-03$ \\
\hline & 104 & 23.333 & $2.95 \mathrm{E}+10$ & $2.87 \mathrm{E}+10$ & 7.22E-03 & 7.03E-03 & $5.54 \mathrm{E}-04$ \\
\hline & 105 & 23.286 & $2.49 \mathrm{E}+09$ & $2.42 \mathrm{E}+09$ & $1.01 \mathrm{E}-03$ & $9.83 \mathrm{E}-04$ & $7.76 \mathrm{E}-05$ \\
\hline & 120 & 21.311 & $7.92 \mathrm{E}+08$ & $6.80 \mathrm{E}+08$ & $2.70 \mathrm{E}-04$ & $2.31 \mathrm{E}-04$ & $1.89 \mathrm{E}-05$ \\
\hline & 176 & 10.814 & $5.92 \mathrm{E}+12$ & $5.91 \mathrm{E}+12$ & $3.11 \mathrm{E}-01$ & $3.11 \mathrm{E}-01$ & $1.11 \mathrm{E}-02$ \\
\hline \multirow[t]{23}{*}{17} & 38 & 188.720 & $9.86 \mathrm{E}+08$ & $8.91 \mathrm{E}+08$ & $2.63 \mathrm{E}-02$ & $2.38 \mathrm{E}-02$ & $1.64 \mathrm{E}-02$ \\
\hline & 43 & 170.681 & $4.24 \mathrm{E}+09$ & $7.57 \mathrm{E}+09$ & $1.85 \mathrm{E}-02$ & $3.30 \mathrm{E}-02$ & $1.04 \mathrm{E}-02$ \\
\hline & 46 & 155.020 & $1.03 \mathrm{E}+08$ & $9.73 \mathrm{E}+07$ & $1.05 \mathrm{E}-03$ & 9.92E-04 & $5.37 \mathrm{E}-04$ \\
\hline & 49 & 35.397 & $2.52 \mathrm{E}+10$ & $2.92 \mathrm{E}+10$ & $1.64 \mathrm{E}-02$ & $1.90 \mathrm{E}-02$ & $1.92 \mathrm{E}-03$ \\
\hline & 51 & 35.213 & $1.38 \mathrm{E}+09$ & $1.21 \mathrm{E}+09$ & $1.28 \mathrm{E}-03$ & $1.13 \mathrm{E}-03$ & $1.49 \mathrm{E}-04$ \\
\hline & 52 & 35.177 & $5.34 \mathrm{E}+09$ & $6.07 \mathrm{E}+09$ & $3.38 \mathrm{E}-03$ & $3.84 \mathrm{E}-03$ & $3.91 \mathrm{E}-04$ \\
\hline & 57 & 34.438 & $3.33 \mathrm{E}+12$ & $3.36 \mathrm{E}+12$ & $2.96 \mathrm{E}+00$ & $2.99 \mathrm{E}+00$ & $3.35 \mathrm{E}-01$ \\
\hline & 61 & 31.529 & $1.13 \mathrm{E}+09$ & $1.03 \mathrm{E}+09$ & 4.59E-04 & 4.18E-04 & $4.76 \mathrm{E}-05$ \\
\hline & 62 & 31.515 & $4.94 \mathrm{E}+09$ & $6.40 \mathrm{E}+09$ & 7.36E-04 & $9.53 \mathrm{E}-04$ & 7.64E-05 \\
\hline & 71 & 30.315 & $6.21 \mathrm{E}+08$ & $5.38 \mathrm{E}+08$ & $4.28 \mathrm{E}-04$ & $3.70 \mathrm{E}-04$ & $4.27 \mathrm{E}-05$ \\
\hline & 75 & 30.260 & $6.53 \mathrm{E}+07$ & $6.98 \mathrm{E}+07$ & 2.87E-05 & 3.07E-05 & $2.86 \mathrm{E}-06$ \\
\hline & 85 & 29.284 & $2.55 \mathrm{E}+09$ & $1.45 \mathrm{E}+08$ & $1.64 \mathrm{E}-03$ & 9.32E-05 & $1.58 \mathrm{E}-04$ \\
\hline & 88 & 29.232 & $6.47 \mathrm{E}+08$ & $6.64 \mathrm{E}+09$ & 8.29E-05 & $8.51 \mathrm{E}-04$ & 7.97E-06 \\
\hline & 99 & 23.964 & $1.07 \mathrm{E}+10$ & $1.36 \mathrm{E}+10$ & $3.51 \mathrm{E}-03$ & $4.46 \mathrm{E}-03$ & $2.77 \mathrm{E}-04$ \\
\hline & 100 & 23.932 & $5.41 \mathrm{E}+10$ & $7.07 \mathrm{E}+10$ & 4.64E-03 & $6.07 \mathrm{E}-03$ & $3.66 \mathrm{E}-04$ \\
\hline & 102 & 23.907 & $1.72 \mathrm{E}+09$ & $2.24 \mathrm{E}+09$ & 5.76E-04 & $7.50 \mathrm{E}-04$ & 4.54E-05 \\
\hline & 110 & 23.741 & $1.15 \mathrm{E}+12$ & $1.15 \mathrm{E}+12$ & 4.86E-01 & 4.87E-01 & $3.80 \mathrm{E}-02$ \\
\hline & 116 & 22.017 & $6.70 \mathrm{E}+08$ & $7.10 \mathrm{E}+08$ & $1.55 \mathrm{E}-04$ & $1.64 \mathrm{E}-04$ & $1.12 \mathrm{E}-05$ \\
\hline & 122 & 21.780 & $1.39 \mathrm{E}+10$ & $1.49 \mathrm{E}+10$ & $4.95 \mathrm{E}-03$ & $5.28 \mathrm{E}-03$ & $3.55 \mathrm{E}-04$ \\
\hline & 124 & 21.737 & $1.23 \mathrm{E}+09$ & $1.34 \mathrm{E}+09$ & $4.36 \mathrm{E}-04$ & $4.75 \mathrm{E}-04$ & $3.12 \mathrm{E}-05$ \\
\hline & 134 & 21.419 & $2.88 \mathrm{E}+07$ & $3.36 \mathrm{E}+07$ & $9.90 \mathrm{E}-06$ & $1.16 \mathrm{E}-05$ & $6.98 \mathrm{E}-07$ \\
\hline & 141 & 21.183 & $1.22 \mathrm{E}+09$ & $9.18 \mathrm{E}+08$ & 4.11E-04 & 3.09E-04 & $2.87 \mathrm{E}-05$ \\
\hline & 180 & 10.854 & $7.33 \mathrm{E}+12$ & $6.92 \mathrm{E}+12$ & $6.47 \mathrm{E}-01$ & $6.11 \mathrm{E}-01$ & $2.31 \mathrm{E}-02$ \\
\hline \multirow[t]{11}{*}{18} & 31 & 231.709 & $4.18 \mathrm{E}+08$ & $4.42 \mathrm{E}+08$ & $2.35 \mathrm{E}-02$ & 2.49E-02 & $1.80 \mathrm{E}-02$ \\
\hline & 33 & 227.876 & $1.15 \mathrm{E}+08$ & $1.27 \mathrm{E}+08$ & 4.49E-03 & 4.93E-03 & $3.37 \mathrm{E}-03$ \\
\hline & 46 & 155.365 & $6.45 \mathrm{E}+08$ & $8.60 \mathrm{E}+08$ & $3.58 \mathrm{E}-03$ & 4.77E-03 & $9.34 \mathrm{E}-03$ \\
\hline & 49 & 35.415 & $8.14 \mathrm{E}+10$ & $9.55 E+10$ & $5.35 \mathrm{E}-03$ & $6.28 \mathrm{E}-03$ & $5.39 \mathrm{E}-02$ \\
\hline & 51 & 35.231 & $2.01 \mathrm{E}+10$ & $1.75 E+10$ & $1.87 \mathrm{E}-02$ & $1.63 \mathrm{E}-02$ & $2.17 \mathrm{E}-03$ \\
\hline & 52 & 35.195 & $1.19 \mathrm{E}+10$ & $1.22 \mathrm{E}+10$ & 7.67E-04 & $7.90 \mathrm{E}-04$ & $6.82 \mathrm{E}-03$ \\
\hline & 57 & 34.455 & $6.19 \mathrm{E}+11$ & $6.22 \mathrm{E}+11$ & $5.50 \mathrm{E}-01$ & $5.54 \mathrm{E}-01$ & $6.24 \mathrm{E}-02$ \\
\hline & 58 & 34.386 & $3.53 \mathrm{E}+12$ & $3.53 E+12$ & $4.38 \mathrm{E}+00$ & $4.38 \mathrm{E}+00$ & $4.96 \mathrm{E}-01$ \\
\hline & 59 & 34.313 & $4.58 \mathrm{E}+10$ & $4.60 \mathrm{E}+10$ & $5.66 \mathrm{E}-02$ & 5.69E-02 & $6.40 \mathrm{E}-03$ \\
\hline & 68 & 30.386 & $1.13 \mathrm{E}+10$ & $1.20 \mathrm{E}+10$ & $1.10 \mathrm{E}-02$ & $1.16 \mathrm{E}-02$ & $1.10 \mathrm{E}-03$ \\
\hline & 96 & 29.078 & $1.79 \mathrm{E}+09$ & $2.23 \mathrm{E}+09$ & $1.59 \mathrm{E}-03$ & $1.98 \mathrm{E}-03$ & $1.52 \mathrm{E}-04$ \\
\hline
\end{tabular}


Table 6. Cont.

\begin{tabular}{|c|c|c|c|c|c|c|c|}
\hline LL. & UL. & $\lambda$ & $A_{L}$ & $A_{V}$ & $f_{L}$ & $f_{V}$ & $S_{L}$ \\
\hline & 102 & 23.915 & $3.77 \mathrm{E}+09$ & $3.55 \mathrm{E}+09$ & 7.63E-05 & $7.20 \mathrm{E}-05$ & $9.14 \mathrm{E}-04$ \\
\hline & 110 & 23.749 & $2.14 \mathrm{E}+11$ & $2.13 E+11$ & $9.06 \mathrm{E}-02$ & $9.01 \mathrm{E}-02$ & $7.08 \mathrm{E}-03$ \\
\hline & 111 & 23.716 & $1.25 \mathrm{E}+12$ & $1.24 \mathrm{E}+12$ & $7.36 \mathrm{E}-01$ & 7.34E-01 & $5.75 \mathrm{E}-02$ \\
\hline & 112 & 23.695 & $3.59 \mathrm{E}+09$ & $3.70 \mathrm{E}+09$ & $2.11 \mathrm{E}-03$ & $2.18 \mathrm{E}-03$ & $1.65 \mathrm{E}-04$ \\
\hline & 116 & 22.023 & $1.62 \mathrm{E}+09$ & $1.45 \mathrm{E}+09$ & $2.56 \mathrm{E}-05$ & $2.29 \mathrm{E}-05$ & $3.16 \mathrm{E}-04$ \\
\hline & 129 & 21.709 & $7.29 \mathrm{E}+09$ & $7.90 \mathrm{E}+09$ & $3.61 \mathrm{E}-03$ & $3.91 \mathrm{E}-03$ & $2.58 \mathrm{E}-04$ \\
\hline & 134 & 21.425 & $3.32 \mathrm{E}+08$ & $3.67 \mathrm{E}+08$ & $1.14 \mathrm{E}-04$ & $1.26 \mathrm{E}-04$ & $8.06 \mathrm{E}-06$ \\
\hline & 135 & 21.411 & $6.16 \mathrm{E}+08$ & $3.21 \mathrm{E}+08$ & $8.96 \mathrm{E}-06$ & $4.66 \mathrm{E}-06$ & $6.62 \mathrm{E}-05$ \\
\hline & 141 & 21.189 & $1.66 \mathrm{E}+09$ & $1.97 \mathrm{E}+09$ & $5.57 \mathrm{E}-04$ & $6.63 \mathrm{E}-04$ & $3.89 \mathrm{E}-05$ \\
\hline & 143 & 21.162 & $4.51 \mathrm{E}+08$ & $8.18 \mathrm{E}+09$ & $6.33 \mathrm{E}-06$ & $1.15 \mathrm{E}-04$ & $1.65 \mathrm{E}-03$ \\
\hline & 179 & 10.823 & $8.35 \mathrm{E}+12$ & $8.07 \mathrm{E}+12$ & $1.03 \mathrm{E}+00$ & 9.92E-01 & $3.66 \mathrm{E}-02$ \\
\hline \multirow[t]{14}{*}{19} & 24 & 388.614 & $4.13 \mathrm{E}+07$ & $3.54 \mathrm{E}+07$ & 4.67E-03 & $4.00 \mathrm{E}-03$ & $5.98 \mathrm{E}-03$ \\
\hline & 29 & 273.450 & $2.36 \mathrm{E}+04$ & $3.26 \mathrm{E}+05$ & 1.32E-06 & $1.83 \mathrm{E}-05$ & $1.19 \mathrm{E}-06$ \\
\hline & 45 & 160.530 & $9.31 \mathrm{E}+08$ & $7.58 \mathrm{E}+08$ & $2.52 \mathrm{E}-02$ & $2.05 \mathrm{E}-02$ & $1.33 \mathrm{E}-02$ \\
\hline & 57 & 34.665 & $1.78 \mathrm{E}+10$ & $1.78 \mathrm{E}+10$ & $1.61 \mathrm{E}-02$ & $1.60 \mathrm{E}-02$ & $1.83 \mathrm{E}-03$ \\
\hline & 58 & 34.597 & $4.27 \mathrm{E}+11$ & $4.30 \mathrm{E}+11$ & 5.37E-01 & $5.40 \mathrm{E}-01$ & $6.11 \mathrm{E}-02$ \\
\hline & 59 & 34.522 & $1.54 \mathrm{E}+10$ & $1.52 \mathrm{E}+10$ & $1.92 \mathrm{E}-02$ & $1.90 \mathrm{E}-02$ & $2.19 \mathrm{E}-03$ \\
\hline & 66 & 30.792 & $7.09 \mathrm{E}+06$ & $9.13 \mathrm{E}+06$ & $5.04 \mathrm{E}-06$ & $6.49 \mathrm{E}-06$ & $5.11 \mathrm{E}-07$ \\
\hline & 110 & 23.849 & $6.17 \mathrm{E}+09$ & $6.04 \mathrm{E}+09$ & 2.63E-03 & $2.57 \mathrm{E}-03$ & $2.07 \mathrm{E}-04$ \\
\hline & 111 & 23.816 & $1.53 \mathrm{E}+11$ & $1.52 \mathrm{E}+11$ & $9.13 \mathrm{E}-02$ & $9.05 \mathrm{E}-02$ & $7.16 \mathrm{E}-03$ \\
\hline & 112 & 23.794 & $2.59 \mathrm{E}+09$ & $2.47 \mathrm{E}+09$ & $1.54 \mathrm{E}-03$ & $1.47 \mathrm{E}-03$ & $1.20 \mathrm{E}-04$ \\
\hline & 134 & 21.507 & $1.60 \mathrm{E}+09$ & $1.86 \mathrm{E}+09$ & $5.55 \mathrm{E}-04$ & $6.46 \mathrm{E}-04$ & $3.93 \mathrm{E}-05$ \\
\hline & 169 & 11.132 & $2.12 \mathrm{E}+11$ & $2.20 \mathrm{E}+11$ & $1.97 \mathrm{E}-02$ & $2.04 \mathrm{E}-02$ & 7.23E-04 \\
\hline & 178 & 10.877 & $1.34 \mathrm{E}+10$ & $1.34 \mathrm{E}+10$ & $1.19 \mathrm{E}-03$ & $1.19 \mathrm{E}-03$ & $4.27 \mathrm{E}-05$ \\
\hline & 179 & 10.844 & $1.92 \mathrm{E}+12$ & $1.91 \mathrm{E}+12$ & $2.36 \mathrm{E}-01$ & $2.36 \mathrm{E}-01$ & $8.44 \mathrm{E}-03$ \\
\hline \multirow[t]{14}{*}{20} & 31 & 277.509 & $4.10 \mathrm{E}+07$ & $3.31 \mathrm{E}+07$ & 3.32E-03 & 2.67E-03 & 3.03E-03 \\
\hline & 42 & 199.217 & $1.42 \mathrm{E}+08$ & $1.24 \mathrm{E}+08$ & $4.23 \mathrm{E}-03$ & $3.69 \mathrm{E}-03$ & $2.77 \mathrm{E}-03$ \\
\hline & 45 & 175.634 & $2.27 \mathrm{E}+09$ & $1.91 \mathrm{E}+09$ & 7.35E-02 & $6.18 \mathrm{E}-02$ & $4.25 \mathrm{E}-02$ \\
\hline & 52 & 36.100 & $1.10 \mathrm{E}+11$ & $1.41 \mathrm{E}+11$ & $7.65 \mathrm{E}-03$ & $9.82 \mathrm{E}-03$ & $8.26 \mathrm{E}-02$ \\
\hline & 57 & 35.321 & $2.94 \mathrm{E}+09$ & $2.81 \mathrm{E}+09$ & $2.75 \mathrm{E}-03$ & $2.63 \mathrm{E}-03$ & $3.20 \mathrm{E}-04$ \\
\hline & 58 & 35.250 & $5.96 \mathrm{E}+10$ & $5.95 \mathrm{E}+10$ & $7.78 \mathrm{E}-02$ & $7.76 \mathrm{E}-02$ & $9.02 \mathrm{E}-03$ \\
\hline & 59 & 35.173 & $3.82 \mathrm{E}+12$ & $3.82 \mathrm{E}+12$ & $4.96 \mathrm{E}+00$ & $4.96 \mathrm{E}+00$ & $5.74 \mathrm{E}-01$ \\
\hline & 61 & 32.268 & $1.70 \mathrm{E}+09$ & $1.42 \mathrm{E}+09$ & $8.47 \mathrm{E}-05$ & 7.07E-05 & $6.65 \mathrm{E}-04$ \\
\hline & 68 & 31.058 & $1.62 \mathrm{E}+10$ & $1.68 \mathrm{E}+10$ & $1.64 \mathrm{E}-02$ & $1.70 \mathrm{E}-02$ & $1.68 \mathrm{E}-03$ \\
\hline & 83 & 29.946 & $1.67 \mathrm{E}+09$ & $1.79 \mathrm{E}+09$ & $1.57 \mathrm{E}-03$ & $1.68 \mathrm{E}-03$ & $1.55 \mathrm{E}-04$ \\
\hline & 96 & 29.693 & $2.41 \mathrm{E}+10$ & $2.54 \mathrm{E}+10$ & $2.23 \mathrm{E}-02$ & $2.35 \mathrm{E}-02$ & $2.18 \mathrm{E}-03$ \\
\hline & 110 & 24.158 & $9.82 \mathrm{E}+08$ & $9.18 \mathrm{E}+08$ & $4.30 \mathrm{E}-04$ & $4.02 \mathrm{E}-04$ & $3.42 \mathrm{E}-05$ \\
\hline & 111 & 24.124 & $6.42 \mathrm{E}+09$ & $6.20 \mathrm{E}+09$ & $3.92 \mathrm{E}-03$ & $3.79 \mathrm{E}-03$ & $3.11 \mathrm{E}-04$ \\
\hline & 112 & 24.101 & $1.35 \mathrm{E}+12$ & $1.33 \mathrm{E}+12$ & $8.21 \mathrm{E}-01$ & 8.09E-01 & $6.52 \mathrm{E}-02$ \\
\hline
\end{tabular}


Table 6. Cont.

\begin{tabular}{|c|c|c|c|c|c|c|c|}
\hline LL. & UL. & $\lambda$ & $A_{L}$ & $A_{V}$ & $f_{L}$ & $f_{V}$ & $S_{L}$ \\
\hline & 129 & 22.050 & $1.33 \mathrm{E}+10$ & $1.38 \mathrm{E}+10$ & $6.77 \mathrm{E}-03$ & $7.02 \mathrm{E}-03$ & 4.92E-04 \\
\hline & 131 & 22.018 & $3.53 \mathrm{E}+07$ & $2.98 \mathrm{E}+07$ & $1.80 \mathrm{E}-05$ & $1.52 \mathrm{E}-05$ & $1.30 \mathrm{E}-06$ \\
\hline & 146 & 21.470 & $1.06 \mathrm{E}+09$ & $1.16 \mathrm{E}+09$ & $5.12 \mathrm{E}-04$ & $5.60 \mathrm{E}-04$ & $3.62 \mathrm{E}-05$ \\
\hline & 157 & 21.417 & $6.90 \mathrm{E}+06$ & $6.23 \mathrm{E}+06$ & $3.32 \mathrm{E}-06$ & $3.00 \mathrm{E}-06$ & $2.34 \mathrm{E}-07$ \\
\hline & 159 & 21.406 & $1.64 \mathrm{E}+10$ & $1.72 \mathrm{E}+10$ & $7.88 \mathrm{E}-03$ & $8.28 \mathrm{E}-03$ & $5.56 \mathrm{E}-04$ \\
\hline & 161 & 21.402 & $1.26 \mathrm{E}+09$ & $1.34 \mathrm{E}+09$ & $6.04 \mathrm{E}-04$ & $6.44 \mathrm{E}-04$ & $4.26 \mathrm{E}-05$ \\
\hline & 168 & 11.232 & $8.91 \mathrm{E}+09$ & $1.08 \mathrm{E}+10$ & $1.87 \mathrm{E}-05$ & $2.26 \mathrm{E}-05$ & $6.10 \mathrm{E}-04$ \\
\hline & 179 & 10.907 & $1.82 \mathrm{E}+11$ & $1.72 \mathrm{E}+11$ & $2.28 \mathrm{E}-02$ & $2.15 \mathrm{E}-02$ & $8.18 \mathrm{E}-04$ \\
\hline \multirow[t]{11}{*}{21} & 27 & 584.609 & $7.85 \mathrm{E}+08$ & $6.25 \mathrm{E}+08$ & $1.21 \mathrm{E}-01$ & $9.61 \mathrm{E}-02$ & 2.32E-01 \\
\hline & 65 & 32.711 & $2.77 \mathrm{E}+11$ & $2.91 E+11$ & $1.33 \mathrm{E}-01$ & $1.40 \mathrm{E}-01$ & $1.44 \mathrm{E}-02$ \\
\hline & 76 & 32.409 & $5.28 \mathrm{E}+11$ & $5.41 \mathrm{E}+11$ & $2.50 \mathrm{E}-01$ & $2.55 \mathrm{E}-01$ & $2.66 \mathrm{E}-02$ \\
\hline & 79 & 31.303 & $2.96 \mathrm{E}+09$ & $2.40 \mathrm{E}+09$ & $1.31 \mathrm{E}-03$ & $1.06 \mathrm{E}-03$ & $1.35 \mathrm{E}-04$ \\
\hline & 93 & 31.186 & $2.96 \mathrm{E}+08$ & $2.13 \mathrm{E}+08$ & $1.30 \mathrm{E}-04$ & $9.30 \mathrm{E}-05$ & $1.33 \mathrm{E}-05$ \\
\hline & 97 & 30.515 & $1.20 \mathrm{E}+10$ & $1.19 \mathrm{E}+10$ & $5.01 \mathrm{E}-03$ & $4.99 \mathrm{E}-03$ & 5.03E-04 \\
\hline & 118 & 22.690 & $1.52 \mathrm{E}+11$ & $1.82 \mathrm{E}+11$ & $3.52 \mathrm{E}-02$ & 4.22E-02 & 2.63E-03 \\
\hline & 121 & 22.618 & $2.95 \mathrm{E}+11$ & $3.48 \mathrm{E}+11$ & $6.79 \mathrm{E}-02$ & 8.01E-02 & $5.06 \mathrm{E}-03$ \\
\hline & 138 & 21.998 & $9.01 \mathrm{E}+07$ & $6.85 \mathrm{E}+07$ & $1.96 \mathrm{E}-05$ & $1.49 \mathrm{E}-05$ & $1.42 \mathrm{E}-06$ \\
\hline & 154 & 21.832 & $4.77 \mathrm{E}+09$ & $4.85 \mathrm{E}+09$ & $1.02 \mathrm{E}-03$ & $1.04 \mathrm{E}-03$ & $7.35 \mathrm{E}-05$ \\
\hline & 172 & 11.152 & $1.19 \mathrm{E}+13$ & $1.19 \mathrm{E}+13$ & $6.63 \mathrm{E}-01$ & $6.63 \mathrm{E}-01$ & $2.43 \mathrm{E}-02$ \\
\hline \multirow[t]{20}{*}{22} & 30 & 392.125 & $5.87 \mathrm{E}+08$ & $7.30 \mathrm{E}+08$ & $5.05 \mathrm{E}-02$ & $6.28 \mathrm{E}-02$ & $6.52 \mathrm{E}-02$ \\
\hline & 48 & 38.176 & $2.32 \mathrm{E}+10$ & $2.25 \mathrm{E}+10$ & $5.08 \mathrm{E}-03$ & $4.92 \mathrm{E}-03$ & 6.38E-04 \\
\hline & 56 & 36.616 & $5.45 \mathrm{E}+10$ & $4.75 \mathrm{E}+10$ & $6.60 \mathrm{E}-03$ & $5.75 \mathrm{E}-03$ & 4.77E-02 \\
\hline & 63 & 32.806 & $5.11 \mathrm{E}+11$ & $5.23 \mathrm{E}+11$ & $2.53 \mathrm{E}-01$ & $2.59 \mathrm{E}-01$ & $2.73 \mathrm{E}-02$ \\
\hline & 65 & 32.502 & $2.26 \mathrm{E}+11$ & $2.23 \mathrm{E}+11$ & $1.07 \mathrm{E}-01$ & $1.06 \mathrm{E}-01$ & $1.15 \mathrm{E}-02$ \\
\hline & 72 & 31.939 & $3.12 \mathrm{E}+09$ & $3.04 \mathrm{E}+09$ & $2.51 \mathrm{E}-04$ & $2.44 \mathrm{E}-04$ & 2.32E-03 \\
\hline & 76 & 31.390 & $5.43 \mathrm{E}+10$ & $5.29 \mathrm{E}+10$ & $2.35 \mathrm{E}-02$ & $2.29 \mathrm{E}-02$ & $2.42 \mathrm{E}-03$ \\
\hline & 79 & 31.272 & $9.70 \mathrm{E}+09$ & $1.00 \mathrm{E}+10$ & $4.41 \mathrm{E}-03$ & $4.55 \mathrm{E}-03$ & $4.54 \mathrm{E}-04$ \\
\hline & 80 & 31.089 & $1.16 \mathrm{E}+10$ & $1.19 \mathrm{E}+10$ & 8.63E-04 & $8.82 \mathrm{E}-04$ & $8.62 \mathrm{E}-03$ \\
\hline & 82 & 30.844 & $5.64 \mathrm{E}+10$ & $4.42 \mathrm{E}+10$ & 8.04E-03 & $6.30 \mathrm{E}-03$ & 8.17E-04 \\
\hline & 89 & 30.695 & $4.84 \mathrm{E}+09$ & $4.81 \mathrm{E}+09$ & $3.46 \mathrm{E}-04$ & $3.43 \mathrm{E}-04$ & $3.40 \mathrm{E}-03$ \\
\hline & 93 & 30.597 & $8.29 \mathrm{E}+09$ & $8.28 \mathrm{E}+09$ & $3.49 \mathrm{E}-03$ & $3.49 \mathrm{E}-03$ & $3.51 \mathrm{E}-04$ \\
\hline & 94 & 30.589 & $8.23 \mathrm{E}+09$ & $8.27 \mathrm{E}+09$ & 5.81E-04 & $5.84 \mathrm{E}-04$ & $5.80 \mathrm{E}-03$ \\
\hline & 105 & 24.751 & $2.97 \mathrm{E}+10$ & $2.89 \mathrm{E}+10$ & $1.11 \mathrm{E}-03$ & $1.08 \mathrm{E}-03$ & $1.33 \mathrm{E}-02$ \\
\hline & 118 & 22.735 & $2.77 \mathrm{E}+11$ & $3.14 \mathrm{E}+11$ & 7.30E-02 & $8.28 \mathrm{E}-02$ & $5.47 \mathrm{E}-03$ \\
\hline & 120 & 22.673 & $4.29 \mathrm{E}+11$ & $4.73 \mathrm{E}+11$ & $1.23 \mathrm{E}-02$ & $1.36 \mathrm{E}-02$ & $1.82 \mathrm{E}-01$ \\
\hline & 121 & 22.663 & $1.34 \mathrm{E}+11$ & $1.50 \mathrm{E}+11$ & $3.48 \mathrm{E}-02$ & $3.90 \mathrm{E}-02$ & $2.59 \mathrm{E}-03$ \\
\hline & 127 & 22.532 & $8.69 E+08$ & $7.03 E+08$ & $2.45 \mathrm{E}-05$ & $1.98 \mathrm{E}-05$ & $2.68 \mathrm{E}-04$ \\
\hline & 136 & 22.072 & $1.11 \mathrm{E}+09$ & $1.43 \mathrm{E}+09$ & $2.94 \mathrm{E}-05$ & 3.79E-05 & $5.22 \mathrm{E}-04$ \\
\hline & 137 & 22.071 & $2.41 \mathrm{E}+10$ & $2.07 \mathrm{E}+10$ & $4.54 \mathrm{E}-03$ & $3.90 \mathrm{E}-03$ & 3.30E-04 \\
\hline
\end{tabular}


Table 6. Cont.

\begin{tabular}{|c|c|c|c|c|c|c|c|}
\hline LL. & UL. & $\lambda$ & $A_{L}$ & $A_{V}$ & $f_{L}$ & $f_{V}$ & $S_{L}$ \\
\hline & 138 & 22.041 & $5.88 \mathrm{E}+09$ & $5.12 \mathrm{E}+09$ & $1.12 \mathrm{E}-03$ & $9.75 \mathrm{E}-04$ & $8.12 \mathrm{E}-05$ \\
\hline & 140 & 22.010 & $1.06 \mathrm{E}+10$ & $1.12 \mathrm{E}+10$ & 2.79E-04 & $2.95 \mathrm{E}-04$ & $4.07 \mathrm{E}-03$ \\
\hline & 147 & 21.905 & $2.22 \mathrm{E}+09$ & $2.09 \mathrm{E}+09$ & $5.75 \mathrm{E}-05$ & $5.41 \mathrm{E}-05$ & $7.50 \mathrm{E}-04$ \\
\hline & 151 & 21.879 & $2.65 \mathrm{E}+09$ & $3.06 \mathrm{E}+09$ & $6.85 \mathrm{E}-05$ & $7.90 \mathrm{E}-05$ & $1.10 \mathrm{E}-03$ \\
\hline & 154 & 21.874 & $3.39 \mathrm{E}+09$ & $3.38 \mathrm{E}+09$ & 7.26E-04 & $7.24 \mathrm{E}-04$ & $5.23 \mathrm{E}-05$ \\
\hline & 171 & 11.195 & $2.30 \mathrm{E}+12$ & $1.87 \mathrm{E}+12$ & 7.95E-03 & $6.47 \mathrm{E}-03$ & $1.76 \mathrm{E}-01$ \\
\hline & 172 & 11.163 & $7.38 \mathrm{E}+12$ & $7.36 \mathrm{E}+12$ & 4.12E-01 & 4.11E-01 & $1.51 \mathrm{E}-02$ \\
\hline & 176 & 11.119 & $2.49 \mathrm{E}+10$ & $2.40 \mathrm{E}+10$ & $1.33 \mathrm{E}-03$ & $1.28 \mathrm{E}-03$ & $4.88 \mathrm{E}-05$ \\
\hline \multirow[t]{21}{*}{23} & 38 & 373.023 & $3.05 \mathrm{E}+06$ & $2.69 \mathrm{E}+06$ & $3.18 \mathrm{E}-04$ & $2.81 \mathrm{E}-04$ & $3.90 \mathrm{E}-04$ \\
\hline & 57 & 37.850 & $3.69 \mathrm{E}+10$ & $3.76 \mathrm{E}+10$ & $3.96 \mathrm{E}-02$ & 4.04E-02 & 4.93E-03 \\
\hline & 61 & 34.366 & $1.29 \mathrm{E}+11$ & $1.32 \mathrm{E}+11$ & 7.02E-02 & $7.18 \mathrm{E}-02$ & 7.94E-03 \\
\hline & 62 & 34.350 & $2.12 \mathrm{E}+11$ & $2.13 \mathrm{E}+11$ & $3.76 \mathrm{E}-02$ & $3.77 \mathrm{E}-02$ & $4.25 \mathrm{E}-03$ \\
\hline & 66 & 33.279 & $1.72 \mathrm{E}+12$ & $1.81 \mathrm{E}+12$ & $1.43 \mathrm{E}+00$ & $1.50 \mathrm{E}+00$ & $1.56 \mathrm{E}-01$ \\
\hline & 67 & 33.097 & $7.09 \mathrm{E}+07$ & $7.98 \mathrm{E}+07$ & 5.82E-05 & $6.55 \mathrm{E}-05$ & $6.34 \mathrm{E}-06$ \\
\hline & 69 & 32.995 & $6.08 \mathrm{E}+11$ & $5.71 \mathrm{E}+11$ & $2.80 \mathrm{E}-01$ & $2.63 \mathrm{E}-01$ & $3.04 \mathrm{E}-02$ \\
\hline & 71 & 32.928 & $2.88 \mathrm{E}+09$ & $3.15 \mathrm{E}+09$ & 2.34E-03 & $2.56 \mathrm{E}-03$ & $2.54 \mathrm{E}-04$ \\
\hline & 75 & 32.866 & $1.06 \mathrm{E}+10$ & $1.01 \mathrm{E}+10$ & 4.90E-03 & $4.67 \mathrm{E}-03$ & $5.30 \mathrm{E}-04$ \\
\hline & 84 & 31.730 & $4.19 \mathrm{E}+07$ & $3.81 \mathrm{E}+08$ & $1.73 \mathrm{E}-04$ & $1.57 \mathrm{E}-03$ & $1.80 \mathrm{E}-05$ \\
\hline & 95 & 31.471 & $2.29 \mathrm{E}+10$ & $1.94 \mathrm{E}+10$ & 8.64E-03 & 7.32E-03 & 8.95E-04 \\
\hline & 110 & 25.314 & $1.16 \mathrm{E}+10$ & $1.27 \mathrm{E}+10$ & $5.58 \mathrm{E}-03$ & $6.10 \mathrm{E}-03$ & $4.65 \mathrm{E}-04$ \\
\hline & 116 & 23.363 & $6.49 \mathrm{E}+10$ & $7.70 \mathrm{E}+10$ & $1.89 \mathrm{E}-02$ & $2.24 \mathrm{E}-02$ & $1.45 \mathrm{E}-03$ \\
\hline & 117 & 23.332 & $1.11 \mathrm{E}+11$ & $1.24 \mathrm{E}+11$ & $9.03 \mathrm{E}-03$ & $1.01 \mathrm{E}-02$ & $6.94 \mathrm{E}-04$ \\
\hline & 122 & 23.097 & $8.76 \mathrm{E}+11$ & $9.05 \mathrm{E}+11$ & $3.50 \mathrm{E}-01$ & $3.62 \mathrm{E}-01$ & $2.66 \mathrm{E}-02$ \\
\hline & 124 & 23.048 & $4.76 \mathrm{E}+08$ & $4.84 \mathrm{E}+08$ & $1.90 \mathrm{E}-04$ & $1.93 \mathrm{E}-04$ & $1.44 \mathrm{E}-05$ \\
\hline & 125 & 23.035 & $3.33 \mathrm{E}+11$ & $3.19 \mathrm{E}+11$ & 7.61E-02 & 7.29E-02 & $5.77 \mathrm{E}-03$ \\
\hline & 134 & 22.675 & $1.70 \mathrm{E}+09$ & $1.54 \mathrm{E}+09$ & $3.56 \mathrm{E}-04$ & $3.22 \mathrm{E}-04$ & $2.66 \mathrm{E}-05$ \\
\hline & 141 & 22.426 & $3.69 \mathrm{E}+09$ & $3.87 \mathrm{E}+09$ & $1.39 \mathrm{E}-03$ & $1.46 \mathrm{E}-03$ & $1.03 \mathrm{E}-04$ \\
\hline & 152 & 22.337 & $8.51 \mathrm{E}+09$ & $7.80 \mathrm{E}+09$ & $1.75 \mathrm{E}-03$ & $1.60 \mathrm{E}-03$ & $1.29 \mathrm{E}-04$ \\
\hline & 180 & 11.171 & $1.79 E+13$ & $1.46 \mathrm{E}+13$ & $1.67 \mathrm{E}+00$ & $1.37 \mathrm{E}+00$ & $6.16 \mathrm{E}-02$ \\
\hline \multirow[t]{10}{*}{24} & 63 & 34.018 & $4.44 \mathrm{E}+08$ & $4.55 \mathrm{E}+08$ & 2.31E-04 & 2.37E-04 & $2.59 \mathrm{E}-05$ \\
\hline & 64 & 33.706 & $3.47 \mathrm{E}+09$ & $2.99 \mathrm{E}+09$ & $2.96 \mathrm{E}-03$ & $2.54 \mathrm{E}-03$ & $3.28 \mathrm{E}-04$ \\
\hline & 72 & 33.087 & $3.92 \mathrm{E}+09$ & $3.99 \mathrm{E}+09$ & $3.22 \mathrm{E}-03$ & $3.28 \mathrm{E}-03$ & $3.51 \mathrm{E}-04$ \\
\hline & 73 & 33.086 & $1.51 \mathrm{E}+09$ & $1.52 \mathrm{E}+09$ & $1.74 \mathrm{E}-03$ & $1.75 \mathrm{E}-03$ & $1.89 \mathrm{E}-04$ \\
\hline & 74 & 33.038 & $4.33 \mathrm{E}+10$ & $4.35 \mathrm{E}+10$ & 4.96E-02 & $4.98 \mathrm{E}-02$ & $5.39 \mathrm{E}-03$ \\
\hline & 76 & 32.498 & $1.91 \mathrm{E}+11$ & $1.90 \mathrm{E}+11$ & $9.09 \mathrm{E}-02$ & $9.01 \mathrm{E}-02$ & $9.73 \mathrm{E}-03$ \\
\hline & 77 & 32.451 & $6.46 \mathrm{E}+11$ & $6.42 \mathrm{E}+11$ & $5.10 \mathrm{E}-01$ & $5.07 \mathrm{E}-01$ & $5.45 \mathrm{E}-02$ \\
\hline & 78 & 32.373 & $8.35 E+11$ & $8.35 E+11$ & 9.19E-01 & $9.18 \mathrm{E}-01$ & $9.79 \mathrm{E}-02$ \\
\hline & 79 & 32.372 & $6.18 \mathrm{E}+11$ & $6.06 \mathrm{E}+11$ & $2.91 \mathrm{E}-01$ & $2.85 \mathrm{E}-01$ & $3.11 \mathrm{E}-02$ \\
\hline & 80 & 32.175 & $1.85 \mathrm{E}+11$ & $1.86 \mathrm{E}+11$ & $1.44 \mathrm{E}-01$ & $1.44 \mathrm{E}-01$ & $1.52 \mathrm{E}-02$ \\
\hline
\end{tabular}


Table 6. Cont.

\begin{tabular}{|c|c|c|c|c|c|c|c|}
\hline LL. & UL. & $\lambda$ & $A_{L}$ & $A_{V}$ & $f_{L}$ & $f_{V}$ & $S_{L}$ \\
\hline & 89 & 31.753 & $3.40 \mathrm{E}+09$ & $3.42 \mathrm{E}+09$ & $2.57 \mathrm{E}-03$ & $2.58 \mathrm{E}-03$ & $2.68 \mathrm{E}-04$ \\
\hline & 91 & 31.739 & $1.42 \mathrm{E}+08$ & $1.41 \mathrm{E}+08$ & $1.50 \mathrm{E}-04$ & 1.49E-04 & $1.57 \mathrm{E}-05$ \\
\hline & 93 & 31.649 & $1.08 \mathrm{E}+09$ & $1.08 \mathrm{E}+09$ & $4.85 \mathrm{E}-04$ & $4.88 \mathrm{E}-04$ & $5.06 \mathrm{E}-05$ \\
\hline & 94 & 31.640 & $4.41 \mathrm{E}+09$ & $4.45 \mathrm{E}+09$ & $3.31 \mathrm{E}-03$ & 3.34E-03 & $3.45 \mathrm{E}-04$ \\
\hline & 105 & 25.435 & $6.88 \mathrm{E}+04$ & $9.46 \mathrm{E}+05$ & 3.34E-08 & 4.59E-07 & 2.79E-09 \\
\hline & 118 & 23.311 & $1.36 \mathrm{E}+08$ & $1.03 \mathrm{E}+08$ & 3.32E-05 & $2.52 \mathrm{E}-05$ & $2.55 \mathrm{E}-06$ \\
\hline & 120 & 23.245 & $1.09 \mathrm{E}+08$ & $6.12 \mathrm{E}+07$ & 8.78E-06 & 4.96E-06 & $3.37 \mathrm{E}-06$ \\
\hline & 121 & 23.235 & $3.81 \mathrm{E}+08$ & $3.24 \mathrm{E}+08$ & $9.25 \mathrm{E}-05$ & 7.87E-05 & 7.07E-06 \\
\hline & 127 & 23.097 & $1.92 \mathrm{E}+09$ & $1.92 \mathrm{E}+09$ & $1.54 \mathrm{E}-04$ & $1.54 \mathrm{E}-04$ & $5.83 \mathrm{E}-05$ \\
\hline & 136 & 22.614 & $1.24 \mathrm{E}+11$ & $1.12 \mathrm{E}+11$ & 4.73E-02 & 4.26E-02 & $3.51 \mathrm{E}-03$ \\
\hline & 137 & 22.548 & $1.24 \mathrm{E}+11$ & $1.12 \mathrm{E}+11$ & $9.46 \mathrm{E}-03$ & $8.52 \mathrm{E}-03$ & $3.51 \mathrm{E}-03$ \\
\hline & 138 & 22.438 & $1.41 \mathrm{E}+09$ & $1.39 \mathrm{E}+09$ & $1.06 \mathrm{E}-02$ & $1.05 \mathrm{E}-02$ & $3.93 \mathrm{E}-05$ \\
\hline & 154 & 22.406 & $4.78 \mathrm{E}+08$ & $4.76 \mathrm{E}+08$ & $1.08 \mathrm{E}-04$ & 1.07E-04 & 7.97E-06 \\
\hline & 172 & 11.300 & $5.67 \mathrm{E}+11$ & $5.62 \mathrm{E}+11$ & $3.25 \mathrm{E}-02$ & 3.23E-02 & $1.21 \mathrm{E}-03$ \\
\hline \multirow[t]{20}{*}{25} & 48 & 40.587 & $2.15 \mathrm{E}+10$ & $1.68 \mathrm{E}+10$ & $5.30 \mathrm{E}-03$ & 4.14E-03 & 7.08E-04 \\
\hline & 63 & 34.570 & $2.99 \mathrm{E}+10$ & $3.16 \mathrm{E}+10$ & $1.70 \mathrm{E}-02$ & $1.80 \mathrm{E}-02$ & $1.93 \mathrm{E}-03$ \\
\hline & 64 & 34.249 & $5.61 \mathrm{E}+09$ & $4.93 \mathrm{E}+09$ & 5.56E-04 & 4.89E-04 & 4.34E-03 \\
\hline & 65 & 34.234 & $2.97 \mathrm{E}+10$ & $3.22 \mathrm{E}+10$ & 1.69E-02 & $1.83 \mathrm{E}-02$ & $1.91 \mathrm{E}-03$ \\
\hline & 76 & 33.002 & $5.76 \mathrm{E}+11$ & $5.94 \mathrm{E}+11$ & $2.91 \mathrm{E}-01$ & $3.00 \mathrm{E}-01$ & $3.16 \mathrm{E}-02$ \\
\hline & 77 & 32.954 & $2.05 \mathrm{E}+11$ & $2.07 \mathrm{E}+11$ & $1.81 \mathrm{E}-02$ & $1.82 \mathrm{E}-02$ & $1.68 \mathrm{E}-01$ \\
\hline & 79 & 32.872 & $1.54 \mathrm{E}+11$ & $1.62 \mathrm{E}+11$ & 7.86E-02 & 8.27E-02 & $8.50 \mathrm{E}-03$ \\
\hline & 80 & 32.669 & $6.22 \mathrm{E}+11$ & $6.16 \mathrm{E}+11$ & 5.35E-02 & $5.30 \mathrm{E}-02$ & 4.93E-01 \\
\hline & 82 & 32.399 & $6.64 \mathrm{E}+11$ & $7.56 \mathrm{E}+11$ & $1.04 \mathrm{E}-01$ & 1.19E-01 & $1.11 \mathrm{E}-02$ \\
\hline & 89 & 32.234 & $6.41 \mathrm{E}+09$ & $6.44 \mathrm{E}+09$ & 5.29E-04 & 5.32E-04 & $5.01 \mathrm{E}-03$ \\
\hline & 93 & 32.127 & $3.60 \mathrm{E}+09$ & $3.60 \mathrm{E}+09$ & 1.67E-03 & $1.67 \mathrm{E}-03$ & $1.77 \mathrm{E}-04$ \\
\hline & 105 & 25.742 & $6.19 \mathrm{E}+10$ & $5.58 \mathrm{E}+10$ & 2.61E-03 & $2.35 \mathrm{E}-03$ & $2.77 \mathrm{E}-02$ \\
\hline & 120 & 23.502 & $1.09 \mathrm{E}+10$ & $1.20 \mathrm{E}+10$ & $3.49 \mathrm{E}-04$ & 3.84E-04 & $4.96 \mathrm{E}-03$ \\
\hline & 136 & 22.857 & $1.17 \mathrm{E}+11$ & $1.28 \mathrm{E}+11$ & $3.46 \mathrm{E}-03$ & $3.76 \mathrm{E}-03$ & $5.00 \mathrm{E}-02$ \\
\hline & 137 & 22.856 & $3.00 \mathrm{E}+11$ & $3.64 \mathrm{E}+11$ & 8.56E-02 & $1.04 \mathrm{E}-01$ & $6.44 \mathrm{E}-03$ \\
\hline & 138 & 22.823 & $9.75 E+10$ & $1.20 \mathrm{E}+11$ & $2.81 \mathrm{E}-02$ & $3.46 \mathrm{E}-02$ & $2.11 \mathrm{E}-03$ \\
\hline & 140 & 22.790 & $3.36 \mathrm{E}+11$ & $3.68 \mathrm{E}+11$ & $9.82 \mathrm{E}-03$ & $1.07 \mathrm{E}-02$ & $1.43 \mathrm{E}-01$ \\
\hline & 147 & 22.678 & $3.39 \mathrm{E}+09$ & $3.98 \mathrm{E}+09$ & $9.77 \mathrm{E}-05$ & $1.15 \mathrm{E}-04$ & $1.53 \mathrm{E}-03$ \\
\hline & 154 & 22.645 & $1.58 \mathrm{E}+09$ & $1.65 \mathrm{E}+09$ & $3.80 \mathrm{E}-04$ & 3.97E-04 & $2.83 \mathrm{E}-05$ \\
\hline & 172 & 11.360 & $1.39 \mathrm{E}+12$ & $1.40 \mathrm{E}+12$ & 8.10E-02 & $8.16 \mathrm{E}-02$ & $3.03 \mathrm{E}-03$ \\
\hline \multirow[t]{5}{*}{26} & 46 & 312.483 & $2.49 \mathrm{E}+07$ & $2.36 \mathrm{E}+07$ & $1.12 \mathrm{E}-03$ & $1.07 \mathrm{E}-03$ & $1.04 \mathrm{E}-03$ \\
\hline & 52 & 39.719 & $5.32 \mathrm{E}+07$ & $6.71 \mathrm{E}+08$ & 4.93E-06 & $6.22 \mathrm{E}-05$ & $4.76 \mathrm{E}-04$ \\
\hline & 57 & 38.779 & $5.20 \mathrm{E}+09$ & $5.31 \mathrm{E}+09$ & $5.86 \mathrm{E}-03$ & $5.99 \mathrm{E}-03$ & 7.49E-04 \\
\hline & 61 & 35.130 & $3.47 \mathrm{E}+11$ & $3.67 \mathrm{E}+11$ & $2.23 \mathrm{E}-02$ & $2.36 \mathrm{E}-02$ & $2.04 \mathrm{E}-01$ \\
\hline & 66 & 33.995 & $2.70 \mathrm{E}+11$ & $2.73 \mathrm{E}+11$ & 2.34E-01 & $2.36 \mathrm{E}-01$ & $2.62 \mathrm{E}-02$ \\
\hline
\end{tabular}


Table 6. Cont.

\begin{tabular}{|c|c|c|c|c|c|c|c|}
\hline LL. & UL. & $\lambda$ & $A_{L}$ & $A_{V}$ & $f_{L}$ & $f_{V}$ & $S_{L}$ \\
\hline & 67 & 33.805 & $9.61 \mathrm{E}+10$ & $8.39 \mathrm{E}+10$ & $8.23 \mathrm{E}-02$ & $7.18 \mathrm{E}-02$ & $9.16 \mathrm{E}-03$ \\
\hline & 69 & 33.699 & $4.00 \mathrm{E}+10$ & $4.39 \mathrm{E}+10$ & $2.27 \mathrm{E}-03$ & $2.49 \mathrm{E}-03$ & $2.24 \mathrm{E}-02$ \\
\hline & 71 & 33.629 & $9.56 \mathrm{E}+10$ & $9.34 \mathrm{E}+10$ & $8.10 \mathrm{E}-02$ & 7.91E-02 & 8.97E-03 \\
\hline & 75 & 33.564 & $3.03 \mathrm{E}+10$ & $2.83 \mathrm{E}+10$ & $1.70 \mathrm{E}-03$ & $1.59 \mathrm{E}-03$ & $1.44 \mathrm{E}-02$ \\
\hline & 94 & 32.118 & $2.15 \mathrm{E}+08$ & $1.96 \mathrm{E}+08$ & $1.76 \mathrm{E}-05$ & $1.60 \mathrm{E}-05$ & $1.51 \mathrm{E}-04$ \\
\hline & 110 & 25.726 & $1.57 \mathrm{E}+09$ & $1.73 \mathrm{E}+09$ & $7.80 \mathrm{E}-04$ & $8.60 \mathrm{E}-04$ & $6.61 \mathrm{E}-05$ \\
\hline & 116 & 23.714 & $1.63 \mathrm{E}+11$ & $2.01 \mathrm{E}+11$ & $3.22 \mathrm{E}-03$ & $3.97 \mathrm{E}-03$ & $5.09 \mathrm{E}-02$ \\
\hline & 122 & 23.440 & $1.31 \mathrm{E}+11$ & $1.30 \mathrm{E}+11$ & 5.38E-02 & 5.37E-02 & $4.16 \mathrm{E}-03$ \\
\hline & 124 & 23.389 & $8.61 \mathrm{E}+10$ & $8.64 \mathrm{E}+10$ & $3.53 \mathrm{E}-02$ & $3.54 \mathrm{E}-02$ & $2.72 \mathrm{E}-03$ \\
\hline & 125 & 23.375 & $2.06 \mathrm{E}+10$ & $2.25 \mathrm{E}+10$ & $3.90 \mathrm{E}-04$ & $4.26 \mathrm{E}-04$ & $5.54 \mathrm{E}-03$ \\
\hline & 134 & 23.022 & $9.20 \mathrm{E}+09$ & $1.02 \mathrm{E}+10$ & $3.66 \mathrm{E}-03$ & $4.05 \mathrm{E}-03$ & $2.77 \mathrm{E}-04$ \\
\hline & 135 & 23.005 & $1.67 \mathrm{E}+10$ & $1.56 \mathrm{E}+10$ & $3.01 \mathrm{E}-04$ & $2.81 \mathrm{E}-04$ & $3.71 \mathrm{E}-03$ \\
\hline & 141 & 22.749 & $5.07 \mathrm{E}+10$ & $5.91 \mathrm{E}+10$ & $1.97 \mathrm{E}-02$ & $2.29 \mathrm{E}-02$ & $1.47 \mathrm{E}-03$ \\
\hline \multirow[t]{15}{*}{27} & 36 & 779.462 & $2.61 \mathrm{E}+07$ & $3.11 \mathrm{E}+07$ & $8.49 \mathrm{E}-03$ & $1.01 \mathrm{E}-02$ & $2.18 \mathrm{E}-02$ \\
\hline & 43 & 390.850 & $2.98 \mathrm{E}+08$ & $2.13 E+08$ & $6.82 \mathrm{E}-03$ & 4.87E-03 & 8.77E-03 \\
\hline & 49 & 40.079 & $1.43 \mathrm{E}+09$ & $1.66 \mathrm{E}+09$ & $1.20 \mathrm{E}-03$ & $1.39 \mathrm{E}-03$ & $1.58 \mathrm{E}-04$ \\
\hline & 61 & 35.191 & $5.01 \mathrm{E}+10$ & $5.35 \mathrm{E}+10$ & $2.98 \mathrm{E}-02$ & $3.18 \mathrm{E}-02$ & $3.45 \mathrm{E}-03$ \\
\hline & 62 & 35.174 & $4.48 \mathrm{E}+11$ & $4.74 \mathrm{E}+11$ & $8.30 \mathrm{E}-02$ & 8.79E-02 & $9.62 \mathrm{E}-03$ \\
\hline & 69 & 33.755 & $1.93 \mathrm{E}+11$ & $1.84 \mathrm{E}+11$ & $9.44 \mathrm{E}-02$ & $9.00 \mathrm{E}-02$ & $1.05 \mathrm{E}-02$ \\
\hline & 75 & 33.620 & $3.06 \mathrm{E}+10$ & $2.85 \mathrm{E}+10$ & $1.45 \mathrm{E}-02$ & $1.35 \mathrm{E}-02$ & $1.60 \mathrm{E}-03$ \\
\hline & 84 & 32.432 & $6.72 \mathrm{E}+10$ & $5.61 \mathrm{E}+10$ & $2.65 \mathrm{E}-02$ & $2.21 \mathrm{E}-02$ & $2.83 \mathrm{E}-03$ \\
\hline & 95 & 32.162 & $9.52 \mathrm{E}+10$ & $8.39 \mathrm{E}+10$ & $3.90 \mathrm{E}-02$ & $3.44 \mathrm{E}-02$ & $4.13 \mathrm{E}-03$ \\
\hline & 116 & 23.742 & $2.68 \mathrm{E}+10$ & $3.39 \mathrm{E}+10$ & $8.58 \mathrm{E}-03$ & $1.09 \mathrm{E}-02$ & $6.71 \mathrm{E}-04$ \\
\hline & 117 & 23.710 & $2.32 \mathrm{E}+11$ & $2.76 \mathrm{E}+11$ & $1.95 \mathrm{E}-02$ & $2.33 \mathrm{E}-02$ & $1.52 \mathrm{E}-03$ \\
\hline & 125 & 23.403 & $1.14 \mathrm{E}+11$ & $1.15 \mathrm{E}+11$ & $2.83 \mathrm{E}-02$ & $2.85 \mathrm{E}-02$ & $2.18 \mathrm{E}-03$ \\
\hline & 134 & 23.032 & $1.13 \mathrm{E}+10$ & $1.02 \mathrm{E}+10$ & $2.43 \mathrm{E}-03$ & $2.19 \mathrm{E}-03$ & $1.84 \mathrm{E}-04$ \\
\hline & 143 & 22.743 & $3.47 \mathrm{E}+10$ & $2.99 \mathrm{E}+10$ & $6.97 \mathrm{E}-03$ & $6.01 \mathrm{E}-03$ & $5.22 \mathrm{E}-04$ \\
\hline & 152 & 22.682 & $4.72 \mathrm{E}+10$ & $4.16 \mathrm{E}+10$ & $9.62 \mathrm{E}-03$ & $8.48 \mathrm{E}-03$ & $7.18 \mathrm{E}-04$ \\
\hline \multirow[t]{8}{*}{28} & 75 & 34.102 & $1.93 \mathrm{E}+10$ & $1.90 \mathrm{E}+10$ & $1.01 \mathrm{E}-02$ & $9.91 \mathrm{E}-03$ & $1.13 \mathrm{E}-03$ \\
\hline & 84 & 32.879 & $2.24 \mathrm{E}+08$ & $1.93 \mathrm{E}+08$ & $1.09 \mathrm{E}-04$ & $9.38 \mathrm{E}-05$ & $1.18 \mathrm{E}-05$ \\
\hline & 95 & 32.602 & $5.87 \mathrm{E}+10$ & $4.50 \mathrm{E}+10$ & $2.81 \mathrm{E}-02$ & $2.15 \mathrm{E}-02$ & $3.01 \mathrm{E}-03$ \\
\hline & 125 & 23.635 & $7.91 \mathrm{E}+11$ & $5.46 \mathrm{E}+11$ & $1.99 \mathrm{E}-01$ & $1.37 \mathrm{E}-01$ & $1.55 \mathrm{E}-02$ \\
\hline & 134 & 23.257 & $6.16 \mathrm{E}+09$ & $4.50 \mathrm{E}+09$ & $1.50 \mathrm{E}-03$ & $1.09 \mathrm{E}-03$ & $1.15 \mathrm{E}-04$ \\
\hline & 143 & 22.962 & $2.59 \mathrm{E}+08$ & $2.77 \mathrm{E}+08$ & $6.14 \mathrm{E}-05$ & $6.56 \mathrm{E}-05$ & $4.64 \mathrm{E}-06$ \\
\hline & 152 & 22.900 & $3.26 \mathrm{E}+10$ & $3.03 \mathrm{E}+10$ & 7.69E-03 & $7.15 \mathrm{E}-03$ & $5.80 \mathrm{E}-04$ \\
\hline & 170 & 11.464 & $6.65 \mathrm{E}+10$ & $6.96 \mathrm{E}+10$ & $3.93 \mathrm{E}-03$ & $4.11 \mathrm{E}-03$ & $1.48 \mathrm{E}-04$ \\
\hline \multirow[t]{3}{*}{29} & 64 & 34.984 & $8.93 \mathrm{E}+09$ & $8.99 \mathrm{E}+09$ & $8.19 \mathrm{E}-03$ & $8.25 \mathrm{E}-03$ & $9.43 \mathrm{E}-04$ \\
\hline & 65 & 34.968 & $2.13 \mathrm{E}+09$ & $2.35 \mathrm{E}+09$ & 1.17E-03 & $1.29 \mathrm{E}-03$ & $1.35 \mathrm{E}-04$ \\
\hline & 67 & 34.960 & $1.23 \mathrm{E}+11$ & $1.03 E+11$ & $1.13 \mathrm{E}-01$ & $9.42 \mathrm{E}-02$ & $1.30 \mathrm{E}-02$ \\
\hline
\end{tabular}


Table 6. Cont.

\begin{tabular}{|c|c|c|c|c|c|c|c|}
\hline LL. & UL. & $\lambda$ & $A_{L}$ & $A_{V}$ & $f_{L}$ & $f_{V}$ & $S_{L}$ \\
\hline & 71 & 34.772 & $1.56 \mathrm{E}+09$ & $1.65 \mathrm{E}+09$ & $1.41 \mathrm{E}-03$ & $1.50 \mathrm{E}-03$ & $1.62 \mathrm{E}-04$ \\
\hline & 72 & 34.317 & $3.87 \mathrm{E}+11$ & $3.89 \mathrm{E}+11$ & $3.41 \mathrm{E}-01$ & $3.43 \mathrm{E}-01$ & $3.86 \mathrm{E}-02$ \\
\hline & 73 & 34.317 & $3.72 \mathrm{E}+12$ & $3.76 \mathrm{E}+12$ & $4.60 \mathrm{E}+00$ & $4.64 \mathrm{E}+00$ & $5.20 \mathrm{E}-01$ \\
\hline & 74 & 34.265 & $1.24 \mathrm{E}+08$ & $1.30 \mathrm{E}+08$ & $1.53 \mathrm{E}-04$ & $1.60 \mathrm{E}-04$ & $1.73 \mathrm{E}-05$ \\
\hline & 77 & 33.634 & $1.46 \mathrm{E}+07$ & $1.59 \mathrm{E}+07$ & $1.23 \mathrm{E}-05$ & $1.35 \mathrm{E}-05$ & $1.37 \mathrm{E}-06$ \\
\hline & 78 & 33.550 & $1.96 \mathrm{E}+08$ & $1.57 \mathrm{E}+08$ & $2.31 \mathrm{E}-04$ & $1.85 \mathrm{E}-04$ & $2.55 \mathrm{E}-05$ \\
\hline & 80 & 33.338 & $1.62 \mathrm{E}+09$ & $1.47 \mathrm{E}+09$ & $1.35 \mathrm{E}-03$ & $1.23 \mathrm{E}-03$ & $1.48 \mathrm{E}-04$ \\
\hline & 89 & 32.885 & $1.88 \mathrm{E}+09$ & $1.91 \mathrm{E}+09$ & $1.52 \mathrm{E}-03$ & $1.55 \mathrm{E}-03$ & $1.65 \mathrm{E}-04$ \\
\hline & 91 & 32.870 & $1.27 \mathrm{E}+10$ & $1.34 \mathrm{E}+10$ & $1.44 \mathrm{E}-02$ & $1.52 \mathrm{E}-02$ & $1.56 \mathrm{E}-03$ \\
\hline & 93 & 32.773 & $1.52 \mathrm{E}+09$ & $1.31 \mathrm{E}+09$ & 7.37E-04 & $6.35 \mathrm{E}-04$ & 7.95E-05 \\
\hline & 94 & 32.764 & $4.80 \mathrm{E}+09$ & $4.93 \mathrm{E}+09$ & $3.86 \mathrm{E}-03$ & $3.96 \mathrm{E}-03$ & $4.17 \mathrm{E}-04$ \\
\hline & 110 & 26.390 & $5.21 \mathrm{E}+08$ & $6.10 \mathrm{E}+08$ & 2.72E-04 & $3.18 \mathrm{E}-04$ & $2.36 \mathrm{E}-05$ \\
\hline & 120 & 23.690 & $1.40 \mathrm{E}+11$ & $1.37 \mathrm{E}+11$ & $5.88 \mathrm{E}-02$ & $5.75 \mathrm{E}-02$ & $4.59 \mathrm{E}-03$ \\
\hline & 136 & 23.113 & $5.41 \mathrm{E}+08$ & $5.14 \mathrm{E}+08$ & $2.17 \mathrm{E}-04$ & $2.06 \mathrm{E}-04$ & $1.65 \mathrm{E}-05$ \\
\hline & 172 & 11.440 & $8.56 \mathrm{E}+11$ & $7.69 \mathrm{E}+11$ & $5.04 \mathrm{E}-02$ & $4.53 \mathrm{E}-02$ & $1.90 \mathrm{E}-03$ \\
\hline \multirow[t]{15}{*}{30} & 43 & 613.306 & $4.01 \mathrm{E}+08$ & $4.15 \mathrm{E}+07$ & $2.26 \mathrm{E}-02$ & $2.34 \mathrm{E}-03$ & $4.57 \mathrm{E}-02$ \\
\hline & 61 & 36.379 & $1.73 E+10$ & $2.08 \mathrm{E}+10$ & $1.24 \mathrm{E}-02$ & $1.49 \mathrm{E}-02$ & $1.48 \mathrm{E}-03$ \\
\hline & 62 & 36.361 & $3.97 \mathrm{E}+10$ & $5.15 \mathrm{E}+10$ & $7.86 \mathrm{E}-03$ & $1.02 \mathrm{E}-02$ & $9.41 \mathrm{E}-04$ \\
\hline & 63 & 35.320 & $1.08 \mathrm{E}+11$ & $1.11 \mathrm{E}+11$ & $6.04 \mathrm{E}-02$ & $6.22 \mathrm{E}-02$ & $7.02 \mathrm{E}-03$ \\
\hline & 69 & 34.847 & $1.07 \mathrm{E}+11$ & $1.14 \mathrm{E}+11$ & $6.22 \mathrm{E}-02$ & $6.63 \mathrm{E}-02$ & $7.14 \mathrm{E}-03$ \\
\hline & 75 & 34.703 & $1.55 \mathrm{E}+11$ & $1.61 \mathrm{E}+11$ & 8.72E-02 & $9.06 \mathrm{E}-02$ & $9.96 \mathrm{E}-03$ \\
\hline & 84 & 33.438 & $1.04 \mathrm{E}+11$ & $1.21 \mathrm{E}+11$ & $6.06 \mathrm{E}-02$ & 7.05E-02 & $6.67 \mathrm{E}-03$ \\
\hline & 88 & 33.355 & $8.24 \mathrm{E}+10$ & $9.54 \mathrm{E}+10$ & $1.37 \mathrm{E}-02$ & $1.59 \mathrm{E}-02$ & $1.51 \mathrm{E}-03$ \\
\hline & 95 & 33.152 & $6.75 \mathrm{E}+11$ & $6.27 \mathrm{E}+11$ & $3.10 \mathrm{E}-01$ & $2.88 \mathrm{E}-01$ & $3.38 \mathrm{E}-02$ \\
\hline & 102 & 26.595 & $8.91 \mathrm{E}+07$ & $7.37 \mathrm{E}+07$ & $2.35 \mathrm{E}-05$ & $1.94 \mathrm{E}-05$ & $2.05 \mathrm{E}-06$ \\
\hline & 125 & 23.922 & $2.88 \mathrm{E}+10$ & $3.36 \mathrm{E}+10$ & $8.65 \mathrm{E}-03$ & $1.01 \mathrm{E}-02$ & $6.81 \mathrm{E}-04$ \\
\hline & 134 & 23.535 & $8.51 \mathrm{E}+10$ & $1.01 \mathrm{E}+11$ & $2.52 \mathrm{E}-02$ & 2.99E-02 & $1.95 \mathrm{E}-03$ \\
\hline & 143 & 23.233 & $5.54 \mathrm{E}+10$ & $6.88 \mathrm{E}+10$ & 1.67E-02 & 2.07E-02 & $1.28 \mathrm{E}-03$ \\
\hline & 149 & 23.184 & $4.50 \mathrm{E}+10$ & $5.17 \mathrm{E}+10$ & $3.62 \mathrm{E}-03$ & $4.16 \mathrm{E}-03$ & $2.77 \mathrm{E}-04$ \\
\hline & 152 & 23.170 & $3.66 \mathrm{E}+11$ & $3.54 \mathrm{E}+11$ & 8.55E-02 & 8.27E-02 & $6.52 \mathrm{E}-03$ \\
\hline \multirow[t]{9}{*}{31} & 64 & 35.585 & $1.06 \mathrm{E}+11$ & $8.75 \mathrm{E}+10$ & $1.01 \mathrm{E}-01$ & $8.31 \mathrm{E}-02$ & $1.18 \mathrm{E}-02$ \\
\hline & 72 & 34.895 & $1.53 \mathrm{E}+10$ & $1.56 \mathrm{E}+10$ & $1.40 \mathrm{E}-02$ & $1.42 \mathrm{E}-02$ & $1.61 \mathrm{E}-03$ \\
\hline & 73 & 34.894 & $1.97 \mathrm{E}+11$ & $1.99 \mathrm{E}+11$ & $2.51 \mathrm{E}-01$ & $2.54 \mathrm{E}-01$ & $2.89 \mathrm{E}-02$ \\
\hline & 74 & 34.841 & $1.84 \mathrm{E}+11$ & $1.83 \mathrm{E}+11$ & $2.34 \mathrm{E}-01$ & $2.33 \mathrm{E}-01$ & $2.68 \mathrm{E}-02$ \\
\hline & 77 & 34.189 & $4.18 \mathrm{E}+09$ & $4.11 \mathrm{E}+09$ & $3.66 \mathrm{E}-03$ & $3.60 \mathrm{E}-03$ & $4.12 \mathrm{E}-04$ \\
\hline & 80 & 33.882 & $1.96 \mathrm{E}+09$ & $2.19 \mathrm{E}+09$ & $1.69 \mathrm{E}-03$ & $1.89 \mathrm{E}-03$ & $1.88 \mathrm{E}-04$ \\
\hline & 89 & 33.414 & $7.82 \mathrm{E}+09$ & $7.65 \mathrm{E}+09$ & $6.54 \mathrm{E}-03$ & $6.40 \mathrm{E}-03$ & $7.20 \mathrm{E}-04$ \\
\hline & 91 & 33.399 & $1.07 \mathrm{E}+11$ & $1.08 \mathrm{E}+11$ & $1.25 \mathrm{E}-01$ & $1.26 \mathrm{E}-01$ & $1.38 \mathrm{E}-02$ \\
\hline & 92 & 33.318 & $7.21 \mathrm{E}+08$ & $7.88 \mathrm{E}+08$ & $8.40 \mathrm{E}-04$ & $9.19 \mathrm{E}-04$ & $9.22 \mathrm{E}-05$ \\
\hline
\end{tabular}


Table 6. Cont.

\begin{tabular}{|c|c|c|c|c|c|c|c|}
\hline LL. & UL. & $\lambda$ & $A_{L}$ & $A_{V}$ & $f_{L}$ & $f_{V}$ & $S_{L}$ \\
\hline & 128 & 23.954 & $6.99 \mathrm{E}+10$ & $6.89 \mathrm{E}+10$ & $4.21 \mathrm{E}-02$ & $4.15 \mathrm{E}-02$ & $3.32 \mathrm{E}-03$ \\
\hline & 130 & 23.941 & $6.49 \mathrm{E}+10$ & $6.28 \mathrm{E}+10$ & 3.91E-02 & $3.78 \mathrm{E}-02$ & $3.08 \mathrm{E}-03$ \\
\hline & 150 & 23.229 & $3.73 \mathrm{E}+10$ & $3.81 \mathrm{E}+10$ & $2.11 \mathrm{E}-02$ & $2.16 \mathrm{E}-02$ & $1.62 \mathrm{E}-03$ \\
\hline & 174 & 11.482 & $3.91 \mathrm{E}+11$ & $3.38 \mathrm{E}+11$ & $5.42 \mathrm{E}-02$ & $4.68 \mathrm{E}-02$ & $2.05 \mathrm{E}-03$ \\
\hline \multirow[t]{23}{*}{32} & 42 & 716.687 & $6.30 \mathrm{E}+07$ & $5.90 \mathrm{E}+07$ & $2.43 \mathrm{E}-02$ & $2.27 \mathrm{E}-02$ & $5.72 \mathrm{E}-02$ \\
\hline & 57 & 40.507 & $9.53 \mathrm{E}+07$ & $9.76 \mathrm{E}+07$ & $1.17 \mathrm{E}-04$ & $1.20 \mathrm{E}-04$ & $1.56 \mathrm{E}-05$ \\
\hline & 58 & 40.413 & $2.24 \mathrm{E}+09$ & $2.35 \mathrm{E}+09$ & $3.85 \mathrm{E}-03$ & $4.03 \mathrm{E}-03$ & $5.12 \mathrm{E}-04$ \\
\hline & 59 & 40.312 & $8.43 \mathrm{E}+07$ & $8.20 \mathrm{E}+07$ & $1.44 \mathrm{E}-04$ & $1.40 \mathrm{E}-04$ & $1.91 \mathrm{E}-05$ \\
\hline & 68 & 34.998 & $2.62 \mathrm{E}+09$ & $2.50 \mathrm{E}+09$ & $3.37 \mathrm{E}-03$ & $3.21 \mathrm{E}-03$ & $3.88 \mathrm{E}-04$ \\
\hline & 71 & 34.921 & $2.37 \mathrm{E}+11$ & $2.15 E+11$ & $2.16 \mathrm{E}-01$ & $1.96 \mathrm{E}-01$ & $2.49 \mathrm{E}-02$ \\
\hline & 83 & 33.591 & $6.46 \mathrm{E}+11$ & $6.47 \mathrm{E}+11$ & $7.65 \mathrm{E}-01$ & $7.66 \mathrm{E}-01$ & $8.46 \mathrm{E}-02$ \\
\hline & 85 & 33.561 & $1.19 \mathrm{E}+11$ & $1.35 \mathrm{E}+11$ & $1.00 \mathrm{E}-01$ & $1.14 \mathrm{E}-01$ & $1.11 \mathrm{E}-02$ \\
\hline & 95 & 33.274 & $2.83 \mathrm{E}+10$ & $2.83 \mathrm{E}+10$ & $3.29 \mathrm{E}-02$ & $3.29 \mathrm{E}-02$ & $3.60 \mathrm{E}-03$ \\
\hline & 110 & 26.476 & $2.57 \mathrm{E}+07$ & $2.88 \mathrm{E}+07$ & $1.35 \mathrm{E}-05$ & $1.52 \mathrm{E}-05$ & $1.18 \mathrm{E}-06$ \\
\hline & 111 & 26.435 & $6.25 \mathrm{E}+08$ & $7.36 \mathrm{E}+08$ & $4.58 \mathrm{E}-04$ & $5.40 \mathrm{E}-04$ & $3.99 \mathrm{E}-05$ \\
\hline & 112 & 26.408 & $1.09 \mathrm{E}+07$ & $1.10 \mathrm{E}+07$ & 7.94E-06 & 8.04E-06 & $6.90 \mathrm{E}-07$ \\
\hline & 131 & 23.928 & $1.65 \mathrm{E}+06$ & $1.99 \mathrm{E}+06$ & $9.90 \mathrm{E}-07$ & $1.20 \mathrm{E}-06$ & $7.80 \mathrm{E}-08$ \\
\hline & 140 & 23.333 & $8.37 \mathrm{E}+09$ & $6.94 \mathrm{E}+09$ & $3.41 \mathrm{E}-03$ & $2.83 \mathrm{E}-03$ & $2.62 \mathrm{E}-04$ \\
\hline & 144 & 23.292 & $6.72 \mathrm{E}+10$ & $7.02 \mathrm{E}+10$ & $2.73 \mathrm{E}-02$ & $2.85 \mathrm{E}-02$ & $2.10 \mathrm{E}-03$ \\
\hline & 146 & 23.282 & $3.57 \mathrm{E}+11$ & $3.32 \mathrm{E}+11$ & 2.03E-01 & $1.89 \mathrm{E}-01$ & $1.55 \mathrm{E}-02$ \\
\hline & 153 & 23.234 & $9.26 \mathrm{E}+06$ & $8.02 \mathrm{E}+06$ & $3.75 \mathrm{E}-06$ & $3.24 \mathrm{E}-06$ & $2.87 \mathrm{E}-07$ \\
\hline & 157 & 23.219 & $1.83 \mathrm{E}+08$ & $1.62 \mathrm{E}+08$ & $1.04 \mathrm{E}-04$ & $9.18 \mathrm{E}-05$ & 7.93E-06 \\
\hline & 159 & 23.207 & $1.93 \mathrm{E}+10$ & $1.74 \mathrm{E}+10$ & $1.09 \mathrm{E}-02$ & $9.86 \mathrm{E}-03$ & 8.33E-04 \\
\hline & 161 & 23.202 & $6.16 \mathrm{E}+08$ & $5.70 \mathrm{E}+08$ & $3.48 \mathrm{E}-04$ & $3.22 \mathrm{E}-04$ & $2.66 \mathrm{E}-05$ \\
\hline & 169 & 11.672 & $1.38 \mathrm{E}+12$ & $1.33 \mathrm{E}+12$ & $1.41 \mathrm{E}-01$ & $1.36 \mathrm{E}-01$ & $5.42 \mathrm{E}-03$ \\
\hline & 174 & 11.392 & $2.68 \mathrm{E}+10$ & $2.75 \mathrm{E}+10$ & $2.61 \mathrm{E}-03$ & $2.68 \mathrm{E}-03$ & $9.79 \mathrm{E}-05$ \\
\hline & 179 & 11.356 & $1.09 \mathrm{E}+12$ & $1.10 \mathrm{E}+12$ & $1.48 \mathrm{E}-01$ & $1.49 \mathrm{E}-01$ & $5.53 \mathrm{E}-03$ \\
\hline \multirow[t]{12}{*}{33} & 64 & 35.677 & $5.71 \mathrm{E}+09$ & $6.14 \mathrm{E}+09$ & $5.45 \mathrm{E}-03$ & $5.86 \mathrm{E}-03$ & $6.40 \mathrm{E}-04$ \\
\hline & 65 & 35.660 & $1.24 \mathrm{E}+11$ & $1.03 \mathrm{E}+11$ & 7.12E-02 & $5.87 \mathrm{E}-02$ & $8.36 \mathrm{E}-03$ \\
\hline & 72 & 34.983 & $2.19 \mathrm{E}+11$ & $2.23 \mathrm{E}+11$ & $2.01 \mathrm{E}-01$ & $2.04 \mathrm{E}-01$ & $2.31 \mathrm{E}-02$ \\
\hline & 73 & 34.983 & $1.12 \mathrm{E}+10$ & $1.15 \mathrm{E}+10$ & $1.43 \mathrm{E}-02$ & $1.48 \mathrm{E}-02$ & $1.65 \mathrm{E}-03$ \\
\hline & 74 & 34.929 & $3.53 \mathrm{E}+12$ & $3.53 \mathrm{E}+12$ & $4.52 \mathrm{E}+00$ & $4.52 \mathrm{E}+00$ & $5.20 \mathrm{E}-01$ \\
\hline & 77 & 34.274 & $2.89 \mathrm{E}+09$ & $2.45 \mathrm{E}+09$ & $2.54 \mathrm{E}-03$ & $2.16 \mathrm{E}-03$ & $2.87 \mathrm{E}-04$ \\
\hline & 79 & 34.185 & $2.53 \mathrm{E}+09$ & $2.75 \mathrm{E}+09$ & $1.33 \mathrm{E}-03$ & $1.45 \mathrm{E}-03$ & $1.49 \mathrm{E}-04$ \\
\hline & 80 & 33.966 & $4.30 \mathrm{E}+09$ & $5.22 \mathrm{E}+09$ & $3.72 \mathrm{E}-03$ & $4.52 \mathrm{E}-03$ & $4.16 \mathrm{E}-04$ \\
\hline & 89 & 33.496 & $1.62 \mathrm{E}+10$ & $1.69 \mathrm{E}+10$ & $1.36 \mathrm{E}-02$ & $1.42 \mathrm{E}-02$ & $1.50 \mathrm{E}-03$ \\
\hline & 91 & 33.481 & $1.61 \mathrm{E}+11$ & $1.61 \mathrm{E}+11$ & $1.89 \mathrm{E}-01$ & $1.89 \mathrm{E}-01$ & $2.08 \mathrm{E}-02$ \\
\hline & 93 & 33.380 & $5.17 \mathrm{E}+07$ & $6.30 \mathrm{E}+07$ & $2.59 \mathrm{E}-05$ & $3.16 \mathrm{E}-05$ & $2.85 \mathrm{E}-06$ \\
\hline & 94 & 33.370 & $4.85 \mathrm{E}+10$ & $4.80 \mathrm{E}+10$ & 4.05E-02 & 4.00E-02 & $4.45 \mathrm{E}-03$ \\
\hline
\end{tabular}


Table 6. Cont.

\begin{tabular}{|c|c|c|c|c|c|c|c|}
\hline LL. & UL. & $\lambda$ & $A_{L}$ & $A_{V}$ & $f_{L}$ & $f_{V}$ & $S_{L}$ \\
\hline & 103 & 26.620 & $5.34 \mathrm{E}+08$ & $4.65 \mathrm{E}+08$ & $2.83 \mathrm{E}-04$ & $2.47 \mathrm{E}-04$ & $2.48 \mathrm{E}-05$ \\
\hline & 106 & 26.603 & $6.45 \mathrm{E}+07$ & $6.52 \mathrm{E}+07$ & $2.05 \mathrm{E}-05$ & $2.08 \mathrm{E}-05$ & $1.80 \mathrm{E}-06$ \\
\hline & 118 & 24.237 & $5.26 \mathrm{E}+09$ & $5.51 \mathrm{E}+09$ & $1.30 \mathrm{E}-03$ & $1.37 \mathrm{E}-03$ & $1.01 \mathrm{E}-04$ \\
\hline & 120 & 24.155 & $7.81 \mathrm{E}+10$ & $7.77 \mathrm{E}+10$ & $3.37 \mathrm{E}-02$ & $3.35 \mathrm{E}-02$ & $2.67 \mathrm{E}-03$ \\
\hline & 121 & 24.155 & $2.08 \mathrm{E}+06$ & $2.54 \mathrm{E}+07$ & $1.69 \mathrm{E}-07$ & $2.06 \mathrm{E}-06$ & $3.87 \mathrm{E}-08$ \\
\hline & 136 & 23.485 & $2.93 \mathrm{E}+09$ & $2.41 \mathrm{E}+09$ & $1.20 \mathrm{E}-03$ & $9.89 \mathrm{E}-04$ & $9.29 \mathrm{E}-05$ \\
\hline \multirow[t]{22}{*}{34} & 51 & 41.788 & $2.44 \mathrm{E}+09$ & $2.78 \mathrm{E}+09$ & $3.19 \mathrm{E}-03$ & 3.63E-03 & 4.39E-04 \\
\hline & 52 & 41.737 & $2.76 \mathrm{E}+08$ & $2.39 \mathrm{E}+08$ & $1.88 \mathrm{E}-04$ & $1.63 \mathrm{E}-04$ & $2.58 \mathrm{E}-05$ \\
\hline & 57 & 40.701 & $2.49 \mathrm{E}+06$ & $2.31 \mathrm{E}+06$ & $3.09 \mathrm{E}-06$ & 2.87E-06 & $4.13 \mathrm{E}-07$ \\
\hline & 62 & 36.681 & $1.13 \mathrm{E}+10$ & $1.68 \mathrm{E}+10$ & $2.28 \mathrm{E}-03$ & 3.38E-03 & $2.75 \mathrm{E}-04$ \\
\hline & 66 & 35.463 & $4.26 \mathrm{E}+08$ & $3.90 \mathrm{E}+08$ & 4.01E-04 & $3.68 \mathrm{E}-04$ & $4.68 \mathrm{E}-05$ \\
\hline & 71 & 35.065 & $5.42 \mathrm{E}+10$ & $5.63 \mathrm{E}+10$ & $5.00 \mathrm{E}-02$ & $5.18 \mathrm{E}-02$ & $5.77 \mathrm{E}-03$ \\
\hline & 75 & 34.995 & $6.66 \mathrm{E}+09$ & $5.52 \mathrm{E}+09$ & $3.04 \mathrm{E}-03$ & $2.52 \mathrm{E}-03$ & $3.50 \mathrm{E}-04$ \\
\hline & 84 & 33.709 & $1.34 \mathrm{E}+12$ & $1.31 \mathrm{E}+12$ & $6.71 \mathrm{E}-01$ & $6.56 \mathrm{E}-01$ & 7.44E-02 \\
\hline & 85 & 33.693 & $7.75 \mathrm{E}+11$ & $8.13 \mathrm{E}+11$ & $6.60 \mathrm{E}-01$ & $6.92 \mathrm{E}-01$ & 7.32E-02 \\
\hline & 95 & 33.418 & $1.50 \mathrm{E}+11$ & $1.34 \mathrm{E}+11$ & $6.72 \mathrm{E}-02$ & $6.00 \mathrm{E}-02$ & 7.39E-03 \\
\hline & 100 & 26.798 & $3.48 \mathrm{E}+09$ & $3.66 \mathrm{E}+09$ & $3.75 \mathrm{E}-04$ & $3.94 \mathrm{E}-04$ & $3.31 \mathrm{E}-05$ \\
\hline & 101 & 26.780 & $4.24 \mathrm{E}+09$ & $3.24 \mathrm{E}+09$ & $2.28 \mathrm{E}-03$ & $1.74 \mathrm{E}-03$ & $2.01 \mathrm{E}-04$ \\
\hline & 110 & 26.559 & $3.83 \mathrm{E}+05$ & $4.00 \mathrm{E}+05$ & 2.03E-07 & $2.11 \mathrm{E}-07$ & $1.77 \mathrm{E}-08$ \\
\hline & 122 & 24.129 & $1.27 \mathrm{E}+09$ & $1.11 \mathrm{E}+09$ & $5.56 \mathrm{E}-04$ & 4.83E-04 & $4.41 \mathrm{E}-05$ \\
\hline & 143 & 23.364 & $6.71 \mathrm{E}+11$ & $6.91 \mathrm{E}+11$ & $1.70 \mathrm{E}-01$ & $1.75 \mathrm{E}-01$ & $1.30 \mathrm{E}-02$ \\
\hline & 144 & 23.356 & $4.13 \mathrm{E}+11$ & $4.14 \mathrm{E}+11$ & $1.69 \mathrm{E}-01$ & $1.69 \mathrm{E}-01$ & $1.30 \mathrm{E}-02$ \\
\hline & 149 & 23.314 & $1.03 \mathrm{E}+12$ & $1.03 \mathrm{E}+12$ & $8.41 \mathrm{E}-02$ & $8.38 \mathrm{E}-02$ & $6.45 \mathrm{E}-03$ \\
\hline & 152 & 23.300 & $8.03 E+10$ & $7.41 \mathrm{E}+10$ & $1.81 \mathrm{E}-02$ & $1.67 \mathrm{E}-02$ & $1.39 \mathrm{E}-03$ \\
\hline & 153 & 23.298 & $1.25 \mathrm{E}+07$ & $1.06 \mathrm{E}+07$ & $5.08 \mathrm{E}-06$ & $4.31 \mathrm{E}-06$ & $3.90 \mathrm{E}-07$ \\
\hline & 167 & 11.728 & $7.39 \mathrm{E}+11$ & $7.23 E+11$ & $1.52 \mathrm{E}-02$ & $1.49 \mathrm{E}-02$ & $5.89 \mathrm{E}-04$ \\
\hline & 168 & 11.725 & $4.19 \mathrm{E}+11$ & $5.34 \mathrm{E}+11$ & $3.30 \mathrm{E}-02$ & $4.21 \mathrm{E}-02$ & $1.27 \mathrm{E}-03$ \\
\hline & 178 & 11.407 & $1.56 \mathrm{E}+11$ & $1.44 \mathrm{E}+11$ & $1.53 \mathrm{E}-02$ & $1.40 \mathrm{E}-02$ & $5.73 \mathrm{E}-04$ \\
\hline \multirow[t]{11}{*}{35} & 38 & 1222.229 & $1.14 \mathrm{E}+07$ & $1.40 \mathrm{E}+07$ & $1.28 \mathrm{E}-02$ & $1.57 \mathrm{E}-02$ & $5.15 \mathrm{E}-02$ \\
\hline & 45 & 515.564 & $1.27 \mathrm{E}+08$ & $1.26 \mathrm{E}+08$ & $3.55 \mathrm{E}-02$ & $3.51 \mathrm{E}-02$ & $6.02 \mathrm{E}-02$ \\
\hline & 57 & 40.721 & $3.96 \mathrm{E}+08$ & $4.09 \mathrm{E}+08$ & 4.92E-04 & $5.08 \mathrm{E}-04$ & $6.60 \mathrm{E}-05$ \\
\hline & 58 & 40.626 & $4.64 \mathrm{E}+09$ & $4.70 \mathrm{E}+09$ & $8.04 \mathrm{E}-03$ & $8.14 \mathrm{E}-03$ & $1.08 \mathrm{E}-03$ \\
\hline & 59 & 40.523 & $1.10 \mathrm{E}+10$ & $1.09 \mathrm{E}+10$ & $1.90 \mathrm{E}-02$ & $1.88 \mathrm{E}-02$ & $2.53 \mathrm{E}-03$ \\
\hline & 61 & 36.716 & $4.37 \mathrm{E}+10$ & $5.77 \mathrm{E}+10$ & $3.20 \mathrm{E}-03$ & $4.23 \mathrm{E}-03$ & $3.50 \mathrm{E}-02$ \\
\hline & 66 & 35.478 & $3.21 \mathrm{E}+10$ & $2.82 \mathrm{E}+10$ & $3.03 \mathrm{E}-02$ & $2.66 \mathrm{E}-02$ & $3.53 \mathrm{E}-03$ \\
\hline & 67 & 35.271 & $1.35 \mathrm{E}+11$ & $1.09 \mathrm{E}+11$ & $1.26 \mathrm{E}-01$ & $1.02 \mathrm{E}-01$ & $1.47 \mathrm{E}-02$ \\
\hline & 68 & 35.158 & $1.04 \mathrm{E}+11$ & $8.59 \mathrm{E}+10$ & $1.35 \mathrm{E}-01$ & $1.11 \mathrm{E}-01$ & $1.56 \mathrm{E}-02$ \\
\hline & 71 & 35.080 & $1.19 \mathrm{E}+11$ & $1.09 \mathrm{E}+11$ & $1.10 \mathrm{E}-01$ & $1.00 \mathrm{E}-01$ & $1.26 \mathrm{E}-02$ \\
\hline & 75 & 35.010 & $3.33 \mathrm{E}+10$ & $3.92 \mathrm{E}+10$ & $2.11 \mathrm{E}-03$ & $2.49 \mathrm{E}-03$ & $2.16 \mathrm{E}-02$ \\
\hline
\end{tabular}


Table 6. Cont.

\begin{tabular}{|c|c|c|c|c|c|c|c|}
\hline LL. & UL. & $\lambda$ & $A_{L}$ & $A_{V}$ & $f_{L}$ & $f_{V}$ & $S_{L}$ \\
\hline & 81 & 33.893 & $3.04 \mathrm{E}+11$ & $3.00 \mathrm{E}+11$ & $2.62 \mathrm{E}-01$ & $2.59 \mathrm{E}-01$ & 2.92E-02 \\
\hline & 84 & 33.723 & $3.81 \mathrm{E}+11$ & $3.83 \mathrm{E}+11$ & $2.16 \mathrm{E}-02$ & $2.17 \mathrm{E}-02$ & $1.96 \mathrm{E}-01$ \\
\hline & 85 & 33.707 & $6.15 E+11$ & $6.13 E+11$ & $5.24 \mathrm{E}-01$ & $5.22 \mathrm{E}-01$ & $5.81 \mathrm{E}-02$ \\
\hline & 83 & 33.738 & $1.26 \mathrm{E}+12$ & $1.22 \mathrm{E}+12$ & $1.51 \mathrm{E}+00$ & $1.46 \mathrm{E}+00$ & $1.67 \mathrm{E}-01$ \\
\hline & 96 & 33.419 & $9.74 \mathrm{E}+10$ & $1.01 \mathrm{E}+11$ & $1.14 \mathrm{E}-01$ & $1.18 \mathrm{E}-01$ & $1.26 \mathrm{E}-02$ \\
\hline & 110 & 26.567 & $9.61 \mathrm{E}+07$ & $1.14 \mathrm{E}+08$ & 5.09E-05 & $6.04 \mathrm{E}-05$ & 4.45E-06 \\
\hline & 111 & 26.526 & $9.01 \mathrm{E}+08$ & $1.03 \mathrm{E}+09$ & $6.65 \mathrm{E}-04$ & 7.61E-04 & $5.81 \mathrm{E}-05$ \\
\hline & 112 & 26.499 & $2.10 \mathrm{E}+09$ & $2.16 \mathrm{E}+09$ & $1.55 \mathrm{E}-03$ & $1.59 \mathrm{E}-03$ & $1.35 \mathrm{E}-04$ \\
\hline & 129 & 24.040 & $8.70 \mathrm{E}+10$ & $6.97 \mathrm{E}+10$ & $5.28 \mathrm{E}-02$ & 4.23E-02 & 4.18E-03 \\
\hline & 131 & 24.002 & $3.08 \mathrm{E}+08$ & $2.54 \mathrm{E}+08$ & $1.86 \mathrm{E}-04$ & $1.53 \mathrm{E}-04$ & $1.47 \mathrm{E}-05$ \\
\hline & 141 & 23.404 & $1.64 \mathrm{E}+11$ & $1.49 \mathrm{E}+11$ & $6.75 \mathrm{E}-02$ & $6.10 \mathrm{E}-02$ & $5.20 \mathrm{E}-03$ \\
\hline & 143 & 23.370 & $1.92 \mathrm{E}+11$ & $1.99 \mathrm{E}+11$ & $3.63 \mathrm{E}-03$ & $3.77 \mathrm{E}-03$ & $4.89 \mathrm{E}-02$ \\
\hline & 146 & 23.352 & $6.54 \mathrm{E}+11$ & $6.12 \mathrm{E}+11$ & $3.74 \mathrm{E}-01$ & $3.50 \mathrm{E}-01$ & $2.88 \mathrm{E}-02$ \\
\hline & 152 & 23.306 & $1.09 \mathrm{E}+08$ & $2.67 \mathrm{E}+07$ & $2.04 \mathrm{E}-06$ & $5.01 \mathrm{E}-07$ & $6.53 \mathrm{E}-06$ \\
\hline & 157 & 23.290 & $3.54 \mathrm{E}+07$ & $3.37 \mathrm{E}+07$ & $2.02 \mathrm{E}-05$ & $1.92 \mathrm{E}-05$ & $1.55 \mathrm{E}-06$ \\
\hline & 159 & 23.277 & $3.89 \mathrm{E}+10$ & $3.83 \mathrm{E}+10$ & $2.21 \mathrm{E}-02$ & $2.18 \mathrm{E}-02$ & $1.69 \mathrm{E}-03$ \\
\hline & 161 & 23.272 & $5.76 \mathrm{E}+09$ & $5.54 \mathrm{E}+09$ & $3.27 \mathrm{E}-03$ & $3.15 \mathrm{E}-03$ & $2.51 \mathrm{E}-04$ \\
\hline & 168 & 11.727 & $5.01 \mathrm{E}+11$ & $4.39 \mathrm{E}+11$ & $1.20 \mathrm{E}-03$ & $1.05 \mathrm{E}-03$ & $2.72 \mathrm{E}-02$ \\
\hline & 179 & 11.373 & $5.10 \mathrm{E}+12$ & $4.65 \mathrm{E}+12$ & $6.92 \mathrm{E}-01$ & $6.31 \mathrm{E}-01$ & $2.59 \mathrm{E}-02$ \\
\hline \multirow[t]{19}{*}{36} & 54 & 41.358 & $1.29 \mathrm{E}+09$ & $1.09 \mathrm{E}+09$ & $8.40 \mathrm{E}-04$ & $7.10 \mathrm{E}-04$ & $1.14 \mathrm{E}-04$ \\
\hline & 63 & 36.262 & $3.17 \mathrm{E}+10$ & $3.81 \mathrm{E}+10$ & $2.25 \mathrm{E}-02$ & $2.70 \mathrm{E}-02$ & 2.69E-03 \\
\hline & 64 & 35.908 & $2.33 \mathrm{E}+08$ & $1.96 \mathrm{E}+08$ & $2.66 \mathrm{E}-05$ & $2.24 \mathrm{E}-05$ & $1.90 \mathrm{E}-04$ \\
\hline & 65 & 35.891 & $1.18 \mathrm{E}+10$ & $1.53 \mathrm{E}+10$ & $8.88 \mathrm{E}-03$ & $1.15 \mathrm{E}-02$ & $1.05 \mathrm{E}-03$ \\
\hline & 72 & 35.206 & $3.17 \mathrm{E}+12$ & $3.16 \mathrm{E}+12$ & $3.42 \mathrm{E}-01$ & $3.40 \mathrm{E}-01$ & $2.94 \mathrm{E}+00$ \\
\hline & 76 & 34.540 & $3.90 \mathrm{E}+09$ & $4.39 \mathrm{E}+09$ & $2.35 \mathrm{E}-03$ & $2.65 \mathrm{E}-03$ & $2.68 \mathrm{E}-04$ \\
\hline & 77 & 34.487 & $3.52 \mathrm{E}+09$ & $2.86 \mathrm{E}+09$ & $3.56 \mathrm{E}-04$ & 2.89E-04 & $2.55 \mathrm{E}-03$ \\
\hline & 80 & 34.176 & $3.23 \mathrm{E}+09$ & $2.69 \mathrm{E}+09$ & $3.19 \mathrm{E}-04$ & $2.65 \mathrm{E}-04$ & $2.36 \mathrm{E}-03$ \\
\hline & 82 & 33.880 & $5.18 \mathrm{E}+09$ & $3.94 \mathrm{E}+09$ & 8.91E-04 & $6.78 \mathrm{E}-04$ & 9.94E-05 \\
\hline & 89 & 33.700 & $2.64 \mathrm{E}+10$ & $2.59 \mathrm{E}+10$ & $2.49 \mathrm{E}-03$ & $2.45 \mathrm{E}-03$ & $2.21 \mathrm{E}-02$ \\
\hline & 93 & 33.582 & $8.91 \mathrm{E}+09$ & $9.52 \mathrm{E}+09$ & $4.83 \mathrm{E}-03$ & $5.16 \mathrm{E}-03$ & 5.34E-04 \\
\hline & 94 & 33.573 & $8.77 \mathrm{E}+10$ & $8.82 \mathrm{E}+10$ & 8.19E-03 & 8.23E-03 & $7.45 \mathrm{E}-02$ \\
\hline & 127 & 24.110 & $1.03 \mathrm{E}+12$ & $1.05 \mathrm{E}+12$ & $3.56 \mathrm{E}-02$ & $3.64 \mathrm{E}-02$ & $4.59 \mathrm{E}-01$ \\
\hline & 136 & 23.585 & $3.68 \mathrm{E}+08$ & $3.88 \mathrm{E}+08$ & $1.19 \mathrm{E}-05$ & $1.26 \mathrm{E}-05$ & $1.62 \mathrm{E}-04$ \\
\hline & 147 & 23.394 & $1.29 \mathrm{E}+10$ & $1.34 \mathrm{E}+10$ & $4.08 \mathrm{E}-04$ & 4.24E-04 & $5.51 \mathrm{E}-03$ \\
\hline & 151 & 23.364 & $3.46 \mathrm{E}+10$ & $3.51 \mathrm{E}+10$ & $1.09 \mathrm{E}-03$ & $1.10 \mathrm{E}-03$ & $1.44 \mathrm{E}-02$ \\
\hline & 154 & 23.358 & $3.12 \mathrm{E}+09$ & $3.35 \mathrm{E}+09$ & $8.23 \mathrm{E}-04$ & $8.84 \mathrm{E}-04$ & $6.33 \mathrm{E}-05$ \\
\hline & 171 & 11.572 & $2.78 \mathrm{E}+10$ & $3.39 \mathrm{E}+10$ & $1.06 \mathrm{E}-04$ & $1.30 \mathrm{E}-04$ & $3.41 \mathrm{E}-03$ \\
\hline & 172 & 11.537 & $3.26 \mathrm{E}+11$ & $3.49 \mathrm{E}+11$ & $2.09 \mathrm{E}-02$ & $2.24 \mathrm{E}-02$ & 7.94E-04 \\
\hline 37 & 58 & 41.700 & $1.58 \mathrm{E}+09$ & $1.59 \mathrm{E}+09$ & $2.88 \mathrm{E}-03$ & $2.89 \mathrm{E}-03$ & $3.96 \mathrm{E}-04$ \\
\hline
\end{tabular}


Table 6. Cont.

\begin{tabular}{|c|c|c|c|c|c|c|c|}
\hline LL. & UL. & $\lambda$ & $A_{L}$ & $A_{V}$ & $f_{L}$ & $f_{V}$ & $S_{L}$ \\
\hline & 59 & 41.592 & $2.59 \mathrm{E}+10$ & $2.58 \mathrm{E}+10$ & 4.70E-02 & $4.68 \mathrm{E}-02$ & $6.43 \mathrm{E}-03$ \\
\hline & 66 & 36.295 & $1.22 \mathrm{E}+10$ & $1.10 \mathrm{E}+10$ & $1.20 \mathrm{E}-02$ & 1.09E-02 & $1.43 \mathrm{E}-03$ \\
\hline & 68 & 35.959 & $8.63 \mathrm{E}+09$ & $7.74 \mathrm{E}+09$ & $1.17 \mathrm{E}-02$ & $1.05 \mathrm{E}-02$ & $1.39 \mathrm{E}-03$ \\
\hline & 71 & 35.878 & $9.72 \mathrm{E}+10$ & $8.02 \mathrm{E}+10$ & 9.37E-02 & 7.74E-02 & $1.11 \mathrm{E}-02$ \\
\hline & 75 & 35.804 & $3.06 \mathrm{E}+11$ & $3.64 \mathrm{E}+11$ & $2.08 \mathrm{E}-02$ & $2.47 \mathrm{E}-02$ & $2.10 \mathrm{E}-01$ \\
\hline & 81 & 34.637 & $1.96 \mathrm{E}+11$ & $1.71 \mathrm{E}+11$ & $1.77 \mathrm{E}-01$ & $1.54 \mathrm{E}-01$ & $2.01 \mathrm{E}-02$ \\
\hline & 83 & 34.476 & $4.36 \mathrm{E}+10$ & $3.90 \mathrm{E}+10$ & 5.44E-02 & $4.86 \mathrm{E}-02$ & $6.17 \mathrm{E}-03$ \\
\hline & 84 & 34.459 & $6.23 E+10$ & $6.85 \mathrm{E}+10$ & $3.78 \mathrm{E}-03$ & $4.15 \mathrm{E}-03$ & $3.66 \mathrm{E}-02$ \\
\hline & 85 & 34.444 & $4.16 \mathrm{E}+11$ & $3.60 \mathrm{E}+11$ & $3.70 \mathrm{E}-01$ & $3.20 \mathrm{E}-01$ & 4.19E-02 \\
\hline & 95 & 34.155 & $4.42 \mathrm{E}+10$ & $3.96 \mathrm{E}+10$ & $2.61 \mathrm{E}-03$ & $2.33 \mathrm{E}-03$ & $2.08 \mathrm{E}-02$ \\
\hline & 96 & 34.142 & $1.75 \mathrm{E}+12$ & $1.68 \mathrm{E}+12$ & $2.14 \mathrm{E}+00$ & $2.06 \mathrm{E}+00$ & $2.40 \mathrm{E}-01$ \\
\hline & 111 & 26.980 & $1.57 \mathrm{E}+08$ & $1.79 \mathrm{E}+08$ & $1.20 \mathrm{E}-04$ & $1.37 \mathrm{E}-04$ & $1.07 \mathrm{E}-05$ \\
\hline & 112 & 26.952 & $3.94 \mathrm{E}+09$ & $4.21 \mathrm{E}+09$ & $3.00 \mathrm{E}-03$ & $3.21 \mathrm{E}-03$ & $2.67 \mathrm{E}-04$ \\
\hline & 129 & 24.412 & $1.72 \mathrm{E}+10$ & $1.48 \mathrm{E}+10$ & $1.07 \mathrm{E}-02$ & $9.26 \mathrm{E}-03$ & 8.64E-04 \\
\hline & 131 & 24.373 & $1.18 \mathrm{E}+08$ & $9.88 \mathrm{E}+07$ & $7.35 \mathrm{E}-05$ & $6.16 \mathrm{E}-05$ & $5.90 \mathrm{E}-06$ \\
\hline & 141 & 23.756 & $9.92 \mathrm{E}+10$ & $8.26 \mathrm{E}+10$ & $4.20 \mathrm{E}-02$ & 3.49E-02 & $3.28 \mathrm{E}-03$ \\
\hline & 143 & 23.722 & $2.99 \mathrm{E}+10$ & $3.48 \mathrm{E}+10$ & 5.91E-04 & $6.87 \mathrm{E}-04$ & $8.80 \mathrm{E}-03$ \\
\hline & 146 & 23.703 & $1.77 \mathrm{E}+10$ & $1.54 \mathrm{E}+10$ & $1.05 \mathrm{E}-02$ & $9.06 \mathrm{E}-03$ & $8.16 \mathrm{E}-04$ \\
\hline & 157 & 23.639 & $9.06 \mathrm{E}+08$ & $8.11 \mathrm{E}+08$ & 5.31E-04 & 4.75E-04 & 4.13E-05 \\
\hline & 159 & 23.625 & $8.84 \mathrm{E}+11$ & $8.12 \mathrm{E}+11$ & $5.18 \mathrm{E}-01$ & 4.75E-01 & 4.03E-02 \\
\hline & 161 & 23.621 & $6.88 \mathrm{E}+10$ & $6.30 \mathrm{E}+10$ & 4.03E-02 & $3.69 \mathrm{E}-02$ & $3.13 \mathrm{E}-03$ \\
\hline \multirow[t]{15}{*}{38} & 72 & 36.116 & $2.82 \mathrm{E}+10$ & $2.77 \mathrm{E}+10$ & $2.75 \mathrm{E}-02$ & $2.71 \mathrm{E}-02$ & $3.27 \mathrm{E}-03$ \\
\hline & 73 & 36.115 & $1.47 \mathrm{E}+10$ & $1.44 \mathrm{E}+10$ & $2.02 \mathrm{E}-02$ & $1.98 \mathrm{E}-02$ & $2.40 \mathrm{E}-03$ \\
\hline & 74 & 36.058 & $1.87 \mathrm{E}+11$ & $1.85 \mathrm{E}+11$ & $2.56 \mathrm{E}-01$ & $2.53 \mathrm{E}-01$ & $3.03 \mathrm{E}-02$ \\
\hline & 76 & 35.415 & $8.01 E+10$ & $7.09 \mathrm{E}+10$ & $4.52 \mathrm{E}-02$ & $4.00 \mathrm{E}-02$ & $5.27 \mathrm{E}-03$ \\
\hline & 77 & 35.360 & $1.75 E+10$ & $1.49 \mathrm{E}+10$ & 1.64E-02 & $1.40 \mathrm{E}-02$ & $1.91 \mathrm{E}-03$ \\
\hline & 79 & 35.265 & $9.27 \mathrm{E}+09$ & $9.93 \mathrm{E}+09$ & $5.18 \mathrm{E}-03$ & $5.56 \mathrm{E}-03$ & $6.02 \mathrm{E}-04$ \\
\hline & 80 & 35.032 & $8.73 \mathrm{E}+06$ & $1.06 \mathrm{E}+07$ & 8.03E-06 & 9.71E-06 & $9.26 \mathrm{E}-07$ \\
\hline & 89 & 34.532 & $5.29 \mathrm{E}+11$ & $5.33 E+11$ & 4.73E-01 & 4.77E-01 & $5.37 \mathrm{E}-02$ \\
\hline & 91 & 34.516 & $2.87 \mathrm{E}+12$ & $2.88 \mathrm{E}+12$ & $3.59 \mathrm{E}+00$ & $3.60 \mathrm{E}+00$ & $4.08 \mathrm{E}-01$ \\
\hline & 93 & 34.409 & $3.30 \mathrm{E}+10$ & $3.25 \mathrm{E}+10$ & $1.76 \mathrm{E}-02$ & $1.73 \mathrm{E}-02$ & $1.99 \mathrm{E}-03$ \\
\hline & 94 & 34.399 & $1.87 \mathrm{E}+11$ & $1.88 \mathrm{E}+11$ & 1.66E-01 & $1.67 \mathrm{E}-01$ & $1.88 \mathrm{E}-02$ \\
\hline & 120 & 24.533 & $5.10 \mathrm{E}+09$ & $4.83 \mathrm{E}+09$ & $2.30 \mathrm{E}-03$ & $2.18 \mathrm{E}-03$ & $1.86 \mathrm{E}-04$ \\
\hline & 138 & 23.952 & $6.32 \mathrm{E}+09$ & $4.76 \mathrm{E}+09$ & $1.63 \mathrm{E}-03$ & $1.23 \mathrm{E}-03$ & $1.29 \mathrm{E}-04$ \\
\hline & 154 & 23.755 & $1.29 \mathrm{E}+10$ & $1.08 \mathrm{E}+10$ & $3.27 \mathrm{E}-03$ & $2.75 \mathrm{E}-03$ & $2.56 \mathrm{E}-04$ \\
\hline & 172 & 11.633 & $4.93 E+11$ & $4.54 \mathrm{E}+11$ & $3.00 \mathrm{E}-02$ & 2.77E-02 & $1.15 \mathrm{E}-03$ \\
\hline \multirow[t]{3}{*}{39} & 91 & 34.627 & $2.24 \mathrm{E}+10$ & $2.26 \mathrm{E}+10$ & $2.81 \mathrm{E}-02$ & $2.85 \mathrm{E}-02$ & $3.21 \mathrm{E}-03$ \\
\hline & 92 & 34.540 & $3.52 \mathrm{E}+10$ & $3.46 \mathrm{E}+10$ & 4.41E-02 & 4.33E-02 & $5.02 \mathrm{E}-03$ \\
\hline & 150 & 23.817 & $7.70 \mathrm{E}+09$ & $7.56 \mathrm{E}+09$ & $4.58 \mathrm{E}-03$ & $4.50 \mathrm{E}-03$ & $3.59 \mathrm{E}-04$ \\
\hline
\end{tabular}


Table 6. Cont.

\begin{tabular}{|c|c|c|c|c|c|c|c|}
\hline LL. & UL. & $\lambda$ & $A_{L}$ & $A_{V}$ & $f_{L}$ & $f_{V}$ & $S_{L}$ \\
\hline & 156 & 23.797 & $1.40 \mathrm{E}+10$ & $1.20 \mathrm{E}+10$ & 8.34E-03 & $7.15 \mathrm{E}-03$ & $6.53 \mathrm{E}-04$ \\
\hline & 174 & 11.624 & $1.35 \mathrm{E}+12$ & $1.04 \mathrm{E}+12$ & $1.92 \mathrm{E}-01$ & $1.47 \mathrm{E}-01$ & $7.35 \mathrm{E}-03$ \\
\hline \multirow[t]{13}{*}{40} & 73 & 36.523 & $1.79 \mathrm{E}+10$ & $1.78 \mathrm{E}+10$ & $2.51 \mathrm{E}-02$ & $2.50 \mathrm{E}-02$ & $3.02 \mathrm{E}-03$ \\
\hline & 74 & 36.464 & $3.41 \mathrm{E}+09$ & $3.38 \mathrm{E}+09$ & 4.75E-03 & 4.72E-03 & $5.71 \mathrm{E}-04$ \\
\hline & 77 & 35.751 & $7.07 \mathrm{E}+10$ & $5.89 \mathrm{E}+10$ & $6.77 \mathrm{E}-02$ & $5.64 \mathrm{E}-02$ & 7.97E-03 \\
\hline & 80 & 35.416 & $8.14 \mathrm{E}+09$ & $7.45 \mathrm{E}+09$ & $7.65 \mathrm{E}-03$ & $7.00 \mathrm{E}-03$ & 8.92E-04 \\
\hline & 89 & 34.905 & $1.21 \mathrm{E}+11$ & $1.21 \mathrm{E}+11$ & $1.11 \mathrm{E}-01$ & $1.10 \mathrm{E}-01$ & $1.27 \mathrm{E}-02$ \\
\hline & 91 & 34.889 & $4.86 \mathrm{E}+11$ & $4.88 \mathrm{E}+11$ & $6.21 \mathrm{E}-01$ & $6.23 \mathrm{E}-01$ & $7.14 \mathrm{E}-02$ \\
\hline & 92 & 34.800 & $6.56 \mathrm{E}+11$ & $6.56 \mathrm{E}+11$ & 8.34E-01 & $8.34 \mathrm{E}-01$ & $9.55 \mathrm{E}-02$ \\
\hline & 94 & 34.769 & $2.97 \mathrm{E}+10$ & $2.98 \mathrm{E}+10$ & 2.69E-02 & $2.70 \mathrm{E}-02$ & $3.08 \mathrm{E}-03$ \\
\hline & 128 & 24.711 & $5.28 \mathrm{E}+09$ & $5.04 \mathrm{E}+09$ & $3.38 \mathrm{E}-03$ & $3.23 \mathrm{E}-03$ & $2.75 \mathrm{E}-04$ \\
\hline & 130 & 24.697 & $2.87 \mathrm{E}+09$ & $2.77 \mathrm{E}+09$ & $1.84 \mathrm{E}-03$ & $1.77 \mathrm{E}-03$ & $1.50 \mathrm{E}-04$ \\
\hline & 150 & 23.940 & $1.61 \mathrm{E}+11$ & $1.59 \mathrm{E}+11$ & $9.71 \mathrm{E}-02$ & $9.54 \mathrm{E}-02$ & 7.65E-03 \\
\hline & 156 & 23.920 & $2.35 \mathrm{E}+11$ & $2.29 \mathrm{E}+11$ & $1.41 \mathrm{E}-01$ & $1.37 \mathrm{E}-01$ & $1.11 \mathrm{E}-02$ \\
\hline & 174 & 11.653 & $4.37 \mathrm{E}+11$ & $3.81 \mathrm{E}+11$ & $6.22 \mathrm{E}-02$ & $5.44 \mathrm{E}-02$ & $2.39 \mathrm{E}-03$ \\
\hline \multirow[t]{15}{*}{41} & 64 & 37.470 & $4.00 \mathrm{E}+09$ & $5.21 \mathrm{E}+09$ & $5.20 \mathrm{E}-04$ & $6.77 \mathrm{E}-04$ & $5.49 \mathrm{E}-03$ \\
\hline & 65 & 37.452 & $1.50 \mathrm{E}+09$ & $1.38 \mathrm{E}+09$ & 8.69E-04 & 7.99E-04 & $1.07 \mathrm{E}-04$ \\
\hline & 72 & 36.707 & $4.50 \mathrm{E}+09$ & $4.57 \mathrm{E}+09$ & $5.49 \mathrm{E}-04$ & $5.58 \mathrm{E}-04$ & 4.61E-03 \\
\hline & 76 & 35.983 & $5.15 \mathrm{E}+09$ & $6.52 \mathrm{E}+09$ & $3.79 \mathrm{E}-03$ & $4.80 \mathrm{E}-03$ & 4.49E-04 \\
\hline & 77 & 35.926 & $1.80 \mathrm{E}+10$ & $2.24 \mathrm{E}+10$ & $2.06 \mathrm{E}-03$ & $2.57 \mathrm{E}-03$ & $2.17 \mathrm{E}-02$ \\
\hline & 79 & 35.829 & $5.11 \mathrm{E}+10$ & $6.10 \mathrm{E}+10$ & $3.52 \mathrm{E}-02$ & $4.20 \mathrm{E}-02$ & $4.16 \mathrm{E}-03$ \\
\hline & 82 & 35.267 & $4.00 \mathrm{E}+09$ & $3.39 \mathrm{E}+09$ & $7.46 \mathrm{E}-04$ & $6.33 \mathrm{E}-04$ & $8.66 \mathrm{E}-05$ \\
\hline & 89 & 35.072 & $1.86 \mathrm{E}+12$ & $1.85 \mathrm{E}+12$ & $1.98 \mathrm{E}-01$ & $1.97 \mathrm{E}-01$ & $1.70 \mathrm{E}+00$ \\
\hline & 93 & 34.945 & $1.83 \mathrm{E}+12$ & $1.84 \mathrm{E}+12$ & $1.01 \mathrm{E}+00$ & $1.02 \mathrm{E}+00$ & $1.16 \mathrm{E}-01$ \\
\hline & 94 & 34.935 & $3.44 \mathrm{E}+11$ & $3.42 \mathrm{E}+11$ & $3.62 \mathrm{E}-02$ & $3.60 \mathrm{E}-02$ & $3.13 \mathrm{E}-01$ \\
\hline & 127 & 24.804 & $4.62 \mathrm{E}+09$ & $4.58 \mathrm{E}+09$ & $1.74 \mathrm{E}-04$ & $1.72 \mathrm{E}-04$ & $2.11 \mathrm{E}-03$ \\
\hline & 147 & 24.047 & $6.28 \mathrm{E}+11$ & $6.39 \mathrm{E}+11$ & $2.16 \mathrm{E}-02$ & $2.19 \mathrm{E}-02$ & $2.77 \mathrm{E}-01$ \\
\hline & 151 & 24.016 & $1.01 \mathrm{E}+11$ & $1.01 \mathrm{E}+11$ & $3.44 \mathrm{E}-03$ & $3.46 \mathrm{E}-03$ & 4.37E-02 \\
\hline & 154 & 24.009 & $6.22 \mathrm{E}+11$ & $6.34 \mathrm{E}+11$ & $1.64 \mathrm{E}-01$ & $1.67 \mathrm{E}-01$ & $1.30 \mathrm{E}-02$ \\
\hline & 172 & 11.693 & $3.24 \mathrm{E}+11$ & $4.02 \mathrm{E}+11$ & $2.47 \mathrm{E}-02$ & $3.06 \mathrm{E}-02$ & $9.51 \mathrm{E}-04$ \\
\hline \multirow[t]{9}{*}{42} & 65 & 37.455 & $1.34 \mathrm{E}+09$ & $1.37 \mathrm{E}+09$ & $8.48 \mathrm{E}-04$ & 8.63E-04 & $1.05 \mathrm{E}-04$ \\
\hline & 72 & 36.709 & $2.04 \mathrm{E}+09$ & $1.91 \mathrm{E}+09$ & $2.06 \mathrm{E}-03$ & $1.93 \mathrm{E}-03$ & $2.49 \mathrm{E}-04$ \\
\hline & 73 & 36.708 & $1.31 \mathrm{E}+10$ & $1.30 \mathrm{E}+10$ & $1.85 \mathrm{E}-02$ & $1.83 \mathrm{E}-02$ & $2.24 \mathrm{E}-03$ \\
\hline & 74 & 36.649 & $7.08 \mathrm{E}+09$ & $6.85 E+09$ & $9.98 \mathrm{E}-03$ & $9.65 \mathrm{E}-03$ & $1.20 \mathrm{E}-03$ \\
\hline & 78 & 35.832 & $3.03 \mathrm{E}+10$ & $2.47 \mathrm{E}+10$ & $4.08 \mathrm{E}-02$ & $3.33 \mathrm{E}-02$ & $4.82 \mathrm{E}-03$ \\
\hline & 79 & 35.831 & $9.15 \mathrm{E}+10$ & $7.82 \mathrm{E}+10$ & $5.28 \mathrm{E}-02$ & $4.52 \mathrm{E}-02$ & $6.23 \mathrm{E}-03$ \\
\hline & 80 & 35.590 & $9.78 \mathrm{E}+09$ & $8.20 \mathrm{E}+09$ & $9.29 \mathrm{E}-03$ & $7.78 \mathrm{E}-03$ & $1.09 \mathrm{E}-03$ \\
\hline & 89 & 35.074 & $8.44 \mathrm{E}+11$ & $8.46 \mathrm{E}+11$ & $7.78 \mathrm{E}-01$ & $7.80 \mathrm{E}-01$ & $8.98 \mathrm{E}-02$ \\
\hline & 91 & 35.058 & $2.08 \mathrm{E}+11$ & $2.08 \mathrm{E}+11$ & $2.68 \mathrm{E}-01$ & $2.69 \mathrm{E}-01$ & $3.09 \mathrm{E}-02$ \\
\hline
\end{tabular}


Table 6. Cont.

\begin{tabular}{|c|c|c|c|c|c|c|c|}
\hline LL. & UL. & $\lambda$ & $A_{L}$ & $A_{V}$ & $f_{L}$ & $f_{V}$ & $S_{L}$ \\
\hline & 93 & 34.947 & $2.32 \mathrm{E}+11$ & $2.33 \mathrm{E}+11$ & $1.28 \mathrm{E}-01$ & $1.28 \mathrm{E}-01$ & $1.47 \mathrm{E}-02$ \\
\hline & 94 & 34.937 & $6.18 \mathrm{E}+11$ & $6.20 \mathrm{E}+11$ & $5.65 \mathrm{E}-01$ & $5.67 \mathrm{E}-01$ & $6.50 \mathrm{E}-02$ \\
\hline & 106 & 27.589 & $6.34 \mathrm{E}+07$ & $6.37 \mathrm{E}+07$ & $2.17 \mathrm{E}-05$ & $2.18 \mathrm{E}-05$ & $1.97 \mathrm{E}-06$ \\
\hline & 118 & 24.965 & $5.35 \mathrm{E}+08$ & $4.37 \mathrm{E}+08$ & $1.50 \mathrm{E}-04$ & $1.23 \mathrm{E}-04$ & $1.23 \mathrm{E}-05$ \\
\hline & 120 & 24.805 & $2.01 \mathrm{E}+09$ & $1.76 \mathrm{E}+09$ & $9.26 \mathrm{E}-04$ & $8.11 \mathrm{E}-04$ & $7.56 \mathrm{E}-05$ \\
\hline & 154 & 24.010 & $8.20 \mathrm{E}+10$ & $7.86 \mathrm{E}+10$ & $2.13 \mathrm{E}-02$ & $2.04 \mathrm{E}-02$ & $1.68 \mathrm{E}-03$ \\
\hline & 172 & 11.694 & $1.72 \mathrm{E}+11$ & $1.51 \mathrm{E}+11$ & $1.06 \mathrm{E}-02$ & $9.28 \mathrm{E}-03$ & 4.08E-04 \\
\hline \multirow[t]{10}{*}{43} & 63 & 38.020 & $5.45 \mathrm{E}+08$ & $5.92 \mathrm{E}+08$ & $1.64 \mathrm{E}-02$ & $1.78 \mathrm{E}-02$ & $1.39 \mathrm{E}-02$ \\
\hline & 65 & 37.613 & $3.17 \mathrm{E}+07$ & $1.99 \mathrm{E}+07$ & 8.27E-04 & $5.18 \mathrm{E}-04$ & $6.55 \mathrm{E}-04$ \\
\hline & 76 & 36.132 & $1.58 \mathrm{E}+09$ & $1.91 \mathrm{E}+09$ & 2.59E-02 & $3.12 \mathrm{E}-02$ & $1.62 \mathrm{E}-02$ \\
\hline & 79 & 35.976 & $1.01 \mathrm{E}+09$ & $1.37 \mathrm{E}+09$ & $1.58 \mathrm{E}-02$ & $2.14 \mathrm{E}-02$ & $9.71 \mathrm{E}-03$ \\
\hline & 93 & 35.085 & $4.56 \mathrm{E}+07$ & $3.74 \mathrm{E}+07$ & $5.57 \mathrm{E}-04$ & 4.57E-04 & $3.02 \mathrm{E}-04$ \\
\hline & 97 & 28.129 & $2.48 \mathrm{E}+10$ & $2.87 \mathrm{E}+10$ & $6.49 \mathrm{E}-02$ & $7.50 \mathrm{E}-02$ & $1.63 \mathrm{E}-02$ \\
\hline & 104 & 27.675 & $2.30 \mathrm{E}+11$ & $2.32 \mathrm{E}+11$ & $5.52 \mathrm{E}-01$ & $5.55 \mathrm{E}-01$ & $1.32 \mathrm{E}-01$ \\
\hline & 138 & 24.278 & $3.37 \mathrm{E}+08$ & $4.02 \mathrm{E}+08$ & 4.15E-04 & $4.95 \mathrm{E}-04$ & $7.16 \mathrm{E}-05$ \\
\hline & 154 & 24.076 & $4.38 \mathrm{E}+08$ & $3.07 \mathrm{E}+08$ & 4.97E-05 & $3.48 \mathrm{E}-05$ & $2.60 \mathrm{E}-06$ \\
\hline & 172 & 11.709 & $3.03 \mathrm{E}+09$ & $2.53 \mathrm{E}+09$ & $3.40 \mathrm{E}-04$ & $2.84 \mathrm{E}-04$ & $1.77 \mathrm{E}-05$ \\
\hline \multirow[t]{8}{*}{44} & 73 & 37.052 & $2.52 \mathrm{E}+10$ & $2.51 \mathrm{E}+10$ & $3.74 \mathrm{E}-02$ & $3.73 \mathrm{E}-02$ & 4.63E-03 \\
\hline & 74 & 36.993 & $2.34 \mathrm{E}+10$ & $2.36 \mathrm{E}+10$ & $3.47 \mathrm{E}-02$ & $3.49 \mathrm{E}-02$ & $4.29 \mathrm{E}-03$ \\
\hline & 84 & 35.543 & $1.39 \mathrm{E}+10$ & $1.33 \mathrm{E}+10$ & $7.88 \mathrm{E}-03$ & $7.58 \mathrm{E}-03$ & $9.22 \mathrm{E}-04$ \\
\hline & 95 & 35.219 & $9.70 \mathrm{E}+11$ & $9.39 \mathrm{E}+11$ & $5.41 \mathrm{E}-01$ & $5.24 \mathrm{E}-01$ & $6.28 \mathrm{E}-02$ \\
\hline & 128 & 25.217 & $7.11 \mathrm{E}+09$ & $6.92 \mathrm{E}+09$ & $4.75 \mathrm{E}-03$ & 4.62E-03 & $3.94 \mathrm{E}-04$ \\
\hline & 130 & 25.202 & $5.51 \mathrm{E}+09$ & $5.42 \mathrm{E}+09$ & $3.67 \mathrm{E}-03$ & $3.62 \mathrm{E}-03$ & $3.05 \mathrm{E}-04$ \\
\hline & 143 & 24.230 & $8.31 \mathrm{E}+09$ & $6.80 \mathrm{E}+09$ & 2.19E-03 & $1.80 \mathrm{E}-03$ & $1.75 \mathrm{E}-04$ \\
\hline & 152 & 24.161 & $5.36 \mathrm{E}+11$ & $4.47 \mathrm{E}+11$ & $1.41 \mathrm{E}-01$ & $1.17 \mathrm{E}-01$ & $1.12 \mathrm{E}-02$ \\
\hline 45 & 94 & 35.779 & $1.41 \mathrm{E}+10$ & $1.33 \mathrm{E}+10$ & $1.35 \mathrm{E}-02$ & $1.27 \mathrm{E}-02$ & $1.60 \mathrm{E}-03$ \\
\hline \multirow[t]{10}{*}{46} & 72 & 37.683 & $9.88 \mathrm{E}+10$ & $9.83 E+10$ & $1.30 \mathrm{E}-02$ & $1.30 \mathrm{E}-02$ & $1.05 \mathrm{E}-01$ \\
\hline & 80 & 36.506 & $1.24 \mathrm{E}+10$ & $1.47 \mathrm{E}+10$ & $1.49 \mathrm{E}-03$ & $1.77 \mathrm{E}-03$ & $1.47 \mathrm{E}-02$ \\
\hline & 82 & 36.168 & $1.12 \mathrm{E}+11$ & $7.97 \mathrm{E}+10$ & $2.20 \mathrm{E}-02$ & $1.56 \mathrm{E}-02$ & $2.62 \mathrm{E}-03$ \\
\hline & 89 & 35.963 & $5.34 \mathrm{E}+11$ & $5.30 \mathrm{E}+11$ & $6.13 \mathrm{E}-02$ & $6.08 \mathrm{E}-02$ & $5.13 \mathrm{E}-01$ \\
\hline & 93 & 35.829 & $1.06 \mathrm{E}+10$ & $1.04 \mathrm{E}+10$ & $5.98 \mathrm{E}-03$ & $5.87 \mathrm{E}-03$ & 7.05E-04 \\
\hline & 94 & 35.818 & $2.57 \mathrm{E}+12$ & $2.55 \mathrm{E}+12$ & 2.91E-01 & $2.90 \mathrm{E}-01$ & $2.46 \mathrm{E}+00$ \\
\hline & 127 & 25.247 & $4.04 \mathrm{E}+10$ & $4.24 \mathrm{E}+10$ & $1.60 \mathrm{E}-03$ & $1.69 \mathrm{E}-03$ & $2.03 \mathrm{E}-02$ \\
\hline & 147 & 24.463 & $1.43 \mathrm{E}+11$ & $1.47 \mathrm{E}+11$ & $5.15 \mathrm{E}-03$ & $5.29 \mathrm{E}-03$ & $6.57 \mathrm{E}-02$ \\
\hline & 151 & 24.430 & $8.60 \mathrm{E}+11$ & $8.62 \mathrm{E}+11$ & $3.10 \mathrm{E}-02$ & $3.10 \mathrm{E}-02$ & $3.86 \mathrm{E}-01$ \\
\hline & 154 & 24.424 & $3.44 \mathrm{E}+09$ & $3.35 \mathrm{E}+09$ & 8.99E-04 & $8.75 \mathrm{E}-04$ & $7.23 \mathrm{E}-05$ \\
\hline
\end{tabular}


Table 6. Cont.

\begin{tabular}{|c|c|c|c|c|c|c|c|}
\hline LL. & UL. & $\lambda$ & $A_{L}$ & $A_{V}$ & $f_{L}$ & $f_{V}$ & $S_{L}$ \\
\hline & 171 & 11.827 & $4.90 \mathrm{E}+10$ & $5.80 \mathrm{E}+10$ & $2.00 \mathrm{E}-04$ & $2.37 \mathrm{E}-04$ & $6.08 \mathrm{E}-03$ \\
\hline & 172 & 11.791 & $2.75 \mathrm{E}+10$ & $2.73 E+10$ & $1.71 \mathrm{E}-03$ & $1.70 \mathrm{E}-03$ & $6.63 \mathrm{E}-05$ \\
\hline \multirow[t]{24}{*}{47} & 49 & 1504.950 & $1.36 \mathrm{E}+08$ & $1.49 \mathrm{E}+08$ & $1.52 \mathrm{E}-01$ & $1.67 \mathrm{E}-01$ & 7.54E-01 \\
\hline & 51 & 1231.018 & $3.37 \mathrm{E}+08$ & $2.94 \mathrm{E}+08$ & $3.83 \mathrm{E}-01$ & $3.34 \mathrm{E}-01$ & $1.55 \mathrm{E}+00$ \\
\hline & 52 & 1188.480 & $6.69 \mathrm{E}+07$ & $6.95 \mathrm{E}+07$ & $4.41 \mathrm{E}-02$ & 4.58E-02 & $1.73 \mathrm{E}-01$ \\
\hline & 57 & 688.833 & $2.79 \mathrm{E}+02$ & $2.16 \mathrm{E}+02$ & $9.94 \mathrm{E}-08$ & 7.69E-08 & $2.25 \mathrm{E}-07$ \\
\hline & 61 & 242.111 & $1.11 \mathrm{E}+09$ & $1.38 \mathrm{E}+09$ & $3.64 \mathrm{E}-02$ & $4.53 \mathrm{E}-02$ & $2.90 \mathrm{E}-02$ \\
\hline & 62 & 241.304 & $1.24 \mathrm{E}+09$ & $1.90 \mathrm{E}+09$ & $1.08 \mathrm{E}-02$ & $1.66 \mathrm{E}-02$ & 8.57E-03 \\
\hline & 66 & 196.828 & $8.76 \mathrm{E}+06$ & $9.12 \mathrm{E}+06$ & $2.54 \mathrm{E}-04$ & $2.65 \mathrm{E}-04$ & $1.65 \mathrm{E}-04$ \\
\hline & 67 & 190.626 & $8.93 \mathrm{E}+08$ & 8.37E+08 & $2.43 \mathrm{E}-02$ & $2.28 \mathrm{E}-02$ & $1.53 \mathrm{E}-02$ \\
\hline & 71 & 185.158 & $3.32 \mathrm{E}+09$ & $2.78 \mathrm{E}+09$ & $8.52 \mathrm{E}-02$ & $7.15 \mathrm{E}-02$ & $5.20 \mathrm{E}-02$ \\
\hline & 75 & 183.220 & $1.13 \mathrm{E}+09$ & $1.22 \mathrm{E}+09$ & $1.84 \mathrm{E}-02$ & 1.99E-02 & $1.11 \mathrm{E}-02$ \\
\hline & 99 & 70.707 & $1.63 \mathrm{E}+11$ & $1.68 \mathrm{E}+11$ & $3.78 \mathrm{E}-01$ & $3.90 \mathrm{E}-01$ & $8.80 \mathrm{E}-02$ \\
\hline & 100 & 70.431 & $2.08 \mathrm{E}+11$ & $2.07 \mathrm{E}+11$ & $1.54 \mathrm{E}-01$ & $1.54 \mathrm{E}-01$ & $3.58 \mathrm{E}-02$ \\
\hline & 101 & 70.307 & $1.92 \mathrm{E}+11$ & $1.86 \mathrm{E}+11$ & 7.11E-01 & $6.91 \mathrm{E}-01$ & $1.65 \mathrm{E}-01$ \\
\hline & 102 & 70.211 & $2.75 \mathrm{E}+10$ & $2.84 \mathrm{E}+10$ & $6.29 \mathrm{E}-02$ & $6.50 \mathrm{E}-02$ & $1.45 \mathrm{E}-02$ \\
\hline & 110 & 68.800 & $8.82 \mathrm{E}+04$ & $8.94 \mathrm{E}+04$ & $3.13 \mathrm{E}-07$ & $3.17 \mathrm{E}-07$ & 7.09E-08 \\
\hline & 122 & 54.565 & $1.30 \mathrm{E}+07$ & $1.24 \mathrm{E}+07$ & $2.90 \mathrm{E}-05$ & $2.77 \mathrm{E}-05$ & $5.21 \mathrm{E}-06$ \\
\hline & 124 & 54.292 & $2.93 \mathrm{E}+08$ & $2.73 \mathrm{E}+08$ & $6.47 \mathrm{E}-04$ & $6.04 \mathrm{E}-04$ & $1.16 \mathrm{E}-04$ \\
\hline & 125 & 54.217 & $1.04 \mathrm{E}+08$ & $1.04 \mathrm{E}+08$ & $1.37 \mathrm{E}-04$ & $1.37 \mathrm{E}-04$ & $2.45 \mathrm{E}-05$ \\
\hline & 141 & 50.962 & $1.15 \mathrm{E}+07$ & $1.11 \mathrm{E}+07$ & $2.23 \mathrm{E}-05$ & $2.16 \mathrm{E}-05$ & $3.75 \mathrm{E}-06$ \\
\hline & 143 & 50.804 & $2.42 \mathrm{E}+08$ & $2.51 \mathrm{E}+08$ & $2.91 \mathrm{E}-04$ & $3.02 \mathrm{E}-04$ & 4.87E-05 \\
\hline & 144 & 50.765 & $1.84 \mathrm{E}+08$ & $1.79 \mathrm{E}+08$ & $3.55 \mathrm{E}-04$ & $3.45 \mathrm{E}-04$ & $5.93 \mathrm{E}-05$ \\
\hline & 149 & 50.568 & $3.66 \mathrm{E}+08$ & $3.78 \mathrm{E}+08$ & $1.40 \mathrm{E}-04$ & $1.45 \mathrm{E}-04$ & $2.33 \mathrm{E}-05$ \\
\hline & 152 & 50.501 & $4.13 \mathrm{E}+07$ & $4.18 \mathrm{E}+07$ & $4.80 \mathrm{E}-05$ & $4.86 \mathrm{E}-05$ & 7.98E-06 \\
\hline & 153 & 50.493 & $4.32 \mathrm{E}+03$ & $4.54 \mathrm{E}+03$ & 8.26E-09 & 8.67E-09 & 1.37E-09 \\
\hline \multirow[t]{5}{*}{48} & 52 & 1801.170 & $8.38 \mathrm{E}+07$ & $7.43 \mathrm{E}+07$ & $1.22 \mathrm{E}-01$ & $1.08 \mathrm{E}-01$ & $7.25 \mathrm{E}-01$ \\
\hline & 75 & 193.360 & $4.23 \mathrm{E}+09$ & $3.51 \mathrm{E}+09$ & $7.11 \mathrm{E}-02$ & $5.90 \mathrm{E}-02$ & $4.53 \mathrm{E}-02$ \\
\hline & 99 & 72.168 & $3.21 \mathrm{E}+10$ & $3.00 \mathrm{E}+10$ & $7.51 \mathrm{E}-02$ & 7.02E-02 & $1.78 \mathrm{E}-02$ \\
\hline & 102 & 71.651 & $1.70 \mathrm{E}+11$ & $1.61 \mathrm{E}+11$ & 3.93E-01 & $3.71 \mathrm{E}-01$ & $9.26 \mathrm{E}-02$ \\
\hline & 152 & 51.242 & $2.48 \mathrm{E}+08$ & $2.69 \mathrm{E}+08$ & $2.93 \mathrm{E}-04$ & $3.18 \mathrm{E}-04$ & 4.94E-05 \\
\hline \multirow[t]{8}{*}{49} & 64 & 239.155 & $2.13 \mathrm{E}+08$ & $2.36 \mathrm{E}+08$ & $7.20 \mathrm{E}-03$ & 7.97E-03 & $1.01 \mathrm{E}-02$ \\
\hline & 72 & 211.113 & $1.05 \mathrm{E}+07$ & $9.29 \mathrm{E}+06$ & $2.45 \mathrm{E}-04$ & $2.16 \mathrm{E}-04$ & $3.11 \mathrm{E}-04$ \\
\hline & 77 & 187.659 & $3.36 \mathrm{E}+09$ & $3.89 \mathrm{E}+09$ & $5.48 \mathrm{E}-02$ & $6.34 \mathrm{E}-02$ & $1.03 \mathrm{E}-01$ \\
\hline & 82 & 170.982 & $1.01 \mathrm{E}+08$ & $7.05 \mathrm{E}+07$ & $4.43 \mathrm{E}-04$ & $3.09 \mathrm{E}-04$ & $2.49 \mathrm{E}-04$ \\
\hline & 89 & 166.487 & $6.64 \mathrm{E}+07$ & $6.16 \mathrm{E}+07$ & $7.56 \mathrm{E}-04$ & 7.02E-04 & $1.28 \mathrm{E}-03$ \\
\hline & 97 & 75.994 & $6.24 \mathrm{E}+10$ & $7.07 \mathrm{E}+10$ & $1.84 \mathrm{E}-01$ & $2.08 \mathrm{E}-01$ & $4.60 \mathrm{E}-02$ \\
\hline & 103 & 72.897 & $2.76 \mathrm{E}+11$ & $2.79 \mathrm{E}+11$ & $2.64 \mathrm{E}-01$ & $2.67 \mathrm{E}-01$ & $1.11 \mathrm{E}+00$ \\
\hline & 105 & 72.309 & $5.03 \mathrm{E}+10$ & $5.00 \mathrm{E}+10$ & 4.69E-02 & 4.67E-02 & $1.96 \mathrm{E}-01$ \\
\hline
\end{tabular}


Table 6. Cont.

\begin{tabular}{|c|c|c|c|c|c|c|c|}
\hline LL. & UL. & $\lambda$ & $A_{L}$ & $A_{V}$ & $f_{L}$ & $f_{V}$ & $S_{L}$ \\
\hline & 127 & 56.151 & $4.47 \mathrm{E}+08$ & $4.80 \mathrm{E}+08$ & $1.95 \mathrm{E}-04$ & $2.10 \mathrm{E}-04$ & $1.13 \mathrm{E}-03$ \\
\hline & 147 & 52.415 & $2.45 \mathrm{E}+08$ & $2.92 \mathrm{E}+08$ & $8.71 \mathrm{E}-05$ & $1.04 \mathrm{E}-04$ & $6.02 \mathrm{E}-04$ \\
\hline & 151 & 52.266 & $1.94 \mathrm{E}+07$ & $2.21 \mathrm{E}+07$ & $6.83 \mathrm{E}-06$ & $7.78 \mathrm{E}-06$ & $4.52 \mathrm{E}-05$ \\
\hline & 154 & 52.236 & $2.04 \mathrm{E}+08$ & $2.45 \mathrm{E}+08$ & $3.00 \mathrm{E}-04$ & $3.60 \mathrm{E}-04$ & $5.16 \mathrm{E}-05$ \\
\hline \multirow[t]{9}{*}{51} & 56 & 1694.597 & $2.67 \mathrm{E}+05$ & $2.42 \mathrm{E}+05$ & $5.75 \mathrm{E}-04$ & $5.20 \mathrm{E}-04$ & $3.21 \mathrm{E}-03$ \\
\hline & 80 & 183.648 & $1.92 \mathrm{E}+09$ & $1.54 \mathrm{E}+09$ & $4.85 \mathrm{E}-02$ & $3.89 \mathrm{E}-02$ & $2.93 \mathrm{E}-02$ \\
\hline & 89 & 170.689 & $1.35 \mathrm{E}+07$ & $1.48 \mathrm{E}+07$ & $2.94 \mathrm{E}-04$ & $3.23 \mathrm{E}-04$ & $1.65 \mathrm{E}-04$ \\
\hline & 97 & 76.858 & $1.51 \mathrm{E}+11$ & $1.32 \mathrm{E}+11$ & $4.01 \mathrm{E}-01$ & $3.52 \mathrm{E}-01$ & $1.02 \mathrm{E}-01$ \\
\hline & 103 & 73.691 & $1.04 \mathrm{E}+11$ & $1.03 \mathrm{E}+11$ & $4.23 \mathrm{E}-01$ & $4.18 \mathrm{E}-01$ & $1.03 \mathrm{E}-01$ \\
\hline & 104 & 73.561 & $1.18 \mathrm{E}+10$ & $1.17 \mathrm{E}+10$ & $2.88 \mathrm{E}-02$ & $2.86 \mathrm{E}-02$ & $6.98 \mathrm{E}-03$ \\
\hline & 105 & 73.090 & $1.00 \mathrm{E}+09$ & $9.70 \mathrm{E}+08$ & $4.01 \mathrm{E}-03$ & $3.89 \mathrm{E}-03$ & $9.64 \mathrm{E}-04$ \\
\hline & 120 & 57.523 & $6.65 \mathrm{E}+07$ & $5.76 \mathrm{E}+07$ & $1.60 \mathrm{E}-04$ & $1.39 \mathrm{E}-04$ & $2.98 \mathrm{E}-05$ \\
\hline & 138 & 53.622 & $2.06 \mathrm{E}+07$ & $1.72 \mathrm{E}+07$ & $2.56 \mathrm{E}-05$ & $2.14 \mathrm{E}-05$ & 4.44E-06 \\
\hline \multirow[t]{13}{*}{52} & 64 & 249.722 & $3.59 \mathrm{E}+08$ & $4.28 \mathrm{E}+08$ & $1.38 \mathrm{E}-02$ & $1.64 \mathrm{E}-02$ & $2.00 \mathrm{E}-02$ \\
\hline & 72 & 219.305 & $1.29 \mathrm{E}+07$ & $1.62 \mathrm{E}+07$ & $3.35 \mathrm{E}-04$ & $4.21 \mathrm{E}-04$ & $5.83 \mathrm{E}-04$ \\
\hline & 80 & 184.634 & $1.49 \mathrm{E}+09$ & $1.62 \mathrm{E}+09$ & $2.31 \mathrm{E}-02$ & $2.51 \mathrm{E}-02$ & $4.14 \mathrm{E}-02$ \\
\hline & 82 & 176.316 & $6.02 \mathrm{E}+09$ & $4.01 \mathrm{E}+09$ & $2.81 \mathrm{E}-02$ & $1.87 \mathrm{E}-02$ & $1.63 \mathrm{E}-02$ \\
\hline & 89 & 171.540 & $2.37 \mathrm{E}+07$ & $2.87 \mathrm{E}+07$ & $2.96 \mathrm{E}-04$ & $3.57 \mathrm{E}-04$ & $6.33 \mathrm{E}-04$ \\
\hline & 93 & 168.548 & $6.96 \mathrm{E}+06$ & $5.87 \mathrm{E}+06$ & $7.50 \mathrm{E}-05$ & $6.33 \mathrm{E}-05$ & $4.16 \mathrm{E}-05$ \\
\hline & 94 & 168.300 & $1.73 \mathrm{E}+08$ & $1.81 \mathrm{E}+08$ & $2.04 \mathrm{E}-03$ & $2.13 \mathrm{E}-03$ & $3.84 \mathrm{E}-03$ \\
\hline & 97 & 77.030 & $1.52 \mathrm{E}+10$ & $1.71 E+10$ & $4.55 \mathrm{E}-02$ & $5.12 \mathrm{E}-02$ & $1.15 \mathrm{E}-02$ \\
\hline & 103 & 73.849 & $3.28 \mathrm{E}+10$ & $3.35 \mathrm{E}+10$ & $3.26 \mathrm{E}-02$ & 3.33E-02 & $1.37 \mathrm{E}-01$ \\
\hline & 105 & 73.246 & $3.58 \mathrm{E}+11$ & $3.58 \mathrm{E}+11$ & $3.47 \mathrm{E}-01$ & $3.47 \mathrm{E}-01$ & $1.44 \mathrm{E}+00$ \\
\hline & 127 & 56.714 & $1.87 \mathrm{E}+08$ & $1.81 \mathrm{E}+08$ & $8.42 \mathrm{E}-05$ & $8.16 \mathrm{E}-05$ & 4.37E-04 \\
\hline & 147 & 52.905 & $8.96 \mathrm{E}+06$ & $1.04 \mathrm{E}+07$ & $3.27 \mathrm{E}-06$ & $3.81 \mathrm{E}-06$ & $2.19 \mathrm{E}-05$ \\
\hline & 154 & 52.723 & $4.82 \mathrm{E}+07$ & $5.56 \mathrm{E}+07$ & $6.95 \mathrm{E}-05$ & $8.02 \mathrm{E}-05$ & $1.21 \mathrm{E}-05$ \\
\hline \multirow[t]{12}{*}{53} & 67 & 244.871 & $5.59 \mathrm{E}+07$ & $5.14 \mathrm{E}+07$ & $2.51 \mathrm{E}-03$ & $2.31 \mathrm{E}-03$ & $2.02 \mathrm{E}-03$ \\
\hline & 68 & 239.492 & $3.60 \mathrm{E}+08$ & $2.96 \mathrm{E}+08$ & $2.17 \mathrm{E}-02$ & $1.78 \mathrm{E}-02$ & $1.71 \mathrm{E}-02$ \\
\hline & 96 & 176.811 & $6.76 \mathrm{E}+07$ & $8.48 \mathrm{E}+07$ & $2.22 \mathrm{E}-03$ & $2.78 \mathrm{E}-03$ & $1.29 \mathrm{E}-03$ \\
\hline & 99 & 77.038 & $4.11 \mathrm{E}+10$ & $5.47 \mathrm{E}+10$ & $2.78 \mathrm{E}-02$ & $3.70 \mathrm{E}-02$ & $1.46 \mathrm{E}-01$ \\
\hline & 102 & 76.449 & $3.53 \mathrm{E}+09$ & $4.37 \mathrm{E}+09$ & $2.33 \mathrm{E}-03$ & $2.89 \mathrm{E}-03$ & $1.15 \mathrm{E}-02$ \\
\hline & 110 & 74.779 & $1.15 \mathrm{E}+11$ & $1.14 \mathrm{E}+11$ & $4.81 \mathrm{E}-01$ & 4.79E-01 & 1.19E-01 \\
\hline & 111 & 74.455 & $6.76 \mathrm{E}+11$ & $6.71 \mathrm{E}+11$ & $3.93 \mathrm{E}+00$ & $3.90 \mathrm{E}+00$ & $9.63 \mathrm{E}-01$ \\
\hline & 112 & 74.240 & $6.30 \mathrm{E}+08$ & $6.08 \mathrm{E}+08$ & $3.64 \mathrm{E}-03$ & $3.52 \mathrm{E}-03$ & $8.90 \mathrm{E}-04$ \\
\hline & 125 & 57.863 & $7.30 \mathrm{E}+07$ & $7.73 \mathrm{E}+07$ & $2.09 \mathrm{E}-05$ & $2.22 \mathrm{E}-05$ & $1.16 \mathrm{E}-04$ \\
\hline & 131 & 57.488 & $1.05 \mathrm{E}+08$ & $8.83 \mathrm{E}+07$ & $3.64 \mathrm{E}-04$ & $3.06 \mathrm{E}-04$ & $6.89 \mathrm{E}-05$ \\
\hline & 146 & 53.895 & $1.01 \mathrm{E}+08$ & $1.16 \mathrm{E}+08$ & $3.08 \mathrm{E}-04$ & $3.53 \mathrm{E}-04$ & $5.47 \mathrm{E}-05$ \\
\hline & 157 & 53.562 & $7.16 \mathrm{E}+07$ & $6.23 \mathrm{E}+07$ & $2.16 \mathrm{E}-04$ & $1.88 \mathrm{E}-04$ & $3.80 \mathrm{E}-05$ \\
\hline
\end{tabular}


Table 6. Cont.

\begin{tabular}{|c|c|c|c|c|c|c|c|}
\hline LL. & UL. & $\lambda$ & $A_{L}$ & $A_{V}$ & $f_{L}$ & $f_{V}$ & $S_{L}$ \\
\hline & 179 & 15.708 & $1.01 \mathrm{E}+08$ & $9.31 \mathrm{E}+07$ & $2.63 \mathrm{E}-05$ & $2.41 \mathrm{E}-05$ & $1.36 \mathrm{E}-06$ \\
\hline \multirow[t]{12}{*}{54} & 62 & 336.919 & $7.47 \mathrm{E}+06$ & $9.72 \mathrm{E}+06$ & $1.27 \mathrm{E}-04$ & $1.65 \mathrm{E}-04$ & $1.41 \mathrm{E}-04$ \\
\hline & 66 & 256.115 & $5.65 \mathrm{E}+08$ & $5.38 \mathrm{E}+08$ & $2.78 \mathrm{E}-02$ & $2.64 \mathrm{E}-02$ & $2.34 \mathrm{E}-02$ \\
\hline & 81 & 191.453 & $1.12 \mathrm{E}+09$ & $1.01 \mathrm{E}+09$ & 3.07E-02 & $2.78 \mathrm{E}-02$ & $1.94 \mathrm{E}-02$ \\
\hline & 85 & 185.688 & $8.19 \mathrm{E}+08$ & $6.23 \mathrm{E}+08$ & $2.12 \mathrm{E}-02$ & $1.61 \mathrm{E}-02$ & $1.29 \mathrm{E}-02$ \\
\hline & 95 & 177.607 & $1.01 \mathrm{E}+08$ & $1.03 \mathrm{E}+08$ & $1.46 \mathrm{E}-03$ & $1.49 \mathrm{E}-03$ & $8.51 \mathrm{E}-04$ \\
\hline & 99 & 77.121 & $1.25 \mathrm{E}+10$ & $1.65 \mathrm{E}+10$ & $4.40 \mathrm{E}-02$ & $5.81 \mathrm{E}-02$ & $1.12 \mathrm{E}-02$ \\
\hline & 100 & 76.791 & $6.26 \mathrm{E}+10$ & $8.05 E+10$ & $5.54 \mathrm{E}-02$ & $7.11 \mathrm{E}-02$ & $1.40 \mathrm{E}-02$ \\
\hline & 101 & 76.644 & $7.55 \mathrm{E}+08$ & $5.69 \mathrm{E}+08$ & $3.32 \mathrm{E}-03$ & $2.51 \mathrm{E}-03$ & 8.39E-04 \\
\hline & 110 & 74.857 & $6.22 \mathrm{E}+11$ & $6.20 \mathrm{E}+11$ & $2.61 \mathrm{E}+00$ & $2.61 \mathrm{E}+00$ & $6.44 \mathrm{E}-01$ \\
\hline & 122 & 58.306 & $2.77 \mathrm{E}+08$ & $4.98 \mathrm{E}+08$ & $7.06 \mathrm{E}-04$ & $1.27 \mathrm{E}-03$ & $1.35 \mathrm{E}-04$ \\
\hline & 125 & 57.910 & $1.19 \mathrm{E}+08$ & $1.09 \mathrm{E}+08$ & $1.65 \mathrm{E}-04$ & $1.51 \mathrm{E}-04$ & $3.14 \mathrm{E}-05$ \\
\hline & 169 & 16.323 & $2.84 \mathrm{E}+08$ & $2.28 \mathrm{E}+08$ & $6.13 \mathrm{E}-04$ & $4.93 \mathrm{E}-04$ & $1.08 \mathrm{E}-04$ \\
\hline \multirow[t]{10}{*}{55} & 71 & 243.133 & $2.31 \mathrm{E}+08$ & $1.90 \mathrm{E}+08$ & $1.02 \mathrm{E}-02$ & $8.41 \mathrm{E}-03$ & $8.20 \mathrm{E}-03$ \\
\hline & 110 & 75.489 & $3.23 \mathrm{E}+09$ & $3.26 \mathrm{E}+09$ & $1.38 \mathrm{E}-02$ & $1.39 \mathrm{E}-02$ & $3.43 \mathrm{E}-03$ \\
\hline & 111 & 75.158 & $8.14 \mathrm{E}+10$ & $8.18 \mathrm{E}+10$ & 4.83E-01 & $4.85 \mathrm{E}-01$ & $1.19 \mathrm{E}-01$ \\
\hline & 112 & 74.940 & $1.35 \mathrm{E}+09$ & $1.32 \mathrm{E}+09$ & 7.93E-03 & $7.80 \mathrm{E}-03$ & $1.96 \mathrm{E}-03$ \\
\hline & 129 & 58.128 & $4.25 \mathrm{E}+06$ & $2.40 \mathrm{E}+06$ & $1.51 \mathrm{E}-05$ & $8.53 \mathrm{E}-06$ & $2.88 \mathrm{E}-06$ \\
\hline & 131 & 57.906 & $1.44 \mathrm{E}+07$ & $1.33 \mathrm{E}+07$ & $5.06 \mathrm{E}-05$ & 4.67E-05 & $9.65 \mathrm{E}-06$ \\
\hline & 153 & 54.005 & $1.37 \mathrm{E}+06$ & $1.13 \mathrm{E}+06$ & $3.01 \mathrm{E}-06$ & $2.47 \mathrm{E}-06$ & $5.34 \mathrm{E}-07$ \\
\hline & 159 & 53.856 & $5.36 \mathrm{E}+06$ & $5.51 \mathrm{E}+06$ & $1.63 \mathrm{E}-05$ & $1.68 \mathrm{E}-05$ & $2.89 \mathrm{E}-06$ \\
\hline & 161 & 53.833 & $1.62 \mathrm{E}+07$ & $2.12 \mathrm{E}+07$ & 4.92E-05 & $6.44 \mathrm{E}-05$ & 8.73E-06 \\
\hline & 179 & 15.739 & $2.33 \mathrm{E}+08$ & $2.45 \mathrm{E}+08$ & $6.06 \mathrm{E}-05$ & $6.38 \mathrm{E}-05$ & $3.14 \mathrm{E}-06$ \\
\hline \multirow[t]{8}{*}{56} & 68 & 254.120 & $1.82 \mathrm{E}+08$ & $1.90 \mathrm{E}+08$ & $1.23 \mathrm{E}-02$ & $1.29 \mathrm{E}-02$ & $1.03 \mathrm{E}-02$ \\
\hline & 96 & 184.659 & $1.72 \mathrm{E}+09$ & $1.85 \mathrm{E}+09$ & $6.15 \mathrm{E}-02$ & $6.62 \mathrm{E}-02$ & $3.74 \mathrm{E}-02$ \\
\hline & 101 & 77.997 & $1.04 \mathrm{E}+08$ & $8.35 \mathrm{E}+07$ & 4.73E-04 & $3.81 \mathrm{E}-04$ & $1.21 \mathrm{E}-04$ \\
\hline & 110 & 76.148 & $9.55 \mathrm{E}+08$ & $9.77 \mathrm{E}+08$ & $4.15 \mathrm{E}-03$ & $4.25 \mathrm{E}-03$ & $1.04 \mathrm{E}-03$ \\
\hline & 111 & 75.811 & $1.56 \mathrm{E}+09$ & $1.57 \mathrm{E}+09$ & $9.39 \mathrm{E}-03$ & $9.48 \mathrm{E}-03$ & $2.34 \mathrm{E}-03$ \\
\hline & 112 & 75.589 & $7.62 \mathrm{E}+11$ & $7.54 \mathrm{E}+11$ & $4.57 \mathrm{E}+00$ & $4.52 \mathrm{E}+00$ & $1.14 \mathrm{E}+00$ \\
\hline & 131 & 58.293 & $1.95 \mathrm{E}+06$ & $1.64 \mathrm{E}+06$ & $6.94 \mathrm{E}-06$ & $5.85 \mathrm{E}-06$ & $1.33 \mathrm{E}-06$ \\
\hline & 157 & 54.261 & $5.96 \mathrm{E}+05$ & $5.36 \mathrm{E}+05$ & $1.84 \mathrm{E}-06$ & $1.66 \mathrm{E}-06$ & $3.29 \mathrm{E}-07$ \\
\hline \multirow[t]{6}{*}{57} & 77 & 220.189 & $6.08 \mathrm{E}+06$ & 7.19E+06 & $2.21 \mathrm{E}-04$ & $2.61 \mathrm{E}-04$ & $1.60 \mathrm{E}-04$ \\
\hline & 78 & 216.621 & $2.21 \mathrm{E}+05$ & $2.72 \mathrm{E}+05$ & $1.09 \mathrm{E}-05$ & $1.34 \mathrm{E}-05$ & 7.77E-06 \\
\hline & 80 & 208.081 & $7.46 \mathrm{E}+06$ & $6.50 \mathrm{E}+06$ & $2.42 \mathrm{E}-04$ & $2.11 \mathrm{E}-04$ & $1.66 \mathrm{E}-04$ \\
\hline & 91 & 191.108 & $1.53 \mathrm{E}+09$ & $1.24 \mathrm{E}+09$ & $5.85 \mathrm{E}-02$ & $4.76 \mathrm{E}-02$ & $3.68 \mathrm{E}-02$ \\
\hline & 120 & 59.717 & $3.99 \mathrm{E}+07$ & $4.37 \mathrm{E}+07$ & $1.03 \mathrm{E}-04$ & $1.13 \mathrm{E}-04$ & $2.00 \mathrm{E}-05$ \\
\hline & 150 & 54.521 & $2.28 \mathrm{E}+08$ & $1.93 \mathrm{E}+08$ & $1.10 \mathrm{E}-03$ & $6.01 \mathrm{E}-04$ & $2.47 \mathrm{E}-04$ \\
\hline 58 & 73 & 256.883 & $5.41 \mathrm{E}+08$ & $6.44 \mathrm{E}+08$ & $3.75 \mathrm{E}-02$ & $4.46 \mathrm{E}-02$ & $3.17 \mathrm{E}-02$ \\
\hline
\end{tabular}


Table 6. Cont.

\begin{tabular}{|c|c|c|c|c|c|c|c|}
\hline LL. & UL. & $\lambda$ & $A_{L}$ & $A_{V}$ & $f_{L}$ & $f_{V}$ & $S_{L}$ \\
\hline & 74 & 254.025 & $1.26 \mathrm{E}+08$ & $1.53 \mathrm{E}+08$ & $8.56 \mathrm{E}-03$ & $1.04 \mathrm{E}-02$ & $7.16 \mathrm{E}-03$ \\
\hline & 113 & 76.179 & $9.39 \mathrm{E}+10$ & $9.35 \mathrm{E}+10$ & $5.72 \mathrm{E}-01$ & $5.69 \mathrm{E}-01$ & $1.43 \mathrm{E}-01$ \\
\hline \multirow[t]{5}{*}{59} & 64 & 305.390 & $3.01 \mathrm{E}+07$ & $2.79 \mathrm{E}+07$ & $2.11 \mathrm{E}-03$ & $1.95 \mathrm{E}-03$ & $2.12 \mathrm{E}-03$ \\
\hline & 73 & 261.050 & $1.88 \mathrm{E}+08$ & $1.84 \mathrm{E}+08$ & $1.35 \mathrm{E}-02$ & $1.32 \mathrm{E}-02$ & $1.16 \mathrm{E}-02$ \\
\hline & 74 & 258.099 & $3.56 \mathrm{E}+08$ & $3.70 \mathrm{E}+08$ & 2.49E-02 & $2.59 \mathrm{E}-02$ & $2.11 \mathrm{E}-02$ \\
\hline & 78 & 222.384 & $1.43 \mathrm{E}+06$ & $1.37 \mathrm{E}+06$ & $7.42 \mathrm{E}-05$ & 7.13E-05 & $5.43 \mathrm{E}-05$ \\
\hline & 113 & 76.541 & $3.40 \mathrm{E}+09$ & $3.38 \mathrm{E}+09$ & 2.09E-02 & $2.08 \mathrm{E}-02$ & $5.26 \mathrm{E}-03$ \\
\hline \multirow[t]{15}{*}{61} & 63 & 2252.530 & $3.18 \mathrm{E}+07$ & $3.52 \mathrm{E}+07$ & 8.04E-02 & $8.90 \mathrm{E}-02$ & $5.96 \mathrm{E}-01$ \\
\hline & 64 & 1397.564 & $1.93 \mathrm{E}+08$ & $2.43 \mathrm{E}+08$ & $1.30 \mathrm{E}+00$ & $1.64 \mathrm{E}+00$ & $3.55 \mathrm{E}-01$ \\
\hline & 65 & 1372.700 & $5.98 \mathrm{E}+07$ & $7.61 \mathrm{E}+07$ & $6.45 \mathrm{E}-02$ & $8.21 \mathrm{E}-02$ & $2.91 \mathrm{E}-01$ \\
\hline & 89 & 393.605 & $1.44 \mathrm{E}+06$ & $1.68 \mathrm{E}+06$ & $2.17 \mathrm{E}-04$ & $2.53 \mathrm{E}-04$ & $1.95 \mathrm{E}-04$ \\
\hline & 93 & 378.197 & $4.06 \mathrm{E}+06$ & $5.35 \mathrm{E}+06$ & $3.44 \mathrm{E}-04$ & $4.53 \mathrm{E}-04$ & $4.29 \mathrm{E}-04$ \\
\hline & 105 & 96.491 & $1.64 \mathrm{E}+09$ & $1.75 \mathrm{E}+09$ & $3.64 \mathrm{E}-03$ & $3.87 \mathrm{E}-03$ & $1.22 \mathrm{E}-02$ \\
\hline & 118 & 71.704 & $1.27 \mathrm{E}+11$ & $1.35 \mathrm{E}+11$ & $3.11 \mathrm{E}-01$ & $3.31 \mathrm{E}-01$ & $7.35 \mathrm{E}-02$ \\
\hline & 120 & 71.090 & $1.88 \mathrm{E}+11$ & $1.96 \mathrm{E}+11$ & $1.67 \mathrm{E}-01$ & $1.73 \mathrm{E}-01$ & $7.41 \mathrm{E}-01$ \\
\hline & 121 & 70.992 & $5.98 \mathrm{E}+10$ & $6.24 \mathrm{E}+10$ & $1.42 \mathrm{E}-01$ & $1.48 \mathrm{E}-01$ & $3.31 \mathrm{E}-02$ \\
\hline & 127 & 69.719 & $6.32 \mathrm{E}+08$ & $5.94 \mathrm{E}+08$ & $5.28 \mathrm{E}-04$ & 4.97E-04 & $2.16 \mathrm{E}-03$ \\
\hline & 140 & 64.952 & $2.29 \mathrm{E}+07$ & $1.92 \mathrm{E}+07$ & $1.55 \mathrm{E}-05$ & $1.29 \mathrm{E}-05$ & $6.06 \mathrm{E}-05$ \\
\hline & 147 & 64.050 & $2.75 \mathrm{E}+08$ & $2.52 \mathrm{E}+08$ & $1.78 \mathrm{E}-04$ & $1.63 \mathrm{E}-04$ & 7.75E-04 \\
\hline & 151 & 63.828 & $1.48 \mathrm{E}+08$ & $1.64 \mathrm{E}+08$ & $9.50 \mathrm{E}-05$ & $1.05 \mathrm{E}-04$ & $5.02 \mathrm{E}-04$ \\
\hline & 154 & 63.783 & $3.38 \mathrm{E}+08$ & $3.16 \mathrm{E}+08$ & $5.78 \mathrm{E}-04$ & $5.40 \mathrm{E}-04$ & $1.21 \mathrm{E}-04$ \\
\hline & 171 & 16.868 & $1.29 \mathrm{E}+10$ & $1.52 \mathrm{E}+10$ & $1.53 \mathrm{E}-04$ & $1.80 \mathrm{E}-04$ & $3.25 \mathrm{E}-03$ \\
\hline \multirow[t]{9}{*}{62} & 63 & 2324.900 & $2.02 \mathrm{E}+07$ & $1.71 \mathrm{E}+07$ & $4.91 \mathrm{E}-02$ & $4.16 \mathrm{E}-02$ & $3.76 \mathrm{E}-01$ \\
\hline & 65 & 1399.240 & $1.46 \mathrm{E}+08$ & $1.60 \mathrm{E}+08$ & $1.29 \mathrm{E}-01$ & $1.41 \mathrm{E}-01$ & $5.94 \mathrm{E}-01$ \\
\hline & 76 & 554.033 & $6.91 \mathrm{E}+06$ & $1.55 \mathrm{E}+07$ & $9.54 \mathrm{E}-04$ & $2.14 \mathrm{E}-03$ & $1.74 \mathrm{E}-03$ \\
\hline & 79 & 519.545 & $1.41 \mathrm{E}+05$ & $1.15 \mathrm{E}+06$ & $1.72 \mathrm{E}-05$ & $1.40 \mathrm{E}-04$ & $2.93 \mathrm{E}-05$ \\
\hline & 93 & 380.184 & $7.95 \mathrm{E}+06$ & $9.61 \mathrm{E}+06$ & $5.17 \mathrm{E}-04$ & $6.24 \mathrm{E}-04$ & $6.47 \mathrm{E}-04$ \\
\hline & 106 & 97.443 & $5.65 \mathrm{E}+07$ & $4.73 \mathrm{E}+07$ & $2.41 \mathrm{E}-04$ & $2.02 \mathrm{E}-04$ & $7.75 \mathrm{E}-05$ \\
\hline & 118 & 71.775 & $6.66 \mathrm{E}+10$ & $6.95 \mathrm{E}+10$ & $1.54 \mathrm{E}-01$ & $1.61 \mathrm{E}-01$ & $3.64 \mathrm{E}-02$ \\
\hline & 121 & 71.062 & $1.24 \mathrm{E}+11$ & $1.27 \mathrm{E}+11$ & $2.81 \mathrm{E}-01$ & $2.89 \mathrm{E}-01$ & $6.57 \mathrm{E}-02$ \\
\hline & 154 & 63.840 & $5.99 \mathrm{E}+08$ & $5.58 \mathrm{E}+08$ & $1.10 \mathrm{E}-03$ & $1.02 \mathrm{E}-03$ & $2.31 \mathrm{E}-04$ \\
\hline \multirow[t]{7}{*}{63} & 66 & 1975.128 & $7.04 \mathrm{E}+07$ & $5.29 \mathrm{E}+07$ & $2.06 \mathrm{E}-01$ & $1.55 \mathrm{E}-01$ & $1.34 \mathrm{E}+00$ \\
\hline & 67 & 1488.989 & $1.03 \mathrm{E}+05$ & $9.90 \mathrm{E}+04$ & $1.71 \mathrm{E}-04$ & $1.65 \mathrm{E}-04$ & 8.38E-04 \\
\hline & 71 & 1209.902 & $2.59 \mathrm{E}+05$ & $2.67 \mathrm{E}+05$ & $2.84 \mathrm{E}-04$ & $2.93 \mathrm{E}-04$ & $1.13 \mathrm{E}-03$ \\
\hline & 110 & 100.396 & $6.49 \mathrm{E}+08$ & 7.49E+08 & $4.90 \mathrm{E}-03$ & $5.66 \mathrm{E}-03$ & $1.62 \mathrm{E}-03$ \\
\hline & 122 & 72.714 & $3.44 \mathrm{E}+11$ & $3.39 \mathrm{E}+11$ & $1.36 \mathrm{E}+00$ & $1.35 \mathrm{E}+00$ & $3.27 \mathrm{E}-01$ \\
\hline & 124 & 72.231 & $2.62 \mathrm{E}+06$ & $2.63 \mathrm{E}+06$ & $1.03 \mathrm{E}-05$ & $1.03 \mathrm{E}-05$ & $2.44 \mathrm{E}-06$ \\
\hline & 141 & 66.454 & $1.37 \mathrm{E}+08$ & $1.55 \mathrm{E}+08$ & 4.55E-04 & $5.12 \mathrm{E}-04$ & $9.96 \mathrm{E}-05$ \\
\hline
\end{tabular}


Table 6. Cont.

\begin{tabular}{|c|c|c|c|c|c|c|c|}
\hline LL. & UL. & $\lambda$ & $A_{L}$ & $A_{V}$ & $f_{L}$ & $f_{V}$ & $S_{L}$ \\
\hline & 153 & 65.658 & $1.27 \mathrm{E}+07$ & $1.39 \mathrm{E}+07$ & 4.10E-05 & 4.49E-05 & 8.86E-06 \\
\hline & 169 & 17.282 & $7.27 \mathrm{E}+06$ & $1.37 \mathrm{E}+07$ & $1.63 \mathrm{E}-06$ & 3.07E-06 & $9.25 \mathrm{E}-08$ \\
\hline \multirow[t]{15}{*}{64} & 110 & 103.210 & $8.41 \mathrm{E}+07$ & $9.78 \mathrm{E}+07$ & $6.71 \mathrm{E}-04$ & $7.81 \mathrm{E}-04$ & $2.28 \mathrm{E}-04$ \\
\hline & 111 & 102.593 & $3.52 \mathrm{E}+08$ & $4.17 \mathrm{E}+08$ & $3.89 \mathrm{E}-03$ & $4.61 \mathrm{E}-03$ & $1.31 \mathrm{E}-03$ \\
\hline & 112 & 102.187 & $8.22 \mathrm{E}+08$ & $7.69 \mathrm{E}+08$ & $9.01 \mathrm{E}-03$ & $8.43 \mathrm{E}-03$ & $3.03 \mathrm{E}-03$ \\
\hline & 116 & 76.993 & $1.29 \mathrm{E}+11$ & $1.51 \mathrm{E}+11$ & $8.75 \mathrm{E}-02$ & $1.02 \mathrm{E}-01$ & $4.03 \mathrm{E}-01$ \\
\hline & 122 & 74.179 & $6.33 E+10$ & $6.14 \mathrm{E}+10$ & 2.61E-01 & $2.53 \mathrm{E}-01$ & $6.37 \mathrm{E}-02$ \\
\hline & 124 & 73.676 & $3.99 \mathrm{E}+10$ & $3.93 \mathrm{E}+10$ & $1.63 \mathrm{E}-01$ & $1.60 \mathrm{E}-01$ & 3.94E-02 \\
\hline & 125 & 73.538 & $8.71 \mathrm{E}+09$ & $8.86 \mathrm{E}+09$ & $5.13 \mathrm{E}-03$ & $5.21 \mathrm{E}-03$ & $2.15 \mathrm{E}-02$ \\
\hline & 129 & 73.286 & $4.27 \mathrm{E}+11$ & $4.24 \mathrm{E}+11$ & $2.41 \mathrm{E}+00$ & $2.39 \mathrm{E}+00$ & $5.81 \mathrm{E}-01$ \\
\hline & 131 & 72.933 & $1.69 \mathrm{E}+09$ & $1.68 \mathrm{E}+09$ & $9.44 \mathrm{E}-03$ & $9.36 \mathrm{E}-03$ & $2.27 \mathrm{E}-03$ \\
\hline & 134 & 70.147 & $5.32 \mathrm{E}+08$ & $6.99 \mathrm{E}+08$ & $1.96 \mathrm{E}-03$ & $2.58 \mathrm{E}-03$ & $4.53 \mathrm{E}-04$ \\
\hline & 141 & 67.675 & $2.45 \mathrm{E}+09$ & $2.76 \mathrm{E}+09$ & $8.41 \mathrm{E}-03$ & $9.48 \mathrm{E}-03$ & $1.87 \mathrm{E}-03$ \\
\hline & 146 & 67.245 & $5.71 E+08$ & $5.60 \mathrm{E}+08$ & $2.71 \mathrm{E}-03$ & $2.66 \mathrm{E}-03$ & $6.00 \mathrm{E}-04$ \\
\hline & 157 & 66.728 & $1.54 \mathrm{E}+07$ & $1.54 \mathrm{E}+07$ & $7.20 \mathrm{E}-05$ & $7.20 \mathrm{E}-05$ & $1.58 \mathrm{E}-05$ \\
\hline & 159 & 66.623 & $6.83 \mathrm{E}+08$ & $5.84 \mathrm{E}+08$ & $3.18 \mathrm{E}-03$ & $2.72 \mathrm{E}-03$ & $6.97 \mathrm{E}-04$ \\
\hline & 161 & 66.587 & $2.87 \mathrm{E}+07$ & $3.35 \mathrm{E}+07$ & $1.33 \mathrm{E}-04$ & $1.56 \mathrm{E}-04$ & $2.92 \mathrm{E}-05$ \\
\hline \multirow[t]{16}{*}{65} & 84 & 591.986 & $7.98 \mathrm{E}+06$ & $9.23 \mathrm{E}+06$ & $1.46 \mathrm{E}-03$ & $1.69 \mathrm{E}-03$ & $2.84 \mathrm{E}-03$ \\
\hline & 99 & 107.719 & $3.79 \mathrm{E}+07$ & $3.48 \mathrm{E}+07$ & $1.71 \mathrm{E}-04$ & $1.57 \mathrm{E}-04$ & $5.88 \mathrm{E}-05$ \\
\hline & 100 & 107.077 & $2.69 E+06$ & $3.36 \mathrm{E}+07$ & $4.36 \mathrm{E}-06$ & $5.44 \mathrm{E}-05$ & $1.49 \mathrm{E}-06$ \\
\hline & 102 & 106.566 & $9.30 \mathrm{E}+06$ & $9.95 E+06$ & $5.08 \mathrm{E}-05$ & $5.44 \mathrm{E}-05$ & $1.78 \mathrm{E}-05$ \\
\hline & 110 & 103.348 & $8.21 \mathrm{E}+07$ & $9.67 \mathrm{E}+07$ & $6.57 \mathrm{E}-04$ & 7.74E-04 & $2.24 \mathrm{E}-04$ \\
\hline & 116 & 77.071 & $4.34 \mathrm{E}+10$ & $4.71 \mathrm{E}+10$ & $1.21 \mathrm{E}-01$ & $1.31 \mathrm{E}-01$ & $2.99 \mathrm{E}-02$ \\
\hline & 117 & 76.737 & $7.81 E+10$ & $8.25 \mathrm{E}+10$ & $6.61 \mathrm{E}-02$ & $6.98 \mathrm{E}-02$ & $1.63 \mathrm{E}-02$ \\
\hline & 122 & 74.250 & $7.53 \mathrm{E}+09$ & $7.35 \mathrm{E}+09$ & $3.11 \mathrm{E}-02$ & $3.04 \mathrm{E}-02$ & $7.61 \mathrm{E}-03$ \\
\hline & 124 & 73.747 & $3.74 \mathrm{E}+11$ & $3.70 \mathrm{E}+11$ & $1.52 \mathrm{E}+00$ & $1.51 \mathrm{E}+00$ & 3.70E-01 \\
\hline & 125 & 73.612 & $1.15 \mathrm{E}+11$ & $1.14 \mathrm{E}+11$ & $2.68 \mathrm{E}-01$ & $2.66 \mathrm{E}-01$ & $6.35 \mathrm{E}-02$ \\
\hline & 134 & 70.210 & $9.54 \mathrm{E}+08$ & $1.12 \mathrm{E}+09$ & $3.52 \mathrm{E}-03$ & $4.14 \mathrm{E}-03$ & $8.15 \mathrm{E}-04$ \\
\hline & 141 & 67.735 & $6.79 \mathrm{E}+08$ & $6.19 \mathrm{E}+08$ & $2.33 \mathrm{E}-03$ & $2.13 \mathrm{E}-03$ & $5.20 \mathrm{E}-04$ \\
\hline & 143 & 67.455 & $5.19 \mathrm{E}+08$ & $5.41 \mathrm{E}+08$ & $1.11 \mathrm{E}-03$ & $1.16 \mathrm{E}-03$ & $2.46 \mathrm{E}-04$ \\
\hline & 152 & 66.924 & $8.63 \mathrm{E}+08$ & $7.11 \mathrm{E}+08$ & $1.38 \mathrm{E}-03$ & $1.14 \mathrm{E}-03$ & $2.98 \mathrm{E}-04$ \\
\hline & 169 & 66.908 & $2.39 \mathrm{E}+06$ & $2.64 \mathrm{E}+06$ & 8.02E-06 & $8.85 \mathrm{E}-06$ & $1.77 \mathrm{E}-06$ \\
\hline & 180 & 16.754 & $4.06 \mathrm{E}+08$ & $3.44 \mathrm{E}+08$ & $8.53 \mathrm{E}-05$ & $7.23 \mathrm{E}-05$ & $4.71 \mathrm{E}-06$ \\
\hline \multirow[t]{5}{*}{66} & 118 & 76.947 & $6.51 \mathrm{E}+10$ & $5.16 \mathrm{E}+10$ & $1.73 \mathrm{E}-01$ & $1.37 \mathrm{E}-01$ & 4.39E-02 \\
\hline & 120 & 76.242 & $9.00 \mathrm{E}+08$ & $7.16 \mathrm{E}+08$ & $2.35 \mathrm{E}-03$ & $1.87 \mathrm{E}-03$ & $5.88 \mathrm{E}-04$ \\
\hline & 127 & 74.666 & $7.10 \mathrm{E}+10$ & $7.04 \mathrm{E}+10$ & $2.97 \mathrm{E}-01$ & $2.94 \mathrm{E}-01$ & 7.30E-02 \\
\hline & 137 & 69.848 & $4.47 \mathrm{E}+08$ & $3.68 \mathrm{E}+08$ & $1.63 \mathrm{E}-03$ & $1.35 \mathrm{E}-03$ & $3.76 \mathrm{E}-04$ \\
\hline & 138 & 69.533 & $2.78 \mathrm{E}+08$ & $3.35 \mathrm{E}+08$ & $6.05 \mathrm{E}-04$ & 7.29E-04 & $1.39 \mathrm{E}-04$ \\
\hline
\end{tabular}


Table 6. Cont.

\begin{tabular}{|c|c|c|c|c|c|c|c|}
\hline LL. & UL. & $\lambda$ & $A_{L}$ & $A_{V}$ & $f_{L}$ & $f_{V}$ & $S_{L}$ \\
\hline \multirow[t]{7}{*}{67} & 76 & 1422.019 & $1.46 \mathrm{E}+07$ & $1.59 \mathrm{E}+07$ & $1.33 \mathrm{E}-02$ & $1.45 \mathrm{E}-02$ & $6.21 \mathrm{E}-02$ \\
\hline & 80 & 988.463 & $2.42 \mathrm{E}+07$ & $1.99 \mathrm{E}+07$ & $1.77 \mathrm{E}-02$ & $1.45 \mathrm{E}-02$ & $5.76 \mathrm{E}-02$ \\
\hline & 118 & 77.938 & $4.34 \mathrm{E}+07$ & $3.38 \mathrm{E}+07$ & 1.19E-04 & $9.22 \mathrm{E}-05$ & $3.04 \mathrm{E}-05$ \\
\hline & 120 & 77.213 & 2.42E-09 & $1.23 \mathrm{E}+09$ & $2.16 \mathrm{E}-03$ & $1.55 \mathrm{E}-03$ & $2.75 \mathrm{E}-03$ \\
\hline & 127 & 75.599 & $3.26 \mathrm{E}+10$ & $3.23 \mathrm{E}+10$ & $1.40 \mathrm{E}-01$ & $1.39 \mathrm{E}-01$ & $3.48 \mathrm{E}-02$ \\
\hline & 137 & 70.650 & $1.07 \mathrm{E}+10$ & $9.89 \mathrm{E}+09$ & 2.39E-02 & 2.22E-02 & $5.57 \mathrm{E}-03$ \\
\hline & 138 & 70.341 & $2.97 \mathrm{E}+10$ & $2.99 \mathrm{E}+10$ & $6.60 \mathrm{E}-02$ & $6.65 \mathrm{E}-02$ & $1.53 \mathrm{E}-02$ \\
\hline \multirow[t]{5}{*}{68} & 94 & 691.769 & $3.32 \mathrm{E}+05$ & $2.88 \mathrm{E}+05$ & 1.19E-04 & $1.03 \mathrm{E}-04$ & $2.71 \mathrm{E}-04$ \\
\hline & 113 & 107.543 & $1.83 \mathrm{E}+07$ & $1.98 \mathrm{E}+07$ & 2.23E-04 & $2.40 \mathrm{E}-04$ & $7.88 \mathrm{E}-05$ \\
\hline & 128 & 76.033 & $4.40 \mathrm{E}+10$ & $4.41 \mathrm{E}+10$ & 2.67E-01 & 2.67E-01 & $6.68 \mathrm{E}-02$ \\
\hline & 130 & 75.901 & $3.63 \mathrm{E}+10$ & $3.61 \mathrm{E}+10$ & 2.19E-01 & $2.18 \mathrm{E}-01$ & $5.48 \mathrm{E}-02$ \\
\hline & 150 & 69.178 & $2.76 \mathrm{E}+09$ & $2.87 \mathrm{E}+09$ & 1.39E-02 & 1.44E-02 & $3.15 \mathrm{E}-03$ \\
\hline \multirow[t]{11}{*}{69} & 76 & 1639.060 & $3.11 \mathrm{E}+06$ & $3.88 \mathrm{E}+06$ & $4.68 \mathrm{E}-03$ & $5.84 \mathrm{E}-03$ & $2.53 \mathrm{E}-02$ \\
\hline & 79 & 696.640 & $5.55 \mathrm{E}+05$ & $5.20 \mathrm{E}+05$ & 1.13E-04 & $1.06 \mathrm{E}-04$ & $2.60 \mathrm{E}-04$ \\
\hline & 94 & 692.438 & $4.57 \mathrm{E}+06$ & $5.29 \mathrm{E}+06$ & $3.75 \mathrm{E}-03$ & 4.33E-03 & $1.90 \mathrm{E}-03$ \\
\hline & 120 & 77.773 & $3.29 \mathrm{E}+08$ & $4.31 \mathrm{E}+08$ & 3.82E-04 & $5.00 \mathrm{E}-04$ & $1.95 \mathrm{E}-03$ \\
\hline & 127 & 76.135 & $6.02 \mathrm{E}+11$ & $6.10 \mathrm{E}+11$ & $6.55 \mathrm{E}-01$ & $6.65 \mathrm{E}-01$ & $2.65 \mathrm{E}+00$ \\
\hline & 137 & 71.118 & $1.40 \mathrm{E}+09$ & $1.30 \mathrm{E}+09$ & $2.95 \mathrm{E}-03$ & $2.74 \mathrm{E}-03$ & $6.92 \mathrm{E}-04$ \\
\hline & 138 & 70.805 & $3.76 \mathrm{E}+08$ & $3.21 \mathrm{E}+08$ & 7.24E-04 & $6.18 \mathrm{E}-04$ & 1.69E-04 \\
\hline & 147 & 69.425 & $5.52 \mathrm{E}+08$ & $6.20 \mathrm{E}+08$ & $4.56 \mathrm{E}-04$ & $5.12 \mathrm{E}-04$ & $2.24 \mathrm{E}-03$ \\
\hline & 151 & 69.164 & $3.20 \mathrm{E}+09$ & $3.26 \mathrm{E}+09$ & $2.61 \mathrm{E}-03$ & $2.66 \mathrm{E}-03$ & $1.17 \mathrm{E}-02$ \\
\hline & 154 & 69.111 & $2.02 \mathrm{E}+08$ & $2.19 \mathrm{E}+08$ & 4.71E-04 & $5.11 \mathrm{E}-04$ & $1.07 \mathrm{E}-04$ \\
\hline & 171 & 17.219 & $2.70 \mathrm{E}+09$ & $2.99 \mathrm{E}+09$ & $3.40 \mathrm{E}-05$ & $3.77 \mathrm{E}-05$ & $6.65 \mathrm{E}-04$ \\
\hline \multirow[t]{9}{*}{71} & 80 & 1167.194 & $9.48 \mathrm{E}+07$ & $7.59 \mathrm{E}+07$ & $9.68 \mathrm{E}-02$ & $7.75 \mathrm{E}-02$ & $3.72 \mathrm{E}-01$ \\
\hline & 103 & 111.337 & $5.59 \mathrm{E}+07$ & $4.97 \mathrm{E}+07$ & 5.19E-04 & $4.62 \mathrm{E}-04$ & $1.90 \mathrm{E}-04$ \\
\hline & 118 & 78.890 & $2.38 \mathrm{E}+07$ & $1.79 \mathrm{E}+07$ & $6.65 \mathrm{E}-05$ & 5.01E-05 & $1.73 \mathrm{E}-05$ \\
\hline & 120 & 78.150 & $1.16 \mathrm{E}+10$ & $1.14 \mathrm{E}+10$ & 5.07E-02 & $5.00 \mathrm{E}-02$ & $1.28 \mathrm{E}-02$ \\
\hline & 136 & 71.446 & $1.19 \mathrm{E}+11$ & $1.12 \mathrm{E}+11$ & $4.56 \mathrm{E}-01$ & $4.30 \mathrm{E}-01$ & $1.07 \mathrm{E}-01$ \\
\hline & 137 & 71.432 & $4.37 \mathrm{E}+10$ & $4.29 \mathrm{E}+10$ & $1.00 \mathrm{E}-01$ & 9.83E-02 & $2.36 \mathrm{E}-02$ \\
\hline & 138 & 71.116 & $1.10 \mathrm{E}+11$ & $1.04 \mathrm{E}+11$ & 2.49E-01 & $2.37 \mathrm{E}-01$ & $5.84 \mathrm{E}-02$ \\
\hline & 154 & 69.408 & $1.58 \mathrm{E}+08$ & $1.53 \mathrm{E}+08$ & 3.42E-04 & 3.31E-04 & $7.81 \mathrm{E}-05$ \\
\hline & 176 & 17.058 & $2.17 \mathrm{E}+10$ & $2.09 \mathrm{E}+10$ & 2.84E-03 & $2.74 \mathrm{E}-03$ & $1.59 \mathrm{E}-04$ \\
\hline \multirow[t]{6}{*}{72} & 99 & 114.397 & $1.93 \mathrm{E}+07$ & $1.84 \mathrm{E}+07$ & 4.27E-05 & 4.09E-05 & $1.09 \mathrm{E}-04$ \\
\hline & 110 & 109.486 & $9.32 \mathrm{E}+06$ & $9.54 \mathrm{E}+06$ & 8.37E-05 & 8.57E-05 & $3.02 \mathrm{E}-05$ \\
\hline & 129 & 76.395 & $1.93 \mathrm{E}+09$ & $1.99 \mathrm{E}+09$ & $1.18 \mathrm{E}-02$ & $1.22 \mathrm{E}-02$ & $2.97 \mathrm{E}-03$ \\
\hline & 131 & 76.012 & $1.11 \mathrm{E}+12$ & $1.11 \mathrm{E}+12$ & $6.75 \mathrm{E}+00$ & $6.71 \mathrm{E}+00$ & $1.69 \mathrm{E}+00$ \\
\hline & 134 & 72.990 & $2.10 \mathrm{E}+07$ & $1.71 \mathrm{E}+07$ & 8.38E-05 & $6.83 \mathrm{E}-05$ & $2.01 \mathrm{E}-05$ \\
\hline & 135 & 72.827 & $1.25 \mathrm{E}+08$ & $1.14 \mathrm{E}+08$ & 7.13E-05 & $6.54 \mathrm{E}-05$ & $2.73 \mathrm{E}-04$ \\
\hline
\end{tabular}


Table 6. Cont.

\begin{tabular}{|c|c|c|c|c|c|c|c|}
\hline LL. & UL. & $\lambda$ & $A_{L}$ & $A_{V}$ & $f_{L}$ & $f_{V}$ & $S_{L}$ \\
\hline & 141 & 70.318 & $2.14 \mathrm{E}+08$ & $2.58 \mathrm{E}+08$ & 7.94E-04 & $9.56 \mathrm{E}-04$ & $1.84 \mathrm{E}-04$ \\
\hline & 146 & 69.854 & $8.01 \mathrm{E}+07$ & $7.48 \mathrm{E}+07$ & $4.10 \mathrm{E}-04$ & $3.83 \mathrm{E}-04$ & $9.43 \mathrm{E}-05$ \\
\hline & 157 & 69.297 & $2.43 \mathrm{E}+09$ & $2.29 \mathrm{E}+09$ & $1.22 \mathrm{E}-02$ & $1.15 \mathrm{E}-02$ & $2.79 \mathrm{E}-03$ \\
\hline & 159 & 69.183 & $6.45 \mathrm{E}+08$ & $6.80 \mathrm{E}+08$ & $3.24 \mathrm{E}-03$ & $3.41 \mathrm{E}-03$ & 7.38E-04 \\
\hline & 161 & 69.144 & $1.50 \mathrm{E}+08$ & $1.41 \mathrm{E}+08$ & 7.53E-04 & 7.07E-04 & $1.71 \mathrm{E}-04$ \\
\hline \multirow[t]{12}{*}{73} & 81 & 1002.770 & $5.73 \mathrm{E}+05$ & $5.91 \mathrm{E}+05$ & $4.32 \mathrm{E}-04$ & 4.45E-04 & $1.43 \mathrm{E}-03$ \\
\hline & 83 & 883.312 & $4.08 \mathrm{E}+05$ & $4.03 \mathrm{E}+05$ & $3.34 \mathrm{E}-04$ & $3.30 \mathrm{E}-04$ & $9.72 \mathrm{E}-04$ \\
\hline & 85 & 862.507 & $5.44 \mathrm{E}+05$ & $5.77 \mathrm{E}+05$ & $3.03 \mathrm{E}-04$ & $3.22 \mathrm{E}-04$ & 8.62E-04 \\
\hline & 95 & 712.144 & $1.82 \mathrm{E}+06$ & $1.72 \mathrm{E}+06$ & $9.55 \mathrm{E}-04$ & $9.01 \mathrm{E}-04$ & $2.22 \mathrm{E}-03$ \\
\hline & 101 & 113.362 & $6.33 E+04$ & $2.10 \mathrm{E}+05$ & $6.10 \mathrm{E}-07$ & 2.02E-06 & $2.28 \mathrm{E}-07$ \\
\hline & 110 & 109.496 & $2.43 \mathrm{E}+08$ & $5.04 \mathrm{E}+07$ & 2.19E-03 & 4.53E-04 & $7.88 \mathrm{E}-04$ \\
\hline & 131 & 76.016 & $5.51 \mathrm{E}+10$ & $5.45 \mathrm{E}+10$ & $3.34 \mathrm{E}-01$ & $3.31 \mathrm{E}-01$ & $8.36 \mathrm{E}-02$ \\
\hline & 134 & 72.994 & $9.87 \mathrm{E}+05$ & $8.45 \mathrm{E}+05$ & $3.94 \mathrm{E}-06$ & $3.37 \mathrm{E}-06$ & $9.47 \mathrm{E}-07$ \\
\hline & 146 & 69.858 & $6.20 \mathrm{E}+05$ & $6.17 \mathrm{E}+05$ & $3.17 \mathrm{E}-06$ & $3.16 \mathrm{E}-06$ & 7.30E-07 \\
\hline & 157 & 69.300 & $1.37 \mathrm{E}+08$ & $1.26 \mathrm{E}+08$ & $6.89 \mathrm{E}-04$ & $6.35 \mathrm{E}-04$ & $1.57 \mathrm{E}-04$ \\
\hline & 159 & 69.186 & $7.17 \mathrm{E}+06$ & $8.35 \mathrm{E}+06$ & $3.60 \mathrm{E}-05$ & 4.19E-05 & $8.20 \mathrm{E}-06$ \\
\hline & 161 & 69.148 & $2.32 \mathrm{E}+07$ & $2.92 \mathrm{E}+07$ & $1.16 \mathrm{E}-04$ & $1.46 \mathrm{E}-04$ & $2.65 \mathrm{E}-05$ \\
\hline \multirow[t]{6}{*}{74} & 95 & 734.939 & $2.28 \mathrm{E}+06$ & $2.05 \mathrm{E}+06$ & $1.27 \mathrm{E}-03$ & $1.14 \mathrm{E}-03$ & $3.05 \mathrm{E}-03$ \\
\hline & 131 & 76.270 & $4.27 \mathrm{E}+10$ & $4.26 \mathrm{E}+10$ & $2.61 \mathrm{E}-01$ & $2.60 \mathrm{E}-01$ & $6.55 \mathrm{E}-02$ \\
\hline & 153 & 69.644 & $5.28 \mathrm{E}+07$ & $5.88 \mathrm{E}+07$ & $1.92 \mathrm{E}-04$ & $2.14 \mathrm{E}-04$ & $4.40 \mathrm{E}-05$ \\
\hline & 157 & 69.511 & $1.27 \mathrm{E}+08$ & $1.26 \mathrm{E}+08$ & $6.44 \mathrm{E}-04$ & $6.37 \mathrm{E}-04$ & $1.47 \mathrm{E}-04$ \\
\hline & 159 & 69.397 & $7.74 \mathrm{E}+07$ & $8.48 \mathrm{E}+07$ & $3.91 \mathrm{E}-04$ & 4.29E-04 & 8.93E-05 \\
\hline & 161 & 69.358 & $6.27 \mathrm{E}+08$ & $6.05 \mathrm{E}+08$ & $3.17 \mathrm{E}-03$ & $3.05 \mathrm{E}-03$ & $7.23 \mathrm{E}-04$ \\
\hline \multirow[t]{12}{*}{75} & 76 & 2035.960 & $5.14 \mathrm{E}+07$ & $5.40 \mathrm{E}+07$ & $1.01 \mathrm{E}-01$ & $1.06 \mathrm{E}-01$ & $6.75 \mathrm{E}-01$ \\
\hline & 79 & 1636.710 & $2.66 \mathrm{E}+07$ & $2.67 \mathrm{E}+07$ & $3.21 \mathrm{E}-02$ & $3.22 \mathrm{E}-02$ & $1.73 \mathrm{E}-01$ \\
\hline & 105 & 110.667 & $1.80 \mathrm{E}+09$ & $1.97 \mathrm{E}+09$ & $6.03 \mathrm{E}-03$ & $6.58 \mathrm{E}-03$ & $1.81 \mathrm{E}-02$ \\
\hline & 127 & 76.830 & $1.60 \mathrm{E}+09$ & $1.65 \mathrm{E}+09$ & $1.79 \mathrm{E}-03$ & $1.84 \mathrm{E}-03$ & $7.28 \mathrm{E}-03$ \\
\hline & 136 & 71.739 & $5.07 \mathrm{E}+10$ & $5.22 \mathrm{E}+10$ & $4.62 \mathrm{E}-02$ & $4.76 \mathrm{E}-02$ & $2.02 \mathrm{E}-01$ \\
\hline & 137 & 71.724 & $1.37 \mathrm{E}+11$ & $1.48 \mathrm{E}+11$ & $3.42 \mathrm{E}-01$ & $3.69 \mathrm{E}-01$ & 8.07E-02 \\
\hline & 138 & 71.406 & $4.30 \mathrm{E}+10$ & $4.70 \mathrm{E}+10$ & $1.08 \mathrm{E}-01$ & $1.18 \mathrm{E}-01$ & $2.53 \mathrm{E}-02$ \\
\hline & 140 & 71.081 & $1.45 \mathrm{E}+11$ & $1.49 \mathrm{E}+11$ & $1.28 \mathrm{E}-01$ & $1.32 \mathrm{E}-01$ & $5.65 \mathrm{E}-01$ \\
\hline & 147 & 70.003 & $7.84 \mathrm{E}+08$ & $8.06 \mathrm{E}+08$ & $6.64 \mathrm{E}-04$ & $6.82 \mathrm{E}-04$ & $2.96 \mathrm{E}-03$ \\
\hline & 151 & 69.738 & $5.08 \mathrm{E}+08$ & $4.18 \mathrm{E}+08$ & $4.25 \mathrm{E}-04$ & $3.50 \mathrm{E}-04$ & $1.52 \mathrm{E}-03$ \\
\hline & 154 & 69.684 & $6.84 \mathrm{E}+08$ & $6.79 \mathrm{E}+08$ & $1.48 \mathrm{E}-03$ & $1.47 \mathrm{E}-03$ & $3.40 \mathrm{E}-04$ \\
\hline & 171 & 17.254 & $1.27 \mathrm{E}+10$ & $1.56 \mathrm{E}+10$ & $1.61 \mathrm{E}-04$ & $1.98 \mathrm{E}-04$ & $3.49 \mathrm{E}-03$ \\
\hline \multirow[t]{3}{*}{76} & 125 & 80.031 & $3.03 \mathrm{E}+09$ & $3.44 \mathrm{E}+09$ & $9.90 \mathrm{E}-03$ & $1.12 \mathrm{E}-02$ & $2.61 \mathrm{E}-03$ \\
\hline & 134 & 75.854 & $5.44 \mathrm{E}+10$ & $5.92 \mathrm{E}+10$ & $1.53 \mathrm{E}-01$ & $1.67 \mathrm{E}-01$ & $3.83 \mathrm{E}-02$ \\
\hline & 143 & 72.809 & $8.37 \mathrm{E}+10$ & $8.53 \mathrm{E}+10$ & 2.03E-01 & 2.07E-01 & 4.88E-02 \\
\hline
\end{tabular}


Table 6. Cont.

\begin{tabular}{|c|c|c|c|c|c|c|c|}
\hline LL. & UL. & $\lambda$ & $A_{L}$ & $A_{V}$ & $f_{L}$ & $f_{V}$ & $S_{L}$ \\
\hline & 149 & 72.327 & $9.60 \mathrm{E}+10$ & $9.31 \mathrm{E}+10$ & $7.53 \mathrm{E}-02$ & $7.30 \mathrm{E}-02$ & $1.79 \mathrm{E}-02$ \\
\hline & 152 & 72.190 & $1.13 \mathrm{E}+11$ & $1.11 \mathrm{E}+11$ & 2.60E-01 & $2.55 \mathrm{E}-01$ & $6.18 \mathrm{E}-02$ \\
\hline & 167 & 17.793 & $4.12 \mathrm{E}+06$ & $4.65 \mathrm{E}+08$ & $1.96 \mathrm{E}-07$ & $2.21 \mathrm{E}-05$ & $1.15 \mathrm{E}-08$ \\
\hline & 168 & 17.788 & $9.75 \mathrm{E}+08$ & $9.26 \mathrm{E}+08$ & $1.32 \mathrm{E}-04$ & $1.25 \mathrm{E}-04$ & 7.72E-06 \\
\hline \multirow[t]{16}{*}{77} & 110 & 117.075 & $2.47 \mathrm{E}+07$ & $2.96 \mathrm{E}+07$ & $2.54 \mathrm{E}-04$ & $3.04 \mathrm{E}-04$ & $9.79 \mathrm{E}-05$ \\
\hline & 111 & 116.282 & $2.13 \mathrm{E}+08$ & $2.43 \mathrm{E}+08$ & $3.02 \mathrm{E}-03$ & $3.45 \mathrm{E}-03$ & $1.16 \mathrm{E}-03$ \\
\hline & 112 & 115.760 & $3.17 \mathrm{E}+08$ & $2.75 E+08$ & $4.46 \mathrm{E}-03$ & $3.87 \mathrm{E}-03$ & $1.70 \mathrm{E}-03$ \\
\hline & 122 & 81.080 & $9.20 \mathrm{E}+08$ & $7.99 \mathrm{E}+08$ & $4.53 \mathrm{E}-03$ & $3.94 \mathrm{E}-03$ & $1.21 \mathrm{E}-03$ \\
\hline & 129 & 80.014 & $1.83 \mathrm{E}+09$ & $1.88 \mathrm{E}+09$ & $1.23 \mathrm{E}-02$ & $1.26 \mathrm{E}-02$ & $3.24 \mathrm{E}-03$ \\
\hline & 131 & 79.593 & $6.80 \mathrm{E}+08$ & $6.72 \mathrm{E}+08$ & $4.52 \mathrm{E}-03$ & 4.47E-03 & $1.18 \mathrm{E}-03$ \\
\hline & 134 & 76.287 & $7.46 \mathrm{E}+10$ & $6.37 \mathrm{E}+10$ & $3.25 \mathrm{E}-01$ & $2.78 \mathrm{E}-01$ & 8.17E-02 \\
\hline & 135 & 76.109 & $2.42 \mathrm{E}+10$ & $2.98 \mathrm{E}+10$ & $1.58 \mathrm{E}-02$ & $1.95 \mathrm{E}-02$ & $7.76 \mathrm{E}-02$ \\
\hline & 141 & 73.373 & $8.72 \mathrm{E}+10$ & $8.42 \mathrm{E}+10$ & $3.52 \mathrm{E}-01$ & $3.40 \mathrm{E}-01$ & $8.50 \mathrm{E}-02$ \\
\hline & 143 & 73.044 & $8.31 \mathrm{E}+10$ & $8.27 \mathrm{E}+10$ & 4.79E-02 & $4.77 \mathrm{E}-02$ & $1.98 \mathrm{E}-01$ \\
\hline & 146 & 72.868 & $2.78 \mathrm{E}+11$ & $2.75 E+11$ & $1.55 \mathrm{E}+00$ & $1.53 \mathrm{E}+00$ & $3.72 \mathrm{E}-01$ \\
\hline & 152 & 72.421 & $2.87 \mathrm{E}+08$ & $1.89 \mathrm{E}+08$ & $1.62 \mathrm{E}-04$ & $1.07 \mathrm{E}-04$ & 4.47E-04 \\
\hline & 157 & 72.261 & $4.98 \mathrm{E}+08$ & $4.96 \mathrm{E}+08$ & $2.73 \mathrm{E}-03$ & $2.72 \mathrm{E}-03$ & $6.49 \mathrm{E}-04$ \\
\hline & 159 & 72.137 & $3.53 \mathrm{E}+10$ & $3.57 \mathrm{E}+10$ & $1.93 \mathrm{E}-01$ & $1.95 \mathrm{E}-01$ & $4.58 \mathrm{E}-02$ \\
\hline & 161 & 72.095 & $6.29 \mathrm{E}+09$ & $6.30 \mathrm{E}+09$ & $3.43 \mathrm{E}-02$ & $3.44 \mathrm{E}-02$ & $8.14 \mathrm{E}-03$ \\
\hline & 168 & 17.802 & $8.90 \mathrm{E}+07$ & $1.01 \mathrm{E}+08$ & 7.44E-07 & $8.41 \mathrm{E}-07$ & $1.43 \mathrm{E}-05$ \\
\hline \multirow[t]{13}{*}{78} & 110 & 118.109 & $1.96 \mathrm{E}+06$ & $2.34 \mathrm{E}+06$ & $2.05 \mathrm{E}-05$ & $2.45 \mathrm{E}-05$ & $7.96 \mathrm{E}-06$ \\
\hline & 111 & 117.302 & $4.88 \mathrm{E}+07$ & $5.87 \mathrm{E}+07$ & $7.05 \mathrm{E}-04$ & $8.47 \mathrm{E}-04$ & $2.72 \mathrm{E}-04$ \\
\hline & 112 & 116.771 & $8.25 \mathrm{E}+05$ & $8.34 \mathrm{E}+05$ & $1.18 \mathrm{E}-05$ & $1.19 \mathrm{E}-05$ & $4.54 \mathrm{E}-06$ \\
\hline & 129 & 80.496 & $9.64 \mathrm{E}+07$ & $9.12 \mathrm{E}+07$ & $6.55 \mathrm{E}-04$ & $6.20 \mathrm{E}-04$ & $1.74 \mathrm{E}-04$ \\
\hline & 131 & 80.070 & $1.77 \mathrm{E}+07$ & $1.71 \mathrm{E}+07$ & $1.19 \mathrm{E}-04$ & $1.15 \mathrm{E}-04$ & $3.13 \mathrm{E}-05$ \\
\hline & 134 & 76.724 & $1.15 \mathrm{E}+11$ & $1.01 \mathrm{E}+11$ & 5.09E-01 & 4.47E-01 & $1.29 \mathrm{E}-01$ \\
\hline & 140 & 73.778 & $2.80 \mathrm{E}+09$ & $2.62 \mathrm{E}+09$ & $1.14 \mathrm{E}-02$ & $1.07 \mathrm{E}-02$ & $2.78 \mathrm{E}-03$ \\
\hline & 144 & 73.366 & $2.83 \mathrm{E}+10$ & $2.97 \mathrm{E}+10$ & $1.14 \mathrm{E}-01$ & $1.20 \mathrm{E}-01$ & $2.76 \mathrm{E}-02$ \\
\hline & 146 & 73.267 & $1.34 \mathrm{E}+11$ & $1.33 \mathrm{E}+11$ & $7.55 \mathrm{E}-01$ & $7.50 \mathrm{E}-01$ & $1.82 \mathrm{E}-01$ \\
\hline & 157 & 72.654 & $1.46 \mathrm{E}+07$ & $1.41 \mathrm{E}+07$ & $8.08 \mathrm{E}-05$ & $7.78 \mathrm{E}-05$ & $1.93 \mathrm{E}-05$ \\
\hline & 159 & 72.528 & $6.22 \mathrm{E}+09$ & $6.17 \mathrm{E}+09$ & $3.43 \mathrm{E}-02$ & $3.41 \mathrm{E}-02$ & 8.19E-03 \\
\hline & 161 & 72.486 & $4.23 \mathrm{E}+08$ & $4.23 \mathrm{E}+08$ & $2.33 \mathrm{E}-03$ & $2.33 \mathrm{E}-03$ & $5.57 \mathrm{E}-04$ \\
\hline & 180 & 17.101 & $4.03 E+06$ & $4.80 \mathrm{E}+06$ & 8.83E-07 & $1.05 \mathrm{E}-06$ & 4.97E-08 \\
\hline \multirow[t]{6}{*}{79} & 122 & 81.580 & $3.33 \mathrm{E}+07$ & $3.71 \mathrm{E}+07$ & $1.66 \mathrm{E}-04$ & $1.85 \mathrm{E}-04$ & $4.46 \mathrm{E}-05$ \\
\hline & 124 & 80.973 & $2.79 \mathrm{E}+08$ & $2.36 \mathrm{E}+08$ & $1.37 \mathrm{E}-03$ & $1.16 \mathrm{E}-03$ & $3.65 \mathrm{E}-04$ \\
\hline & 134 & 76.549 & $1.17 \mathrm{E}+10$ & $1.27 \mathrm{E}+10$ & $3.33 \mathrm{E}-02$ & $3.61 \mathrm{E}-02$ & $8.40 \mathrm{E}-03$ \\
\hline & 141 & 73.782 & $1.83 \mathrm{E}+10$ & $1.79 \mathrm{E}+10$ & $7.48 \mathrm{E}-02$ & 7.31E-02 & $1.82 \mathrm{E}-02$ \\
\hline & 143 & 73.450 & $2.17 \mathrm{E}+11$ & $2.20 \mathrm{E}+11$ & 5.33E-01 & $5.40 \mathrm{E}-01$ & $1.29 \mathrm{E}-01$ \\
\hline & 144 & 73.370 & $1.71 \mathrm{E}+11$ & $1.69 \mathrm{E}+11$ & $6.89 \mathrm{E}-01$ & $6.83 \mathrm{E}-01$ & $1.66 \mathrm{E}-01$ \\
\hline
\end{tabular}


Table 6. Cont.

\begin{tabular}{|c|c|c|c|c|c|c|c|}
\hline LL. & UL. & $\lambda$ & $A_{L}$ & $A_{V}$ & $f_{L}$ & $f_{V}$ & $S_{L}$ \\
\hline & 149 & 72.959 & $3.39 \mathrm{E}+11$ & $3.34 \mathrm{E}+11$ & $2.71 \mathrm{E}-01$ & $2.67 \mathrm{E}-01$ & $6.50 \mathrm{E}-02$ \\
\hline & 152 & 72.820 & $6.09 E+10$ & $6.08 \mathrm{E}+10$ & $1.45 \mathrm{E}-01$ & $1.45 \mathrm{E}-01$ & $3.48 \mathrm{E}-02$ \\
\hline & 153 & 72.803 & $1.67 \mathrm{E}+06$ & $1.72 \mathrm{E}+06$ & $6.65 \mathrm{E}-06$ & $6.85 \mathrm{E}-06$ & $1.59 \mathrm{E}-06$ \\
\hline & 170 & 17.454 & $5.75 \mathrm{E}+08$ & $5.50 \mathrm{E}+08$ & $7.53 \mathrm{E}-05$ & $7.20 \mathrm{E}-05$ & 4.33E-06 \\
\hline \multirow[t]{12}{*}{80} & 101 & 125.536 & $1.06 \mathrm{E}+07$ & $1.31 \mathrm{E}+07$ & $1.25 \mathrm{E}-04$ & $1.55 \mathrm{E}-04$ & $5.17 \mathrm{E}-05$ \\
\hline & 110 & 120.813 & $8.90 \mathrm{E}+05$ & $1.07 \mathrm{E}+06$ & $9.74 \mathrm{E}-06$ & $1.17 \mathrm{E}-05$ & $3.87 \mathrm{E}-06$ \\
\hline & 111 & 119.968 & $3.77 \mathrm{E}+07$ & $4.00 \mathrm{E}+07$ & $5.70 \mathrm{E}-04$ & $6.04 \mathrm{E}-04$ & $2.25 \mathrm{E}-04$ \\
\hline & 112 & 119.413 & $8.09 \mathrm{E}+08$ & $7.07 \mathrm{E}+08$ & $1.21 \mathrm{E}-02$ & $1.06 \mathrm{E}-02$ & $4.76 \mathrm{E}-03$ \\
\hline & 131 & 81.303 & $5.15 \mathrm{E}+08$ & $5.05 \mathrm{E}+08$ & $3.57 \mathrm{E}-03$ & $3.50 \mathrm{E}-03$ & $9.56 \mathrm{E}-04$ \\
\hline & 134 & 77.856 & $2.98 \mathrm{E}+10$ & $2.51 \mathrm{E}+10$ & $1.35 \mathrm{E}-01$ & $1.14 \mathrm{E}-01$ & $3.47 \mathrm{E}-02$ \\
\hline & 135 & 77.671 & $1.02 \mathrm{E}+11$ & $1.26 \mathrm{E}+11$ & 7.07E-02 & $8.77 \mathrm{E}-02$ & $3.43 \mathrm{E}-01$ \\
\hline & 141 & 74.823 & $3.29 \mathrm{E}+10$ & $3.07 \mathrm{E}+10$ & $1.38 \mathrm{E}-01$ & $1.29 \mathrm{E}-01$ & $3.40 \mathrm{E}-02$ \\
\hline & 146 & 74.298 & $1.62 \mathrm{E}+10$ & $1.57 \mathrm{E}+10$ & $9.41 \mathrm{E}-02$ & 9.09E-02 & $2.30 \mathrm{E}-02$ \\
\hline & 157 & 73.668 & $4.69 \mathrm{E}+09$ & $4.64 \mathrm{E}+09$ & $2.67 \mathrm{E}-02$ & 2.64E-02 & $6.47 \mathrm{E}-03$ \\
\hline & 159 & 73.539 & $3.37 \mathrm{E}+11$ & $3.36 \mathrm{E}+11$ & $1.91 \mathrm{E}+00$ & $1.91 \mathrm{E}+00$ & 4.63E-01 \\
\hline & 161 & 73.496 & $4.47 \mathrm{E}+10$ & $4.45 \mathrm{E}+10$ & $2.53 \mathrm{E}-01$ & $2.52 \mathrm{E}-01$ & $6.13 \mathrm{E}-02$ \\
\hline \multirow[t]{5}{*}{81} & 120 & 84.764 & $7.78 \mathrm{E}+08$ & $6.76 \mathrm{E}+08$ & $4.00 \mathrm{E}-03$ & $3.48 \mathrm{E}-03$ & $1.09 \mathrm{E}-03$ \\
\hline & 121 & 84.625 & $4.86 \mathrm{E}+10$ & $3.72 \mathrm{E}+10$ & 1.29E-01 & $9.90 \mathrm{E}-02$ & $3.28 \mathrm{E}-02$ \\
\hline & 136 & 76.934 & $9.06 \mathrm{E}+09$ & $6.56 \mathrm{E}+09$ & 8.04E-03 & $5.82 \mathrm{E}-03$ & $1.02 \mathrm{E}-02$ \\
\hline & 137 & 76.552 & $5.34 \mathrm{E}+09$ & $4.28 \mathrm{E}+09$ & $1.41 \mathrm{E}-02$ & $1.13 \mathrm{E}-02$ & $3.55 \mathrm{E}-03$ \\
\hline & 154 & 74.576 & $5.12 \mathrm{E}+09$ & $5.30 \mathrm{E}+09$ & $1.28 \mathrm{E}-02$ & $1.33 \mathrm{E}-02$ & $3.14 \mathrm{E}-03$ \\
\hline \multirow[t]{4}{*}{82} & 99 & 131.066 & $9.36 \mathrm{E}+07$ & $9.31 \mathrm{E}+07$ & 7.23E-04 & 7.19E-04 & $3.12 \mathrm{E}-04$ \\
\hline & 134 & 79.244 & $5.12 \mathrm{E}+10$ & $3.53 \mathrm{E}+10$ & $1.45 \mathrm{E}-01$ & $9.98 \mathrm{E}-02$ & $3.77 \mathrm{E}-02$ \\
\hline & 143 & 75.927 & $3.65 \mathrm{E}+09$ & $3.47 \mathrm{E}+09$ & $9.47 \mathrm{E}-03$ & $9.01 \mathrm{E}-03$ & $2.37 \mathrm{E}-03$ \\
\hline & 152 & 75.254 & $2.31 \mathrm{E}+11$ & $2.18 \mathrm{E}+11$ & $5.89 \mathrm{E}-01$ & $5.54 \mathrm{E}-01$ & $1.46 \mathrm{E}-01$ \\
\hline \multirow[t]{6}{*}{83} & 113 & 124.258 & $5.57 \mathrm{E}+06$ & $6.19 \mathrm{E}+06$ & $8.90 \mathrm{E}-05$ & $9.90 \mathrm{E}-05$ & $3.62 \mathrm{E}-05$ \\
\hline & 128 & 84.023 & $5.54 \mathrm{E}+08$ & $5.12 \mathrm{E}+08$ & $4.06 \mathrm{E}-03$ & $3.76 \mathrm{E}-03$ & $1.12 \mathrm{E}-03$ \\
\hline & 130 & 83.864 & $4.84 \mathrm{E}+07$ & $4.90 \mathrm{E}+07$ & $3.54 \mathrm{E}-04$ & $3.58 \mathrm{E}-04$ & $9.72 \mathrm{E}-05$ \\
\hline & 150 & 75.729 & $1.06 \mathrm{E}+11$ & $1.05 \mathrm{E}+11$ & $6.33 \mathrm{E}-01$ & $6.27 \mathrm{E}-01$ & $1.57 \mathrm{E}-01$ \\
\hline & 156 & 75.536 & $1.14 \mathrm{E}+11$ & $1.12 \mathrm{E}+11$ & $6.77 \mathrm{E}-01$ & $6.68 \mathrm{E}-01$ & $1.68 \mathrm{E}-01$ \\
\hline & 174 & 17.468 & $4.41 \mathrm{E}+08$ & $4.21 \mathrm{E}+08$ & $1.41 \mathrm{E}-04$ & $1.35 \mathrm{E}-04$ & $8.10 \mathrm{E}-06$ \\
\hline \multirow[t]{5}{*}{84} & 127 & 85.014 & $9.02 \mathrm{E}+07$ & $7.43 \mathrm{E}+07$ & $1.31 \mathrm{E}-04$ & $1.08 \mathrm{E}-04$ & $3.92 \mathrm{E}-04$ \\
\hline & 136 & 78.825 & $7.77 \mathrm{E}+09$ & $1.02 \mathrm{E}+10$ & $9.04 \mathrm{E}-03$ & $1.18 \mathrm{E}-02$ & $4.62 \mathrm{E}-02$ \\
\hline & 147 & 76.732 & $3.64 \mathrm{E}+11$ & $3.68 \mathrm{E}+11$ & $3.91 \mathrm{E}-01$ & $3.95 \mathrm{E}-01$ & $1.58 \mathrm{E}+00$ \\
\hline & 151 & 76.416 & $4.23 \mathrm{E}+10$ & $4.24 \mathrm{E}+10$ & 4.49E-02 & 4.49E-02 & $1.81 \mathrm{E}-01$ \\
\hline & 154 & 76.350 & $3.38 \mathrm{E}+11$ & $3.42 \mathrm{E}+11$ & $8.75 \mathrm{E}-01$ & $8.85 \mathrm{E}-01$ & $2.17 \mathrm{E}-01$ \\
\hline \multirow[t]{2}{*}{85} & 91 & 8156.857 & $4.05 \mathrm{E}+04$ & $4.26 \mathrm{E}+04$ & $2.81 \mathrm{E}-03$ & $2.96 \mathrm{E}-03$ & 7.54E-02 \\
\hline & 118 & 86.848 & $1.32 \mathrm{E}+08$ & $1.01 \mathrm{E}+08$ & $4.36 \mathrm{E}-04$ & $3.33 \mathrm{E}-04$ & $1.23 \mathrm{E}-04$ \\
\hline
\end{tabular}


Table 6. Cont.

\begin{tabular}{|c|c|c|c|c|c|c|c|}
\hline LL. & UL. & $\lambda$ & $A_{L}$ & $A_{V}$ & $f_{L}$ & $f_{V}$ & $S_{L}$ \\
\hline & 120 & 85.945 & $3.98 \mathrm{E}+07$ & $3.31 \mathrm{E}+07$ & $2.10 \mathrm{E}-04$ & $1.75 \mathrm{E}-04$ & $5.80 \mathrm{E}-05$ \\
\hline & 138 & 77.519 & $4.35 \mathrm{E}+10$ & $4.31 \mathrm{E}+10$ & $1.11 \mathrm{E}-01$ & $1.11 \mathrm{E}-01$ & $2.77 \mathrm{E}-02$ \\
\hline \multirow[t]{3}{*}{88} & 137 & 78.261 & $4.50 \mathrm{E}+09$ & $5.50 \mathrm{E}+09$ & $1.24 \mathrm{E}-02$ & $1.51 \mathrm{E}-02$ & $3.19 \mathrm{E}-03$ \\
\hline & 138 & 77.883 & $9.34 \mathrm{E}+09$ & $1.18 \mathrm{E}+10$ & $2.55 \mathrm{E}-02$ & $3.22 \mathrm{E}-02$ & $6.53 \mathrm{E}-03$ \\
\hline & 154 & 75.839 & $3.40 \mathrm{E}+11$ & $3.39 \mathrm{E}+11$ & 8.80E-01 & 8.76E-01 & $2.20 \mathrm{E}-01$ \\
\hline \multirow[t]{5}{*}{89} & 129 & 84.601 & $4.52 \mathrm{E}+07$ & $4.00 \mathrm{E}+07$ & $3.40 \mathrm{E}-04$ & 3.01E-04 & $9.46 \mathrm{E}-05$ \\
\hline & 131 & 84.131 & $2.60 \mathrm{E}+09$ & $2.67 \mathrm{E}+09$ & $1.93 \mathrm{E}-02$ & 1.99E-02 & $5.34 \mathrm{E}-03$ \\
\hline & 157 & 75.982 & $9.62 \mathrm{E}+11$ & $9.55 \mathrm{E}+11$ & $5.83 \mathrm{E}+00$ & $5.79 \mathrm{E}+00$ & $1.46 \mathrm{E}+00$ \\
\hline & 159 & 75.845 & $1.47 \mathrm{E}+09$ & $1.40 \mathrm{E}+09$ & 8.88E-03 & 8.43E-03 & $2.22 \mathrm{E}-03$ \\
\hline & 161 & 75.799 & $5.56 \mathrm{E}+09$ & $5.51 \mathrm{E}+09$ & $3.35 \mathrm{E}-02$ & 3.32E-02 & $8.36 \mathrm{E}-03$ \\
\hline \multirow[t]{5}{*}{91} & 146 & 76.732 & $1.43 \mathrm{E}+08$ & $1.36 \mathrm{E}+08$ & $1.06 \mathrm{E}-03$ & $1.01 \mathrm{E}-03$ & $2.94 \mathrm{E}-04$ \\
\hline & 153 & 76.218 & $6.14 \mathrm{E}+09$ & $5.92 \mathrm{E}+09$ & 2.67E-02 & $2.58 \mathrm{E}-02$ & $6.70 \mathrm{E}-03$ \\
\hline & 157 & 76.059 & $1.66 \mathrm{E}+11$ & $1.65 \mathrm{E}+11$ & $1.00 \mathrm{E}+00$ & $1.00 \mathrm{E}+00$ & $2.52 \mathrm{E}-01$ \\
\hline & 161 & 75.876 & $2.76 \mathrm{E}+08$ & $3.05 \mathrm{E}+08$ & $1.67 \mathrm{E}-03$ & $1.84 \mathrm{E}-03$ & 4.17E-04 \\
\hline & 179 & 17.200 & $3.49 \mathrm{E}+07$ & $4.63 \mathrm{E}+07$ & $1.08 \mathrm{E}-05$ & $1.44 \mathrm{E}-05$ & $6.14 \mathrm{E}-07$ \\
\hline \multirow[t]{4}{*}{92} & 131 & 84.750 & $1.05 \mathrm{E}+08$ & $1.06 \mathrm{E}+08$ & 7.91E-04 & 7.97E-04 & $2.21 \mathrm{E}-04$ \\
\hline & 157 & 76.486 & $5.48 \mathrm{E}+10$ & $5.46 \mathrm{E}+10$ & 3.36E-01 & $3.35 \mathrm{E}-01$ & $8.47 \mathrm{E}-02$ \\
\hline & 159 & 76.347 & $1.75 \mathrm{E}+10$ & $1.54 \mathrm{E}+10$ & $1.07 \mathrm{E}-01$ & $9.42 \mathrm{E}-02$ & $2.68 \mathrm{E}-02$ \\
\hline & 161 & 76.300 & $1.34 \mathrm{E}+11$ & $1.34 \mathrm{E}+11$ & 8.16E-01 & $8.21 \mathrm{E}-01$ & $2.05 \mathrm{E}-01$ \\
\hline \multirow[t]{7}{*}{93} & 124 & 85.879 & $1.61 \mathrm{E}+06$ & $1.86 \mathrm{E}+06$ & 8.88E-06 & $1.03 \mathrm{E}-05$ & $2.51 \mathrm{E}-06$ \\
\hline & 125 & 85.691 & $1.33 \mathrm{E}+07$ & $1.43 \mathrm{E}+07$ & 4.72E-05 & $5.07 \mathrm{E}-05$ & $1.33 \mathrm{E}-05$ \\
\hline & 141 & 77.834 & $4.19 \mathrm{E}+08$ & $3.27 \mathrm{E}+08$ & $1.90 \mathrm{E}-03$ & $1.49 \mathrm{E}-03$ & 4.87E-04 \\
\hline & 149 & 76.918 & $1.22 \mathrm{E}+10$ & $2.21 \mathrm{E}+10$ & $1.08 \mathrm{E}-02$ & $1.96 \mathrm{E}-02$ & $2.73 \mathrm{E}-03$ \\
\hline & 153 & 76.745 & $9.17 \mathrm{E}+11$ & $9.14 \mathrm{E}+11$ & $4.05 \mathrm{E}+00$ & $4.03 \mathrm{E}+00$ & $1.02 \mathrm{E}+00$ \\
\hline & 168 & 18.053 & $1.48 \mathrm{E}+08$ & $1.95 \mathrm{E}+08$ & 2.86E-05 & 3.77E-05 & $1.70 \mathrm{E}-06$ \\
\hline & 180 & 17.310 & $1.81 \mathrm{E}+07$ & $1.61 \mathrm{E}+07$ & 4.06E-06 & $3.62 \mathrm{E}-06$ & $2.32 \mathrm{E}-07$ \\
\hline \multirow[t]{8}{*}{94} & 102 & 134.057 & $5.65 \mathrm{E}+07$ & $7.05 \mathrm{E}+07$ & $2.02 \mathrm{E}-04$ & $2.52 \mathrm{E}-04$ & $5.70 \mathrm{E}-04$ \\
\hline & 129 & 85.412 & $1.12 \mathrm{E}+08$ & $1.03 \mathrm{E}+08$ & 8.56E-04 & 7.91E-04 & $2.41 \mathrm{E}-04$ \\
\hline & 131 & 84.933 & $3.98 \mathrm{E}+08$ & $4.08 \mathrm{E}+08$ & $3.01 \mathrm{E}-03$ & $3.09 \mathrm{E}-03$ & $8.43 \mathrm{E}-04$ \\
\hline & 135 & 80.977 & $1.22 \mathrm{E}+08$ & $1.52 \mathrm{E}+08$ & $9.59 \mathrm{E}-05$ & $1.20 \mathrm{E}-04$ & 4.49E-04 \\
\hline & 146 & 77.318 & $9.39 \mathrm{E}+08$ & $7.62 \mathrm{E}+08$ & 5.89E-03 & $4.78 \mathrm{E}-03$ & $1.50 \mathrm{E}-03$ \\
\hline & 157 & 76.635 & $1.90 \mathrm{E}+10$ & $1.88 \mathrm{E}+10$ & 1.17E-01 & $1.16 \mathrm{E}-01$ & $2.95 \mathrm{E}-02$ \\
\hline & 159 & 76.496 & $9.13 \mathrm{E}+10$ & $9.12 \mathrm{E}+10$ & 5.61E-01 & $5.60 \mathrm{E}-01$ & $1.41 \mathrm{E}-01$ \\
\hline & 161 & 76.449 & $9.54 \mathrm{E}+11$ & $9.46 \mathrm{E}+11$ & $5.85 \mathrm{E}+00$ & $5.80 \mathrm{E}+00$ & $1.47 \mathrm{E}+00$ \\
\hline \multirow[t]{3}{*}{95} & 113 & 127.904 & $8.38 \mathrm{E}+05$ & $9.63 \mathrm{E}+05$ & $1.44 \mathrm{E}-05$ & $1.65 \mathrm{E}-05$ & $6.06 \mathrm{E}-06$ \\
\hline & 127 & 85.711 & $4.13 \mathrm{E}+09$ & 4.27E+09 & $6.41 \mathrm{E}-03$ & $6.63 \mathrm{E}-03$ & $2.35 \mathrm{E}-02$ \\
\hline & 128 & 85.675 & $1.17 \mathrm{E}+09$ & $1.09 \mathrm{E}+09$ & 8.98E-03 & 8.39E-03 & $2.53 \mathrm{E}-03$ \\
\hline
\end{tabular}


Table 6. Cont.

\begin{tabular}{|c|c|c|c|c|c|c|c|}
\hline LL. & UL. & $\lambda$ & $A_{L}$ & $A_{V}$ & $f_{L}$ & $f_{V}$ & $S_{L}$ \\
\hline & 130 & 85.508 & $1.13 \mathrm{E}+09$ & $1.05 \mathrm{E}+09$ & 8.67E-03 & $8.09 \mathrm{E}-03$ & $2.44 \mathrm{E}-03$ \\
\hline & 136 & 79.422 & $9.86 \mathrm{E}+07$ & $1.21 \mathrm{E}+08$ & $1.22 \mathrm{E}-04$ & $1.50 \mathrm{E}-04$ & $5.74 \mathrm{E}-04$ \\
\hline & 140 & 78.617 & $3.20 \mathrm{E}+09$ & $3.87 \mathrm{E}+09$ & $3.83 \mathrm{E}-03$ & 4.64E-03 & $1.79 \mathrm{E}-02$ \\
\hline & 147 & 77.300 & $7.81 \mathrm{E}+10$ & $7.95 \mathrm{E}+10$ & $8.90 \mathrm{E}-02$ & $9.06 \mathrm{E}-02$ & $3.56 \mathrm{E}-01$ \\
\hline & 150 & 77.070 & $2.78 \mathrm{E}+10$ & $2.70 \mathrm{E}+10$ & $1.73 \mathrm{E}-01$ & $1.68 \mathrm{E}-01$ & $4.40 \mathrm{E}-02$ \\
\hline & 151 & 76.977 & $5.17 \mathrm{E}+11$ & $5.22 \mathrm{E}+11$ & $5.82 \mathrm{E}-01$ & $5.87 \mathrm{E}-01$ & $2.32 \mathrm{E}+00$ \\
\hline & 154 & 76.912 & $3.51 \mathrm{E}+09$ & $3.47 \mathrm{E}+09$ & $9.23 \mathrm{E}-03$ & $9.12 \mathrm{E}-03$ & $2.34 \mathrm{E}-03$ \\
\hline & 156 & 76.865 & $6.17 \mathrm{E}+10$ & $6.05 \mathrm{E}+10$ & $3.83 \mathrm{E}-01$ & $3.75 \mathrm{E}-01$ & $9.68 \mathrm{E}-02$ \\
\hline & 171 & 17.665 & $1.82 \mathrm{E}+09$ & $2.19 \mathrm{E}+09$ & $2.48 \mathrm{E}-05$ & $2.97 \mathrm{E}-05$ & $5.11 \mathrm{E}-04$ \\
\hline & 174 & 17.538 & $1.89 \mathrm{E}+08$ & $1.55 \mathrm{E}+08$ & $6.10 \mathrm{E}-05$ & $5.02 \mathrm{E}-05$ & $3.52 \mathrm{E}-06$ \\
\hline \multirow[t]{7}{*}{99} & 103 & 4170.843 & $1.27 \mathrm{E}+07$ & $1.66 \mathrm{E}+07$ & $2.27 \mathrm{E}+00$ & $2.97 \mathrm{E}+00$ & $2.16 \mathrm{E}-01$ \\
\hline & 104 & 3791.567 & $4.28 \mathrm{E}+07$ & $4.43 \mathrm{E}+07$ & $1.95 \mathrm{E}-01$ & $2.02 \mathrm{E}-01$ & $2.01 \mathrm{E}+00$ \\
\hline & 120 & 246.660 & $1.04 \mathrm{E}+08$ & $1.32 \mathrm{E}+08$ & $3.86 \mathrm{E}-03$ & $4.89 \mathrm{E}-03$ & $6.03 \mathrm{E}-03$ \\
\hline & 127 & 230.901 & $2.12 \mathrm{E}+07$ & $1.99 \mathrm{E}+07$ & $6.43 \mathrm{E}-04$ & $6.04 \mathrm{E}-04$ & 7.94E-04 \\
\hline & 136 & 190.307 & $3.26 \mathrm{E}+09$ & $3.78 \mathrm{E}+09$ & $5.55 \mathrm{E}-02$ & $6.42 \mathrm{E}-02$ & $1.03 \mathrm{E}-01$ \\
\hline & 140 & 185.748 & $1.50 \mathrm{E}+07$ & $5.37 \mathrm{E}+06$ & 2.37E-04 & $8.49 \mathrm{E}-05$ & $1.39 \mathrm{E}-04$ \\
\hline & 147 & 178.561 & $8.45 \mathrm{E}+07$ & $7.89 \mathrm{E}+07$ & 1.19E-03 & $1.11 \mathrm{E}-03$ & $1.89 \mathrm{E}-03$ \\
\hline \multirow[t]{4}{*}{100} & 118 & 257.851 & $4.74 \mathrm{E}+08$ & $5.50 \mathrm{E}+08$ & $1.42 \mathrm{E}-02$ & $1.64 \mathrm{E}-02$ & $1.20 \mathrm{E}-02$ \\
\hline & 137 & 192.241 & $1.59 \mathrm{E}+09$ & $1.88 \mathrm{E}+09$ & 2.64E-02 & $3.13 \mathrm{E}-02$ & $1.67 \mathrm{E}-02$ \\
\hline & 138 & 189.973 & $7.96 \mathrm{E}+08$ & $1.05 \mathrm{E}+09$ & $1.29 \mathrm{E}-02$ & $1.70 \mathrm{E}-02$ & 8.08E-03 \\
\hline & 154 & 178.253 & $8.62 \mathrm{E}+07$ & $7.01 \mathrm{E}+07$ & $1.23 \mathrm{E}-03$ & $1.00 \mathrm{E}-03$ & 7.23E-04 \\
\hline \multirow[t]{5}{*}{101} & 103 & 6293.616 & $1.73 \mathrm{E}+06$ & $1.61 \mathrm{E}+06$ & $5.11 \mathrm{E}-02$ & $4.75 \mathrm{E}-02$ & $1.06 \mathrm{E}+00$ \\
\hline & 104 & 5461.860 & $1.03 \mathrm{E}+08$ & $8.81 \mathrm{E}+07$ & $4.81 \mathrm{E}-01$ & $4.13 \mathrm{E}-01$ & $3.96 \mathrm{E}+00$ \\
\hline & 121 & 250.423 & $2.85 \mathrm{E}+08$ & $2.28 \mathrm{E}+08$ & $4.79 \mathrm{E}-03$ & $3.83 \mathrm{E}-03$ & $3.05 \mathrm{E}-03$ \\
\hline & 136 & 193.272 & $1.93 \mathrm{E}+09$ & $1.60 \mathrm{E}+09$ & $5.14 \mathrm{E}-02$ & $4.27 \mathrm{E}-02$ & $3.19 \mathrm{E}-02$ \\
\hline & 176 & 20.080 & $2.14 \mathrm{E}+08$ & $2.43 \mathrm{E}+08$ & $3.88 \mathrm{E}-05$ & 4.40E-05 & $2.56 \mathrm{E}-06$ \\
\hline \multirow[t]{4}{*}{102} & 104 & 6107.597 & $1.76 \mathrm{E}+07$ & $1.79 \mathrm{E}+07$ & $4.57 \mathrm{E}-02$ & $4.65 \mathrm{E}-02$ & $3.59 \mathrm{E}-01$ \\
\hline & 120 & 252.893 & $3.97 \mathrm{E}+08$ & $4.73 E+08$ & $1.58 \mathrm{E}-02$ & $1.89 \mathrm{E}-02$ & $2.27 \mathrm{E}-02$ \\
\hline & 151 & 180.029 & $2.13 \mathrm{E}+08$ & $2.03 E+08$ & $3.07 \mathrm{E}-03$ & $2.93 \mathrm{E}-03$ & $4.94 \mathrm{E}-03$ \\
\hline & 154 & 179.672 & $1.08 \mathrm{E}+07$ & $9.39 \mathrm{E}+06$ & $1.36 \mathrm{E}-04$ & $1.18 \mathrm{E}-04$ & $8.06 \mathrm{E}-05$ \\
\hline \multirow[t]{5}{*}{103} & 129 & 243.394 & $3.27 \mathrm{E}+08$ & $2.77 \mathrm{E}+08$ & $2.03 \mathrm{E}-02$ & $1.72 \mathrm{E}-02$ & $1.63 \mathrm{E}-02$ \\
\hline & 131 & 239.543 & $4.06 \mathrm{E}+06$ & $3.45 \mathrm{E}+06$ & $2.44 \mathrm{E}-04$ & $2.08 \mathrm{E}-04$ & $1.93 \mathrm{E}-04$ \\
\hline & 157 & 183.504 & $5.74 \mathrm{E}+06$ & $4.66 \mathrm{E}+06$ & 2.03E-04 & $1.65 \mathrm{E}-04$ & $1.23 \mathrm{E}-04$ \\
\hline & 159 & 182.707 & $8.27 \mathrm{E}+07$ & $1.09 \mathrm{E}+08$ & $2.90 \mathrm{E}-03$ & $3.80 \mathrm{E}-03$ & $1.74 \mathrm{E}-03$ \\
\hline & 161 & 182.440 & $4.32 \mathrm{E}+07$ & $4.48 \mathrm{E}+07$ & $1.51 \mathrm{E}-03$ & $1.56 \mathrm{E}-03$ & $9.07 \mathrm{E}-04$ \\
\hline \multirow[t]{2}{*}{104} & 116 & 291.728 & $9.43 \mathrm{E}+08$ & $1.14 \mathrm{E}+09$ & $3.19 \mathrm{E}-02$ & $3.86 \mathrm{E}-02$ & $2.62 \mathrm{E}-02$ \\
\hline & 117 & 287.001 & $1.13 \mathrm{E}+09$ & $1.71 \mathrm{E}+09$ & $1.02 \mathrm{E}-02$ & $1.55 \mathrm{E}-02$ & $8.26 \mathrm{E}-03$ \\
\hline
\end{tabular}


Table 6. Cont.

\begin{tabular}{|c|c|c|c|c|c|c|c|}
\hline LL. & UL. & $\lambda$ & $A_{L}$ & $A_{V}$ & $f_{L}$ & $f_{V}$ & $S_{L}$ \\
\hline & 134 & 212.995 & $1.14 \mathrm{E}+09$ & $1.22 \mathrm{E}+09$ & $1.95 \mathrm{E}-02$ & 2.09E-02 & $1.21 \mathrm{E}-02$ \\
\hline \multirow[t]{3}{*}{105} & 116 & 299.399 & $3.81 \mathrm{E}+07$ & $3.10 \mathrm{E}+07$ & $1.51 \mathrm{E}-03$ & $1.23 \mathrm{E}-03$ & $1.25 \mathrm{E}-03$ \\
\hline & 129 & 250.183 & $3.25 \mathrm{E}+08$ & $3.71 \mathrm{E}+08$ & $2.13 \mathrm{E}-02$ & $2.44 \mathrm{E}-02$ & $1.76 \mathrm{E}-02$ \\
\hline & 135 & 215.595 & $1.17 \mathrm{E}+08$ & $9.91 \mathrm{E}+07$ & $1.73 \mathrm{E}-03$ & $1.47 \mathrm{E}-03$ & $2.07 \mathrm{E}-03$ \\
\hline \multirow[t]{5}{*}{106} & 122 & 255.082 & $6.90 \mathrm{E}+08$ & $6.61 \mathrm{E}+08$ & $3.36 \mathrm{E}-02$ & $3.22 \mathrm{E}-02$ & $2.82 \mathrm{E}-02$ \\
\hline & 124 & 249.236 & $1.84 \mathrm{E}+05$ & $1.86 \mathrm{E}+05$ & 8.57E-06 & 8.68E-06 & 7.03E-06 \\
\hline & 141 & 191.725 & $1.24 \mathrm{E}+09$ & $1.12 \mathrm{E}+09$ & $3.41 \mathrm{E}-02$ & $3.10 \mathrm{E}-02$ & $2.15 \mathrm{E}-02$ \\
\hline & 144 & 188.967 & $8.21 \mathrm{E}+08$ & $6.17 \mathrm{E}+08$ & $2.20 \mathrm{E}-02$ & $1.65 \mathrm{E}-02$ & $1.37 \mathrm{E}-02$ \\
\hline & 153 & 185.251 & $1.70 \mathrm{E}+07$ & $1.60 \mathrm{E}+07$ & 4.37E-04 & 4.12E-04 & $2.66 \mathrm{E}-04$ \\
\hline 107 & 179 & 19.870 & $3.23 \mathrm{E}+07$ & $3.64 \mathrm{E}+07$ & $1.34 \mathrm{E}-05$ & $1.51 \mathrm{E}-05$ & $8.75 \mathrm{E}-07$ \\
\hline \multirow[t]{4}{*}{110} & 136 & 205.651 & $6.19 \mathrm{E}+06$ & $7.54 \mathrm{E}+06$ & $1.96 \mathrm{E}-04$ & $2.39 \mathrm{E}-04$ & $1.33 \mathrm{E}-04$ \\
\hline & 139 & 201.864 & $3.20 \mathrm{E}+05$ & $3.20 \mathrm{E}+05$ & $2.49 \mathrm{E}-04$ & $1.37 \mathrm{E}-05$ & $7.06 \mathrm{E}-04$ \\
\hline & 140 & 200.337 & $7.48 \mathrm{E}+06$ & $6.53 \mathrm{E}+06$ & $2.25 \mathrm{E}-04$ & $1.97 \mathrm{E}-04$ & $1.48 \mathrm{E}-04$ \\
\hline & 172 & 20.350 & $4.69 \mathrm{E}+09$ & $3.62 \mathrm{E}+09$ & 8.73E-04 & $6.74 \mathrm{E}-04$ & $5.85 \mathrm{E}-05$ \\
\hline 111 & 128 & 256.624 & $5.49 \mathrm{E}+08$ & $6.80 \mathrm{E}+08$ & $3.80 \mathrm{E}-02$ & $4.70 \mathrm{E}-02$ & $3.21 \mathrm{E}-02$ \\
\hline \multirow[t]{3}{*}{112} & 128 & 259.202 & $2.18 \mathrm{E}+08$ & $2.03 \mathrm{E}+08$ & $1.54 \mathrm{E}-02$ & $1.43 \mathrm{E}-02$ & $1.31 \mathrm{E}-02$ \\
\hline & 130 & 257.677 & $3.12 \mathrm{E}+08$ & $3.15 E+08$ & $2.17 \mathrm{E}-02$ & $2.19 \mathrm{E}-02$ & $1.84 \mathrm{E}-02$ \\
\hline & 139 & 205.896 & $1.37 \mathrm{E}+06$ & $1.16 \mathrm{E}+06$ & $6.09 \mathrm{E}-05$ & $5.18 \mathrm{E}-05$ & $4.13 \mathrm{E}-05$ \\
\hline \multirow[t]{5}{*}{116} & 118 & 4132.808 & $1.35 \mathrm{E}+07$ & $1.44 \mathrm{E}+07$ & $1.10 \mathrm{E}-01$ & $1.17 \mathrm{E}-01$ & $1.50 \mathrm{E}+00$ \\
\hline & 120 & 2755.630 & $7.41 \mathrm{E}+07$ & $7.92 \mathrm{E}+07$ & $3.82 \mathrm{E}+00$ & $4.09 \mathrm{E}+00$ & $4.51 \mathrm{E}-01$ \\
\hline & 121 & 2615.280 & $2.25 \mathrm{E}+07$ & $2.85 \mathrm{E}+07$ & $8.78 \mathrm{E}-02$ & $1.11 \mathrm{E}-01$ & $7.56 \mathrm{E}-01$ \\
\hline & 147 & 523.819 & $1.12 \mathrm{E}+06$ & $1.36 \mathrm{E}+06$ & $3.96 \mathrm{E}-04$ & $4.83 \mathrm{E}-04$ & $2.80 \mathrm{E}-04$ \\
\hline & 172 & 21.814 & $1.64 \mathrm{E}+08$ & $1.57 \mathrm{E}+08$ & $3.37 \mathrm{E}-05$ & $3.23 \mathrm{E}-05$ & $2.42 \mathrm{E}-06$ \\
\hline 117 & 154 & 521.441 & $4.10 \mathrm{E}+06$ & $5.56 \mathrm{E}+06$ & $5.01 \mathrm{E}-04$ & $6.80 \mathrm{E}-04$ & $8.60 \mathrm{E}-04$ \\
\hline \multirow[t]{2}{*}{118} & 122 & 3996.219 & $2.30 \mathrm{E}+07$ & $1.82 \mathrm{E}+07$ & $2.76 \mathrm{E}-01$ & $2.18 \mathrm{E}-01$ & $3.63 \mathrm{E}+00$ \\
\hline & 124 & 2922.453 & $3.13 \mathrm{E}+02$ & $2.78 \mathrm{E}+02$ & $2.00 \mathrm{E}-06$ & $1.78 \mathrm{E}-06$ & $1.93 \mathrm{E}-05$ \\
\hline 120 & 179 & 21.447 & $1.40 \mathrm{E}+07$ & $1.17 \mathrm{E}+07$ & $6.76 \mathrm{E}-06$ & $5.65 \mathrm{E}-06$ & 4.77E-07 \\
\hline \multirow[t]{2}{*}{125} & 154 & 733.063 & $3.15 \mathrm{E}+05$ & $3.83 \mathrm{E}+05$ & $9.25 \mathrm{E}-05$ & $1.12 \mathrm{E}-04$ & $2.23 \mathrm{E}-04$ \\
\hline & 172 & 22.108 & $1.39 \mathrm{E}+08$ & $1.30 \mathrm{E}+08$ & $2.85 \mathrm{E}-05$ & 2.67E-05 & $2.08 \mathrm{E}-06$ \\
\hline 127 & 134 & 1593.628 & $3.26 \mathrm{E}+07$ & $3.28 \mathrm{E}+07$ & $2.80 \mathrm{E}-01$ & $2.81 \mathrm{E}-01$ & $3.11 \mathrm{E}+00$ \\
\hline 131 & 139 & 1073.552 & $1.17 \mathrm{E}+03$ & $1.02 \mathrm{E}+03$ & $1.41 \mathrm{E}-06$ & $1.24 \mathrm{E}-06$ & $4.98 \mathrm{E}-06$ \\
\hline \multirow[t]{4}{*}{134} & 136 & 3378.317 & $2.26 \mathrm{E}+07$ & $2.20 \mathrm{E}+07$ & $1.38 \mathrm{E}-01$ & $1.34 \mathrm{E}-01$ & $1.69 \mathrm{E}+00$ \\
\hline & 138 & 2773.696 & $1.53 \mathrm{E}+07$ & $1.26 \mathrm{E}+07$ & $5.22 \mathrm{E}-02$ & $4.30 \mathrm{E}-02$ & $5.21 \mathrm{E}-01$ \\
\hline & 140 & 2354.678 & $3.49 \mathrm{E}+07$ & $3.23 \mathrm{E}+07$ & $1.45 \mathrm{E}-01$ & $1.34 \mathrm{E}-01$ & $1.12 \mathrm{E}+00$ \\
\hline & 156 & 1377.586 & $1.24 \mathrm{E}+06$ & $1.63 \mathrm{E}+06$ & $3.10 \mathrm{E}-02$ & $3.25 \mathrm{E}-03$ & 4.97E-01 \\
\hline
\end{tabular}


Table 6. Cont.

\begin{tabular}{cccccccc}
\hline LL. & UL. & $\lambda$ & $A_{L}$ & $A_{V}$ & $f_{L}$ & $f_{V}$ & $S_{L}$ \\
\hline & 172 & 22.450 & $7.31 \mathrm{E}+07$ & $7.29 \mathrm{E}+07$ & $1.65 \mathrm{E}-05$ & $1.65 \mathrm{E}-05$ & $1.22 \mathrm{E}-06$ \\
\hline 135 & 140 & 2537.653 & $5.86 \mathrm{E}+07$ & $7.69 \mathrm{E}+07$ & $2.36 \mathrm{E}+00$ & $3.10 \mathrm{E}+00$ & $3.71 \mathrm{E}-01$ \\
& 151 & 1503.726 & $1.23 \mathrm{E}+04$ & $1.36 \mathrm{E}+04$ & $1.03 \mathrm{E}-04$ & $1.14 \mathrm{E}-04$ & $2.31 \mathrm{E}-05$ \\
\hline \multirow{2}{*}{137} & 143 & 3528.800 & $7.96 \mathrm{E}+06$ & $8.68 \mathrm{E}+06$ & $4.86 \mathrm{E}-02$ & $5.30 \mathrm{E}-02$ & $5.65 \mathrm{E}-01$ \\
& 168 & 23.382 & $1.24 \mathrm{E}+08$ & $1.02 \mathrm{E}+08$ & $2.51 \mathrm{E}-05$ & $2.06 \mathrm{E}-05$ & $1.93 \mathrm{E}-06$ \\
138 & 141 & 6247.764 & $4.59 \mathrm{E}+05$ & $3.86 \mathrm{E}+05$ & $1.34 \mathrm{E}-02$ & $1.13 \mathrm{E}-02$ & $2.76 \mathrm{E}-01$ \\
& 143 & 4518.690 & $8.43 \mathrm{E}+06$ & $9.93 \mathrm{E}+06$ & $9.12 \mathrm{E}-02$ & $1.07 \mathrm{E}-01$ & $1.36 \mathrm{E}+00$ \\
\hline 140 & 143 & 6363.023 & $2.19 \mathrm{E}+05$ & $2.22 \mathrm{E}+05$ & $8.34 \mathrm{E}-02$ & $8.48 \mathrm{E}-02$ & $4.05 \mathrm{E}-03$ \\
& 152 & 3636.691 & $2.35 \mathrm{E}+05$ & $1.37 \mathrm{E}+06$ & $1.67 \mathrm{E}-02$ & $9.72 \mathrm{E}-02$ & $8.12 \mathrm{E}-03$ \\
\hline 152 & 176 & 22.613 & $4.36 \mathrm{E}+07$ & $5.72 \mathrm{E}+07$ & $1.31 \mathrm{E}-05$ & $1.72 \mathrm{E}-05$ & $9.79 \mathrm{E}-07$ \\
\hline 153 & 176 & 22.615 & $5.83 \mathrm{E}+08$ & $5.05 \mathrm{E}+08$ & $1.33 \mathrm{E}-04$ & $1.16 \mathrm{E}-04$ & $9.91 \mathrm{E}-06$ \\
\hline 154 & 178 & 22.353 & $6.09 \mathrm{E}+06$ & $6.74 \mathrm{E}+06$ & $1.79 \mathrm{E}-03$ & $1.98 \mathrm{E}-03$ & $4.77 \mathrm{E}-03$ \\
\hline 156 & 178 & 22.362 & $5.32 \mathrm{E}+04$ & $6.07 \mathrm{E}+04$ & $2.00 \mathrm{E}-08$ & $2.51 \mathrm{E}-08$ & $1.47 \mathrm{E}-09$ \\
\hline 167 & 172 & 655.182 & $2.54 \mathrm{E}+08$ & $2.09 \mathrm{E}+08$ & $4.89 \mathrm{E}-02$ & $4.04 \mathrm{E}-02$ & $1.06 \mathrm{E}-01$ \\
& 176 & 533.321 & $7.51 \mathrm{E}+08$ & $6.83 \mathrm{E}+08$ & $9.61 \mathrm{E}-02$ & $8.74 \mathrm{E}-02$ & $1.69 \mathrm{E}-01$ \\
\hline 168 & 172 & 663.050 & $1.66 \mathrm{E}+08$ & $1.55 \mathrm{E}+08$ & $3.06 \mathrm{E}-02$ & $2.86 \mathrm{E}-02$ & $6.67 \mathrm{E}-02$ \\
& 176 & 538.524 & $4.44 \mathrm{E}+08$ & $4.80 \mathrm{E}+08$ & $6.26 \mathrm{E}-02$ & $6.77 \mathrm{E}-02$ & $1.11 \mathrm{E}-01$ \\
\hline 172 & 180 & 823.268 & $7.29 \mathrm{E}+07$ & $8.43 \mathrm{E}+07$ & $7.22 \mathrm{E}-02$ & $8.35 \mathrm{E}-02$ & $2.73 \mathrm{E}-01$ \\
\hline 174 & 179 & 1013.347 & $3.90 \mathrm{E}+07$ & $3.27 \mathrm{E}+07$ & $4.20 \mathrm{E}-02$ & $3.53 \mathrm{E}-02$ & $1.40 \mathrm{E}-01$ \\
\hline 176 & 180 & 1154.415 & $4.28 \mathrm{E}+04$ & $4.76 \mathrm{E}+04$ & $1.18 \mathrm{E}-04$ & $1.31 \mathrm{E}-04$ & $7.48 \mathrm{E}-04$ \\
\hline
\end{tabular}

\section{Conclusions}

In the present study, fine structure energy levels, oscillator strengths, line strengths, transition probabilities and wavelengths for transitions among levels belonging to Be-like iron are presented. We have used extensive CI wave functions based on large CSF expansions to produce 182 LSJ-coupling levels with various $J$-values. The self-consistent field approximation and the Breit interaction Hamiltonian as well as QED effects have been included in the calculations to improve the generated wave functions. The calculated energy levels and weighted oscillator strengths show a good agreement with both theoretical and experimental data from the literature. In addition, we have obtained some new and previously unpublished energy levels for this ion. Our results are useful for many applications such as controlled thermonuclear fusion, laser and plasma physics as well as astrophysics. 


\section{Author Contributions}

Ahmed Abou El-Maaref suggested the main idea of the paper, performed the calculations, and wrote the great part of the manuscript. Alfred Müller and Stefan Schippers were involved in the discussion, particularly, of the astrophysical data needs, provided the required computer hardware, searched the literature and wrote parts of the manuscript.

\section{Conflicts of Interest}

The authors declare no conflict of interest.

\section{References}

1. Brown, G.V.; Beiersdorfer, P.; Liedahl, D.A.; Widmann, K.; Kahn, S.M.; Clothiaux E.J. Laboratory measurements and identification of the Fe XVIII-XXIV L-shell x-ray line emission. Astrophys. J. Suppl. Ser. 2002, 140, 589-607.

2. Chen, H.; Gu, M.F.; Behar, E.; Brown, G.V.; Kahn, S.M.; Beiersdorfer, P. Laboratory measurements of high-n iron L-shell x-ray lines. Astrophys. J. Suppl. Ser. 2007, 168, 319-336.

3. Doschek, G.A.; Feldman, U. The solar UV-x-ray spectrum from 1.5 to $2000 \AA$ A. J. Phys. B 2010, 43, 232001:1-232001:23.

4. Raymond, J.C. Charge transfer x-rays in solar and stellar flares. Astron. Nachr. 2012, 333, 305-308.

5. Morita, S.; Dong, C.F.; Kobayashi, M.; Goto, M.; Huang, X.L.; Murakami, I.; Oishi, T.; Wang, E.H.; Ashikawa, N.; Fujii, K.; et al. Effective screening of iron impurities in the ergodic layer of the Large Helical Device with a metallic first wall. Nucl. Fusion 2013, 53, 093017:1-093017:12.

6. Boiko, V.A.; Faenov, A.I.; Pikuz, S.A. X-ray spectroscopy of multiply-charged ions from laser plasmas. J. Quant. Spectrosc. Radiat. Transf. 1978, 19, 11-50.

7. Asplund, M.; Grevesse, N.; Sauval, A.J.; Scott, P. The chemical composition of the sun. Annu. Rev. Astron. Astrophys. 2009, 47, 481-522.

8. Cheng, K.T.; Kim, Y.K.; Desclaux, J.P. Electric dipole, quadrupole, and magnetic dipole transition probabilities of ions isoelectronic to the first-row atoms, Li through F. At. Data Nucl. Data Tables 1979, 24, 111-189.

9. Corliss, C.; Sugar, J. Energy levels of iron, Fe I through Fe XXVI. J. Phys. Chem. Ref. Data 1998, 11, 135-241.

10. Bhatia, A.K.; Mason H.E. Atomic calculation for Fe XXIII, UV, and X-ray lines. Astron. Astrophys. 1981, 103, 324-330.

11. Glass, R. Magnetic dipole transitions in the beryllium isoelectronic sequence. Astrophys. Space Sci. 1983, 91, 417-426.

12. Idrees, M.; Das, B.P. Multiconfiguration Dirac-Fock calculation of the forbidden M1 transitions in the beryllium isoelectronic sequence. J. Phys. B 1989, 22, 3609-3613. 
13. Tully, J.A.; Seaton, M.J.; Berrington, K.A. Atomic data for opacity calculations. XIV. The beryllium sequence. J. Phys. B 1990, 23, 3811-3837.

14. Eissner, W.B.; Tully, J.A. Spectral lines in the beryllium sequence. Astron. Astrophys. 1992, 253, 625-631.

15. Fritzsche, S.; Grant, I.P. Ab-initio calculation of the $2 s^{2}{ }^{1} S_{o}-2 s 3 p{ }^{3} P_{1}$ intercombination transition in beryllium-like ions. Phys. Scr. 1994, 50, 473-480.

16. Safronova, U.I.; Shlyaptseva A.S.; Kato, T.; Masai, K.; Vainshtein, L.A. Cross sections and rate coeffiecients for excitation of $\Delta n=0$ transitions in Be-like ions $6 \geq Z \leq 56$. At. Data Nucl. Data Tables 1995, 60, 1-36.

17. Chen, G.-X.; Ong, P.P. Relativistic calculations for Fe XXIII: Atomic structure. Phys. Rev. A 1998, $58,1070-1081$.

18. Zhang, H.L.; Pradhan, A.K. Relativistic excitation rate coefficients for Fe XXII with inclusion of radiation damping. Astron. Astrophys. Suppl. Ser. 1997, 123, 575-580.

19. Safronova, U.I.; Johnson, W.; Safronova, M.S.; Derevianko, A. Relativistic many-body calculations of transition probabilities for the $2 l_{1} 2 l_{2}[L S J]-2 l_{3} 2 l_{4}\left[L^{\prime} S^{\prime} J^{\prime}\right]$ lines in Be-like ions. Phys. Scr. 1999, 59, 286-295.

20. Zhang, H.L.; Fontes, C.J. Relativistic distorted-wave collision strengths for the $16 \Delta n=0$ optically allowed transitions with $\Delta n=2$ in the 67 Be-like ions with $26 \leq Z \leq 92$. At. Data Nucl. Data Tables 2013, 99, 416-430.

21. Kingston, A.E.; Hibbert, A. The calculation of the line strengths for magnetic dipole transitions between the $2 s^{2}, 2 s 2 p$ and $2 p^{2}$ states in Be-like ions. Phys. Scr. 2001, 64, 58-62.

22. Moribayashi, K.; Kato, T. Dielectronic recombination to the excited states of Be-like Fe ions. Phys. Scr. 1997, 55, 286-297.

23. Cowan, R.D. The Theory of Atomic Structure and Spectra; University of California Press: Berkeley, CA, USA, 1981.

24. Chidichimo, M.C.; del Zanna, G.; Mason, H.E.; Badnell, N.R.; Tully, J.A.; Berrington, K.A. Electron excitation of Be-like Fe XXIII for the $n=2,3,4$ configurations. Astron. Astrophys. 2005, 430, 331-341.

25. Chidichimo, M.C.; Zeman, V.; Tully, J.A.; Berrington, K.A. Atomic data from the IRON Project. XXXVI. Electron excitation of Be-like Fe XXIII between $1 s^{2} 2 l_{1} 2 l_{2} S L J$ and $1 s^{2} 2 l_{3} 2 l_{4} S^{\prime} L^{\prime} J^{\prime}$. Astron. Astrophys. Suppl. Ser. 1999, 137, 175-184.

26. Del Zanna, G.; Chidichimo, M.C.; Mason, H.E. Benchmarking atomic data for astrophysics: Fe XXIII. Astron. Astrophys. 2005, 432, 1137-1150.

27. Santos, J.P.; Marques, J.P.; Costa, A.M.; Martins, M.C.; Indelicato, P.; Parente, P. Transition probability values of the $1 s^{2} 2 s 3 p{ }^{3} P_{0}$ level in Be-like ions. Phys. Scr. 2013, T156, 014020: 1-014020:2.

28. Yang, J.-H.; Li, P.; Zhang, J.-P.; Li, H.-L. Relativistic calculations for Be-like iron. Commun. Theor. Phys. 2008, 50, 468-472.

29. Charro, E.; Martín, I.; Lavín, C. Multi-configuration Dirac-Fock and relativistic quantum defect orbital study of triplet-triplet transitions in beryllium-like ions. J. Quant. Spectrosc. Radiat. Transf. 1996, 56, 241-253. 
30. Charro, E.; Martin, I. Complementary investigations using the MCDF and RQDO methods. Int. J. Quantum Chem. 2005, 104, 446-457.

31. Landi, E.; Gu, M.F. Atomic data for high-energy configurations in Fe XVII-XXIII. Astrophys. J. 2006, 640, 1171-1179.

32. Grant, I.P. Relativistic Quantum Theory of Atoms and Molecules; Springer: New York, NY, USA, 2007.

33. Jönsson, P.; Gaigalas, G.; Bieroń, J.; Froese Fischer, C.; Grant, I.P. New version: Grasp2K relativistic atomic structure package. Comput. Phys. Commun. 2013, 184, 2197-2203.

34. NIST Atomic Spectra Database (Version 5): Available online: http://www.nist.gov/ pml/data/asd.cfm (accessed on 1 February 2014).

35. Fritzsche, S.; Dong, C.Z.; Gaigalas, G. Theoretical wavelengths and transition probabilites for the $3 d^{9}-3 d^{8} 4 p$ and $3 d^{8} 4 s-3 d^{8} 4 p$ transition arrays in Ni II. At. Data Nucl. Data Tables 2000, 76, $155-176$.

36. Rynkun, P.; Jönsson, P.; Gaigalas, G.; Froese Fischer, C. Energies and E1, M1, E2, and M2 transition rates for states of the $2 s^{2} 2 p^{3}, 2 s 2 p^{4}$, and $2 p^{5}$ configurations in nitrogen-like ions between F III and Kr XXX. At. Data Nucl. Data Tables 2014, 100, 315-402.

37. Nazé, C.; Gaidamauskas, E.; Gaigalas, G.; Godefroid, M.; Jönsson, P. ris3: A program for relativistic isotope shift calculations. Comput. Phys. Commun. 2013, 184, 2187-2196.

38. CHIANTI Atomic Database for Spectroscopic Diagnostics of Astrophysical Plasmas. Available online: http://www.chiantidatabase.org/dbase/fe/fe_23/fe_23.elvlc (accessed on 1 February 2014)

39. Gu, M.F. Wavelengths of $2 l-3 l^{\prime}$ transitions in L-shell ions of iron and nickel: A combined configuration interaction and many-body perturbation theory approach. Astrophys. J. Suppl. Ser. 2005, 156, 105-110.

40. Gillaspy, J. Trapping Highly Charged Ions: Fundamentals and Applications; Nova Publisher: New York, NY, USA, 2001.

41. Fuhr, J.R.; Martin, G.A.; Wiese, W.L. Atomic transition probabilities iron through nickel. J. Phys. Chem. Ref. Data 1988, 17, 1-499.

42. Nussbaumer, H.; Storey, P.J. Transition probabilities for Ca XVII, Fe XXIII, Kr XXXIII and Mo XXXIX. J. Phys. B 1979, 12, 1647-1652.

43. Fuhr, J.R.; Martin, G.A.; Wiese, W.L.; Younger, S.M. Atomic transition probabilities for iron, cobalt, and nickel (a critical data compilation of allowed lines). J. Phys. Chem. Ref. Data 1981, $10,305-565$.

44. Bromage, G.E.; Cowan, R.D.; Fawcett, B.C.; Ridgeley, A. Classification of Be I-like and B I-like iron and vanadium spectra from laser-produced plasmas. J. Opt. Soc. Am. 1978, 68, 48-51.

45. Fawcett, B.C.; Jordan, C.; Lemen, J.R.; Phillips, K.J.H. Rutherford Appleton Laboratory Report $R A L$; Rutherford Appleton Laboratory: Oxfordshire, UK, 1986; pp. 86-94.

46. Nussbaumer, H. Spectral lines in the Be I isoelectronic sequence. Astron. Astrophys. 1972, 16, 77-80.

47. Shirai, T.; Sugar J.; Musgrove, A.; Wiese, W.L. Spectral data for highly ionized atoms: Ti, V, Cr, $\mathrm{Mn}, \mathrm{Fe}, \mathrm{Co}, \mathrm{Ni}, \mathrm{Cu}, \mathrm{Kr}$, and Mo. J. Phys. Chem. Ref. Data 2000, Monograph No. 8, 1-636. 
48. Buchet, J.P.; Buchet-Poulizac M.C.; Denis, A.; Desesquelles, J.; Druett, M. Radiative lifetimes and oscillator strengths for $2 s-2 p$ transitions in He-, Li-, and Be-like iron. Phys. Rev. A 1984, 30, 309-315.

49. Tully, J.A.; Seaton, M.J.; Berrington, K.A. An update of oscillator strengths and photoionisation cross sections for the Be isoelectronic sequence. J. Phys. IV 1991, 1, C1:169-C1:178.

50. Cheng, K.T.; Chen, M.H. Hyperfine quenching of the $2 s 2 p^{3} P_{0}$ state of beryllium-like ions. Phys. Rev. A 2009, 77, 052504:1-052504:14.

51. Wyart, J.-F.; Fajardo, M.; Mißalla, T.; Chenais-Popovics, C.; Klopfel, D.; Förster, E. Observation and analysis of x-ray spectra of highly-ionized atoms produced by laser irradiation in the wavelength range $0.60 \mathrm{~nm}$ to $0.95 \mathrm{~nm}$. Phys. Scr. 1999, T83, 35-43.

(c) 2015 by the authors; licensee MDPI, Basel, Switzerland. This article is an open access article distributed under the terms and conditions of the Creative Commons Attribution license (http://creativecommons.org/licenses/by/4.0/). 